\title{
Spontaneous Emission of Near-Infrared Quantum Dots Controlled with Photonic Crystals
}

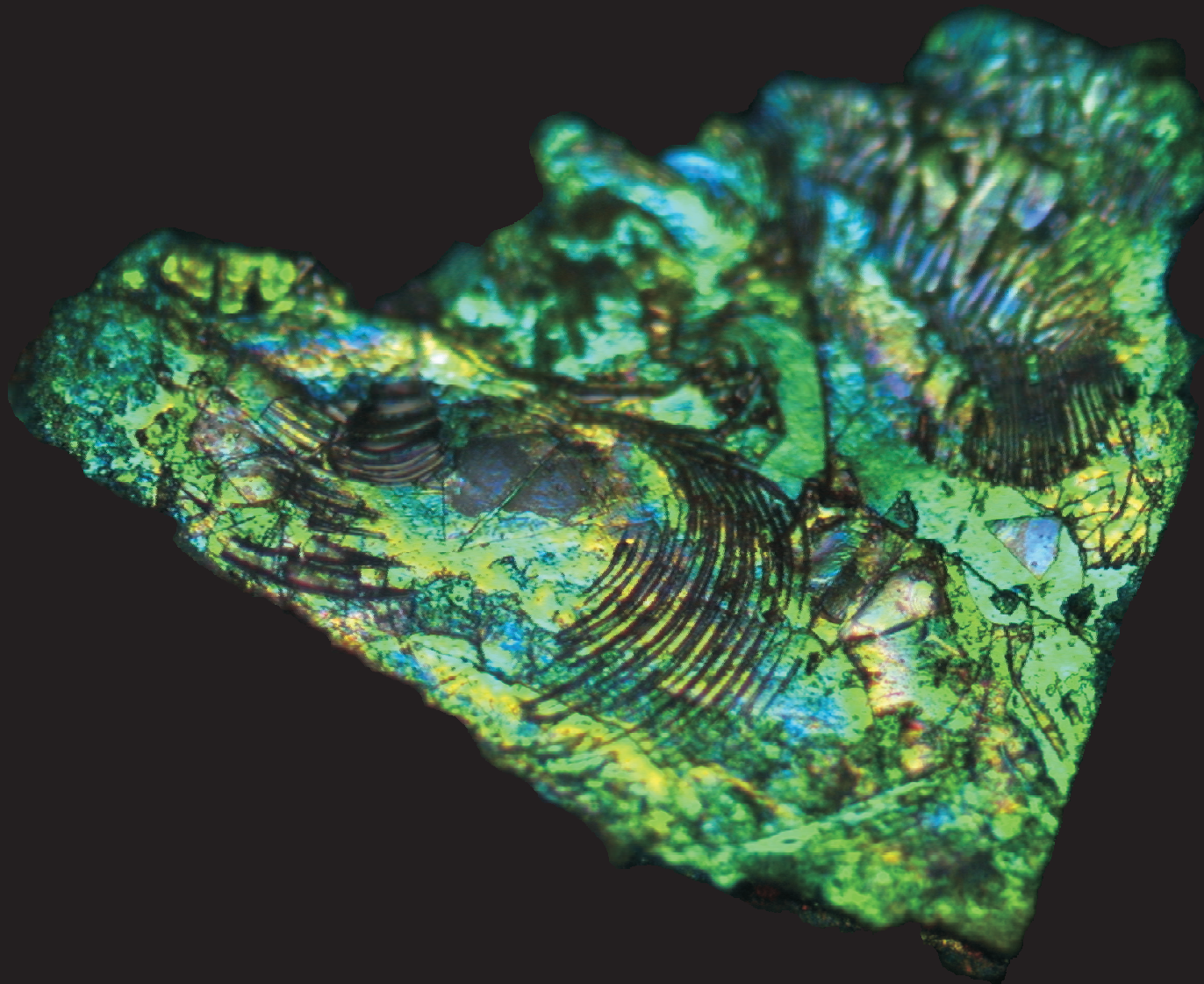

Bart H. Husken 
backside of cover (empty) 


\section{SPONTANEOUS EMISSION OF NEAR-INFRARED QUANTUM DOTS CONTROLLED WITH PHOTONIC CRYSTALS}


Promotiecommissie:

Promotor: $\quad$ prof. dr. W. L. Vos

Voorzitter: $\quad$ prof. dr. G. van der Steenhoven

Overige leden: $\quad$ prof. dr. M. Bayer

prof. dr. T. Gregorkiewicz

prof. dr. P. J. Kelly

dr. A.F. Koenderink

prof. dr. A. Lagendijk

Paranimfen: $\quad$ ir. D. A. Bekke

dr. ir. S. Gerritsen

The work described in this thesis is financially supported by the "Nederlandse Organisatie voor Wetenschappelijk Onderzoek" (NWO). This work was carried out in the group Photonic Bandgaps, at the Center for Nanophotonics, FOM Institute for Atomic and Molecular Physics (AMOLF), Amsterdam, The Netherlands, and in the group Complex Photonic Systems (COPS), MESA ${ }^{+}$Institute for Nanotechnology and Faculty of Science and Technology, University of Twente, Enschede, The Netherlands.

(C) Bart H. Husken, 2009.

This thesis can be downloaded from www.photonicbandgaps.com and www.amolf.nl.

Cover: Microscope image that shows optical Bragg diffraction from a titania inverse opal photonic crystal.

Printed: Gildeprint drukkerijen B.V.,

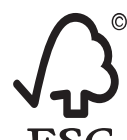
Enschede, The Netherlands.

ISBN: $\quad$ 978-90-365-2826-9

DOI: $\quad 10.3990 / 1 / 9789036528269$ 


\section{SPONTANEOUS EMISSION \\ OF NEAR-INFRARED QUANTUM DOTS \\ CONTROLLED WITH PHOTONIC CRYSTALS}

PROEFSCHRIFT

ter verkrijging van

de graad van doctor aan de Universiteit Twente,

op gezag van de rector magnificus,

prof. dr. H. Brinksma,

volgens besluit van het College voor Promoties

in het openbaar te verdedigen

op vrijdag 8 mei 2009 om 16.45 uur

door

Bart Hennie Husken

geboren op 13 oktober 1977

te Enschede 
Dit proefschrift is goedgekeurd door:

prof. dr. W. L. Vos 
To all my teachers 



\section{Contents}

1 Introduction 1

1.1 Control of spontaneous emission of light $\ldots \ldots \ldots \ldots \ldots$

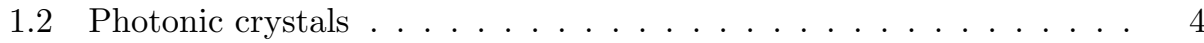

1.3 Light sources . . . . . . . . . . . . . . . . . 6

1.4 Overview of this thesis $\ldots \ldots \ldots \ldots \ldots$

2 Setup for experiments at near-infrared and visible wavelengths 11

2.1 Introduction . . . . . . . . . . . . . . . . 11

2.2 Requirements and specifications . . . . . . . . . . . . . 12

2.2 .1 Summary of requirements . . . . . . . . . . . . . 12

2.2.2 Experiments on structures with external light sources $\ldots . .12$

2.2.3 Experiments on structures with embedded light sources . . . 14

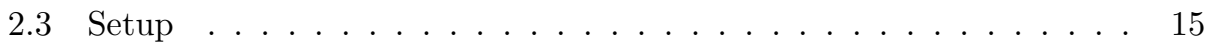

2.3.1 Implementation of experiments . . . . . . . . . . . 15

2.3 .2 Hardware configuration . . . . . . . . . . . . . . 17

2.3.3 Software configuration . . . . . . . . . . . . . . . . 21

2.4 Performance and discussion . . . . . . . . . . . . . . . . 23

2.5 Conclusions . . . . . . . . . . . . . . . . . . . 29

3 Properties of PbSe quantum dots 31

3.1 Introduction . . . . . . . . . . . . . . . . 31

3.2 Fabrication and efficiency of quantum dots . . . . . . . . 32

3.3 Excitation of single excitons in quantum dots . . . . . . . . . . 34

3.3 .1 Overview .................... 34

3.3.2 Determination experimental excitation conditions . . . . . . 35

3.4 Spectral properties . . . . . . . . . . . . . . . 37

3.4.1 Absorption measurements . . . . . . . . . . . . . . . 37

3.4.2 Emission spectra and Stokes shift . . . . . . . . . . . . 40

3.4.3 Homogeneous vs inhomogeneous linewidth . . . . . . . . . . 41 
3.5 Time-resolved emission and oscillator strength . . . . . . . . . 45

3.6 Conclusions and remarks . . . . . . . . . . . . . . . . . . 47

4 Angular redistribution of near-infrared emission from quantum $\begin{array}{ll}\text { dots in } 3 \mathrm{D} \text { photonic crystals } & 49\end{array}$

4.1 Introduction . . . . . . . . . . . . . . . . . . . . . . . 49

4.2 Experimental details . . . . . . . . . . . . . . . . 50

4.2.1 Fabrication and characterization of the photonic crystals . . . 50

4.2.2 Quantum dot infiltration and preparation for measurements . 55

4.2.3 Emission setup and measurement procedure . . . . . . . . 55

4.3 Results and discussion . . . . . . . . . . . . . . . . . . . 58

4.3.1 Emission spectra and reproducibility . . . . . . . . . . 58

4.3.2 Angle-resolved emission measurements . . . . . . . . . . . . . 61

4.3.3 Expanded escape-function model . . . . . . . . . . . . 65

4.3.4 Comparison experiment with model . . . . . . . . . . 66

4.3.5 Discussion: comparison between results and literature . . . . 71

4.4 Conclusions . . . . . . . . . . . . . . . . . . 71

5 Time-resolved emission experiments at near-infrared wavelengths 73

5.1 Introduction . . . . . . . . . . . . . . . . 73

5.2 Time-correlated single-photon counting in the near infrared . . . . . 74

5.2 .1 Experimental setup . . . . . . . . . . . . . . . 74

5.2.2 Single-photon counting statistics . . . . . . . . . . . 75

5.3 Data analysis ......................... 78

5.3.1 Raw decay-curve measurement . . . . . . . . . . . . 78

5.3.2 Evaluation of goodness of fit: use $\chi_{\text {red }}^{2} \ldots . . . . . .880$

5.3.3 Time range and bin width used for modeling . . . . . . . . . 83

5.3.4 Selection of decay-curve model . . . . . . . . . . . . . . 83

5.3.5 Outcome decay curve model: a parameter study . . . . . . 87

5.3.6 Average and variance of decay-rate distribution . . . . . . . . 88

5.4 Conclusions . . . . . . . . . . . . . . . . . . . . . . 90

6 Spontaneous emission rates of near-infrared quantum dots controlled with photonic crystals $\quad 93$

6.1 Introduction . . . . . . . . . . . . . . . . . . . 93

6.2 Experimental details . . . . . . . . . . . . . . . . . . . . . . . . . . . . . . . . . 94

6.3 Results and discussion . . . . . . . . . . . . . . . . . . . . . . 96

6.3.1 Decay curves measurements . . . . . . . . . . . . 96

6.3.2 Temporal change of measured decay curves . . . . . . . . . 97

6.3.3 Inhibition and enhancement of spontaneous emission rate versus lattice parameter and wavelength . . . . . . . . . . . . 99

6.3.4 Comparison with experimental results from the literature . . 102

6.3.5 Comparison with theory . . . . . . . . . . . . 106

6.4 Conclusions . . . . . . . . . . . . . . . . . . . 108 
7 Realignment to nanostructures in different experimental setups 111

7.1 Introduction . . . . . . . . . . . . . . . . . . . 111

7.2 Realignment method . . . . . . . . . . . . . . . . . 112

7.3 Results and discussion . . . . . . . . . . . . . . . 115

7.4 Application of realignment procedure to study opal layer growth . . 119

7.5 Conclusions . . . . . . . . . . . . . . . . . 123

A List of hardware used in the near-infrared microscope 125

B Derivation of exciton creation probability 129

C Fast infrared diode 133

D Supplementary information $\mathbf{1 3 5}$

D.1 Titania inverse opal infiltration with PbSe quantum dots . . . . . . . 135

D.2 Correction for angle dependent emission collection efficiency . . . . . 137

D.3 Parameters and results for first and second moment calculation . . . 138

$\begin{array}{ll}\text { Samenvatting } & 139\end{array}$

$\begin{array}{ll}\text { Dankwoord } & 145\end{array}$

$\begin{array}{ll}\text { Bibliography } & 148\end{array}$ 



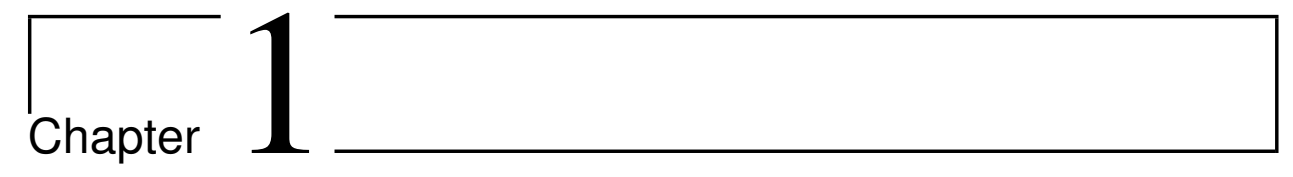

\section{Introduction}

\subsection{Control of spontaneous emission of light}

Light sources are everywhere around us and are essential to life on earth. The control of light sources, e.g., using dielectric nanostructures is very important for practical applications. Emission control can improve the efficiency of light sources, which is of general interest for lighting LEDs and miniature lasers $[1,2]$. In solar cell applications, photonic crystals are already used to increase the energy collection efficiency $[3,4]$. Furthermore, threshold-less lasers are envisioned and light sources may form the bits of the future in quantum computing applications [5].

It is known that a light sources' emission rate and wavelength are not solely determined by intrinsic properties of the light source, but also by the environment that surrounds the source [6]. Consequently it is possible to change the properties of an emitter via its environment. Control of spontaneous emission in this thesis means the control over the emission properties of a light source by changing the environment of this source. To understand what causes the emitter to change its properties requires fundamental knowledge about the interaction between matter and light. Therefore, light sources are intensively studied in different environments.

A light-emission event consists of a transition of an exited light source to a lower energy state under the simultaneous emission of a photon into an electromagnetic field mode. To describe the emission dynamics of the light source in detail requires the distinction between two regimes: the weak coupling and the strong coupling regime. The weak coupling regime is the most common situation where the electromagnetic field experienced by the emitter is not affected by the emission or absorption of its photon. Therefore, the emitter and the field with their corresponding eigenmodes can be treated separately to describe the system. It appears that these systems have no memory of their past and are known as of the Markov type [7]. In the very interesting strong coupling regime the emitter and field cannot 
be treated separately and form eigenmodes that are atomic and field-like at the same time [8-11]. Such a system does have a memory of its past and is known to be non-Markovian.

The weak coupling regime describes the transition rate between different emitterfield states $[10,12,13]$. A perturbation is required in order for a system to change from an initial state $|i\rangle$ with energy $E_{\mathrm{i}}$ to a final state $|f\rangle$ with energy $E_{\mathrm{f}}$. This perturbation originates from the interaction between an emitter and an electromagnetic field mode, which is given by the inner product of the electronic transition dipole moment $\boldsymbol{d}$ of the emitter and the electric field $\boldsymbol{E}(\boldsymbol{r})$, at the position $\boldsymbol{r}$ of the emitter. Assuming this interaction to be weak allows for the use of first order perturbation theory to derive the radiative decay rate $\gamma_{\mathrm{fi}}$ of an emitter. The result is known as Fermi's Golden Rule:

$$
\gamma_{\mathrm{fi}}(\boldsymbol{r})=\frac{2 \pi}{\hbar^{2}} \sum_{|f\rangle}|\langle f|\hat{\mu}(\boldsymbol{r}) \cdot \hat{\boldsymbol{E}}(\boldsymbol{r})| i\rangle|^{2} \delta\left(E_{\mathrm{f}}-E_{\mathrm{i}}\right),
$$

where $\hbar$ is the reduced Planck constant, $\hat{\mu}(\mathbf{r})$ is the electric transition dipole operator, and $\hat{\boldsymbol{E}}(\boldsymbol{r})$ is electric field operator. Written in this form the operator $\hat{\mu}(\boldsymbol{r}) \cdot \hat{\boldsymbol{E}}(\boldsymbol{r})$ is clearly recognized, which maps the initial state onto the final state. Furthermore, the emission decay rate is a sum of the coupling from one initial state to all final states under the condition of energy conservation. More conveniently for nanophotonics, the Golden Rule of Fermi can be rewritten in the form ${ }^{\mathrm{i}}$ [15].

$$
\gamma\left(\boldsymbol{r}, \boldsymbol{e}_{\mathbf{d}}, \omega_{g e}\right)=\frac{\pi \omega_{g e}}{3 \hbar \epsilon_{0}}|\langle g|\hat{\mu}| e\rangle|^{2} N_{\mathrm{rad}}\left(\boldsymbol{r}, \boldsymbol{e}_{\mathrm{d}}, \omega_{g e}\right),
$$

where $|e\rangle$ and $|g\rangle$ are the exited state and the ground state of the emitter, which have a frequency difference of $\omega_{g e}, \epsilon_{0}$ is the dielectric permittivity of the vacuum, $\langle g|\hat{\mu}| e\rangle=d$ is the transition dipole moment, and $\boldsymbol{e}_{\mathrm{d}}$ is the orientation vector of the transition dipole. Equation 1.2 shows that the decay rate of the emitter is given by the product of the transition dipole moment squared, which is purely a property of the emitter, and the local density of optical states (LDOS) $N_{\text {rad }}$ which involves the electromagnetic fields determined by the surroundings of the emitter [16]. Hence, it is possible to change the emission rate of a light source by controlling its LDOS, and this is what we are interested in in this thesis.

Figure 1.1 shows three examples of nanostructures that can be used to change the LDOS with respect to the vacuum density of states. Figure 1.1.(a) shows a micropillar cavity structure consisting of a cavity layer sandwiched between two Bragg reflectors [17]. Structures that exhibit optical resonances, like cavities and antennas, give rise to peaks in the LDOS which are localized in space. Such narrowband resonance peaks in the LDOS can be applied to enhance spontaneous emission

\footnotetext{
${ }^{\text {iT }}$ To derive Equation 1.1 the dipole approximation was used and first order perturbation theory was assumed to be valid. To derive Equation 1.2 additionally requires the rotating-wave approximation, the dielectric function to be real, and the interaction between the emitter and field to be of the Markov type, see Reference [14].
} 

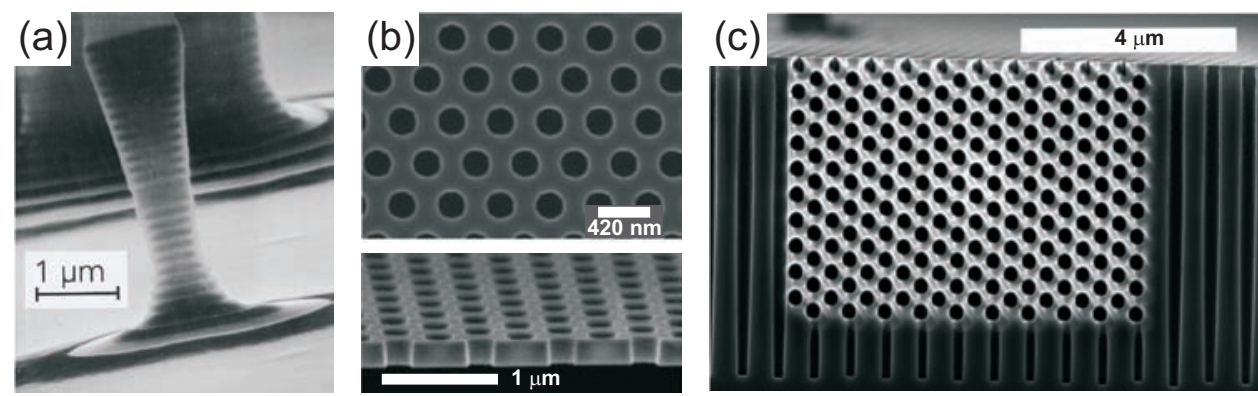

Figure 1.1: Scanning electron micrographs of three structures that strongly interact with light. (a) a micropillar resonator [17], (b) a two dimensional photonic crystal slab [18] where the top part of this figure shows a top view of this structure and the bottom part is a side view, and (c) a three dimensional photonic crystal [19]. All graphs have their own scale bar and are reproduced with permission from the authors.

of light sources inside these systems [20-27]. Figure 1.1.(b) shows a two dimensional photonic crystal that is composed of a periodic array of holes that perforate a thin layer [18]. Figure 1.1.(c) shows a three dimensional photonic crystal made of holes which were etched and focused ion beam milled into a wafer [19]. In contrast to the localized, narrow-band resonators, an emitter inside a photonic crystal couples to an infinite number of spatially extended Bloch modes. The LDOS of these crystals exhibit broad crests and troughs as a function of frequency [28, 29]. In three dimensional photonic crystals it is even possible to create a photonic band gap. Inside the band gap the LDOS is zero. This is a very special situation which allows for the complete inhibition of spontaneous emission of light sources [1]. Hence, an emitter with its transition frequency in such a band gap remains trapped in the excited state. Furthermore, the translational symmetry of photonic crystals allows for the positioning of the emitter in any unit cell. Therefore, photonic crystals enable broadband control of spontaneous emission rates over large volumes of emitters [30$36]$.

The above experiments with photonic crystals were all in the weak coupling regime, described by Fermi's Golden Rule. Recently, there have been many efforts to break the weak coupling limit, motivated by the new physics that may be studied [37-50]. As mentioned, the weak coupling regime is described by first order perturbation theory. Hence, to break the weak coupling limit one should increase the perturbation term $d N_{\text {rad }}$ such that higher order terms can no longer be disregarded in an emitters' transition rate. There are several ways to increase this emitter-field perturbation term: (1) by increasing the field at the position of the emitter, e.g., using cavity structures, (2) by use of Van Hove singularities, which are revealed as sharp cusps in the LDOS and that may appear at the edge of a band gap [14, 51], 
(3) by careful selection of an emitter with a large transition dipole (or oscillator strength), and (4) by the careful positioning and orientation of the emitter and field such that the inner product in the perturbation term becomes maximum [42]. (5) An alternative to the first two items is to rapidly change the LDOS in time [52]. Photonic crystals with a band gap are very interesting for these studies as the band gap reduces the LDOS to zero over a frequency range. As a consequence the LDOS at the edge of this band gap will exhibit a very steep increase with frequency. The same holds for resonance peaks that arise from fabricated defect modes inside the structure.

Therefore, in the pursuit of spontaneous emission control, to the point of strong coupling, photonic crystals with a band gap are very interesting nanostructures for several reasons. In bulk crystals, sharp cusps are expected at the edges of the gap. Point defects embedded in band gap crystals act as resonators with a strongly enhanced LDOS; the vanishing background LDOS is favorable as it minimizes vacuum noise; in particular in three dimensional crystals the quality factor of an embedded resonator is expected to increase exponentially with crystal size [53, 54].

It is for these reasons that we have embarked on a study of light sources in strongly interacting photonic crystals. Since we ultimately aimed at photonic crystals with embedded point defects, we also had to develop methods to realign to deterministically fabricated defects, as well as develop a dedicated microscope setup.

\subsection{Photonic crystals}

Photonic crystals are composite materials with a periodic change in the dielectric function on length scales that are comparable to the wavelength of light. Light inside these structures propagates in Bloch modes which have the form of a plane wave times a function with the periodicity of the crystal [55]. Bragg diffraction of the light occurs if the wavelength of the light is approximately equal to the periodicity of the lattice planes inside the structure, which occurs at the Bragg condition [56-58]:

$$
m \lambda=2 d \cos (\theta)
$$

with $\lambda$ the Bragg diffracted wavelength, $m$ an integer that corresponds to the order of the diffraction ( 1 in most studies), $d$ the distance between the lattice planes and $\theta$ the angle at which the light propagates with respect to the surface normal of the lattice planes, see Figure 1.2.(a). Instead of considering the wavelength of the light it is more convenient to use the dispersion relations of the photonic crystal, that describe the relation between the frequency of the light $\omega$ and the wave vectors $\boldsymbol{k}$. For low frequencies, the dispersion relation is $\omega=c|\boldsymbol{k}|$, where $c$ is the velocity of light. At wavelengths equal to the Bragg condition $(|\boldsymbol{k}|=\pi / d)$ the dispersion relation opens up and reveals a stop gap over a frequency range $\Delta \omega$, which is centered around the frequency $\omega_{0}$ that fulfills the Bragg condition, as illustrated in Figure 1.2.(b). In the frequency range of the stop gap the photonic crystal does not 
(a)

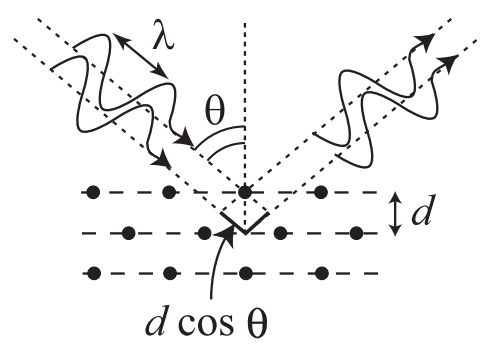

(b)

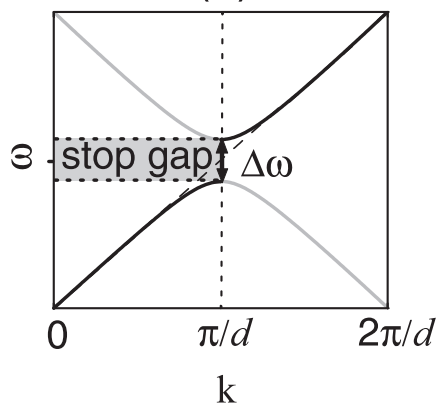

Figure 1.2: (a) Illustration of Bragg's law, Equation 1.3. The light reflected from lattice planes that are spaced by a distance $d$ constructively interferes when, for an incident angle $\theta$, the optical path difference $2 d \cos (\theta)$ equals an integer number of incident wavelengths $\lambda$. (b) Dispersion relation between the frequency $\omega$ and the wave vector $\boldsymbol{k}$ inside a photonic crystal. At low frequencies $\omega$ is linearly dependent on $|\boldsymbol{k}|$, whereas at the Bragg condition $(|\boldsymbol{k}|=\pi / d)$ a stop gap opens for which the structure does not support any modes.

support propagating modes.

The relative width of the stop gap can be written as $[15,56,59]$

$$
S=\frac{\Delta \omega}{\omega_{0}}=4 \pi \frac{\alpha}{v} \approx \frac{|\Delta \epsilon|}{\bar{\epsilon}}\left|f_{\mathrm{G}_{\mathrm{hk} 1}}\right| .
$$

Here $S$ is the photonic strength which is defined as $4 \pi$ times the polarizability $\alpha$ per unit volume $v$ of each scattering building block [56]. This parameter is a general gauge of the interaction between light and any nanophotonic structure. $\Delta \epsilon$ is the dielectric contrast between the materials in the photonic crystal, $\bar{\epsilon}$ is the volume averaged dielectric constant, and $f_{\mathrm{G}_{\mathrm{hk}}}$ the Fourier component associated with the reciprocal lattice vector $\mathrm{G}_{\mathrm{hkl}}$ (in FCC structures like opals we are typically interested in $\left.\mathrm{G}_{111}\right)$. To obtain a crystal with a photonic band gap requires the simultaneous coupling of Bragg diffraction conditions [60]. Therefore, a large photonic strength is needed in combination with a suitable crystal geometry. Clearly this is what we need to achieve for the experiments discussed in the previous section.

From Equation 1.4 it is clear that there are in essence three ways to increase the photonic strength of a photonic crystal [15]: (1) by increasing the dielectric contrast of the constituents $\Delta \epsilon,(2)$ by reducing the average dielectric constant of the structure $\bar{\epsilon}$, and (3) by optimization of the unit cell geometry of the crystal to enhance $f_{\mathrm{G}_{\mathrm{hkl}}}$. The most promising structures with the largest band gap are expected to be inverse woodpile structures that have a diamond like structure ${ }^{\mathrm{ii}}[61]$.

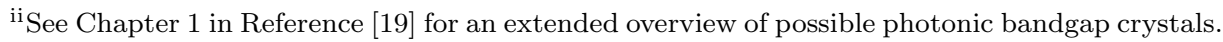


These structures, when made of silicon and air have broad predicted bandgaps of $\Delta \omega / \omega \approx 25 \%$ [61-63]. In addition, silicon is a very popular material used in many applications and in CMOS industry. These silicon-air photonic crystals are currently being developed in our group, and Figure 1.1.(c) shows a recent result [19].

The use of silicon for photonic crystals implies a major constraint on the emitters that can be used in our studies. Silicon has a relatively small electronic band gap of $E_{\text {gap }}=1.12 \mathrm{eV}$. Consequently, silicon is only transparent at wavelengths longer than $\lambda=1100 \mathrm{~nm}$. Therefore, our aim is to control emission rates at wavelengths $\lambda>1100 \mathrm{~nm}$. Since this range happens to include the telecom-range, our results might also have relevance for this field.

As mentioned, the photonic crystal influences the emission rate of a light source via the LDOS (Equation 1.2). For strongly photonic crystals $(S \geq 0.10$ [15]), the LDOS changes dramatically with frequency, for frequency-ranges that correspond with the band gap or with broad stop gaps. At low frequencies the LDOS follows the smooth $\omega^{2}$ dependence that is known for the density of states in homogeneous systems $[14,35]$. At these frequencies optical Bragg diffraction does not occur and therefore the crystal is referred to as non-photonic. Such non-photonic structures form a good reference system for studies on the emission dynamics of light sources in the photonic regime [15]. Light sources embedded in photonic and non-photonic structures experience the same chemical environment and average refractive index of their surrounding material. Consequently, changes in the emission dynamics can be attributed to LDOS effects. In our studies this approach results in the comparison between the emission decay of light sources that emit a fixed frequency and are placed inside photonic crystals, with the decay of the same light sources placed in a crystal with a much smaller lattice parameter, which is thus non-photonic.

As a first step towards the intended experiments at the near-infrared frequencies we decided to use strongly photonic titania inverse opal photonic crystals, as shown in Figure 1.3, since the silicon photonic crystals were still under development at the start of this project. Inverse opals were successfully applied in the first demonstration of spontaneous emission control of light sources with photonic crystals [30]. For our study crystals were fabricated for light sources that emit at near-infrared wavelengths $\lambda>1100 \mathrm{~nm}$.

\subsection{Light sources}

The properties of the light source that we aimed for are as follows:

- The light source must emit at wavelengths $\lambda>1100 \mathrm{~nm}$ to be applicable in photonic crystals made of silicon.

- The quantum efficiency of the light source must be high to observe changes in the time-resolved decay rate due to changes in the LDOS ${ }^{\text {iii }}$.

\footnotetext{
${ }^{\text {iii }}$ Remember that the measured emission decay stems from a combination of radiative and non-radiative decay channels. The LDOS only influences the radiative part. For low quantum
} 


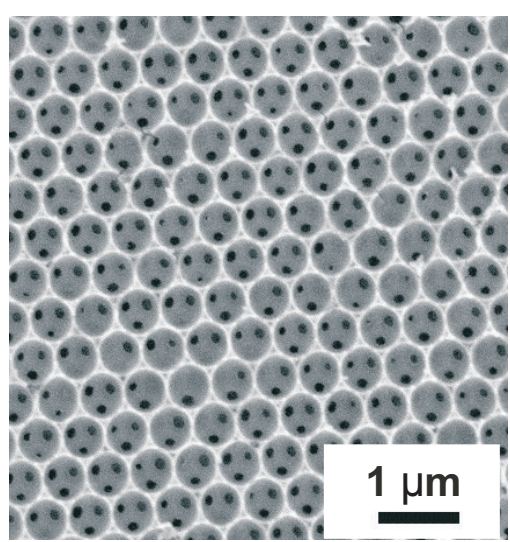

Figure 1.3: Scanning electron micrograph of titania inverse opal photonic crystal, which consists of air spheres in a titania matrix. The white structures are the titania. The dark spots are holes that connect the neighboring air spheres. These holes arise during the infiltration process that is part of the fabrication. The spheres in the opal that is infiltrated with the titania precursor touch and therefore locally prevent the development of titania. Consequently the air holes are connected by small openings.

- The radiative transition of the light source should preferably behave like a two level system to facilitate the interpretation of the experimental results.

- The emitters' homogeneous linewidth should be narrow to prevent coupling of light emitters to modes outside the band gap.

- The transition dipole moment (or similarly oscillator strength) of the emitter must be large to increase the interaction between the emitter and the electromagnetic field. In practice, the radiative decay rate of the emitter should also be large to facilitate time-resolved emission experiments. When the system under study decays fast, the time per measurement can be reduced and the experiments become considerably faster.

Possible light sources in the near-infrared range are infrared dyes, rare earth ions, and quantum dots. The dyes are know for their low luminescence quantum efficiency, broad emission spectrum, and at these wavelengths they are relatively unstable. Ions, like the widely used erbium, may exhibit high quantum efficiencies but have a low oscillator strength and the emission wavelength is fixed by the choice of ion [64]. The emission spectrum of semiconductor quantum dots is determined by quantum size confinement effects [65-68]. Therefore it is possible to fabricate a light source with a desirable emission wavelength by control of the quantum dots' size. Due to confinement effects quantum dots behave effectively as two level systems

efficiencies the emission decay is determined mostly by the non-radiative decay. Hence, at low quantum efficiencies, changes in the LDOS hardly change the total, measured emission rate. 
with a narrow emission spectrum. This makes them ideal candidates for quantum electrodynamics experiments $[37,38]$, especially since mono-disperse colloidal quantum dot suspensions are commercially available. In this thesis PbSe quantum dots will be applied.

\subsection{Overview of this thesis}

- Chapter 2 describes the experimental setup that was built for quantum dot emission experiments at near-infrared wavelengths. The specifications for the experiments are defined for the experimental approach where a sample is translated together with a cryostat. Besides the hardware we discuss software developed to automate the experiments. The setup design allows for flexible addition and removal of hardware components. In the last part of the chapter it is experimentally verified that the setup meets most intended specifications.

- Chapter 3 contains the experimental characterization of the PbSe quantum dot light sources used throughout this thesis. The absorption cross-section of the dots is determined, and both absorption and emission oscillator strengths were derived from experiment. Absorption oscillator strengths between 6.9 to 10 were found, which are approximately 7.5 times larger than the measured emission oscillator strength. We found the oscillator strength to increase with quantum dot size, giving rise to an increase of the radiative decay rate with size, also observed in Chapter 6 . The relative width of the quantum dot ensemble emission spectrum is relatively large compared to that of, e.g., CdSe quantum dots. The PbSe dot ensemble suspended in hexane is estimated to be inhomogeneously broadened by at most a factor of two, in agreement with literature. We found that the dots are suitable for use in the silicon air structures we aim for, as these structures provide a band gap that covers the emission spectrum of the dots.

- Chapter 4 discusses the angle-resolved emission of PbSe quantum dots inside inverse opal photonic crystals. Strong deviations from the Lambertian emission profile are observed. An attenuation of $60 \%$ is observed in the angle dependent power emitted from the samples, due to photonic stop bands. At angles that correspond to the edges of the stop band the emitted power is increased by up to $34 \%$. This increase is explained by the redistribution of Bragg-diffracted light over the available escape angles. The results are quantitatively explained by an expanded escape-function model that is based on diffusion theory and extended to photonic crystals.

- Chapter 5 addresses the experimental methods of time-resolved emission experiments in the near-infrared. Thereby it serves as a technical introduction to Chapter 6. Compared to the visible regime, the signal to noise ratio turns out to be more than $10^{3}$ times smaller in the near-infrared, due to the large dark signal of near-infrared detectors. Furthermore, the decay curve data analysis is discussed in detail. The large background signal plays an important role, 
especially in the calculation of the goodness of fit $\chi_{\text {red }}^{2}$. We analyze decay curves of PbSe quantum dots in inverse opals. The data are strongly nonexponential. Log-normal distributions of decay rates are shown to describe the measured decay curves accurately. We found the parameters in this model to be strongly correlated for our data. As a result different parameter sets can model the same decay curve accurately. We therefore derived the average decay rate and variance of the log-normal distribution and demonstrated that these are quantitative measures to compare different decay curves.

- Chapter 6 contains the first demonstration of control over spontaneous emission decay of quantum emitters at near-infrared wavelengths, using photonic crystals. PbSe quantum dots were placed inside 3D titania inverse opal photonic crystals. Using the methods developed in the previous Chapter 5, we found an inhibition of the emission rate up to $51 \%$ and an enhancement up to $29 \%$, as compared to the decay rates measured from reference samples. PbSe quantum dots are therefore a suitable light source for experiments in photonic band gap structures.

- Chapter 7 discusses the experimental method that was developed to recover a single, deterministically fabricated nanostructure in various experimental instruments without the use of artificially fabricated markers, with the aim to study photonic band gap structures with cavities. Therefore, a detailed map of the spatial surroundings of the nanostructure is made during the fabrication of the structure. We demonstrate how intrinsic and characteristic geometric features on a sample can be used in different setups to act as markers. Since this approach does not use artificial grids or markers, it is of particular interest for samples whose structure is not known a-priori, like samples created solely by self-assembly. In addition the method is not restricted to conducting samples. 

${ }_{\text {Chapter }} 2$

\section{Setup for experiments at near-infrared and visible wavelengths}

\subsection{Introduction}

The experiments introduced in Chapter 1 require a dedicated experimental setup that exceeded the specifications of our previous optical setups [14, 15, 69]. There is no setup available commercially that combines the required functionality and specifications, discussed below. Therefore, a new setup was designed, built and tested, which is described in this chapter.

One of the goals is to fully control the emission decay of light sources, using dielectric structures like photonic crystals and microcavities. At first, these experiments are done with ensembles of emitters. However, the aim is to reduce this ensemble to the single emitter regime to study the interaction of the light source beyond the weak coupling regime. Typical experiments include the case of an emitter strongly coupled to a resonating structure, like a cavity $[20,37,38]$, and light transport through coupled resonances [70]. These experiments are intended for wavelengths from the visible to near infrared range, i.e., $\lambda=400 \mathrm{~nm}$ to $2 \mu \mathrm{m}$.

There are basically two ways to distinguish the experiments that we have in mind: 1) experiments on structures with external light sources versus structures with embedded light sources, and 2) time-resolved measurements versus continuous-wave measurements. Hence, there are four different types of experiments that all require different specifications. The specifications for these experiments were combined in a list, which was used to select the hardware components. Furthermore, software was developed to allow for the use of a sub-selection of the available 
hardware components and to automate the data collection in the experiments.

In addition to the hardware and software requirements, one additional requirement is of general importance for all aspects considered during the development of the setup: make the setup suitable for changes. In practice this means that it must be possible to add and remove hardware components, change beam paths, and keep the setup operational under the unforeseen circumstance of component failure. Furthermore, this approach allows for the start of new experiments before the intended setup is completely finished.

In this chapter the technical aspects of the setup are discussed and a reflection on the design is given. This setup will subsequently be used in Chapters 3 to 6 . First, a list of experimental requirements is derived in Section 2.2. Before this list can be used to select adequate hardware, a decision is needed upon the practical implementation of the experiment, discussed in Section 2.3.1. Subsequently, the hardware layout of the setup is given in Section 2.3.2, and Section 2.3.3 contains the basic functionality of the software that was developed to automate the measurements. The system performance was experimentally determined as discussed in Section 2.4. Conclusions form the final part of this chapter, Section 2.5.

\subsection{Requirements and specifications}

\subsubsection{Summary of requirements}

The experimental setup needed to combine the specifications is basically a (confocal) microscope with dedicated positioning stages for sample and objective. In addition various entrance and exit ports need to be realized to probe the sample. Table 2.1 summarizes the requirements from the discussion in the following sections.

\subsubsection{Experiments on structures with external light sources}

Before the interaction between emitters and dielectric nanostructures is studied, these structures must be characterized. Therefore, reflectivity and transmission measurements are needed. As the temperature can be used as a parameter to change the samples' properties, this temperature needs to be controlled, see for example $[37,38,71,72]$. An additional survey of the sample is required to realign the sample such that the setup probes the same location in different experiments, as discussed in Chapter 7.

Reflectivity and transmission experiments require basically three things: 1) a light source, 2) a dispersive element, and 3) a detector, where the dispersive element and detector together form a spectrometer. To determine the position and width of a photonic crystals' stop band or band gap the measured spectral range must be larger than this gap. In strongly photonic silicon-air structures these gaps may have relative widths $\frac{\Delta \omega}{\omega}$ up to $25 \%[15,62,73]$, where $\Delta \omega$ is the width and $\omega$ the central frequency of the gap. Hence, to resolve the gaps in reflectivity and 


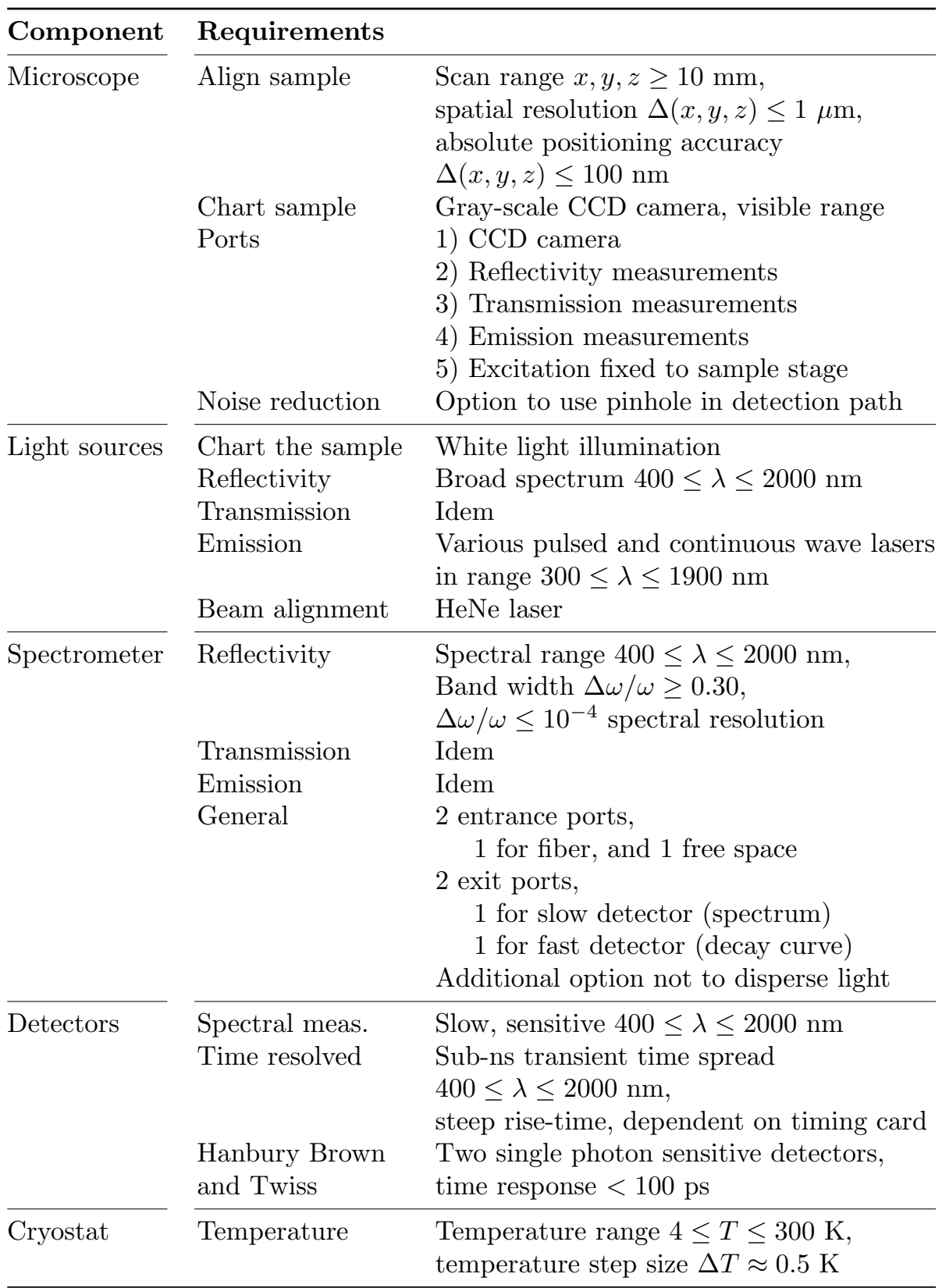

Additional requirement: make the setup suitable for changes

Table 2.1: Summary of experimental requirements for the setup. Left column describes the system component, the middle column contains a keyword to what the requirement in the third column refers to. 
transmission measurements, a broadband light source is needed in combination with a spectrometer that can measure over a band width of at least $\frac{\Delta \omega}{\omega}>0.25$.

On the other hand a high spectral resolution is required in case of experiments on single emitters and a high quality cavity mode (inside strongly photonic systems). As a measure for the quality factor $\mathrm{Q}$ of these cavities the relative width in frequency is often used ${ }^{\mathrm{i}}$. Q factors of about $10^{4}$ are typical for these experiments together with relative mode separations of $\frac{\Delta \omega}{\omega}=1.7 \cdot 10^{-4}[38]$ or $\frac{\Delta \omega}{\omega}=1.1 \cdot 10^{-4}$ [37]. Hence, spectral resolutions of at least $\frac{\Delta \omega}{\omega}<10^{-4}$ are needed. Recently our group has started to work on cavities with quality factors in excess of 1000 [76].

To probe small structures, and later single emitters, requires precise alignment. Microscope objectives are needed to focus light on the structure and collect reflected or transmitted light ${ }^{\mathrm{ii}}$. A diffraction limited spot has a diameter $D$ approximately given by the ratio between the wavelength of the light and the numerical aperture of the objective, i.e., $D \approx \lambda / \mathrm{NA}$ (see Page 130 ). In the visible range this leads to typical diameters of about $1 \mu \mathrm{m}$, which can be used as a measure for the spatial resolution of the setup. To position the sample such that the beam focus is centered on top of a cavity or emitter therefore requires sub-micron positioning control; $0.1 \mu \mathrm{m}$ is used as a practical guide for the alignment. Samples have typical lateral extents of $1 \mathrm{x} 1 \mathrm{~cm}$ and a few millimeters thick. Therefore a scan-range larger than $10 \mathrm{~mm}$ is needed to align to the region of interest, using the samples' boundaries for reference.

\subsubsection{Experiments on structures with embedded light sources}

In spectroscopic emission measurements, the aim is to measure a complete emission spectrum at once, using an array or a ccd detector. Large relative spectral linewidths of emitters are expected due to inhomogeneous broadening processes. The quantum dot ensembles used in this thesis reveal a relative emission linewidth of $15 \%$. A spectral range of at least two times this width is therefore useful for experiments, hence, $\frac{\Delta \omega}{\omega}>0.30$. Similar to the previous section relative widths for single quantum dot emission are expected to be on the order of $10^{-4}$ or larger [7880], which corresponds to the spectral resolution required in the previous section.

Time-resolved experiments are needed to demonstrate the control of emission decay of light sources. The time-correlated single photon counting technique is used for these measurements, see Chapters 5 and 6 for details ${ }^{\mathrm{iii}}$. Basically these experiments require a detector with single-photon sensitivity, and a timing card. Furthermore, the combination of detector and timing card needs to be fast with

\footnotetext{
${ }^{\mathrm{i} N o t e}$ that in principle the $\mathrm{Q}$ factor is a property not only of the cavity, but also of the incident field by which the cavity is probed [74]. In contrast to relative linewidth measurements the cavity Q can be determined from ring-down experiments [75], which are outside the scope of this text.

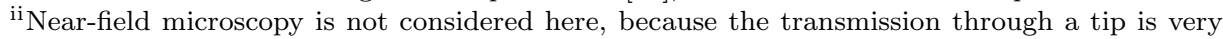
low [77].

iii To apply pump-probe experiments to measure the lifetime is practically impossible for large lifetime ranges. Path length differences of hundreds of kilometers would be needed to probe millisecond lifetimes.
} 
respect to the lifetime of the emitter. In the visible and near-infrared range measured lifetimes typically span a range from about a nanosecond up to a millisecond $[14,20,21,24,25,27,30,31,81-84]$. For the visible range we already posses a system to measure lifetimes with 55 ps time resolution, which was successfully coupled to the setup using an optical fiber (for the emission signal) and a coaxial cable (to get the detectors output signal). Therefore, mainly a detector suitable for time-resolved measurements in the near-infrared region is required.

In addition to these time-resolved measurements, in future we wish to verify that the observed emission results from single emitters. Therefore, a Hanbury Brown and Twiss (HBT) interferometer can be used to measure the autocorrelation function $g^{(2)}\left(t^{\prime}\right)=\left\langle I(t) I\left(t+t^{\prime}\right)\right\rangle /\langle I(t)\rangle^{2}$, with $I(t)$ the measured intensity at time $t^{\text {iv }}$. The coincidence rate between the two detectors in this interferometer should reveal antibunching behavior at time $t^{\prime}=0$ if the emission stems from a single emitter, see for example References $[24,80,85-87]$. Single-photon sensitive detectors with response times of about an order of magnitude faster than the lifetime of the studied emitter are needed to measure antibunching. Given the minimum lifetime of one nanosecond mentioned above, the fast detector is required to respond in $100 \mathrm{ps.}$

Especially for single emitter measurements high collection efficiencies and good signal to noise ratios are required. Therefore a spatial filter can be applied in the optical path to the detector to block the light emitted outside the volume of interest. Furthermore, single emitter experiments are mostly done at low temperatures of typically 5 to $100 \mathrm{~K}$, to improve the quantum efficiency of the source and reduce the inhomogeneous broadening. A cryostat is needed to reach these low temperatures and keep the sample at a constant temperature. Typically, temperature resolutions of about $0.5 \mathrm{~K}$ are applied in low temperature experiment on single emitters [37, 38]. At present we posses a cryostat, although it is of limited use to microscopy.

\subsection{Setup}

\subsubsection{Implementation of experiments}

Before the list in Table 2.1 can be used to select adequate hardware, we need to decide upon the practical implementation of the experiment. There are various ways to accomplish the area scans needed to compare emission, transmission, or reflectivity results. Depending on the approach, hardware components that meet the requirements (Table 2.1) may be suited. The options considered for making area scans of sample surfaces can be divided into four groups, namely:

1. Sample scanning

Stages inside the cryostat are used to translate the sample through the detection/excitation focus. During the experiment only the sample moves, and the cryostat and detection path is unaltered [88].

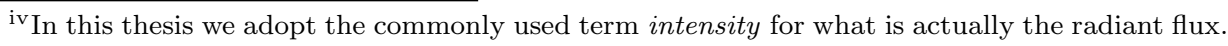




\section{Beam scanning}

Use steering mirrors to guide a beam at different angles through an objective to illuminate and probe different positions on the sample. During the experiment the sample and cryostat remain at the same position, and the detection path is unaltered. This technique results in typical scan ranges of $100 \times 100 \mu \mathrm{m}^{2}$. All major microscope companies have this option available as a modular scan head that can be mounted on a side port of a microscope. Home-built systems are also widely used, see for example Reference [89, 90]. In this experiment the sample and cryostat remain at a fixed position and the detection path is unaltered.

3. Objective scanning

Scan the objective above the sample to change the position of the detection and excitation foci. During the experiment the sample and cryostat remain at the same position, but the detection path is slightly altered.

\section{Cryostat scanning}

Move the cryostat together with the mounted sample through the detection and excitation foci of the setup. Hence, the sample and cryostat move, while the detection path stays the same.

The first option is not considered to be a suitable choice for our experiments, mainly because the measurements must be done over a large temperature range. There are stages available that work at cryogenic temperatures. However, these stages behave differently at different temperatures. Consequently, the setup needs to be calibrated at each temperature used. In addition a good and flexible thermal contact between the sample and the cold-finger of the cryostat needs to be developed. Furthermore, this option requires a bulky cryostat that may hinder different types of experiments, as it is relatively demanding to remove it from the experiment.

The second option is widely used, especially in cell-biology and single-molecule detection. Although this technique is successfully applied for single molecule experiments [91] the spectral range specified in Table 2.1 results in the necessity to change detection sensitivity from near-infrared wavelengths to the visible regime, and back. Commercially available scan-heads are specifically designed only for the visible range. The scan head contains many glass components and anti-reflection coatings that make it opaque for near-infrared frequencies. Furthermore these heads are designed for inverted microscopes where we preferred to use an upright microscope experiment, as in some cases it may not be possible to mount a sample upside down. Hence, home-built systems would be the only option. Application of this technique results in excitation and detection paths that are guided away from the central optical axis while chromatic abberations are known to play an important role in these systems. Therefore, wavelength changes are expected to become very demanding, as large parts of the experimental setup will have to be realigned. Therefore this option was excluded. 
The third option is very straightforward and also used successfully [91]. However, beforehand it is difficult to asses by how much the detection efficiency changes when the objective is moved away from the optical path. Clearly, additional stages are needed to reach the $10 \mathrm{~mm}$ translation range (Table 2.1).

Finally the fourth option is expected to be most straightforward from the optics point of view: nothing changes in the illumination or detection path. Moving around a large cryostat with its flexible transfer tubes may give rise to unforeseen vibrations in the setup. Because of the weight of the cryostat (minimally about two kilograms) and the required positioning accuracy of $100 \mathrm{~nm}$, it may not be obvious that this concept works. However, these systems are already used successfully with $1 \mu \mathrm{m}$ absolute alignment accuracy in combination with $25 \mathrm{~mm}$ translation range $\mathrm{e}^{\mathrm{v}}$. However, there is no Z-stage available that can bear the heavy cryostat and be positioned with the same accuracy. Therefore an additional Z-stage is needed for the objective.

To conclude, the first two options are considered too demanding and impractical given the requirements from Table 2.1. Both options three and four look very promising, but need additional stages. Therefore, options three and four are combined. Hence, a microscope cryostat is mounted on an automated XY translation stage with $100 \mathrm{~nm}$ absolute positioning accuracy. This combination is mounted on a rigid labjack used for coarse Z-alignment. The objective is placed in an XYZ objective stage. It is expected that the XY stage combination that moves the cryostat is sufficiently good for the area scan. In case the movement of the cryostat causes too much vibrations in the system, this XY combination is only used for coarse alignment and the XYZ objective stage will be used to make the area scan.

\subsubsection{Hardware configuration}

The experimental setup and its legend are shown in Figures 2.1 and 2.2, respectively.

The central part of the setup is the sample, depicted by the star at the bottom of the image. Black components and lines correspond to the configuration of the setup, as used in Chapters 3 to 6 . Gray components and lines were not used in the present thesis, disregarding the gray flip-mounted mirrors. Basically, the left part of the figure is used to superimpose all light sources for pulsed excitation or broadband transmission or reflectivity measurements with the same optical path. The bottom right part is the microscope with the sample, its white light illumination, stages, objective lenses and camera. The stages have sensors for absolute positioning control to reduce drift during the experiments. The objective above the sample is mounted as in a normal upright microscope. The upper right part is formed by the detection facilities. Centrally, a gray dotted square marks the part of the setup that is raised with respect to the optical table, using a breadboard on four posts. The horizontal dashed line shows part of the optical path below this breadboard. Optical paths are

\footnotetext{
${ }^{\mathrm{v}}$ We are very grateful to Takashi Kuroda Ph.D., Prof. Bennet B. Goldberg, and Richard D. Younger Ph.D. for sharing their expertise on microscope cryostat scanning experiments.
} 


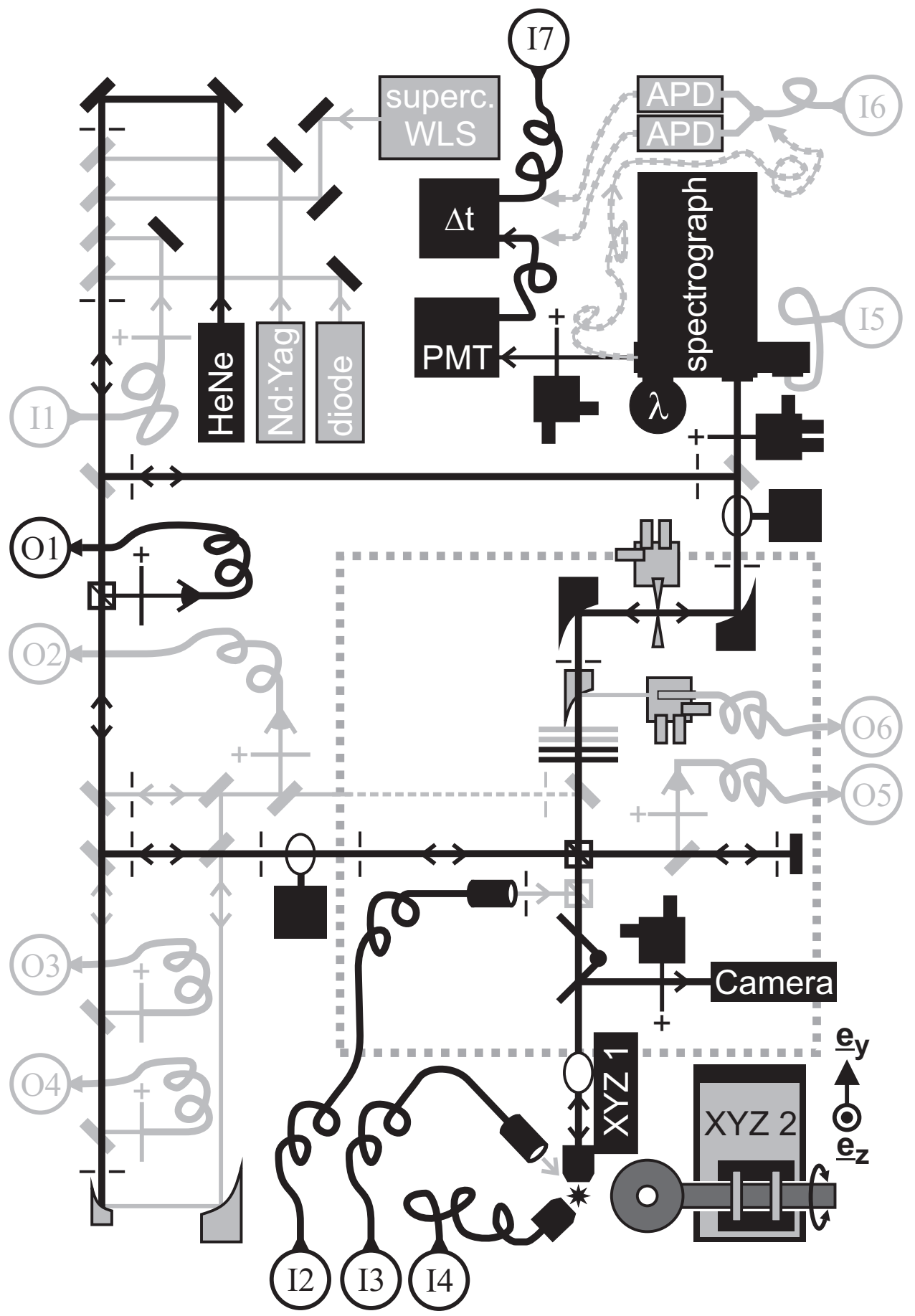

Figure 2.1: Schematic representation of the experimental setup. Legend on Page 19, clarification in Section 2.3.2, and details on components in Appendix A. 


\section{Legend:}

* sample location

microscope objective

optical beam with given propagation direction

mirror

mirror on flip mount

parabolic mirror

beamsplitter

beamsplitter with glass
correction

$+\longrightarrow$ positive lens

- - iris diaphragm
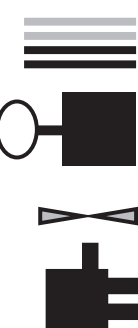

long pass filters

$850 \mathrm{~nm}$ and $1100 \mathrm{~nm}$

periscope

pinhole

(currently not used)

xyz translation stage

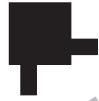

$x y / x z$ translation stage

different diode detectors

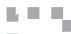

-

raised breadboard

labeled (O5) outgoing signal, fiber or coax. labeled (I6) incoming signal, fiber or coax.
$\mathrm{HeNe}$

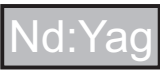

diode

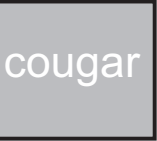

\begin{tabular}{|c|}
\hline superc. \\
WLS \\
\hline
\end{tabular}

Camera

PMT

APD

$\Delta \mathrm{t}$

XYZ 1 XYZ piezostage

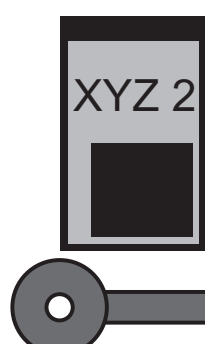

automated $X Y$ stage plus manual $Z$, optional addition of rotation stages

microscope cryostat grating spectrometer, double entrance/exit port, includes 1024 element liquid nitrogen cooled line detector for near-infrared

Figure 2.2: Legend to Figure 2.1, details on components in Appendix A. 


\begin{tabular}{|c|c|}
\hline Input port & Description \\
\hline I1 & $\begin{array}{l}\text { Lensed optical fiber to couple in laser light from different ex- } \\
\text { perimental setups }\end{array}$ \\
\hline $\mathrm{I} 2+\mathrm{I} 3$ & Fiber halogen lamp for white light illumination \\
\hline I4 & $\begin{array}{l}\text { Lensed optical fiber to couple in laser light from different ex- } \\
\text { perimental setups }\end{array}$ \\
\hline I5 & Dedicated triple core optical fiber for side entrance spectrometer \\
\hline I6 & 50-50 optical fiber multiplexer \\
\hline I7 & Electronic reference signal of pulsed excitation lasers \\
\hline Output port & Description \\
\hline $\mathrm{O} 1$ & $\begin{array}{l}\text { Electronic signal of fast near infrared diode (sma connection) } \\
\text { (see Appendix C for details) }\end{array}$ \\
\hline $\mathrm{O} 2$ & Electronic signal from switchable gain, amplified silicon detector \\
\hline $\mathrm{O} 3$ & Lensed optical fiber, single mode for $\lambda=1064 \mathrm{~nm}$ \\
\hline $\mathrm{O} 4$ & Lensed optical fiber, single mode for $\lambda=532 \mathrm{~nm}$ \\
\hline O5 & Electronic signal from switchable gain, amplified silicon detector \\
\hline O6 & Optical fiber to transmit light directly to different spectrometers \\
\hline
\end{tabular}

Table 2.2: Clarification of input and output signals as shown in the drawing of the experimental setup (Figure 2.1). The I- and O-numbers in the left column correspond to numbers in the drawing.

defined by iris diaphragms. The vertical black line which ends at the bottom left of the figure depicts the beam path used to overlay the light sources. Flip-mounted mirrors are used to guide the light to different optical paths, or couple into an optical fiber. Arrows in the beam path point along the direction of light propagation of the light. Most paths are used in two ways for alignment purposes, using the upper left iris in combination with other irises as references. At the outer rim of the setup encircled numbers label the incoming (I) and outgoing (O) signals, explained in Table 2.2. Product names and brands of the hardware are listed in Appendix A. Electrical signals from the fast detectors are transmitted through sma-type cables and connectors, while slow detectors are connected to the setup using regular coax cables and BNC connectors.

Mostly enhanced aluminum mirrors are applied, as these are highly reflecting for both the visible, and near-infrared regions. Only the spectrograph in Figure 2.1 has a gold-coating on its mirrors and one grating, as this gives better performance at near-infrared wavelengths.

Not shown in Figure 2.1 is the connection to the hardware controllers and central computer. As mentioned, the setup should be flexible to changes. Furthermore, it is clear that many components should be controlled by the same computer. There- 
fore, PCI-connection boards are used wherever possible to control the hardware components. These boards are placed in a separate 13-slot PCI to PCI Expansion System, which is connected to the computers PCI-bus using a bridge construction. This approach should make it easy to use the setup in the future, even when the main computer needs to be replaced for a new one that contains less PCI, or USB connections. Furthermore, easy access to the expansion slots simplify the addition or removal of components from the setup. The only exception to this PCI approach is the CCD-camera. This firewire camera is connected to one of the computers PCIExpress slots with a separate card. This is done to speed up the camera-readout and prevent noise on the PCI-bus (for which ccd cameras are known). As the processor capacity is divided over the PCI-bus and the PCI-express bus via a fixed ratio, the use of an express-slot should not hinder the PCI-bus.

As an illustration of the practical use of the setup from Figure 2.1, a typical lifetime measurement from Chapter 6 is explained. The sample is inside a water and oxygen free chamber and mounted on the XYZ 2 stage. A low magnification objective is used $(\mathrm{NA}=0.05)$ in the $\mathrm{XYZ} 1$ stage, which is switched off. I3 is used to illuminate the sample while aligning the sample by viewing with the camera. Subsequently, a pulsed laser is coupled into a fiber to excite the sample via I4. Emission from the sample is collected by the objective in stage XYZ 1 and sent towards the top of the figure, passing through the dielectric mirror and the glass plate that corrects the beam distortion caused by the dielectric mirror. Long pass filters are used to block the excitation laser light. Subsequently the emitted light is reflected by two parabolic mirrors and a periscope. A lens is used to focus the light on the entrance slit of the spectrograph. The front output port of the spectrograph is used to collect an emission spectrum. Subsequently, the side exit port is used to collect a decay curve. For the decay curve measurements a reference timing signal from the laser is used via I7. The additional beam splitters, pinhole, and parabolic mirror were not mentioned because these parts were translated out of the detection path during these experiments.

\subsubsection{Software configuration}

Most hardware is computer controlled and consequently comes with own drivers and user interfaces. Additional software is needed to automate the time consuming experiments where different hardware components need to be combined. A complete description of the developed software and user interfaces is outside the scope of this thesis. Only the basic functionality is explained to clarify what was done to satisfy the requirement: make the setup suitable for changes. Therefore, the following requirements were specified for the software:

1. The software must come with a clear user interface that contains the parameters needed in the experiment.

2. It must be possible to add new hardware components. 
3. It must be possible to run experiments that use only part of the available hardware components. Hence, if one component is not installed or not needed in the experiment, the rest of the setup should remain operational.

4. The experimental settings used for the hardware components need to be stored for later reference.

5. In case the experiment is interrupted for whatever reason, it should be possible to recover the data that have been measured till the interruption.

6. During a single measurement it should be possible to view the data that was measured so far.

These requirements are met by using the following approach. Each hardware component is given its own graphical user interface (GUI) to set possible parameters and control the component ${ }^{\text {vi }}$. These interfaces enable data storage, and are completely autonomous. Hence, different interfaces with the underlying software components do not interfere. Consequently, the addition of new hardware simply means the addition of a new independent software component with its own GUI.

An additional software component, known as the manager, is used to combine different hardware in an automated experiment. These experiments are turn based, which means that the manager contains a so called experimental marshal unit (EMU) that tells the software components when it is their turn to perform a measurement step. No two components are allowed to operate at the same time. For a software component to be included in a measurement it must be registered with the EMU, which is done via the user interface of the corresponding hardware component. Once registered, a component waits for a signal from the EMU that tells it to start. The EMU has a list of all the registered software components. Components that are not registered can still be operated, but will not be included in the automated measurement.

Figure 2.3 shows a schematic representation of the system. The dashed rectangles define autonomous software components and user interfaces, used to drive specific hardware. Each user interface enables the control of a hardware component, independent from all other hardware. In addition hardware components can be used together by registering the components with the experimental marshal unit (EMU). Using this approach enables simple addition and removal of hardware.

During combined experiments the manager software generate an XML-based output file that contains all relevant experimental settings, measured data, and links to files like measured spectra and pictures. Therefore, the file manager keeps a complete measurement tree in memory to quickly modify both the XML header information and the measurement steps. If an experiment crashes only the last measurement is lost. This XML file should be seen as a logbook and is needed for reference when the results are studied.

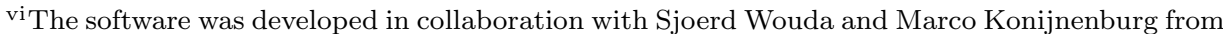
the Software Engineering department of the FOM Institute AMOLF, where the programming work took place.
} 


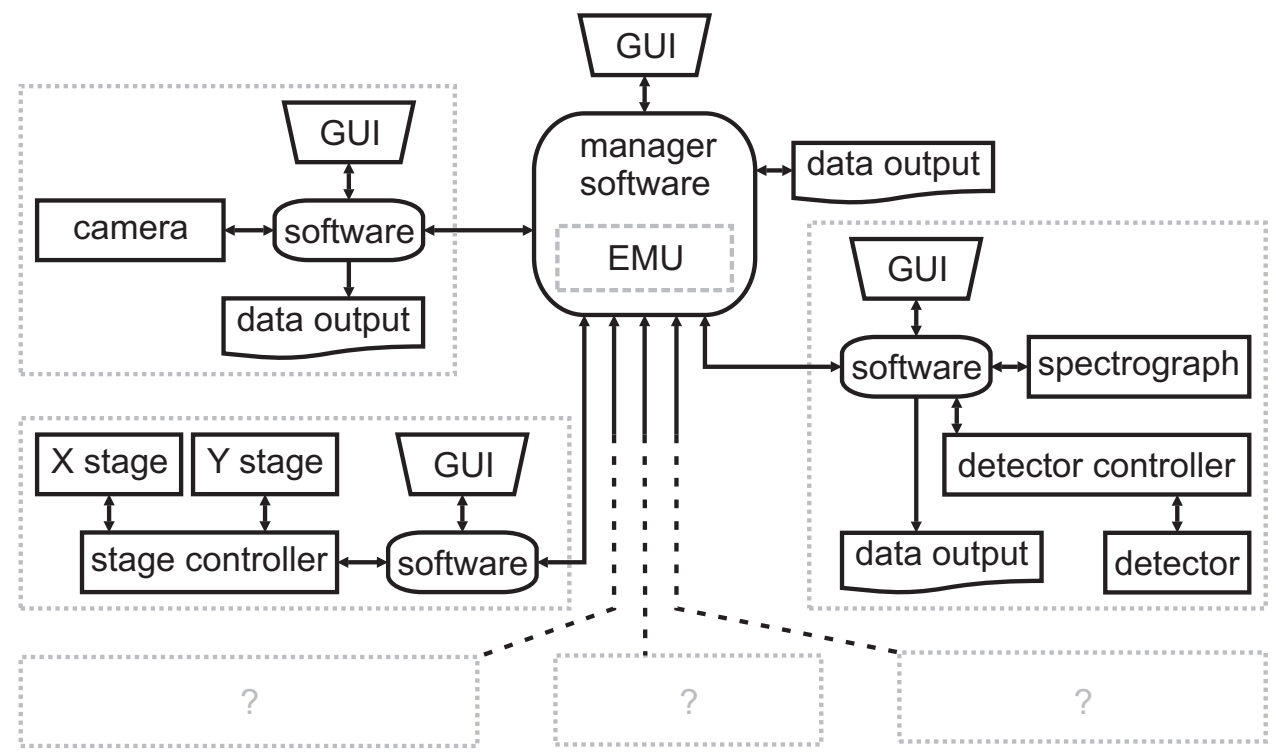

Figure 2.3: Illustration of the hardware control. Dotted rectangles enclose all aspects belonging to a single hardware component. Each dotted rectangle can be operated individually. Graphical user interfaces (GUIs) command the software that controls the hardware components. Each component can be linked to the manager software for automated experiments. This manager contains the experimental marshal unit (EMU) that determines which component is allowed to do something. Data output files can be generated by different software components. For clarity not all available hardware is presented. Dotted rectangles with question marks represent components that can easily be added in the future.

Most of the software is written in LabVIEW (version 8.21) with some parts in C\# (.Net 2.0). The developed software calls to vendor software that is provided with the hardware. Measurement results are stored in the format given by the vendor software. Each measurement yields a different file, which are all addressed in the XML output file of the manager. This way it is possible to look at measurement files with first results while the experiment is still running.

\subsection{Performance and discussion}

To determine if the setup works according to the intended specifications (Table 2.1) several test were done as demonstrations of the potential of the setup. As a guide, the components of Table 2.1 are used for the following discussion, starting with the microscope part. Figure 2.4 considers the alignment stages. All stages were tested in a Michelson interferometer setup, where the stages are used to move the mirror 

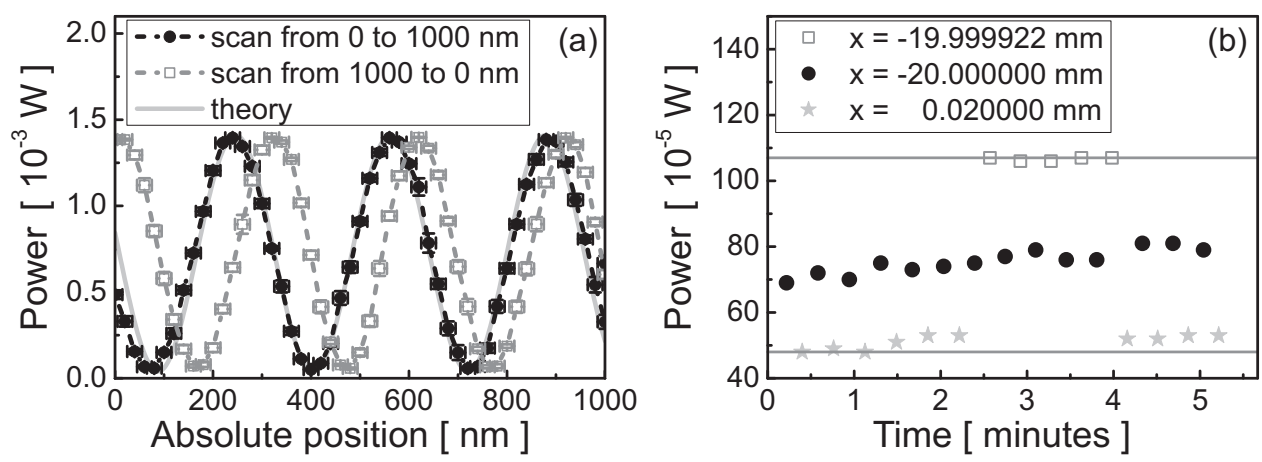

Figure 2.4: Stage test results using an interferometer configuration, with stages used to translate the cryostat. (a) Michelson interference signal versus absolute position of a scanning mirror on the XMS50 stage. Symbols denote measurements. Black dots show the first scan from position 0 to $1000 \mathrm{~nm}$. Open squares show the scan back from position 1000 to $0 \mathrm{~nm}$. Light gray line shows the expected behavior. Temperature changes during the experiment are believed to cause the difference between the two sets of data. (b) Interference signal measured at separately fixed positions versus time. Similar setup as in (a) but with XMS160 stage. Horizontal lines denote the maximum and minimum interference signal. The distance difference for the open squares and black dots correspond to 1/4 period of the interference signal. Hence, the signal jumps from the interference maximum to halfway. The signals are clearly separated for the different positions.

in the scanning arm. A helium-neon laser was used as light source. Figure 2.4.(a) shows a manual scan from absolute stage position 0 to $1000 \mathrm{~nm}$, which takes about 40 minutes. One hour later the stage is moved back from 1000 to $0 \mathrm{~nm}$. Clearly the positions differ by about $70 \mathrm{~nm}$, which is smaller than the requested $100 \mathrm{~nm}$ bi-directional repeatability. The measured period differs maximally $5 \%$ from the theoretical period of half the laser wavelength, i.e., $316.4 \mathrm{~nm}$. Figure 2.4.(b) shows the result of an experiment on another stage. The position of the stage is made to jog between predefined absolute values spaced by no less than $20 \mathrm{~mm}$. Still the symbols are clearly separated. The power only drifts slightly at position $\mathrm{x}=$ $-20.000000 \mathrm{~mm}$, where the signal is most sensitive to changes in the position. The stages are equipped with absolute encoders and the drift of the position is therefore believed to be caused by temperature differences in the setup. If a distance of $20 \mathrm{~mm}$ is considered and a linear thermal expansion coefficient of $\alpha_{\mathrm{L}}=11 \cdot 10^{-6} \mathrm{~K}^{-1}$ for the grade 430 stainless steel table-top is used, the $\Delta L=70 \mathrm{~nm}$ can already be explained by a temperature change $\Delta T=\Delta L / \alpha_{\mathrm{L}} L=0.32 \mathrm{~K}$, less than the typical temperature stability in the lab (see Figure 2.7.(a)). Hence, we conclude that the stages work to specifications and the importance of temperature fluctuations are relevant. 

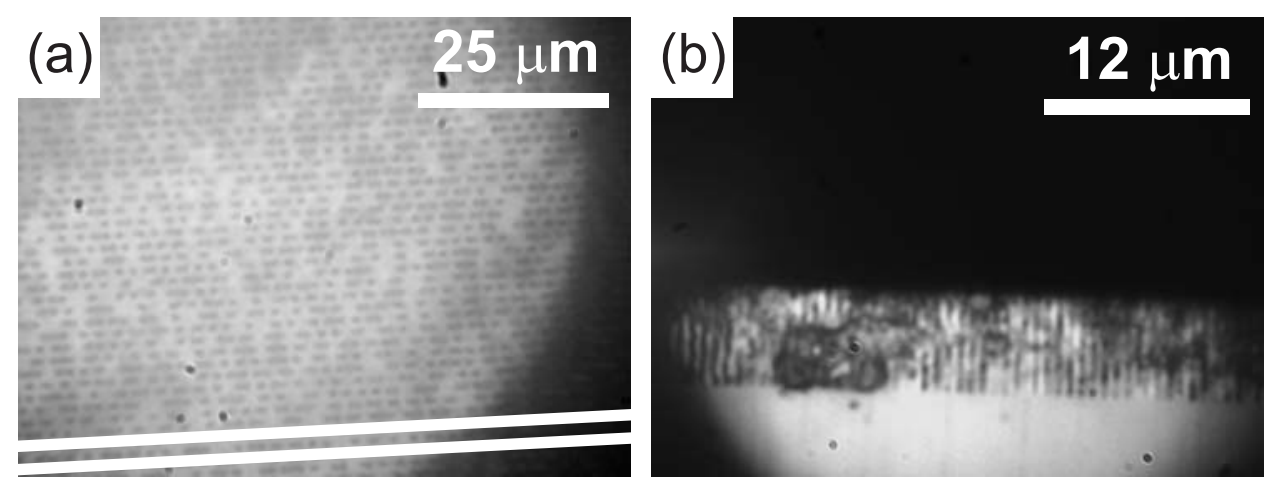

Figure 2.5: Images taken with the CCD camera. (a) Compact disc surface. The tracks that consist of the interrupted, dark stripes are clearly visible. Parallel white lines overlap with two different tracks, as a guide to the eye. (b) $2 D$ photonic crystal made of vertically aligned, etched pores on the edge of a silicon wafer. The pitch of the pores is $0.69 \pm 0.02 \mu \mathrm{m}$. Both graphs come with a different scale bar. The dark, curved shadow is the back aperture of the objective. The graphs show that the white light illumination of the sample is adequate. Samples can therefore be aligned for experiments, using this camera. Furthermore the spatial resolution is in agreement with the posed requirement.

Figure 2.5 shows two images taken by the camera in the setup, using different combinations of objective and camera lens (see also Figure 4.4). Figure 2.5.(a) shows the surface of a compact disc. The tracks, which are separated by $1.6 \mu \mathrm{m}$ are clearly resolved. Figure 2.5.(b) shows the side view of a two dimensional silicon photonic crystal that consists of $5.8 \pm 0.1 \mu \mathrm{m}$ deep pores with diameters of $0.36 \pm 0.02 \mu \mathrm{m}$ with a pitch of $0.69 \pm 0.02 \mu \mathrm{m}$ [92]. Clearly separated pores are recognized as vertical lines. Therefore the required $<1 \mu \mathrm{m}$ spatial resolution has been achieved, and it is even better than $0.69 \mu \mathrm{m}$.

Different light sources were used to test the spectrometer. Figure 2.6.(a) shows four different measurements to assess the available bandwidth. The left part of (a) shows light collected from the helium-neon laser, using the two different gratings that are available on the turret in the spectrograph. A sharp peak appears at the expected wavelength $\lambda=632.8 \mathrm{~nm}$. The right part shows light measured from the supercontinuum white light source (Fianium), together with a measurement where all light sources are off. The spectrum looks similar to the one provided by the manufacturer, with the sharp peak at $\lambda=1064 \mathrm{~nm}$, from the main oscillator inside the Fianium. The fringes around $\lambda=1400 \mathrm{~nm}$ are also observed in quantum dot emission spectra measured with this spectrometer in different experimental setups, see for example Figure 4.7, and Figure 4.8. These fringes are therefore attributed to the spectrometer. The detector's sensitivity vanishes above $\lambda=1650 \mathrm{~nm}$ (not reached for the grating position used here), whereas a cutoff at $\lambda=2000 \mathrm{~nm}$ was 

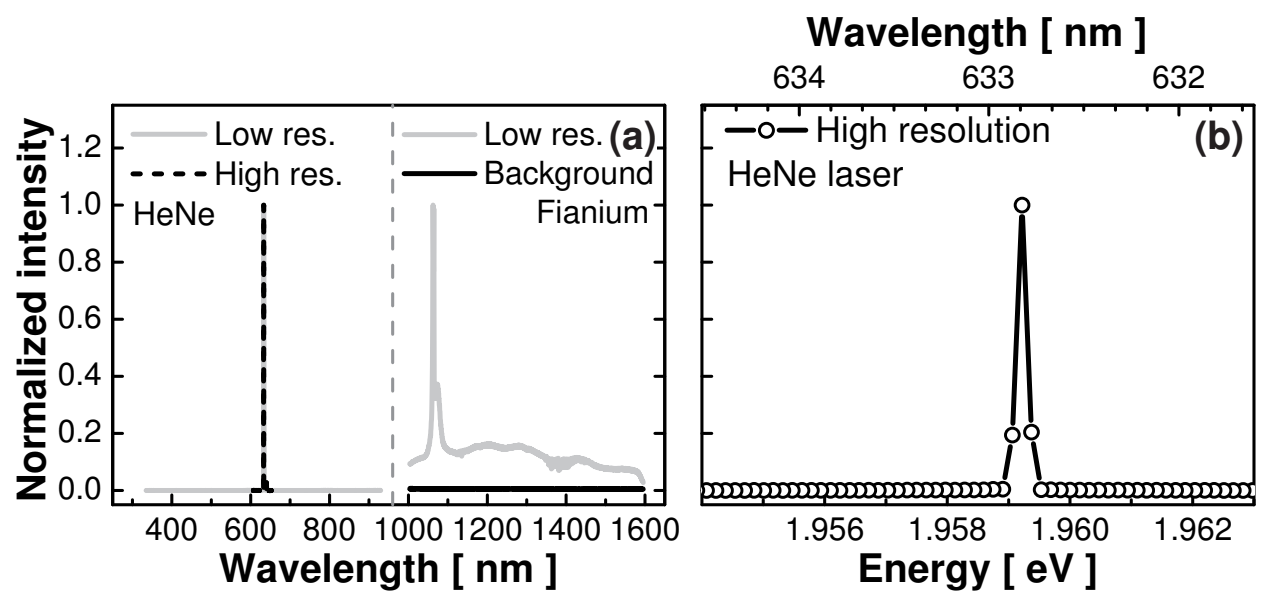

Figure 2.6: (a) Left part: measured helium-neon laser spectrum, using the low resolution (gray line) and high resolution (black dashed line) grating in the setup. The bandwidth of this experiment is $\Delta \omega / \omega=0.94$. Right part: measured spectrum of Fianium white light source (gray line) and a background measurement without light (black line). The background spectrum is flat, whereas the Fianium signal shows structure with a very pronounced peak at $\lambda \approx 1064 \mathrm{~nm}$. Fringes at $\lambda=$ $1400 \mathrm{~nm}$ are caused by the spectrometer. (b) Zoom in on the high resolution heliumneon data from (a). Symbols show the measurement values of the detector pixels and are connected through a line to guide the eye. The data are clearly centered at the expected wavelength of $\lambda=632.8 \mathrm{~nm}$. The relative distance between two pixels is $\Delta \omega / \omega=0.80 \cdot 10^{-4}$ for the high resolution measurement. All data sets were normalized to their maximum.

intended. For the upcoming experiments there is no need for additional detectors to probe the wavelength range from $1650<\lambda<2000 \mathrm{~nm}$. However, the design of this setup allows for addition of supplementary hardware, as the additional design rule to make the setup suitable for changes was considered with care. From the heliumneon measurement the full energy range per central energy is calculated which gives the relative bandwidth of the measurement $\Delta \omega / \omega=0.94$, which is more than three times larger than the requirement in Table 2.1.

Figure 2.6.(b) shows a zoom in of the helium-neon data from Figure 2.6.(a) to assess the resolution. The high resolution grating yields the laser line exactly at the right energy. A relative energy difference between two pixels is $\Delta \omega / \omega=0.80 \cdot 10^{-4}$, which is $20 \%$ better than required spectral resolution. In practice the resolution is given by the relative difference over three pixels, which results in a spectral resolution of $\Delta \omega / \omega=2.4 \cdot 10^{-4}$. All spectra were recorded with an InGaAs diodearray detector, that has pixel widths of $25 \mu \mathrm{m}$. Even higher spectral resolutions can be obtained for the time-resolved measurements with the photomultiplier detector, 

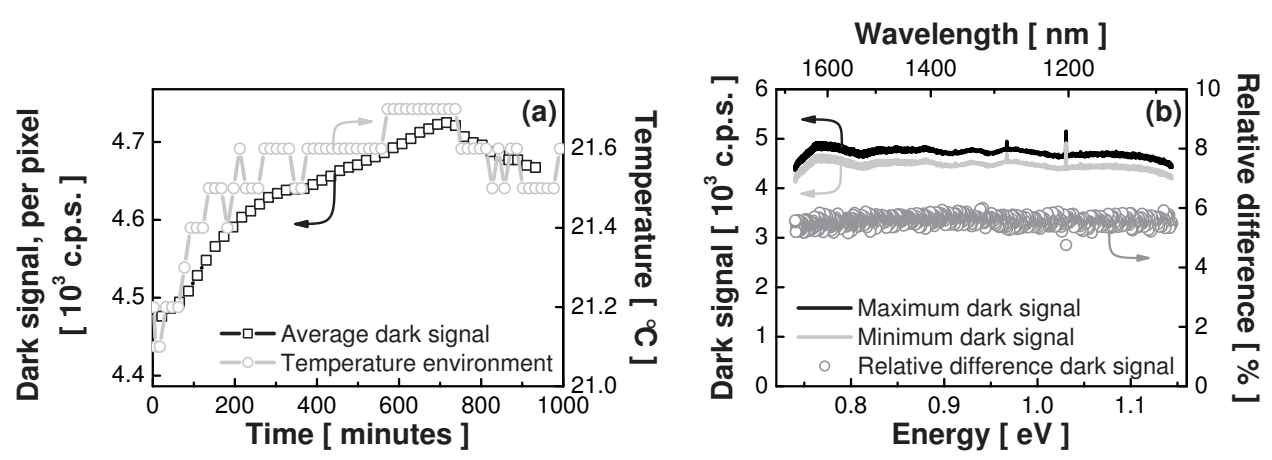

Figure 2.7: (a) Time-dependent average dark signal of the InGaAs array detector, used to measure spectra at near-infrared wavelengths (left ordinate). The timedependent temperature of the detectors' surroundings is plotted (right ordinate). Both curves show the same time dependence. $0.1^{\circ} \mathrm{C}$ jumps in the temperature result from the resolution of the thermometer. (b) Dark signal spectra that correspond to the maximum and minimum signal from (a) (left axes), together with their relative difference (right axes). The dark signal varies uniformly over the complete spectral range. All symbols denote the measured data.

by changing the width of the exit slit of the spectrograph to less than $25 \mu \mathrm{m}$. This was successfully tested (not shown). The interchangeable grating turret provides the means to obtain even higher spectral resolution if such might be required in future experiments.

The detector to measure spectra clearly works. In addition a fast detector was needed for time-resolved emission measurements. A photomultiplier module is used because this enables the measurement of emission intensity decay curves over a large time delay. InGaAs avalanche photodiodes (APDs) were also considered but rejected because the limited gate time (100 ns) makes their use very impractical. The photomultiplier used limits the time resolution of the setup to an experimentally determined $288 \pm 2 \mathrm{ps}$, see Section 5.2.1. This time resolution is 3.5 times faster than the required nanosecond resolution.

Figure 2.7.(a) shows the temporal change in dark signal, measured with the InGaAs line detector. At the same time the temperature in the environment is monitored. The increase and decrease of the dark signal closely correlate with the changes in the temperature of the surroundings. The jumps in the temperature data are caused by the $0.1{ }^{\circ} \mathrm{C}$ resolution of the digital thermometer. The dark signal increases fastest between $0<$ Time $<200$ minutes, with a slope of about $0.4 / 200$ ${ }^{\circ} \mathrm{Cmin}^{-1}$. Hence, to obtain less than $1 \%$ difference in the background signal, this measurement should be repeated within one hour. Figure 2.7.(b) shows the raw data of the maximum and minimum background signal in combination with their relative difference. The spectra are vertically offset by about $5.5 \%$ with respect 

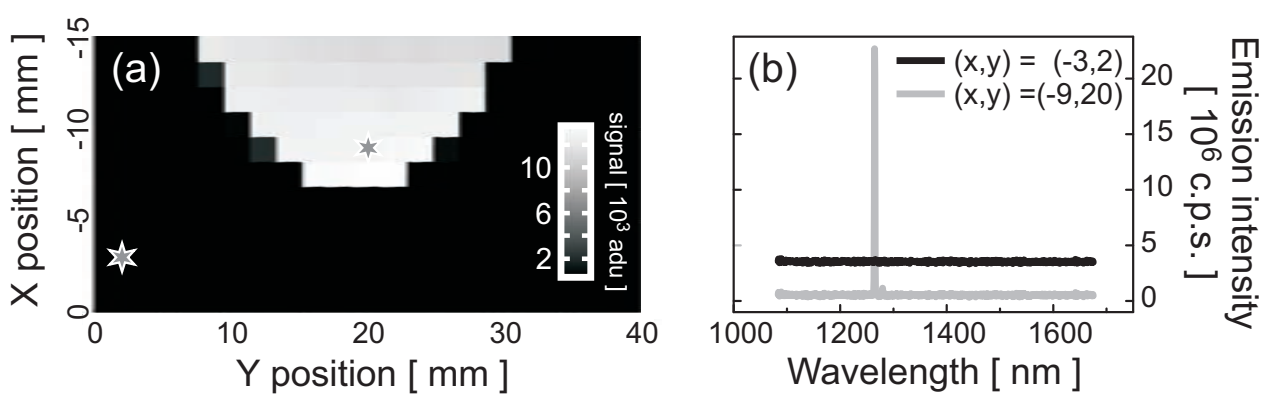

Figure 2.8: (a) Reflectance scan over part of a gold mirror. White area corresponds to the mirror, dark area is besides the mirror. Color scale denotes the raw output signal from the detector, and the two stars mark the positions used for (b). (b) Two spectra measured in (a), one besides the mirror at coordinates $x, y=-3,2$ and one on the mirror at $x, y=-9,20$. The first measurement is translated vertically by $3 \cdot 10^{6}$ c.p.s. for clarity.

to one another, which is larger than the $2.5 \%$ response non-linearity specified for this detector. During these measurements the humidity around the detector was also monitored (not shown). The relative humidity increase from $28 \%$ to $39 \%$ does not follow the same temporal dependence found for the temperature and the background signal. Hence, only the temperature is important for the dark signal.

The detectors are specified in Appendix A and need no further demonstration here. Also, the high magnification objective that combines high NA with long working distances, was selected with the implementation of a microscope cryostat in mind.

As a final demonstration Figure 2.8.(a) shows an area scan made over part of a 1" mirror, using the helium-neon laser. A combination of two stages and the spectrometer was used to collect light reflected from the mirror and store the spectra separately for each set of X,Y coordinates. Clearly, the mirror stands out of the background because of the high reflected signal. In this figure, only the spectral intensities measured at the laser peak frequency are used. The figure was automatically generated using developed software that uses the XML output file to find, read and process measurement data-files. Figure 2.8.(b) shows the complete spectra recorded at two positions, one on the mirror, and one besides the mirror. The spectrometers' output signal units (analog-to-digital units, or ADU) are converted to photon counts per second. On the mirror, the second order grating mode of the helium-neon laser appears clearly at $\lambda=1265.6 \mathrm{~nm}$. Besides the signal reflected from the mirror, the spectrum is determined by the background signal and readout noise. This example shows the manager software works and the setup can be used to automatically run experiments in which all hardware components can be combined. 
Two parts of the planned setup have not been implemented to date, i.e., the cryostat to control the temperature, and the Hanbury Brown and Twiss part for antibunching experiments. It should be mentioned that so-called microscope heliumflow cryostats are available that match the temperature requirements, and are also light enough to be moved with the intended stage-combination. Although these components are required for the intended single emitter experiments, they are not necessary for the work described in this thesis. Due to the modular design of the software, these components can be implemented without the need to change the operational software. Only additional software is needed to control the hardware and register the component to the existing manager software for automated measurements.

\subsection{Conclusions}

The experimental setup needed for spontaneous emission control in nanophotonic samples had been described. As demonstrated, the positioning, spatial, and spectral resolution meet the specifications. We determined a spatial resolution better than $0.69 \mu \mathrm{m}$, and a positioning accuracy of $70 \mathrm{~nm}$. Broadband measurements over a relative frequency range of $\Delta \omega / \omega=0.94$ are possible and spectral resolutions of $\Delta \omega / \omega=2.4 \cdot 10^{-4}$ were achieved using the spectral change over three pixels. Furthermore, the timing resolution for time-correlated single photon counting experiments is 288 ps. The cryostat and APD detectors have not been implemented at this time. However, it was shown that the modular design of the software enables straightforward addition of these components, and the installed hardware is selected with the additional components in mind. Currently, the setup allows for spatially, spectrally and temporally resolved measurements over a large wavelength range from 400 to $1650 \mathrm{~nm}$. These measurements are needed to study the emission of light sources inside photonic crystals at near-infrared wavelengths, which is the content of Chapters 4 to 6 . 



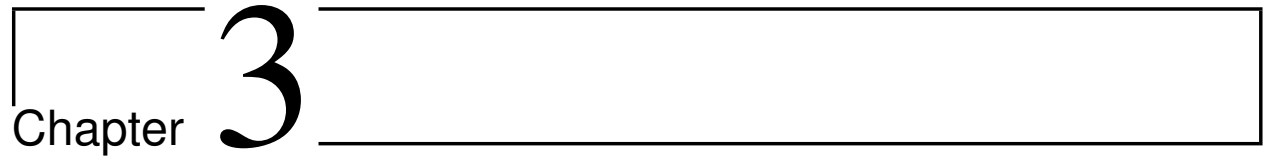

\section{Properties of PbSe quantum dots}

\subsection{Introduction}

Quantum dots are tiny particles that consist of hundreds to many thousands of atoms [65]. In this thesis the focus is on semiconductor quantum dots, or nanocrystals. In bulk semiconductors conduction electrons, and holes form exciton polaritons which are free to move around in the solid. Therefore, the exciton energy spectrum is almost continuous. For small semiconductor sizes confinement effects start to influence this exciton spectrum [93, 94]. These confinement effects become pronounced when the crystallites' radius is smaller than the Bohr radius of the exciton $[67,94-100]$. As a result the low energy intraband transition of these quantum dots becomes discrete and size dependent. This results in discrete, tunable emission lines.

Semiconductor quantum dots form a very interesting class of light emitters, because: (1) the dots exhibit a discrete energy spectrum, (2) the energy spectrum, photoluminescence emission wavelength and radiative lifetime can be controlled via the size of the quantum dots [97, 101], (3) the dots display high quantum efficiencies, even at near-infrared wavelengths [98, 100-102], and (4) the dots do not bleach, in contrast to dye molecules.

The discrete energy spectrum distinguishes quantum dots from other solid-state systems and has caused them to be called artificial atoms. The atom-like properties make them suitable for studying the physics of confined carriers and manybody effects [65, 103-109]. The dots could also lead to novel device applications in fields such as quantum cryptography, quantum computing, optics, pressure sensing, telecommunication, and optoelectronics [110-115]. Furthermore, quantum dots are attractive materials for bio-labeling [116-120], solid state lighting [121-123], optical amplification and lasing [101, 124-129].

We are interested in near-infrared emission, and use colloidal PbSe quantum dot 
ensembles as light source. In these IV-VI semiconductor quantum dots both the electron and the hole are strongly confined. This is in contrast to most II-VI (like CdSe), and III-V material quantum dots where only the electron is confined, as the Bohr radii of the heavy holes are typically smaller than the quantum dot radius [67]. The exciton Bohr radius ${ }^{\mathrm{i}}$ in $\mathrm{PbSe}$ is $a_{\mathrm{B}}=46 \mathrm{~nm}$ which is much larger than the typical PbSe quantum dot radius of $r=2.4 \mathrm{~nm}$. For comparison, the exciton Bohr radius in CdSe is $a_{\mathrm{B}}=6 \mathrm{~nm}$ with quantum dot radii of typically $r=2 \mathrm{~nm}$ [131]. As a consequence of the strong confinement the exciton-phonon interaction that causes spectral line-broadening is reduced. Hence, the absorption and emission spectra of PbSe quantum dots are relatively simple and sparse [67, 132]. Furthermore, the strong confinement in small $\mathrm{PbSe}$ quantum dots results in a weak temperature dependance of the spectrum and decay rate, expected for atom-like levels, but not observed in other semiconductors [132, 133].

In this chapter, the quantum dots used throughout this thesis are characterized. Physical properties of the dots are derived from measurements. First, the fabrication and luminescent efficiency of these dots are discussed (Section 3.2). Subsequently, a theoretical derivation is given to find the relation between excitation conditions and the number of excitons created in a quantum dot (Section 3.3). Then, the absorption cross section and oscillator strengths are considered by means of absorption and emission measurements (Section 3.4). Hereafter, dynamical properties are derived from time-resolved emission experiments and a measure is found for the inhomogeneous broadening of the emission spectrum (Section 3.5). The chapter ends with conclusions and remarks (Section 3.6).

\subsection{Fabrication and efficiency of quantum dots}

The synthesis and characterization of monodisperse PbSe quantum dots was first realized in a glass host material [134]. Later, a more versatile liquid-phase synthesis of spherical colloidal dots was invented and adapted [68, 135-137]. Figure 3.1.(a) shows a transmission electron microscope (TEM) image of a single PbSe quantum dot. A regular interference pattern is observed at the location of the quantum dot, due to the layered ordering of the $\mathrm{Pb}$ and $\mathrm{Se}$ atoms in this nanocrystal. PbSe quantum dots crystallize in a cubic sodium chloride (rock salt) structure [137]. In Figure 3.1.(b) the ordering of the atoms in the rock salt structure is shown [102]. Clearly, the calculation shows the layered structure of $\mathrm{Pb}$ and Se planes that form the quantum dot. These layers appear as dark and bright stripes in the TEM image.

Although there is consensus about the physical structure of the dots there are many optical properties that need clarification. A very important property of light emitters is the luminescence quantum efficiency $\eta$. In general $\eta$ is given by the ratio of the number of emitted photons per excited exciton, often given as a percentage ${ }^{i i}$.

\footnotetext{
${ }^{\mathrm{i} E x c i t o n}$ Bohr radius: $a_{\mathrm{B}}=\epsilon \hbar^{2} / 2 m^{\star} e$, with $\hbar$ the reduced Planck constant, $\epsilon$ the dielectric constant of the semiconductor, $e$ the charge, and $m^{\star}$ the effective mass of the electron and hole [130]

${ }^{\mathrm{ii}}$ Using this definition it is possible to obtain quantum efficiencies larger than one, which might
} 

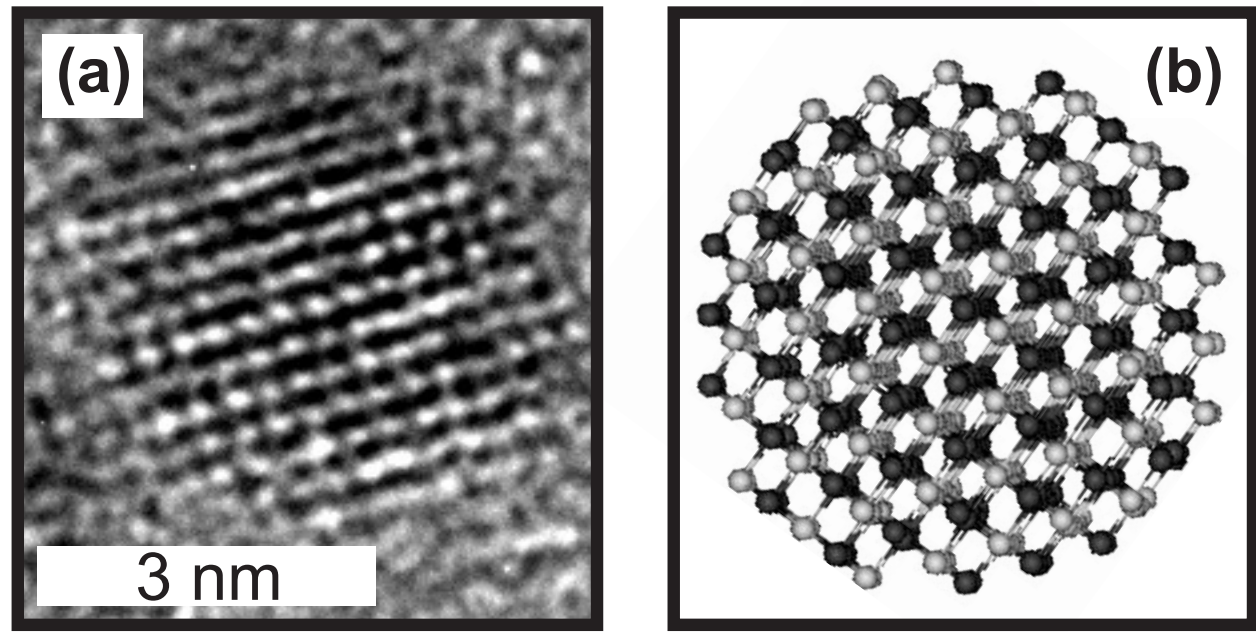

Figure 3.1: Structure of colloidal PbSe quantum dots. (a) Transmission Electron Microscope (TEM) image of a single PbSe quantum dots (by Niels Zijlstra). The scale bar in the lower left corner is $3 \mathrm{~nm}$. (b) Rock-salt crystal structure of PbSe quantum dot as derived from a density functional calculation, reproduced from [102]. This dot has a radius of $1.48 \mathrm{~nm}$. The dark and light spheres represent the $\mathrm{Pb}$ and Se atoms respectively. This figure is rotated such that the quantum dots' orientation is the same as (a). The dark and light stripes in the TEM image of (a) correspond to the $\mathrm{Pb}$ and Se planes in (b). Both figures are reproduced with permission from the authors.

For $\mathrm{PbSe}$ dots quantum efficiencies have been reported ranging from $\eta=12 \%$ to $\eta=89 \%[68,101,111,129,136,143,144] . \quad \eta$ may change if the quantum dots are placed in a different environment, as different environments may entail different non-radiative and radiative decay rates. The absence of dangling bonds at the surface is believed to cause both (1) the high quantum efficiencies in spite of a long lifetime, and (2) the small Stokes shifts $[68,136]$. Also, the simpler bandedge structure (as compared to, e.g., CdSe quantum dots) is likely to improve the fluorescence efficiency [68]. In contrast to other dots, the PbSe dots do not seem to blink, or have surface trapped states [98, 102].

Although the quantum efficiency of our dots has not been determined directly, the influence of the local radiative density of states (LDOS) on the total decay rate is demonstrated in Chapter 6. As only the dots' radiative decay rate is influenced by the LDOS the observed change in the total decay rate (i.e., radiative plus nonradiative rate) denotes a quantum efficiency larger than $30 \%$.

result from carrier multiplication [138-142]. 

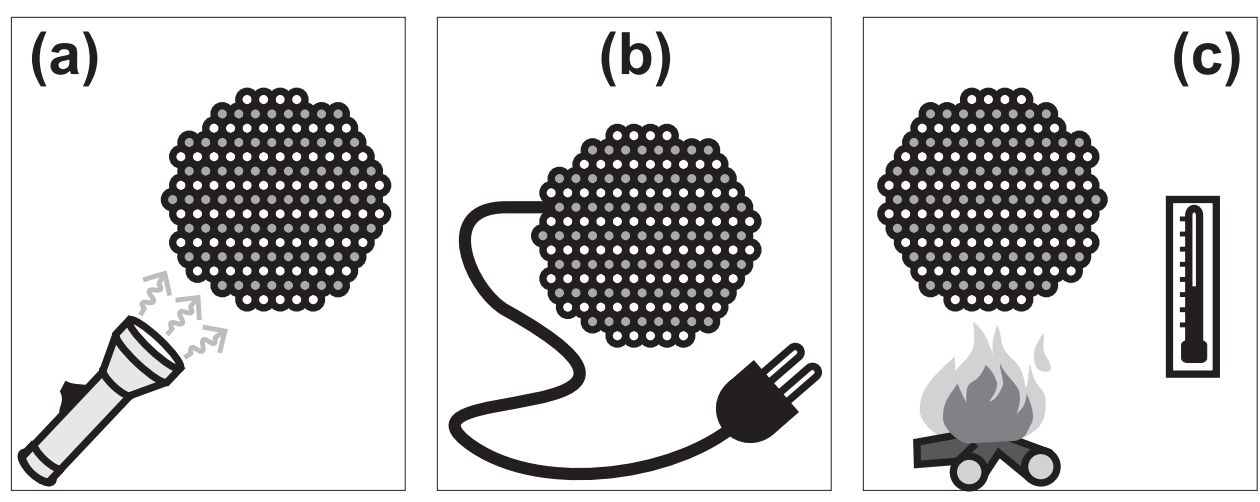

Figure 3.2: Cartoon of most common ways to excite quantum dots. (a) Optically, using an excitation light source like a laser, (b) electrically, and (c) thermally. In each figure the quantum dot is illustrated by the circular lump of spheres. Throughout this thesis, only optical excitation is used.

\subsection{Excitation of single excitons in quantum dots}

\subsubsection{Overview}

To interpret the quantum dots' emission spectrum and decay dynamics the excitation conditions should be understood. There are several possibilities to excite quantum dots. Figure 3.2 shows the most commonly used ways: (a) optically, (b) electrically, and (c) thermally. In Chapters 5 and 6 pulsed, optical excitation is needed. Therefore, pulsed excitation is assumed for the remainder of this chapter. More specifically, a pulsed laser is used to excite the quantum dot, thereby creating one or more exciton polaritons ${ }^{\mathrm{iii}}$, inside the dot. Multiple-excitons are not wanted, as they disturb our simple two level system picture, and may quench the radiation decay due to Auger recombination processes ${ }^{\mathrm{iv}}$. With the excitation intensity and the excitation wavelength it is possible to assess the excited state of the quantum dot. Consequently the chance that multiple excitons are created can be evaluated, which is shown below.

It is important to note that PbSe quantum dots are known for their highlyefficient multiple exciton generation that result from the Coulomb interaction between independent electrons and holes [146]. This means that a single photon absorption may result in multiple excitons. For PbSe quantum dots this effect be-

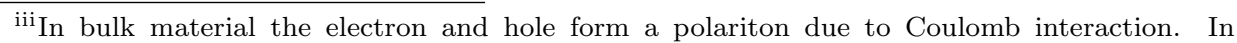
contrast, the confinement energy that originates from the small quantum dot size keeps the electron and hole together in PbSe quantum dots. The Coulomb energy is negligible compared to this confinement energy. Still the term exciton polariton is used in quantum dots.

${ }^{i v}$ Note that multi-exciton generation is a much faster process than Auger recombination [141, 145].
} 
comes large if the excitation photon energy exceeds three times the energy of the quantum dots' band gap [140, 142, 147-149]. However, a slight increase in the quantum yield was already observed for photon energies between two and three times the band gap energy $[138,150]$. Pseudopotential calculations have revealed a threshold for multi-exciton generation at about 2.2 times the band gap energy [141]. The largest photon energy used throughout this thesis is $2.3 \mathrm{eV}$ (i.e., $532 \mathrm{~nm}$ light). The absorption peak of the dots lies at $0.98 \mathrm{eV}$, as determined in Section 3.4. Therefore, the excitation photon has an energy of 2.3 times the quantum dots' band gap. Hence, there is a small chance for multiple exciton generation. However, at these energies, we have performed transient absorption measurements that revealed negligible effects on the absorption. Furthermore, the absorption measurements revealed that multi-excitons decay within a few hundred picosecond, which is three orders of magnitude faster than the radiative lifetime of these quantum dots [138, 140, 149]. Hence, the influence of multi-exciton generation can be neglected in our decay curve measurements (Chapters 5 and 6).

Another route to multi-exciton generation is the use of large excitation intensities. As explained in Appendix B, the chance $P$ for a single excitation pulse to create a certain number of excitons $n_{x}$, can be approximated by the Poisson distribution, hence,

$$
P\left(n_{x}\right)=\frac{(N p)^{n_{x}} e^{-N p}}{n_{x} !},
$$

where $N$ is the number of photons in the excitation pulse, and $p$ is the chance for a single photon to create an exciton. The mean number of excitations $\left\langle\mathrm{n}_{\mathrm{x}}\right\rangle$ created by a single pulse is subsequently derived and results in

$$
\left\langle\mathrm{n}_{\mathrm{x}}\right\rangle=\frac{E}{\epsilon} \cdot \frac{8 C_{\mathrm{abs}}(\epsilon) \mathrm{NA}^{2}}{\pi \lambda_{0}^{2}} .
$$

Here $E$ is the pulse energy, $\epsilon$ is the single-photon energy, $C_{\text {abs }}$ is the absorption cross-section of the emitter, NA is the numerical aperture of the focusing lens and $\lambda_{0}$ the wavelength in vacuum. This simple expression serves as an important tool during experiments. We are now able to find sound experimental conditions, such that multiple exciton generation can be ruled out. These experimental conditions are the topic of the following section.

\subsubsection{Determination experimental excitation conditions}

In this section Equations 3.1 and 3.2 are used to find experimental conditions that prevent multiple exciton creation in single quantum dots. For the experiments in Chapters 5 and 6 practical issues require a long (typ. $>3 \mathrm{~mm}$ ) working distance objective. Therefore, a microscope objective with a numerical aperture NA $=0.05$ is used. The wavelength of the excitation pulses is $\lambda_{0}=532 \mathrm{~nm}$, which corresponds to a photon energy $\epsilon=3.73 \cdot 10^{-19} \mathrm{~J}(=2.3 \mathrm{eV})$. For this photon energy $C_{\text {abs }}(\epsilon)$ can be estimated by linear extrapolation of the small dot results from Figure 3.4 below, 


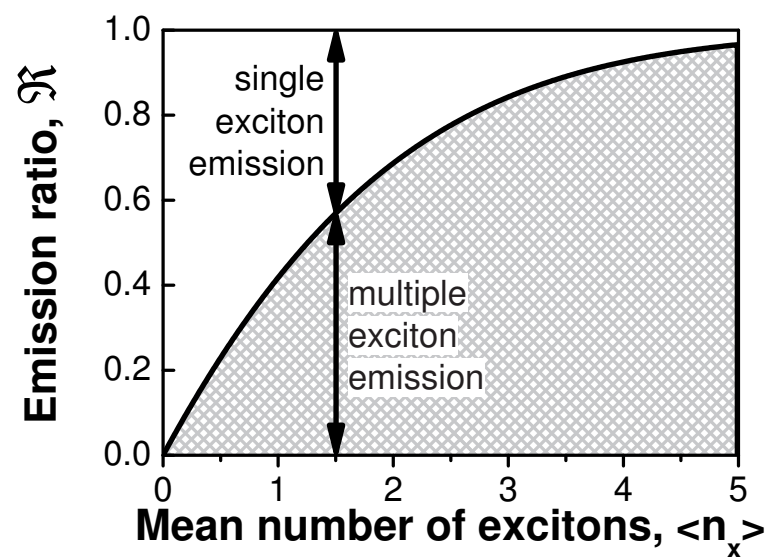

Figure 3.3: Contribution of multiply excited quantum dots to emission intensities, as a function of $\left\langle\mathrm{n}_{\mathrm{x}}\right\rangle$, the average number of excitons created in a single quantum dot. Hatched region is the part that stems from multiply excited dots. White region stems from dots that only contain one exciton. The black arrows are added to illustrate the ratio at a given $\left\langle\mathrm{n}_{\mathrm{x}}\right\rangle$.

which yields $C_{\text {abs }}(532.8 \mathrm{~nm})=1.3 \cdot 10^{-19} \mathrm{~m}^{2}$. Typically, excitation powers of $5.0 \mu \mathrm{W}$ are used at a laser pulse repetition rate of $203 \mathrm{kHz}$. Hence, the energy per pulse is $E=5.0 \cdot 10^{-6} / 203 \cdot 10^{3}=2.5 \cdot 10^{-11}[\mathrm{~J}]$. Substitution of these preceding parameters into Equation 3.2 yields $\left\langle\mathrm{n}_{\mathrm{x}}\right\rangle=0.19$. Hence, a single laser pulse creates on average 0.19 excitons in a quantum dot at the center of the laser focus. The chance $P\left(n_{x}\right)$ to create a certain number $n_{x}$ of excitons can be calculated using Equation 3.1. For the given parameters this yields $P(0)=0.82, P(1)=0.16$, and $P(2)=0.015$. Hence, the chance to create more than one exciton is $P\left(n_{x}>1\right)=1-P(0)-P(1)=0.016$, which is ten times smaller than the chance to create a single exciton.

With use of Equation 3.1, it is possible to calculate the ratio between the chance to create multiple excitons in a quantum dot and the chance to create one or more excitons ${ }^{\mathrm{v}}$. This yields

$$
\Re\left(\left\langle\mathrm{n}_{\mathrm{x}}\right\rangle\right)=\frac{P\left(n_{x}>1\right)}{P\left(n_{x}>0\right)}=\frac{1-P(0)-P(1)}{1-P(0)}=1-\frac{N p}{e^{N p}-1}=1-\frac{\left\langle\mathrm{n}_{\mathrm{x}}\right\rangle}{e^{\left\langle\mathrm{n}_{\mathrm{x}}\right\rangle}-1} .
$$

Assume the quantum efficiency not to change with the number of excitons created. As a consequence, the ratio $\Re\left(\left\langle\mathrm{n}_{\mathrm{x}}\right\rangle\right)$ corresponds to the contribution of the quantum dot's multiple exciton emission to the quantum dot's total emission, for a given $\left\langle\mathrm{n}_{\mathrm{x}}\right\rangle$. In Figure $3.3 \Re\left(\left\langle\mathrm{n}_{\mathrm{x}}\right\rangle\right)$ is plotted versus the mean number of excitons $\left\langle\mathrm{n}_{\mathrm{x}}\right\rangle$. The contribution of multi-exciton emission to the total emission intensity is

${ }^{\mathrm{v}} P(0)$ does not yield photons and its value is therefore not relevant, because the interest is in emission. However, $P(0)$ is used to calculate the ratio between $P\left(n_{x}>1\right)$ and $P\left(n_{x}>0\right)$. 
considerable, even for small $\left\langle\mathrm{n}_{\mathrm{x}}\right\rangle$. At the beginning of this section $\left\langle\mathrm{n}_{\mathrm{x}}\right\rangle=0.19$ was derived for the settings used in our experiments. For this case Equation 3.3 yields $\Re(0.19)=0.092$. Hence, on average $9.2 \%$ of the emission signal stems from multiple excitations in the quantum dot. This contribution is small but not negligible and therefore the excitation should remain low in this type of experiment. In the experiments throughout this thesis the emission signal is collected from ensembles of quantum dots, embedded inside titania inverse opal photonic crystals. Inside these strongly photonic samples the coherent beam will be attenuated. The excitation light diffusely scatters inside the sample which enables the excitation of quantum dots outside the coherent beam path. Furthermore, the quantum dot emission that escapes the samples is diffuse and, consequently, does not necessarily stem from a quantum dot that is positioned where the focus of the excitation beam is expected. Therefore, Equation 3.2 overestimates the average number of excitons $\left\langle\mathrm{n}_{\mathrm{x}}\right\rangle$ created in the quantum dots that contribute to our measured emission signal. As a consequence the contribution of multiply excited quantum dots to the emission signal is much smaller than $9.2 \%$. Therefore, the relations in Equations 3.2 and 3.3 yield the upper boundary for $\left\langle\mathrm{n}_{\mathrm{x}}\right\rangle$ and $\Re\left(\left\langle\mathrm{n}_{\mathrm{x}}\right\rangle\right)$. Keeping these equations in mind, we are able to find suitable excitation conditions. Indeed, further reduction of the energy per pulse does not yield a different decay dynamics, as shown in Section 5.2.2, which confirms that our experiments do not suffer from multiple excitons. Finally, it is mentioned that these equations are very general and can be applied to evaluate excitation conditions for other light sources as well.

\subsection{Spectral properties}

\subsubsection{Absorption measurements}

In this section absorption spectra of PbSe quantum dots are discussed. In addition, the energy dependent decadic molar extinction coefficient $\epsilon_{\text {ext }}(E)$, the absorption cross section $C_{\text {abs }}(E)$, and oscillator strengths $f$ are derived. First, coherent transmission measurements were done to determine the quantum dots' absorption spectrum. To derive $\epsilon_{\text {ext }}(E)$ from the absorption measurement the Beer-Lambert equation is used [151],

$$
\epsilon_{\mathrm{ext}}(E)=\frac{1}{c d} \log \frac{I_{0}(E)}{I(E)} .
$$

Here, $I(E)$ is the measured, coherent intensity transmitted through the quantum dot suspension with photon energy $E$, and $I_{0}(E)$ the energy dependent transmitted intensity through a reference sample without quantum dots. $c[\mathrm{M}]$ is the concentration of dots, and $d[\mathrm{~cm}]$ is the thickness of the sample. $\epsilon_{\mathrm{ext}}(E)$ is linearly dependent on the term $\log \left(I_{0}(E) / I(E)\right)$, which is also known as the absorbance or optical density $(O D)$. Hence, the peaks in $\epsilon_{\text {ext }}(E)$ correspond to the absorption peaks. The absorption cross section $C_{\text {abs }}(E)\left[\mathrm{cm}^{2}\right]$ is calculated using the Beer-Lambert equa- 


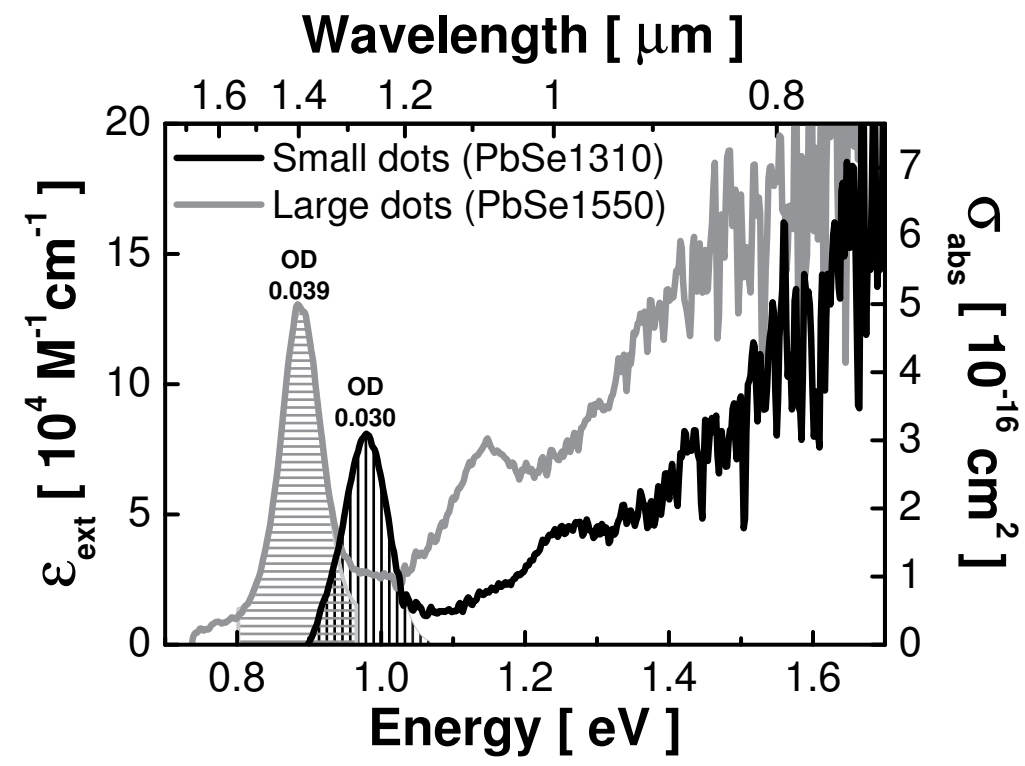

Figure 3.4: $P b S e$ quantum dot decadic extinction coefficients $\epsilon_{\mathrm{abs}}(E)$ and absorption cross-sections $C_{\text {abs }}(E)$ versus energy. The quantum dots used in both samples have different size distributions. The black (gray) line corresponds to the small (large) quantum dots. Both lines show clear peaks in the spectra, caused by confinement effects. The suspensions' optical densities (OD) at the energy of the first absorption peak are written above the peaks. The smaller dots reveal smaller absorption cross-sections. The rapid fluctuations at energies larger than $1.4 \mathrm{eV}$ are a result of the low signals collected in this range. The hatched areas show a single Gaussian fit, which will be used to calculate the oscillator strength. Between parenthesis are the quantum dots' brand names from the manufacturer, Evident.

tion,

$$
C_{\mathrm{abs}}(E)=\frac{1}{n d} \ln \frac{I_{0}(E)}{I(E)},
$$

where $n$ is the number of dots per cubic centimeter. From Equations 3.4 and 3.5 it can be derived that $C_{\text {abs }}(E)=3.82 \cdot 10^{-21} \epsilon_{\text {abs }}(E)\left[\mathrm{cm}^{2}\right]$.

Figure 3.4 shows the results for $\epsilon_{\text {ext }}(E)$ and the corresponding absorption crosssections $C_{\text {abs }}(E)$ measured from two suspensions of PbSe quantum dots in hexane. The quantum dots in both samples were bought from Evident Technologies and have different size distributions. Quantum dot diameters of approximately $4.7 \mathrm{~nm}$ $(\mathrm{PbSe1310})$ and $5.1 \mathrm{~nm}(\mathrm{PbSe1550)}$ were estimated from Evidents' specification sheets, whereas transmission electron microscopy measurements on the PbSe1550 dots indicate diameters that are about $25 \%$ smaller $^{\mathrm{vi}}$. Both graphs show the same

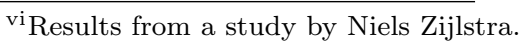


trend, with $\epsilon_{\text {ext }}(E)$ being zero at low energies and increasing for larger energies. Clear absorption peaks are observed. For the small dots these peaks are at $0.98 \mathrm{eV}$ and $1.27 \mathrm{eV}$ and for the large dots at $0.89 \mathrm{eV}$ and $1.15 \mathrm{eV}$. The peaks are due to the confinement effects discussed before. The first peak (low energy) corresponds to a $1 S_{h} \rightarrow 1 S_{e}$ inter-band transition. The origin of the second peak is still discussed in the literature. From experiment, effective mass calculations, and tight binding calculations it was deduced that the second transition is a $1 S_{h, e} 1 P_{e, h}$ transition $[98,152,153]$. However, this is in contrast to scanning-tunneling spectroscopy measurements, pseudopotential calculations, and pump-probe experiments where the second absorption peak is attributed to a $1 P_{h} \rightarrow 1 P_{e}$ inter-band transition [154-156]. The discussion on the second peaks' origin is of no concern for the study presented in this thesis, as only the first $1 S_{h} \rightarrow 1 S_{e}$ transition is relevant.

As expected, the smaller dots exhibit absorbance peaks at higher energies $[129$, 134-136, 153]. It has been demonstrated that the energy gap varies mainly as $1 / d$, for dots with the effective diameter $d$ in the range $2<d<20 \mathrm{~nm}[98,157]$. Note that the absorbance peak energies are about 2.5 times larger than the direct band gap energy of bulk PbSe, i.e., $0.37 \mathrm{eV}$ [158]. This difference is a measure for the degree of confinement, which is much larger in these lead salt IV-VI materials than in II-VI and III-V materials [67].

The absorption cross-sections of the $1 S_{h} \rightarrow 1 S_{e}$ transition reduces with size. This cross section is about $C_{1 S_{h} \rightarrow 1 S_{e}}=5.0 \cdot 10^{-16} \mathrm{~cm}^{2}$ for the large dots, and $C_{1 S_{h} \rightarrow 1 S_{e}}=3.1 \cdot 10^{-16} \mathrm{~cm}^{2}$ for the small dots. Ergo, the absorption cross-section is almost three orders of magnitude smaller than the geometrical cross-section of the quantum dot, which is about $2 \cdot 10^{-13} \mathrm{~cm}^{2}$, and within the Rayleigh-Gans regime. These absorption cross sections are in accordance with values between $10^{-16}$ to $10^{-15} \mathrm{~cm}^{2}$, mentioned earlier for PbSe dots [129], and thereby similar to cross sections of other colloidal quantum dots that emit in the visible to near-infrared regime. For comparison, the transition cross section of erbium atoms, often used as the emitter of choice for telecom applications is less than $10^{-19} \mathrm{~cm}^{2}$ at a wavelength $\lambda=1532 \mathrm{~nm}$ [159]. Furthermore, visible and near-infrared transitions in solid state laser materials have cross sections in the range of $C_{\text {abs }}=10^{-20}$ to $10^{-18} \mathrm{~cm}^{2}$. Gas lasers in the same wavelength range show cross sections of $C_{\mathrm{abs}}=10^{-13}$ to $10^{-11}$ $\mathrm{cm}^{2}$ [75]. Therefore, cross-sections of quantum dots are relatively large.

The oscillator strengths $f_{\text {abs }}(E)$ of the low-energy absorption transitions in Figure 3.4 is calculated using the approach in Reference $[157]^{\text {vii }}$. Hence,

$$
f_{\mathrm{abs}}(E)=\frac{2 \ln (10) \epsilon_{0} c m_{\mathrm{e}} \sqrt{\epsilon_{\mathrm{m}}}}{e^{2} \pi \hbar N_{A}} \frac{1}{\left|F_{\mathrm{LF}}\right|^{2}} \int_{1 S_{h} \rightarrow 1 S_{e}} \epsilon_{\mathrm{ext}}(E) \mathrm{d} E,
$$

with $\epsilon_{0}$ being the dielectric constant of vacuum, $\epsilon_{\mathrm{m}}$ the dielectric constant of the medium (hexane), $c$ the speed of light in vacuum, $m_{\mathrm{e}}$ the electron mass, $d$ the di-

\footnotetext{
${ }^{\text {vii }}$ We corrected for an elementary charge $e$, which disappears in their derivation. Hence, the denominator contains $e^{2}$, in contrast to the $e$ term in the original paper [157].
} 
ameter of the quantum dot, $e$ the electron charge, $\hbar$ the reduced Planck constant, $F_{\mathrm{LF}}$ the local field factor, and $N_{A}$ denotes Avogadro's number. The integral runs over the narrow energy range of the first exciton transition (i.e., the hatched transitions from Figure 3.4). The oscillator strength is assumed to be constant in this energy range [157]. The local field factor is needed to compensate for the dielectric contrast between the dot and its surrounding medium. This contrast causes the electric field inside the dot $\mathbf{E}_{\text {in }}$ to be different from the field outside the $\operatorname{dot} \mathbf{E}_{\text {out }}$, such that $\mathbf{E}_{\text {in }}=F_{\mathrm{LF}} \mathbf{E}_{\text {out }}$. For spherical dots with a dielectric constant $\epsilon_{\mathrm{d}}$ inside a medium of $\epsilon_{\mathrm{m}},\left|F_{\mathrm{LF}}\right|$ is given by [160]

$$
\left|F_{\mathrm{LF}}\right| \approx \frac{3 \epsilon_{\mathrm{m}}}{\epsilon_{\mathrm{d}}+2 \epsilon_{\mathrm{m}}}
$$

Substitution of Equation 3.7 into Equation 3.6 and using the hatched regions from Figure 3.4 the absorption oscillator strengths of the quantum dots become ${ }^{\text {viii }}$ :

$$
\begin{aligned}
& f_{\text {abs }}=6.9 \pm 0.5 \text { (small dots), and } \\
& f_{\text {abs }}=10 \pm 1 \text { (large dots), }
\end{aligned}
$$

where the error stems from an estimation of the uncertainty in the measured $\epsilon_{\text {ext }}(E)$ (see offset in Figure 3.4). Note that $f_{\text {abs }}$ is not necessarily the same as the emission oscillator strength [162], as discussed below (after Equation 3.10). A systematic overestimation may arise from the integral over $E$ factor. If the absorption spectrum shows considerable inhomogeneous broadening, the integral is calculated over an energy range that is too large. The fact that the oscillator strengths exceed 1 does not violate the Thomas-Reiche-Kuhn sum rule because the number of electrons in a quantum dot is large. Furthermore, these values are in excellent agreement with earlier results [157]. Note that the oscillator strength increases with the nanocrystal size, whereas enhancement with small size was expected [163], and effective mass calculations predicted a size-independent oscillator strength [164-167]. Inside surroundings with a homogeneous refractive index $n=1.15$ (the effective refractive index of a titania inverse opal) the oscillator strength would be 1.8 times the values given in Equation 3.8.

\subsubsection{Emission spectra and Stokes shift}

The emission spectrum of the PbSe quantum dots in hexane suspensions are plotted in Figure 3.5. Figure 3.5. (a) shows the data for the smaller dots and in Figure 3.5.(b) are the data for the larger dots. Besides the emission spectra, the corresponding absorbance is reproduced from the experiment in Figure 3.4. For this emission experiment a HeNe laser $(\lambda=632.8 \mathrm{~nm} \Rightarrow E=1.96 \mathrm{eV})$ was used to excite the

\footnotetext{
viii Use $\epsilon_{0}=8.854 \cdot 10^{-12}[\mathrm{~F} / \mathrm{m}], c=2.998 \cdot 10^{8}[\mathrm{~m} / \mathrm{s}], m_{\mathrm{e}}=9.109 \cdot 10^{-31}[\mathrm{~kg}], e=1.602$. $10^{-19}[\mathrm{C}], \hbar=1.055 \cdot 10^{-34}[\mathrm{~J} \cdot \mathrm{s}], N_{A}=6.022 \cdot 10^{23}[1 / \mathrm{mol}], \epsilon_{\mathrm{m}}=1.864(\mathrm{see}[161])$, and $\epsilon_{\mathrm{d}}=23$ (see $[68,98,157])$. The integral term in Equation 3.6 yields $[\mathrm{eV} /(\mathrm{M} \cdot \mathrm{cm})]$, which must be converted to $\left[\mathrm{J} \cdot \mathrm{m}^{2} / \mathrm{mol}\right]$ by multiplying with $1.602 \cdot 10^{-20}$. Finally, recall that dimension $\mathrm{F} \cdot \mathrm{kg} \cdot \mathrm{m}^{2} /\left(\mathrm{C}^{2} \cdot \mathrm{s}^{2}\right)=1$.
} 


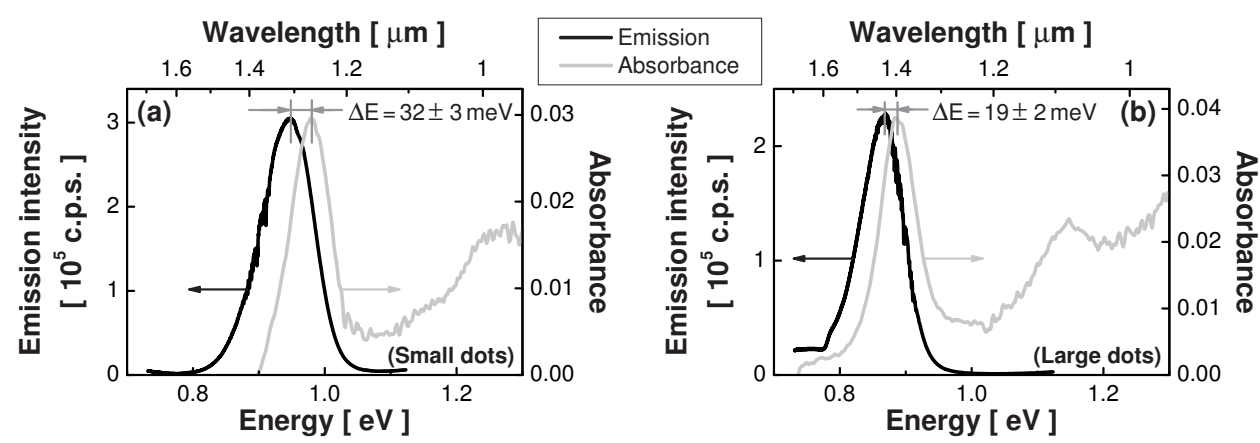

Figure 3.5: Emission and absorbance spectra of PbSe quantum dots suspended in hexane. (a) Data for dots with diameters $\phi \approx 4.7 \mathrm{~nm}$ (PbSe1310). (b) Data for dots with diameter $\phi \approx 5.1 \mathrm{~nm}$ (PbSe1550). Arrows connected to the curves point towards the corresponding ordinate. The derived difference in emission and absorbance peak position $(\Delta E)$ corresponds to the non-resonant Stokes shift. The absorbance data stems from Figure 3.4.

dots non-resonantly with a power of $4.87 \mu \mathrm{W}$. The emission peak shifts to larger energies if the dots are smaller. This is in correspondence with quantum size effects.

As expected the emission spectrum is slightly red-shifted with respect to the absorption spectrum [151]. The energy difference between the absorbance and the emission peak corresponds to the non-resonant Stokes shift. As depicted in Figure 3.5 Stokes shifts of $32 \pm 3 \mathrm{meV}$ and $19 \pm 2 \mathrm{meV}$ are derived for the small and large dots respectively. In Figure 3.6 these results are combined with results that we have derived from spectra in the literature $[68,129,136]$. The different measurements all show an increase of the Stokes shift with decreasing quantum dot size. The data from the different papers are offset with respect to one another, which may be due to a difference in the type of capping layer used to passivate the dots. Our measured Stokes shifts are relatively small, and agree with the data from $[68,129,136]$. The small Stokes shift is consistent with band-edge emission, and is only about $4 \%$ of the confinement energy. For comparison, monodisperse CdSe nanocrystal solutions exhibit larger non-resonant Stokes shift of about $10 \%$ to $15 \%$ of the confinement energy [98, 131, 168]. The small Stokes shift of PbSe nanocrystals is not only a reflection of the narrow size dispersion of the sample, but may also be indicative of the simpler exciton structure as compared with CdSe $\operatorname{dots}[68]$.

\subsubsection{Homogeneous vs inhomogeneous linewidth}

The emission linewidth of the smaller quantum dots from Figure 3.5.(a) is 93 meV (full width at half maximum). This corresponds to a relative linewidth of $0.093 / 0.95=9.8 \%$. The width of the emission spectrum is broadened as a re- 


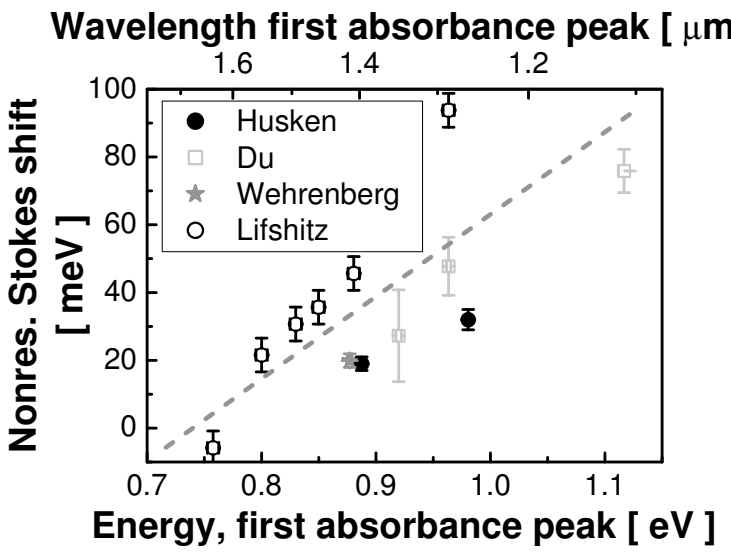

Figure 3.6: Nonresonant Stokes shift of several PbSe quantum dot suspensions versus the first absorbance peak energy. Smaller quantum dots (absorbance peak at high energies) reveal larger Stokes shifts. Black dots are our data. Open squares are data that we derived from [136], gray star is derived from [68], open circles are derived from [129]. The large error bars in the second data set are caused by structure in the emission spectrum, that makes it difficult to determine the emission peak energy. The last data set even shows a negative Stokes shift for the largest quantum dots. The dashed line is a guide to the eye.

sult of intrinsic (homogeneous) and extrinsic (inhomogeneous) effects. The former includes dephasing due to the exciton-phonon interaction and carrier-carrier (or exciton-exciton) interactions. The inhomogeneous broadening can be attributed to the size and shape dispersions, and scattering of the surface defects [99]. The question remains how much the emission linewidth is determined by (in)homogeneous broadening.

Additional emission experiments were done in a different experimental setup, which allowed for resonant excitation of the quantum dots. The full width at half maximum time duration of the tunable laser pulses is 120 to $140 \mathrm{fs}$ [169]. Figure 3.7.(a) shows the absorbance measured using pulsed excitation (squares) as compared to the earlier results using white light illumination (Figure 3.4.(a)). The absorbance peak energy agrees with the linear measurements, and the maximum absorbance of 0.03 shows the low optical density of the sample. The center of the absorbance peak is reduced with respect to the earlier measurement, perhaps as a result of relatively large standard deviations in the measured signals. The relative uncertainty in the measurement is about $6 \%$, both for the transmitted intensities trough the quantum dot suspension and for the reference sample. Figure 3.7.(b) shows the emission intensity as a function of the excitation or pump intensity. We have converted this quantity to the mean number of excitons $\left\langle n_{\mathrm{x}}\right\rangle$ created in a single dot (Equation 3.2). This conversion reveals that we scan from a low to a 

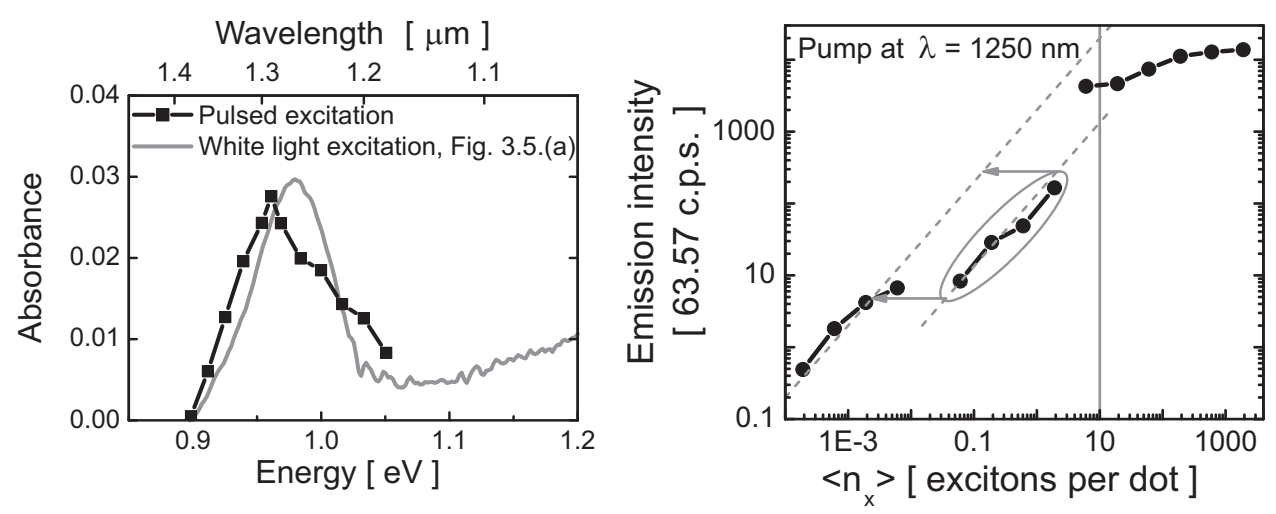

Figure 3.7: (a) Black squares result from absorbance scan using pulsed laser excitation. Gray line shows result from Figure 3.4.(a). A maximum absorbance of approximately 0.03 was measured which confirms the sample being optically thin. (b) Peak emission intensity versus the average number of excitons $\left\langle n_{\mathrm{x}}\right\rangle$ created per quantum dot. Excitation at $\lambda=1250 \mathrm{~nm}$. The emission intensity is linear with $\left\langle n_{\mathrm{x}}\right\rangle$ for $\left\langle n_{\mathrm{x}}\right\rangle<10$ and saturates for larger $\left\langle n_{\mathrm{x}}\right\rangle$. Four points are offset systematically. These points are measured with a neutral density filter not used in the other measurements. These points do show the linear dependence of emission intensity on fluence. We believe the optical density of the filter was underestimated by approximately 10 times. Dashed lines correspond to a linear dependence of emission intensity on excitation fluence. The vertical line is a guide to the eye. In both figures (a) and (b) the symbols denote the measured data, and the connecting lines are guides to the eye.

very high excitation regime. The emission intensity is linearly dependent on $\left\langle n_{\mathrm{x}}\right\rangle$ for $\left\langle n_{\mathrm{x}}\right\rangle<10$. For $\left\langle n_{\mathrm{x}}\right\rangle>10$ the emission intensity saturates. The onset of the saturation at this surprisingly large value of $\left\langle n_{\mathrm{x}}\right\rangle=10$ seems to be consistent with experiments where many excitons are excited in PbSe quantum dots by high energy pump photons $[138,140]$.

Figure 3.8 shows the result of a fluorescence linewidth narrowing experiment where the excitation wavelength is scanned over the quantum dots' emission spectrum. Figure 3.8.(a) shows three emission measurements: one excited at the red side of the emission spectrum, one excited to the blue side of the emission spectrum, and one excited at the far blue (non-resonant excitation). Although the emission intensities vary a lot, the spectral shape is very much alike. Figure 3.8.(b) shows the relative linewidth of the emission spectrum as a function of the excitation photon energy (open circle, and closed circles). Clearly the linewidth is practically constant at $10 \%$, which corresponds to an average width of $97 \mathrm{meV}$. (Aside, the relative linewidth of the laser is $1.5 \%$.) As a measure of the excitation efficiency the measured emission intensity is divided by the excitation power, and normal- 


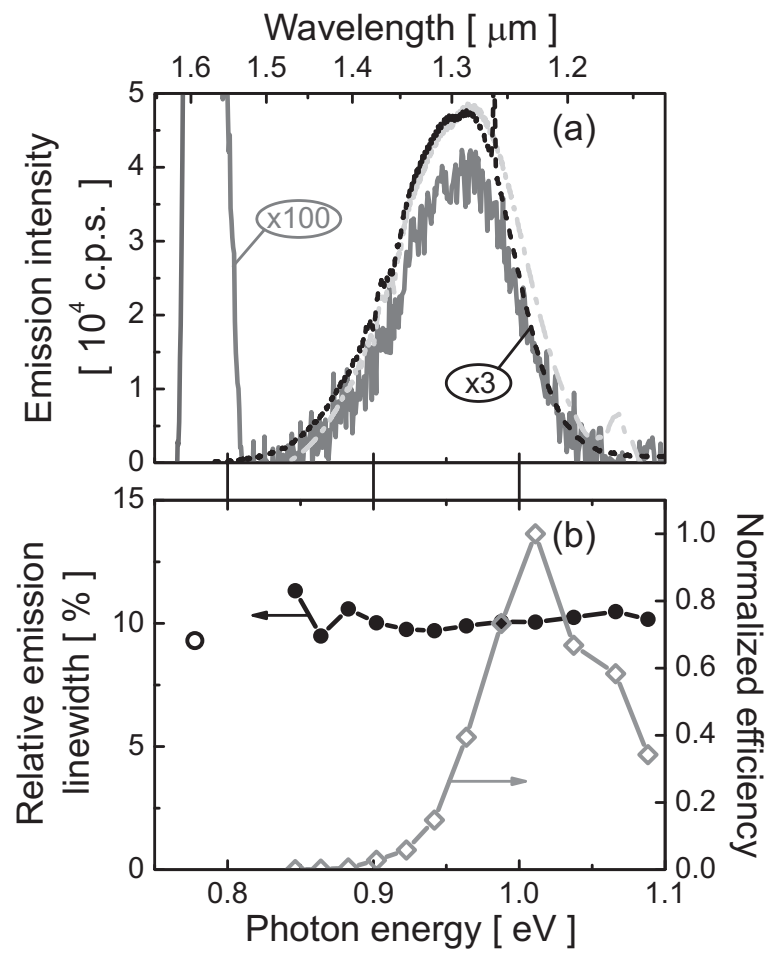

Figure 3.8: (a) Emission from PbSe quantum dots in hexane measured with three different excitation photon energies: 1) dark gray line uses excitation at $0.78 \mathrm{eV}$, 2) light gray dash-dotted line uses excitation at $1.07 \mathrm{eV}, 3)$ black dashed line uses non-resonant excitation at $1.96 \mathrm{eV}$. All emission spectra have approximately the same shape and width. (b) Relative linewidth versus excitation energy (open and closed circles). The linewidth is approximately constant. Open diamonds denote the normalized ratio between the emission and the excitation power. The sample is most efficiently excited at $1.01 \mathrm{eV}$. Horizontal arrows point towards the relevant axis.

ized. This yields an excitation optimum at photon energy $1.01 \mathrm{eV}(1230 \mathrm{~nm})$. The excitation power at the lowest pump energy $(\lambda=1600 \mathrm{~nm})$ is $29 \mu \mathrm{W}$, which corresponds to an average number of excitons $\left\langle n_{\mathrm{x}}\right\rangle=9.3 \cdot 10^{15} \cdot C_{\mathrm{abs}}(1600 \mathrm{~nm}) \approx 0$, as the absorption cross section of these smaller dots vanishes at these large wavelengths (see Figure 3.4). The excitation intensity ${ }^{\text {ix }}$ corresponds to approximately $8 \mathrm{GWcm}^{-2}$. This is only about one to two orders of magnitude smaller than the pump intensities used in optical switching experiments with non-linear, two photon absorption $[169,170]$. The broad and constant width of the emission spectrum is

\footnotetext{
${ }^{\mathrm{ix}}$ using $\Phi(0)$ from Equation B.10 times the pulse power, i.e., the energy per pulse divided by the pulse duration.
} 
therefore suggested to be caused by a two-photon absorption process, that causes the quantum dots to be effectively excited non-resonantly.

Fluorescence line-narrowing spectra were taken by Lifshitz et al. from similar ensembles of PbSe quantum dots with about the same relative linewidth [129]. These experiments indicate a negligible reduction of the width of the resonant photoluminescence band with respect to the width of the nonresonant photoluminescence band, suggesting that the contribution of inhomogeneous broadening due to size distribution is minor. This small inhomogeneous broadening seems surprising as the strong confinement in PbSe dots should reduce the exciton-phonon coupling and lead to smaller intrinsic line widths as compared to CdSe quantum dots, that have sub meV homogeneous widths [67]. However, the broad homogeneous linewidth may be explained by the peculiar electronic structure of PbSe. There are four equivalent extrema (valleys) in the Brillouin zone, $\mathrm{S}$ and $\mathrm{P}$ levels arising from these valleys (and from the two directions of spin) are split by anisotropy, spin-orbit, and intervalley couplings [98, 141]. A $50 \mathrm{meV}$ homogeneous width can be associated with the energy distribution of the band-edge states $^{\mathrm{x}}[98,146,172]$. The large homogeneous linewidth corresponds to fast dephasing times $<100$ fs [172]. This fast dephasing may make these $\mathrm{PbSe}$ quantum dots unsuitable for applications that need coherence control. The emission spectrum observed in Figure 3.8 (and also later in Figure 4.7) is broader than $50 \mathrm{meV}$ and must therefore be inhomogeneously broadened. From this brief study our quantum dot ensemble in hexane seems to be inhomogeneously broadened by only about two times the homogeneous linewidth. This conclusion is substantiated in the following section.

\subsection{Time-resolved emission and oscillator strength}

Besides the emission spectra the time-resolved radiative decay of the quantum dot ensemble was determined. Figure 3.9 shows the decay curves of PbSe quantum dots inside a hexane suspension, and inside a non-photonic titania inverse opal. For each sample, three different wavelengths are considered. The decay is single exponential in the hexane suspension, with decay rates $\Gamma_{1250}=0.71 \mu \mathrm{s}^{-1}, \Gamma_{1300}=0.73 \mu \mathrm{s}^{-1}$, and $\Gamma_{1450}=0.79 \mu s^{-1}$ (subscripts denote wavelength in nanometers). This difference in decay rate is a direct evidence that the emission spectrum is inhomogeneously broadened. If the width of the emission spectrum would be only homogeneously broadened, the decay rate should have been the same, no matter at which energy the system is probed.

The decay curves measured from the inverse opals are non-exponential (Figure 3.9.(b)). These curves will be modeled in Chapters 5 and 6 to find corresponding average decay rates. For the moment it is only important to note that the longer wavelength shows faster decay, similar to the suspension above. Hence, the decay rate $\Gamma$ increases with increasing quantum dot size or decreasing frequency. This

\footnotetext{
${ }^{\mathrm{x}}$ For PbS quantum dots, single dot linewidths of $100 \mathrm{meV}$ were recently measured, at somewhat shorter wavelengths [171].
} 

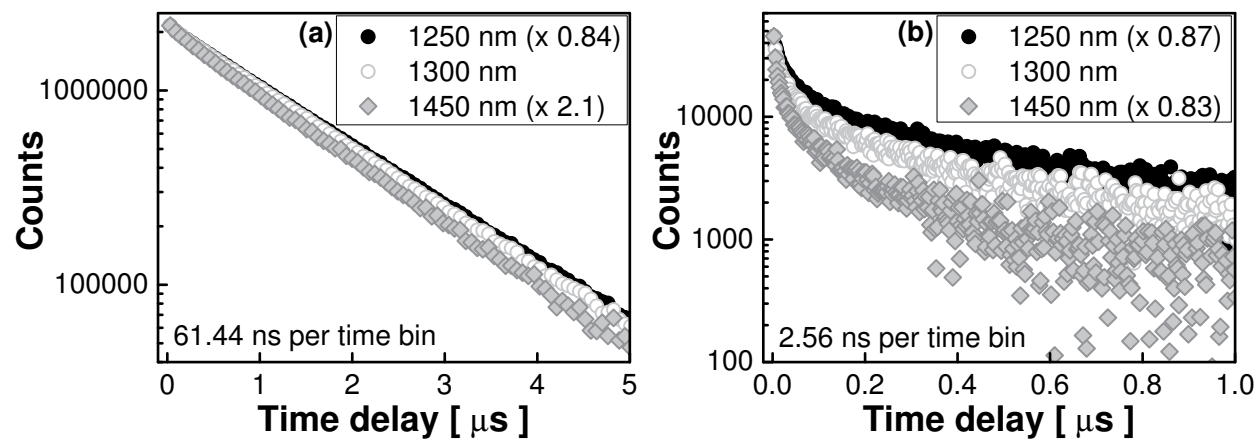

Figure 3.9: Spontaneous emission intensity decay of PbSe quantum dots: (a) dots suspended in hexane, (b) dots inside a non-photonic titania inverse opal (lattice parameter $a=369 \mathrm{~nm})$. Measurements were done at three different wavelengths: $\lambda=1250 \mathrm{~nm}$ (black dots), $\lambda=1300 \mathrm{~nm}$ (open circles), and $\lambda=1450 \mathrm{~nm}$ (gray diamonds). The decay curve is single exponential in the hexane suspension and nonexponential in the crystal. In both samples the decay rate increases with wavelength. To reduce the Poisson noise in the data time bin sizes were increased by summing counts of adjacent bins. The decay curves are scaled to the maximum counts of the $\lambda=1300 \mathrm{~nm}$ measurement for clarity.

behavior is opposite to the expectation for an ideal exciton that the decay rate is proportional to the emission frequency $\omega$, i.e., $\Gamma_{j}=$ constant $\cdot \omega_{j}[167,173]$. Previous calculations and experiments on PbSe quantum dots indeed showed a lifetime increase with quantum dot size $[132,136]$. However, these calculations do not take into account the non-stoichiometry of the dots [157], and the experimental data presented is incomplete.

There are two hypotheses for the increase of the quantum dot decay rate with size. (i) The non-radiative decay rate may become larger for larger dot sizes, due to phonon coupling. This essentially means that the changes in decay rate are due to changes in the quantum efficiency of the dots. However, phonon interactions are expected to be small due to the strong confinement that are especially important in PbSe quantum dots $[67,132]$. This idea is supported by the weak temperature dependence of the spectrum and decay rate of PbSe quantum dots [132, 133], which was not observed in other semiconductors. (ii) The other explanation for the changes in decay rate are related to the difference in oscillator strength of the dots. Therefore, the decay rate is written explicitly as a function of the emission oscillator strength $f_{\mathrm{em}},[75]$

$$
\Gamma_{\mathrm{rad}}^{\mathrm{hom}}\left(\omega_{i}\right)=\frac{e^{2} n \omega_{i}^{2}}{6 m_{\mathrm{e}} \epsilon_{0} \pi c^{3}} f_{\mathrm{em}}\left(\omega_{i}\right) .
$$

Here, $\Gamma_{\mathrm{rad}}^{\mathrm{hom}}(\omega)$ is the radiative decay rate inside a homogeneous medium. The other symbols are as described before (see Equation 3.6). Subsequently the ratio of the 
emission oscillator strengths can be determined using Equation 3.9, the results from Figure 3.9.(a) and considering $\omega_{i}=2 \pi c / \lambda_{i}$. This yields

$$
\frac{f_{\mathrm{em}}(1450 \mathrm{~nm})}{f_{\mathrm{em}}(1250 \mathrm{~nm})}=\frac{\Gamma_{\mathrm{rad}}^{\mathrm{hom}}(1450 \mathrm{~nm}) \cdot 1450^{2}}{\Gamma_{\mathrm{rad}}^{\mathrm{hom}}(1250 \mathrm{~nm}) \cdot 1250^{2}}=\frac{0.79}{0.71} \cdot \frac{1450^{2}}{1250^{2}}=1.5 .
$$

Hence, the oscillator strength $f_{\mathrm{em}}$ of the larger dot, emitting at longer wavelength, is about 1.5 times larger than the oscillator strength of the smaller dots. This result is in very good agreement with the ratio of the oscillator strengths determined from the absorption measurements in Section 3.4.1. There, the oscillator strength of the larger dots was 1.4 times larger than that of the smaller dots (see Equation 3.8), where the wavelength difference of the two absorption peaks was somewhat smaller than the difference used in this section. Furthermore, this increase in oscillator strength with quantum dot size was observed experimentally and predicted by tightbinding calculations [157]. An increase of oscillator strength with quantum dot size was also observed for InAs quantum dots [174]. We therefore believe that the oscillator strength causes the change in the decay curves, instead of phonon-coupling processes.

From Equation 3.9 it is also possible to calculate the value for the emission oscillator strength, which yields $f_{\mathrm{em}}(1300 \mathrm{~nm})=0.041$. However, the above expression does not take into account the screening of the radiating field inside the quantum dot. If the nanocrystal is spherical, the internal field is reduced by $F_{\mathrm{LF}}$ (see Equation 3.7). Compensating for this local field factor $F_{\mathrm{LF}}$, like in Equation 3.6, yields an emission oscillator strength of $f_{\mathrm{em}}(1300 \mathrm{~nm})=0.92$, which is 7.5 times smaller than the absorption oscillator strength. The absorption oscillator strength is not necessarily equal to the emission oscillator strength. In emission, one is only sensitive to quantum dots that are emitting light, while absorption measurements probe all quantum dots in the ensemble [162]. For comparison, theory predicted the oscillator strength of PbSe quantum dots to be somewhat smaller than that of CdSe quantum dots. This is caused by the inter-valley coupling among the four equivalent $\mathrm{L}$ points in the Brillouin zone of the PbSe dots. This coupling mixes the phases of the wave functions, which reduces the oscillator strength [132]. The large oscillator strength of PbSe quantum dots make the dots very attractive to quantum electrodynamics experiments [174].

\subsection{Conclusions and remarks}

We have described and characterized the PbSe quantum dots used in the remainder of this thesis. The photoluminescent quantum efficiency was estimated to be larger than $30 \%$. Absorption cross sections on the order of $10^{-16}$ to $10^{-15} \mathrm{~cm}^{2}$ were determined, in good agreement with the literature. Both the absorption and emission oscillator strengths were derived from experiment. Absorption oscillator strengths from 6.9 to 10 were found, which agree with recent publications and which are ap- 
proximately 7.5 times larger than the emission oscillator strength. Furthermore we found the oscillator strength to increase with quantum dot size, giving rise to an increase of the radiative decay rate with size.

The width of the quantum dot emission spectrum is estimated to be inhomogeneously broadened by at most a factor of two. The fast dephasing times $<100 \mathrm{fs}$ corresponding to the large homogeneous linewidth may disqualify these quantum dots for applications that need coherence control.

The large dielectric constant, together with the large exciton Bohr radius and the four equivalent $\mathrm{L}$ points in the Brillouin zone, make PbSe quantum dots special quantum dots. The dots' large dielectric constant gives rise to strong screening effects that influence the response of the quantum dots to external fields and give rise to decay rates that are relatively slow compared to other semiconductor quantum dots. Still, the decay rates are orders of magnitude faster than that of erbium, the standard light source for telecom applications at these near-infrared wavelengths.

Using the results in this chapter we are able to interpret quantum dot emission spectra and decay rates. Therefore, we can start to study the dots in complex environments, like photonic crystals. In Chapter 4 emission spectra of quantum dots inside photonic crystals are studied. In Chapters 5 and 6 spontaneous emission decay dynamics is studied in detail. 


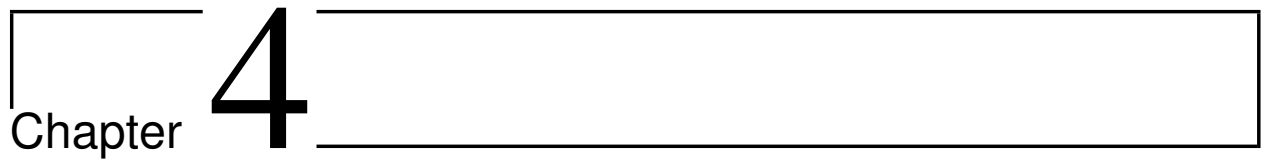

\section{Angular redistribution of near-infrared emission from quantum dots in 3D photonic crystals}

\subsection{Introduction}

There is currently much interest in both light propagation and light emission phenomena. Light propagation is studied in isotropic, random media, already for a long time [175-180]. Emission properties of light sources like atoms, molecules, and quantum dots are also studied intensively. It was recognized that the spontaneous emission by atoms can be controlled by modification of the properties of the radiation field [6]. Later, it was realized that periodic, dielectric structures can be used to inhibit spontaneous emission completely [1]. This paper opened a new field in solid-state physics where spontaneous emission is a limiting factor in numerous devices. It suddenly became important to know the optical properties of these dielectric structures, or photonic crystals. Therefore, light propagation experiments were done and theory was developed to explain the characteristic properties of the photonic crystals [56, 181-187]. Furthermore, emission spectra were measured from light sources embedded inside photonic crystals [81-84, 188-197]. These studies showed that optical Bragg diffraction inside the photonic crystals strongly influences the propagation of light.

One of the central issues regarding light transport is the angular distribution, or escape function, of the emitted light that exits a scattering medium. Fundamental insight in this escape probability distribution is needed to explain the light transport 
properties of the photonic crystal $[198,199]$. Surprisingly, the escape function is often disregarded. In emission measurements this disregard easily leads to misinterpretations of the experimental results. Changes in emission power, measured from light sources inside photonic crystals, are mistakenly attributed to enhancement or inhibition of the sources' spontaneous emission decay rate [188, 192, 200-202]. However, to make such claims the emission spectrum of the light source inside the photonic crystal should be compared to the emission spectrum of the source inside a non-photonic reference sample. For this comparison detailed knowledge about the escape function is needed ${ }^{\mathrm{i}}[15,82]$.

Most experimental work on light emission in photonic crystals was limited to the visible range. For future experiments on very strongly interacting photonic crystals made of silicon, near-infrared wavelengths $(\lambda>1100 \mathrm{~nm})$ are important. This wavelength range also includes the range for telecom applications. Here, emitted light escaping from 3D photonic crystals is studied at near-infrared wavelengths. Colloidal PbSe quantum dots are used as light sources inside titania inverse-opal photonic crystals. This chapter summarizes 25 angle-resolved emission experiments using 11 different lattice parameters. Section 4.2 considers the sample fabrications, preparation, and handling as well as the measurement procedure. Subsequently, the results are presented and discussed in Section 4.3. Conclusions form the last part of this chapter, Section 4.4. A detailed description of the experimental setup is given in Chapter 2.

\subsection{Experimental details}

\subsubsection{Fabrication and characterization of the photonic crys- tals}

The photonic crystals consist of air spheres in anatase titania. See References [58, 203] for a detailed description of the fabrication and characterization of these crystals. The crystals are made by filling the voids in artificial polystyrene opals. Subsequently, the polystyrene spheres are removed by calcination. The pores are arranged in a face centered cubic (fcc) lattice. X-ray diffraction and scanning electron microscope studies show that the direction perpendicular to the samples' surface corresponds to the (111) direction of the crystal [58, 204].

The quantum dots that will be embedded inside these crystals emit at near infrared frequencies (wavelength $\lambda \approx 1300 \mathrm{~nm}$ ). Braggs' law is used to derive the required size of the polystyrene spheres that yields the crystals' lowest order stop band at the same frequency. Therefore, Equation 1.3 is rewritten in the form

$$
\lambda_{111}=\frac{4}{3} \sqrt{6} n_{\mathrm{eff}} r \cos \left(\Theta_{\mathrm{E}}\right)=1300[\mathrm{~nm}] .
$$

\footnotetext{
'If the light source is very efficient claims on the sources' decay rate can only be inferred from time-resolved measurements (see Chapter 6, and [15]).
} 
Here, $r=r_{\text {sphere }} \cdot \mathrm{F}_{\text {shrink }}$ is the radius $r_{\text {sphere }}$ of the polystyrene spheres times a factor $F_{\text {shrink }}$ that accounts for the shrinking of the crystal during the calcination process [58]. Radii larger than the one that fulfils Equation 4.1 can be used, as the resulting crystals will Bragg diffract the $\lambda=1300 \mathrm{~nm}$ light at larger external angles. Much smaller radii yield crystals that do not Bragg diffract these wavelengths and are used as a reference. A typical $30 \%$ shrinking of the crystal during fabrication [58] is used (hence, $\mathrm{F}_{\text {shrink }}=0.70$ ). As a result, polystyrene sphere radii in the range of $r=340$ to $620 \mathrm{~nm}$ are needed to obtain the L-gap around $\lambda=1300 \mathrm{~nm}$. Additionally, $r<200 \mathrm{~nm}$ spheres are used for the non-photonic reference samples.

Not all fabricated structures are suited for emission experiments. Parts of the crystals may be covered with bulk titania, or the air-sphere ordering may be poor. By eye, samples that show clear Bragg diffraction are selected for further study. Subsequently, bright-field microscope images are made to chart each sample. Reflectivity measurements are done to find the regions suitable for emission experiments. Figure 4.1 shows a typical titania inverse opal with the result of four reflectivity measurements. The color of the sample is caused by higher order Bragg diffraction. The first spectrum shows clear Bragg diffraction from the (111) planes. Measurements two and three show diffraction peaks that are too weak for these areas to be of further use. The fourth measurement is taken at a position where the crystal is covered with bulk titania. As clearly confirmed by the reflectivity measurements, the sample has both useful photonic crystal regions and inferior parts. In case of Figure 4.1, only the first location is suitable for emission experiments. Clearly, reflectivity measurements are needed to reveal the areas that we can use.

Reflectivity measurements on four samples with different lattice parameters are shown in Figure 4.2. The reflectivity setup was described in References [205, 206]. Only results that show clear Bragg diffraction are shown. The emission spectrum of $\mathrm{PbSe}$ quantum dots is included in the same graph. The stop bands are overlapping or to the red of the quantum dot emission spectrum, and far to the blue of the emission spectrum for reference measurements. Therefore, the fabricated samples cover the desired wavelength range.

An overview of the reflectivity measurements is given in Figure 4.3. In Figure 4.3. (a) the central wavelength $\lambda_{111}$ of the stop band is plotted versus the radius of the spheres $r_{\text {sphere. }}$ The linear dependence of $\lambda_{111}$ on $r_{\text {sphere }}$, expected from Equation 4.1, is very clear. Furthermore, the new data points (plus signs) are in excellent agreement with earlier results from [58] (circles). The slope of the line corresponds to an effective refractive index $n_{\text {eff }}=1.17$, also observed in References [58, 208]. Using a refractive index of 2.4 [209] for anatase titania yields a filling fraction of $12 \%$ solid material. $n_{\text {eff }}$ may slightly change due to changes in the shrinkage factor $\mathrm{F}_{\text {shrink }}=0.7$. Due to changes in local filling fractions and template shrinkage, there is a small variation in the measured values for $\lambda_{111}$ at a given radius. Higher order diffraction peaks (triangles, squares and stars) were observed in the expected frequency range. These peaks arise from complex coupling of many Bloch waves [207]. 

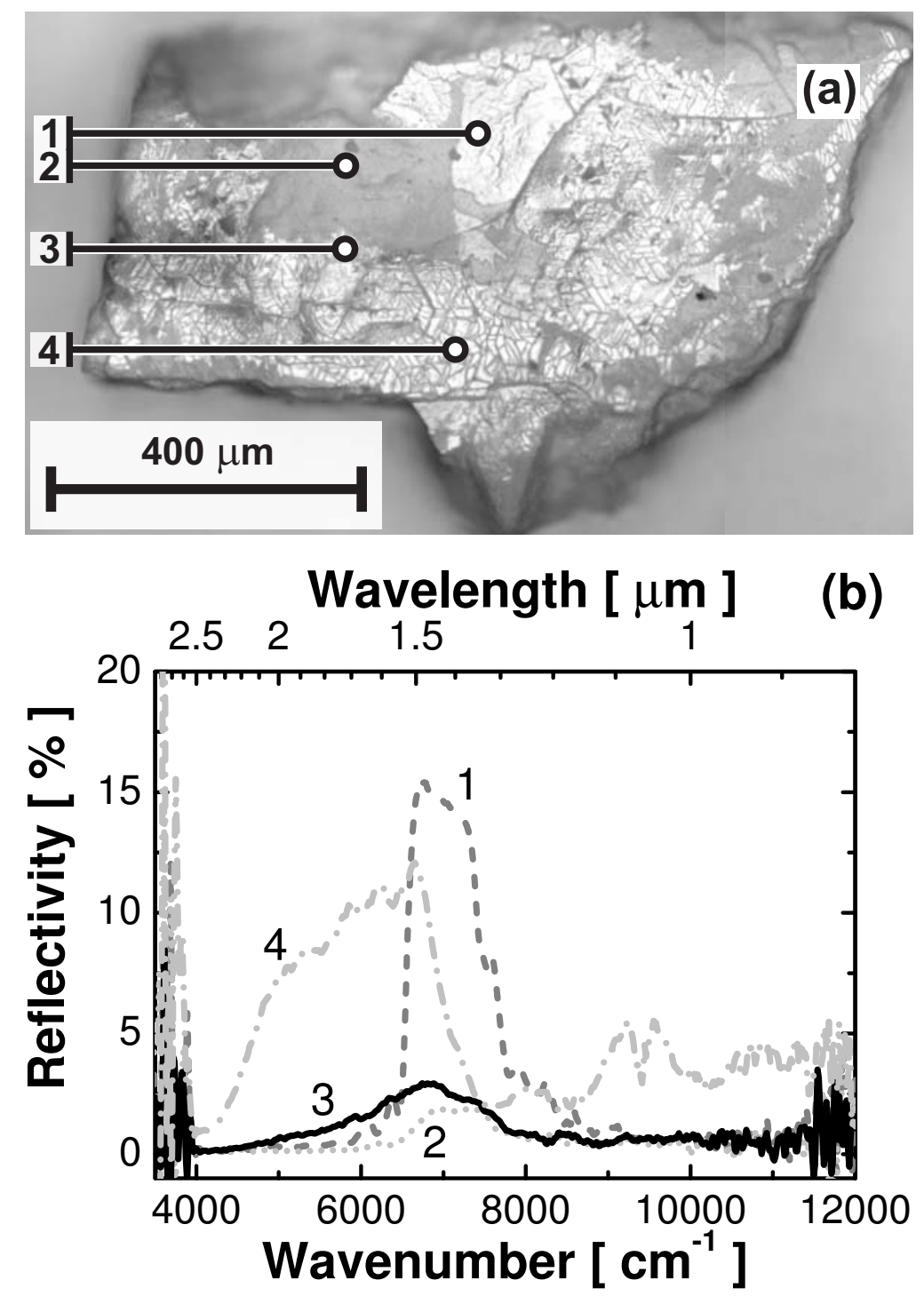

Figure 4.1: (a) Bright-field microscope image of a titania inverse opal. Numbered lines point to four locations from which reflectivity spectra were determined. (b) Four reflectivity spectra measured from the photonic crystal in (a). The labels correspond to the locations in (a). Only position number 1 corresponds to clear Bragg diffraction and is suitable for subsequent experiments. Position 2 and 3 show only very weak peaks, probably due to small crystal domains. Position 4 corresponds to a location that is covered with bulk titania. 


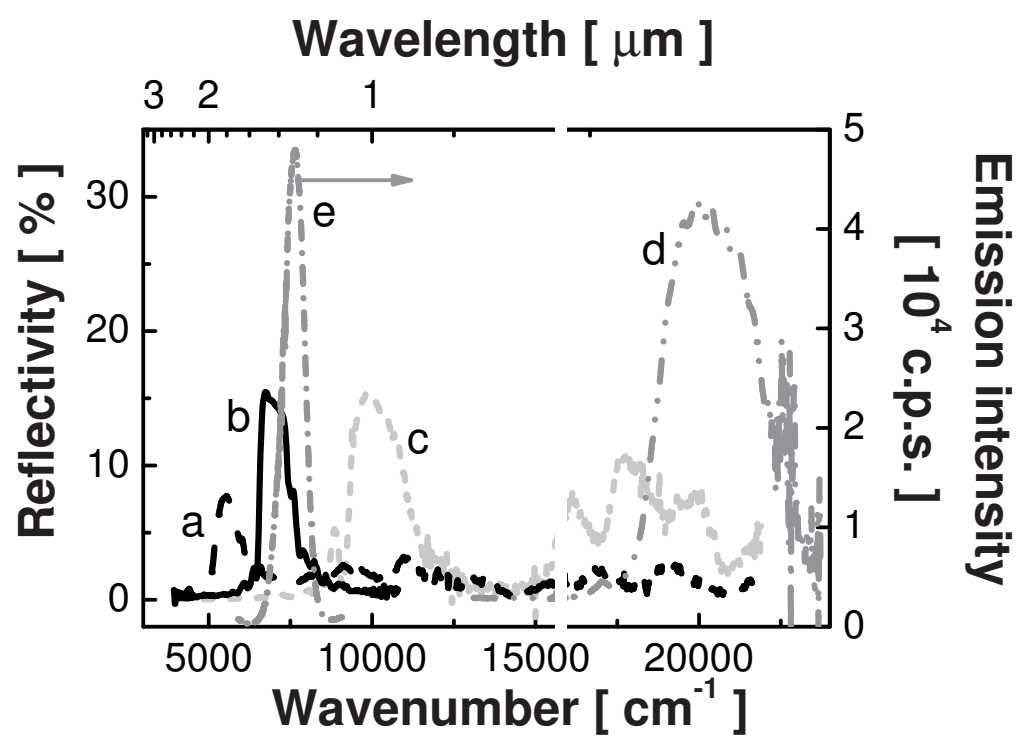

Figure 4.2: Normal-incidence reflectivity spectra measured from four titania inverse opals. These samples were grown from templates with different sphere radii $r_{\text {sphere }}$, namely: (a) $655 \mathrm{~nm}$, (b) $515 \mathrm{~nm}$, (c) $403 \mathrm{~nm}$, and (d) $180 \mathrm{~nm}$ (left ordinate). Stop bands due to the L-gap appear as peaks in the reflectivity spectrum. Curve (c) contains clear higher order diffraction peaks around $17,500 \mathrm{~cm}^{-1}$. (e) Shows the emission spectrum of $\mathrm{PbSe}$ quantum dots in a hexane suspension (right ordinate). The reflectivity is not defined in a narrow band around $15,800 \mathrm{~cm}^{-1}$ because of interference by a reference laser beam inside the spectrometer.

Figure 4.3.(b) shows the relative width of the stop band, which is an upper bound for the photonic strength. The average of all points is 0.142 , with a standard deviation of 0.025 . This average is in good agreement with earlier measurements in the visible range $[58,205,208]$. The photonic strength determined from bandstructure calculations on titania inverse opals is $0.11[14,210]$. Relative widths larger than this 0.11 are observed and due to finite size effects in the crystal. A reduced filling fraction of titania causes a decrease of the relative width [208].

A photonic strength of 0.14 is relatively large and shows the strong interaction of light with the crystal. The quality of the selected samples is comparable to samples used in earlier experiments in the visible range [30, 82, 194]. The areas that show the most powerful Bragg diffraction are used in subsequent emission experiments. 


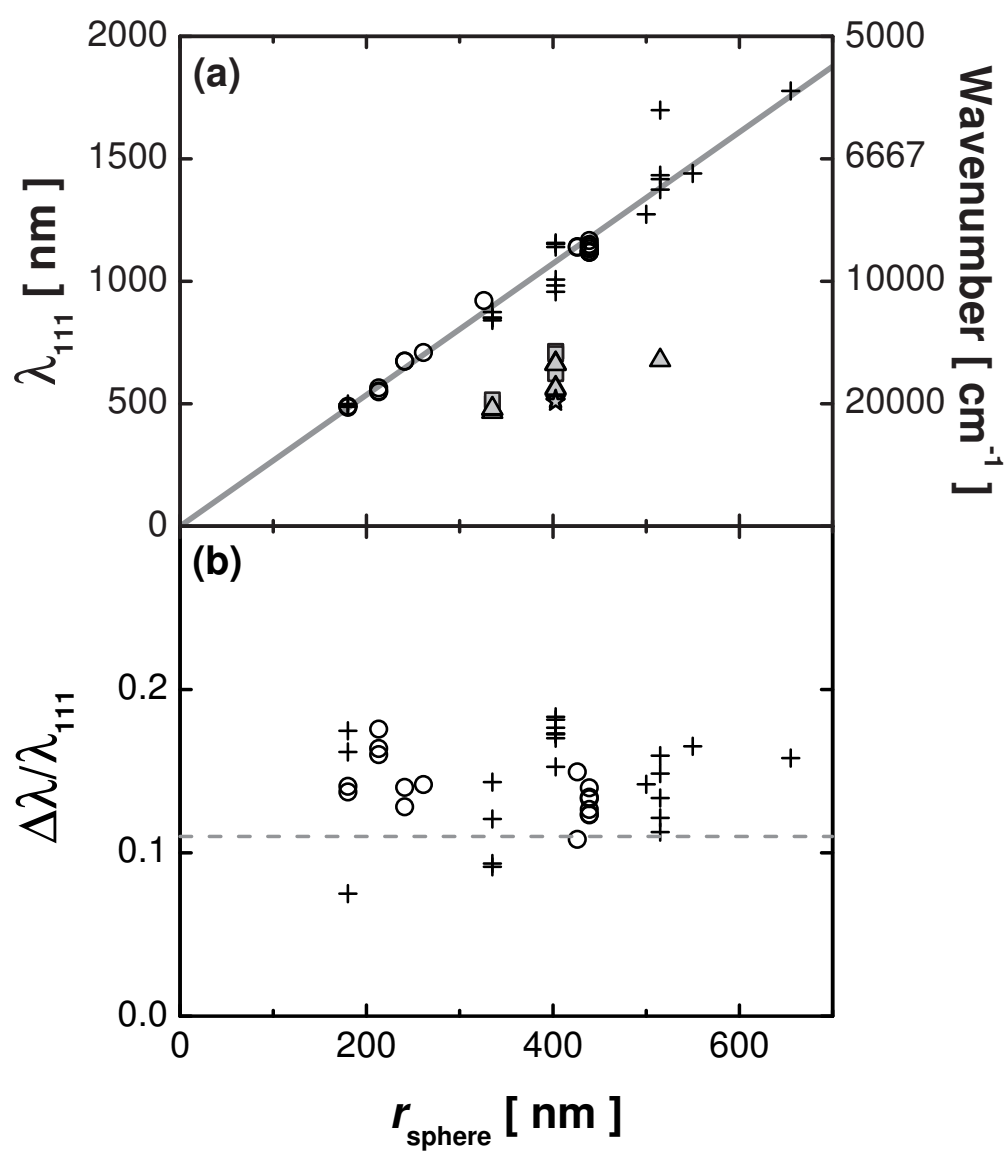

Figure 4.3: Measured stop band frequencies and their relative width versus the radius of the polystyrene spheres used to fabricate the titania inverse opals. Data open circles are included from [58], other symbols show our new results. (a) Stop band frequencies: plus signs and open circles correspond to the L-gap. Triangles, squares and stars are respectively the first, second, and third diffraction peak in the higher order diffraction triplet. This triplet is caused by multiple Bragg-wave coupling [207]. The gray line is a linear fit through the L-gap data points and the origin, i.e., $\lambda_{111}=2.68 \cdot r_{\text {sphere }}$ (b) Relative width of the L-gaps measured in (a). A large variation in relative width is observed. This measured width is an upper boundary for the samples' photonic strength [208]. From bandstructure calculations a photonic strength of 0.11 is expected [14] (horizontal dashed line). Values larger than this 0.11 are caused by finite size effects in the crystal, whereas smaller values result from a reduced filling fraction of titania. The results demonstrate the strong interaction of light with these structures. The horizontal dotted lines are guides to the eye. 


\subsubsection{Quantum dot infiltration and preparation for measure- ments}

After the fabrication and characterization of the photonic crystals, the suitable samples are infiltrated with colloidal PbSe quantum dots; similar to [30, 82, 191, 194]. Each sample is placed in a $5 \mathrm{ml}$ glass vial. Next, $50 \mu \mathrm{l}$ of $0.92 \mu \mathrm{M} \mathrm{PbSe}$ quantum dots in hexane suspension is added. After one day the suspension is removed and the samples are rinsed three times with hexane, each time for $20 \mathrm{~s}$. Subsequently, the sample is left to dry for one day. The infiltration leads to a distribution of quantum dots on the titania surfaces inside the inverse opals. From the concentration of dots in the suspension and the volume of the air spheres with typical radii of $385 \mathrm{~nm}$, the distance between adjacent quantum dots is estimated to be about $120 \mathrm{~nm}$. This is sufficiently large to avoid reabsorption and energytransfer processes between the dots [151]. In the results section of this chapter it is deduced that the quantum dots are really inside the photonic crystal.

Originally, butanol was added to the quantum dot in hexane suspension. This approach was successfully applied to CdSe quantum dots in Reference [30] to make the suspension more hydrophilic. The addition of a hydrophilic solvent should improve the infiltration of the structure with the suspension. However, the addition of butanol caused the PbSe quantum dots to cluster, as discussed in Appendix D.1. Therefore, this method is not suitable for the PbSe experiments. Clusters are not observed in the applied infiltration method using only quantum dots in hexane.

To prevent photo-oxidation of the quantum dots, the sample preparation and handling was carried out in a high quality nitrogen-purged glove box (MBraun, LabStar). For optical measurements the samples were glued to the side of a needle pin, mounted in a sealed chamber, and kept under a 0.6 bar nitrogen atmosphere. Figure 4.4.(a) shows the home-build chamber that can be mounted on a rotation stage via a threaded ring at the bottom. Between this ring and the chamber, stages are visible that can be used to tilt and translate the chamber. Figure 4.4.(b) contains an image of a sample glued to a needle pin. As only the small side of the crystal is in contact with the needle, both the frontside and backside of the sample remain accessible for the optical experiments, discussed below.

\subsubsection{Emission setup and measurement procedure}

Figure 4.5 describes the basic concept of the experiment. The sample is illuminated by a slightly focused laser beam of wavelength $\lambda=532 \mathrm{~nm}$. The excitation spot radius is estimated to be about $7 \mu \mathrm{m}$. Light emitted from the sample is collected by a 0.05 NA objective and sent to a spectrometer. An $850 \mathrm{~nm}$ long-pass filter blocks the laser light. The sample and excitation objective are rotated together, such that only the detection angle $\Theta_{\mathrm{E}}$ changes, see [194]. After changing this detection angle a camera (not shown) is used to roughly reposition the sample such that the illuminated spot is in focus with the spectrometer. Subsequent XYZ fine-tuning of the sample alignment was done by optimization to the measured emission signal. 

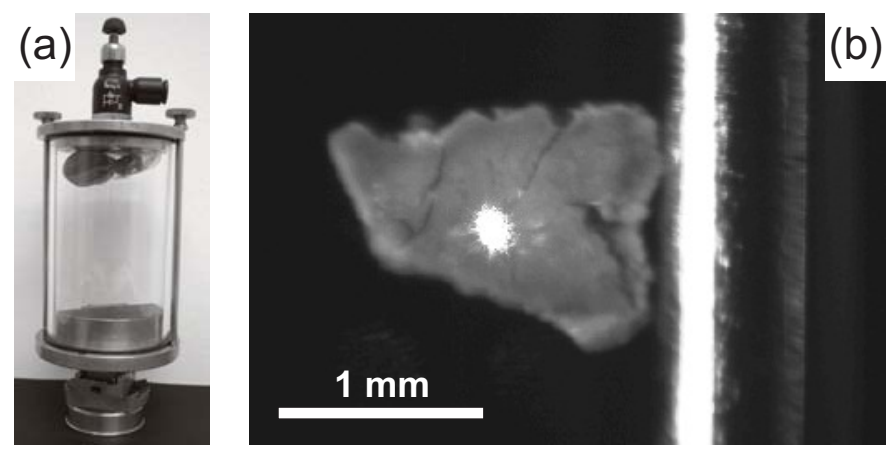

Figure 4.4: (a) Photograph of the vacuum chamber used in the optical experiments. A transparent cylinder is sealed off by two plates. The bottom plate is mounted on four stages and will be fixed to a rotation stage. The top plate contains a valve used to purge the chamber with nitrogen. (b) Front-view, gray-scale image of a titania inverse opal photonic crystal glued to a needle-pin (on the right). The white spot on the sample is a $\lambda=532 \mathrm{~nm}$ laser spot that is used to locally excite PbSe quantum dots inside this structure. Due to a combination of diffusion, scattering and camera exposure time, the size of the laser spot appears much larger than the size of the actual focus.

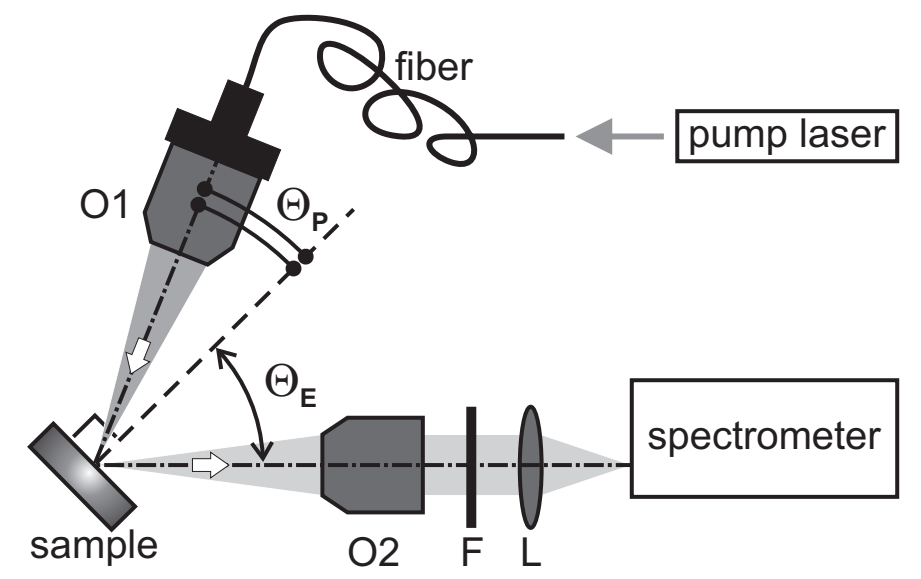

Figure 4.5: Schematic representation of the experiment. Light from a $\lambda=532 \mathrm{~nm}$ pump laser is coupled into a single mode fiber and used to excite quantum dots in a sample, through objective O1. Emission from the dots is collected with objective $\mathrm{O} 2$ and sent to a spectrometer. A longpass filter $(F)$ blocks the laser light. An achromat lens $(L)$ focuses the collected light onto the entrance slit of the spectrometer. The sample is rotated together with the pump beam such that angle $\Theta_{\mathrm{P}}$ is fixed and the emission collection angle $\Theta_{\mathrm{E}}$ changes. Consequently, the excitation conditions are the same for every angle $\Theta_{\mathrm{P}}$. The arrows indicate the direction of the light. 

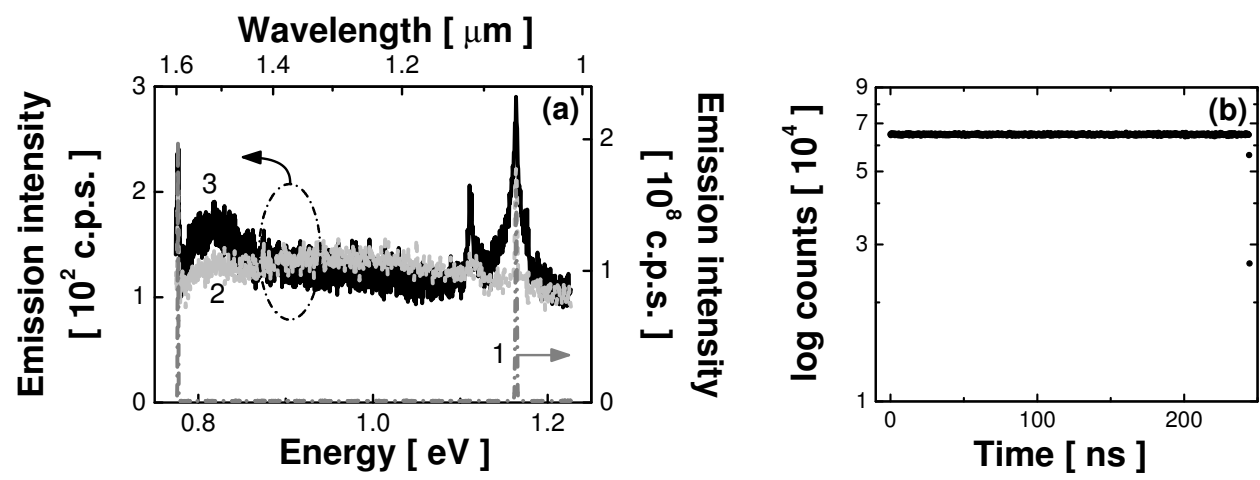

Figure 4.6: Background subtracted spectrographs of a titania inverse opal. (a) Spectrometer signal for different filter settings. Line 1, no filtering, yields two clear peaks at $1.06 \mu \mathrm{m}$ and $1.60 \mu \mathrm{m}$, caused by the second and third order grating mode of the $532 \mathrm{~nm}$ excitation laser. Lines 2 and 3 show the result with one 850 or $1100 \mathrm{~nm}$ long-pass filter in the detection path. Arrows indicate the corresponding vertical axis. Clearly the laser light is strongly suppressed by the long-pass filters. (b) Decay curve histogram measured at $\lambda=1300 \mathrm{~nm}$ shows no sign of any titania emission at short times and is completely determined by the dark signal of the PMT (see Chapter 6 for details). The histogram contains 957 channels of 256 ps.

The current configuration allows the emission detection over an unmatched range of both positive and negative angles.

To reduce systematic errors, the emission detection angle $\Theta_{\mathrm{E}}$ is varied in the following way. First, $\Theta_{\mathrm{E}}$ is increased from $0^{\circ}$ to maximum positive angles in steps of $20^{\circ}$. Subsequently, the angle is decreased in steps of $20^{\circ}$ to maximum negative angles, which results in a data set with steps $\Delta \Theta_{\mathrm{E}}=10^{\circ}$. For some measurements the procedure is repeated to obtain a data set with steps $\Delta \Theta_{\mathrm{E}}=5^{\circ}$. The experiment ends where it started, at $\Theta_{\mathrm{E}}=0^{\circ}$. At the start and end of the experiment the excitation laser is blocked after which the background signal is determined. With this procedure any spectral change in time and changes in the environment can be detected. This provides a useful tool to evaluate the reproducibility of the results.

The sample surface projected onto the entrance slit of the spectrometer changes with $\Theta_{\mathrm{E}}$. The measured intensities are corrected for this. The correction also takes into account the fact that the emission from the sample is peaked around the excitation focus. Appendix D.2 describes the correction in detail.

Emission from the titania backbone exerted influence on the measured signals in earlier experiments at visible wavelengths [30]. An experiment was done to find out if this backbone emission also contributes to the measured signal at $1300 \mathrm{~nm}$. Therefore, a sample was prepared as usual, but without PbSe quantum dots in the hexane. Figure 4.6.(a) shows the measured signals using different long-pass filters in the detection path. For this spectral measurement a relatively large excitation pulse 
energy of $0.53 \mathrm{pJ}$ was used. The peaks at $\lambda=1.06 \mu \mathrm{m}$ and $\lambda=1.60 \mu \mathrm{m}$ are the second and third order grating modes of the excitation laser. The rest of the signal is practically constant over the observed energy range. The small vertical offset from zero intensity can be explained by the drift in the dark-signal of the detector array (see Section 2.4). Figure 4.6.(b) shows the raw output signal from a timeresolved emission experiment on this sample without quantum dots. The signal is completely flat and therefore determined by the dark signal of the photomultiplier. In conclusion, the $850 \mathrm{~nm}$ long-pass filter sufficiently suppressed the laser light, and there is no sign of titania emission. Reasonably, the titania emission is expected from defect luminescence in the middle of the titania band gap, i.e., in the visible, and not around wavelengths $\lambda=1300 \mathrm{~nm}$.

\subsection{Results and discussion}

\subsubsection{Emission spectra and reproducibility}

Two series of experiments were done to verify if the measured emission signals were significant and to find an estimate for the uncertainty in the results. In Figure 4.7 the measured emission signal from PbSe quantum dots inside a non-photonic crystals is compared to PbSe quantum dot emission from a hexane suspension. Both signals look similar, so the signal from the photonic crystal stems mainly from the PbSe quantum dots. In addition, no emission peak was observed from quantum dot free crystals (see Figure 4.6). The relative linewidth increased from $\Delta \lambda / \lambda=0.098$ in the hexane suspension to $\Delta \lambda / \lambda=0.147$ in the titania inverse opal. This is a result of inhomogeneous broadening, i.e., different quantum dots in the suspension experience roughly the same chemical environment, whereas in the photonic crystal the environment depends very much on the location of the quantum dot. A small red-shift of the emission maximum is also observed when the quantum dots are in the photonic crystal. This is caused by the change of the chemical environment experienced by the dots.

The reproducibility of the results was also examined. Figure 4.8 contains the results of measurements on four different samples, using two different excitation lasers (wavelengths $\lambda=532$ and $633 \mathrm{~nm}$ ). Each subplot shows the spectral evolution in time. The plots do not show the same spectral time dependence. Often, a broadening of the emission peak is observed at the high energy side of the spectrum (Figures 4.8.(a,b,d)). Figure 4.8.(a) shows an extreme case where also the peak shifted to the blue. There, the excitation was stopped after three measurements, and restarted the next day. Although the spectral shape did not change over night, the emission intensity reduced by a factor of two. Furthermore, the emission intensity started to increase again when the excitation was resumed. This behavior was also reported for CdSe quantum dots [211]. Figure 4.8.(b), excited using $\lambda=633 \mathrm{~nm}$, and 4.8.(d), excited using $\lambda=532 \mathrm{~nm}$, show results that are typical if a spectral change is observed. The spectral broadening is not accompanied by a frequency 


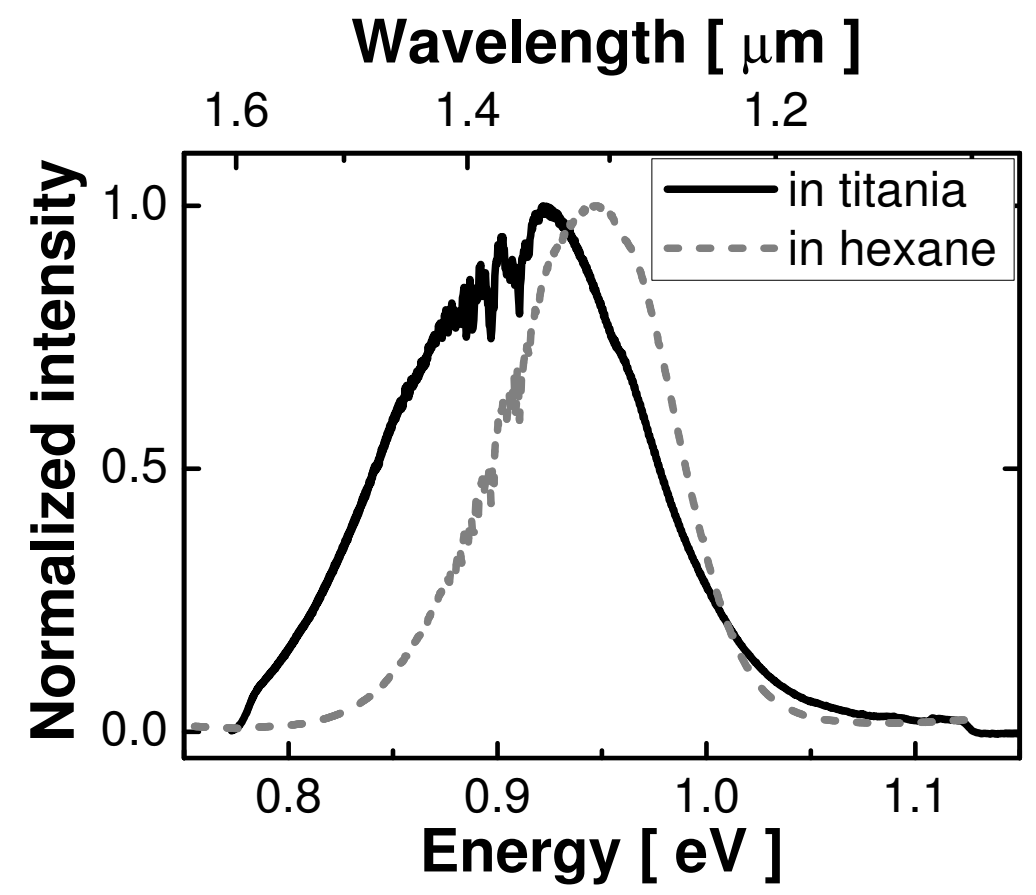

Figure 4.7: Measured emission spectra of PbSe quantum dots in a hexane suspension (dashed line) and inside a non-photonic crystal (solid line). The change in chemical environment experienced by the quantum dot ensemble inside the crystal causes a red-shift and additional inhomogeneous broadening of the spectrum. The rapid signal decrease below $\lambda=1.1 \mu \mathrm{m}$ is due to a long-pass filter. The detector is not sensitive to light with wavelengths $\lambda>1.6 \mu \mathrm{m}$. The fringes around $1.4 \mu \mathrm{m}$ is related to the spectrometer, as discussed in Chapter 2.

shift of the peak, and the red side of the spectrum hardly changes. It is reasonable that the oxidation effects are most strong at the blue side of the spectrum, where the smallest quantum dots emit, as the relative change of these small quantum dots is larger than for larger dots. These two figures show that different excitation lasers can cause the same spectral change. Figure 4.8.(c) shows a rare event where the spectral intensity increases considerably and the peak frequency hardly changes. The small broadening at the red side is very uncommon in our experiments.

It is known that photoluminescence spectra of quantum dots can change. The observed, irreversible blue shift of the dot is caused by photo-oxidation. However, photo-oxidation results in the reduction of the luminescence intensity [212]. Therefore, an additional process should account for the observed intensity increase. Intensity increases with time have been observed before. The origin of this increase is still under debate and, e.g., attributed to rearrangements of surfactant molecules, 


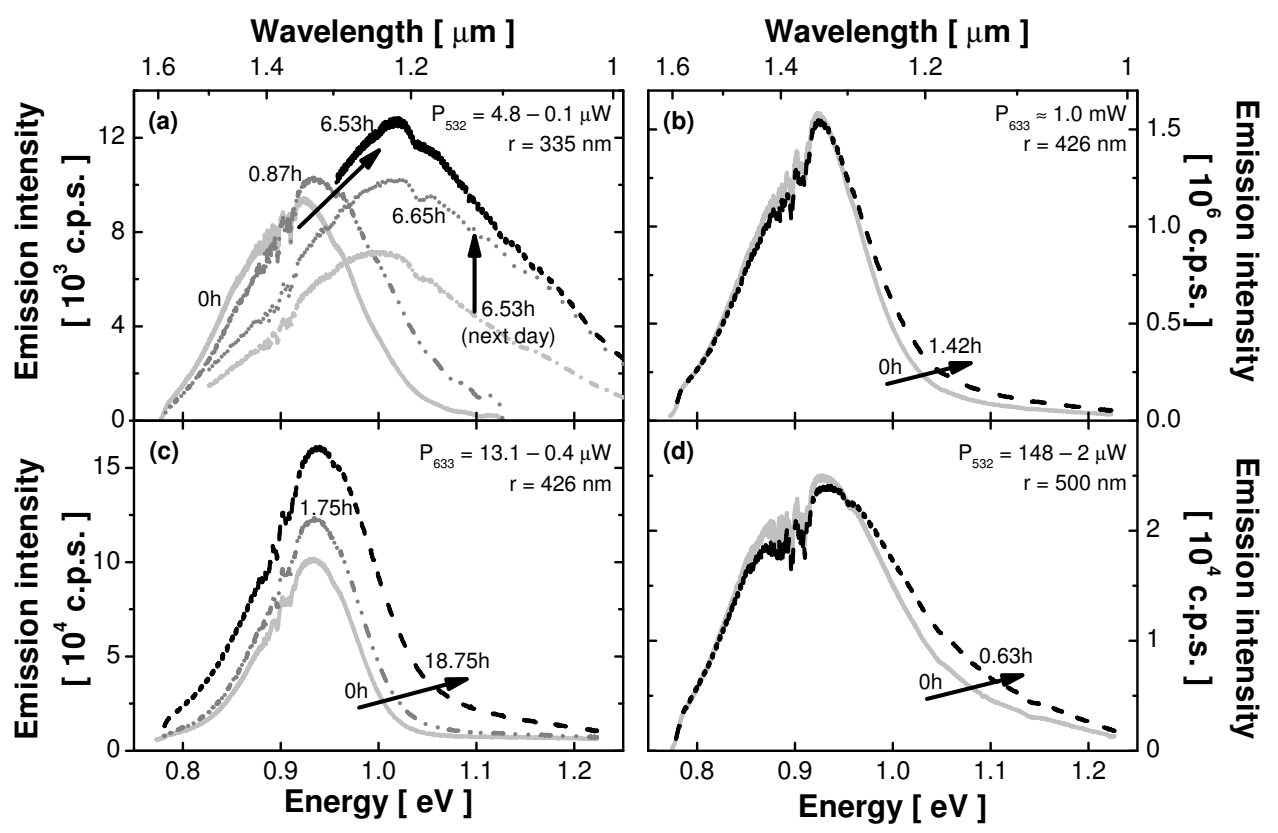

Figure 4.8: Evolution of measured PbSe quantum dot emission spectra from titania inverse opals in time. The labels at the curves indicate the time of excitation with a laser, in hours. Black arrows show the time evolution. The upper right corner of each sub-plot contains the excitation power and wavelength (532 $\mathrm{nm}$ or $633 \mathrm{~nm}$ ), together with the sphere radius used to fabricate the crystal. (a) Emission spectrum broadens, peak shifts to the blue and intensity increases with time. After three measurements the excitation was blocked for 16.5 hours. When the excitation was resumed the emission intensity was twice as low, and increasing. Furthermore, the spectral shape did not change when the excitation was blocked. (b) Hardly any spectral change, using an excitation power much larger than in (a). There is only a slight intensity enhancement at the blue side of the spectrum. The time span is typical for an angle resolved emission experiment. (c) Relatively rare event where the frequency of the peak remains the same while the emission intensity increases considerably. The spectrum broadens at both blue and red side. (d) Only little change at the blue side of the emission spectrum while using relatively high excitation power. The spectral shape looks different from the other measurements due to Bragg diffraction in this photonic crystal. 
photoionization and charge or molecule trapping processes [213-216]. The reproducibility of the intensity increase, after blocking the excitation of the dots for some time, was observed before, see for example [211, 217]. Only recently, PbSe quantum dot that are stable for months were demonstrated [129].

When spectral changes in time are not the interest of study, it is best to try to avoid them. It is common to work in oxygen free environments, or to embed the dots in a photo resist matrix such that at least the oxidation effects are prevented [30, 218]. In the visible range luminescence measurements normally take little time and spectral changes are mostly neglected. Near-infrared experiments are generally more time consuming. Therefore, spectral changes of the quantum dot luminescence should be accounted for. Practically, we need to know if the emission spectrum changed and for which frequency range the change is relevant. Therefore, the measurement procedure was improved by measuring the emission spectrum at the start and end of all experiments. As a result, the reproducibility of the measurement can be judged for all frequencies, and in all experiments. The overlap at the red side of the spectrum, which hardly changes, is very good. For the long integration times used, error bars of $1 \%$ of the experimental value can be used to estimate the uncertainty in the measurement. However, this uncertainty does not account for systematic errors caused by the spectral changes, or alignment issues. When the emission spectrum changes with time, saw-tooth-like shapes appear if the emission intensity is plotted versus the external angle $\Theta_{\mathrm{E}}$. Therefore, the additional uncertainty in the results can be evaluated after a measurement.

\subsubsection{Angle-resolved emission measurements}

To verify that the quantum dots are really inside the photonic crystal, a nonphotonic sample was tested. In this case, the lattice parameter is too small to cause Bragg diffraction at the quantum dot emission wavelength. Figure 4.9.(a) shows the result of the angle resolved emission experiment. The background subtracted data are shown as open squares. Subsequently each data point is corrected for the angle dependent collection efficiency. The signal is normalized to its maximum value. The measured intensity profile is the same for positive and negative angles, which is already an indication of the diffusive nature of the emitted light [187]. In Figure 4.9.(b) the data are compared to models for diffuse volume emitters and isotropic surface emitters ${ }^{\mathrm{ii}}$. The data clearly agree with the volume emitter model. This means that the measured quantum dots are really inside the photonic crystal. The contribution from emitters at the samples' surface can be neglected, as the intensity clearly tends to zero at $\Theta_{\mathrm{E}}=90^{\circ}$. Therefore, the infiltration procedure proved successful.

Is the PbSe quantum dot emission intensity influenced by the stop band in the photonic crystal? To solve this matter experiments were done using samples with different lattice parameter. Figure 4.10 shows the normalized emission spectra mea-

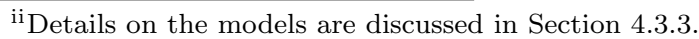



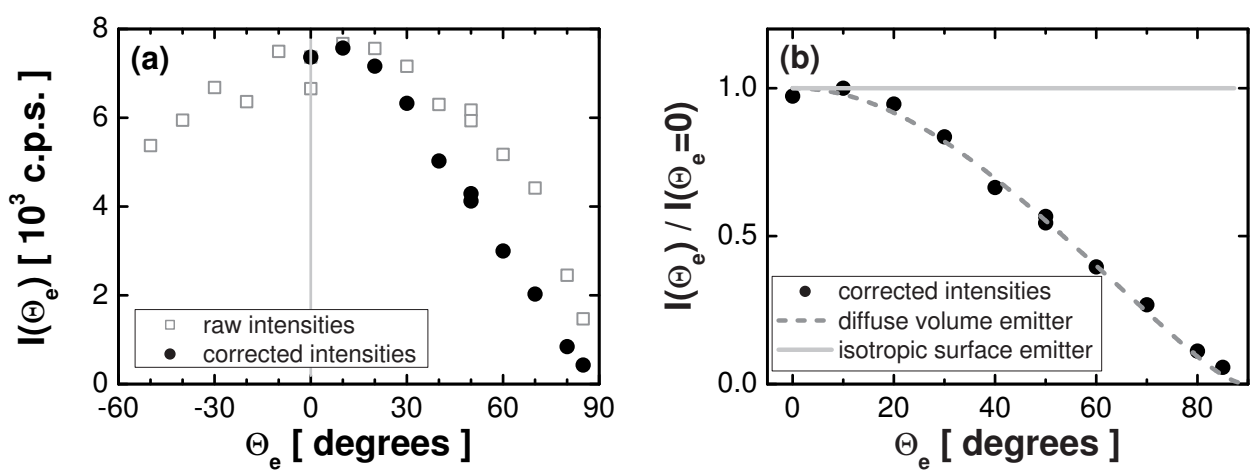

Figure 4.9: Angle-resolved emission intensity of PbSe quantum dots in a nonphotonic titania inverse-opal. The lattice parameter $a=637 \mathrm{~nm}$ and the measurement is done at $\lambda=1400 \mathrm{~nm}$. (a) Open squares are the measured, background subtracted, intensities. Filled dots show the measured data corrected for the detected area. The intensity tends to zero as the angle approaches $\Theta_{\mathrm{e}}=90^{\circ}$. (b) Normalized emission intensity versus detection angle. Filled dots are the same as in (a). The dashed line is the expectation for a diffuse volume emitter. Solid line shows the expected behavior for an isotropic surface emitter.

sured at various angles for samples with two different lattice parameters. Spectral changes with time were determined to be negligible for these experiments. For the non-photonic sample in Figure 4.10.(a), the spectral shape remains the same. A small decrease (increase) at the blue (red) side of the spectrum is observed at $\Theta_{\mathrm{E}}=60^{\circ}$. This deviation from the spectra measured at $\Theta_{\mathrm{E}}=0^{\circ}$, and $20^{\circ}$ is attributed to scattering effects that cause the blue side to be attenuated more efficiently than the red side. Figure 4.10.(b) shows the spectra measured from the photonic sample. The emission intensity is suppressed over a range of frequencies, indicated by the horizontal lines below the spectra. This suppression is caused by the stop band of the photonic crystal. The central wavenumber of the stop band is described by Bragg's law and consequently the band shifts to higher wavenumbers if the measured angle is increased [56]. Correspondingly, the frequency of the emission intensity peak changes with angle. The peak is shifted to the blue if the stop band overlaps the red side of the spectrum, i.e., at small angles. At larger angles the stop band has moved to the blue part of the spectrum and the emission peak is shifted somewhat to the red (not shown). For the largest angle the peak shifts back to the frequency of the dots' actual emission maximum. This experiment demonstrates the influence of the photonic crystals stop bands on the emission spectrum of the quantum dots. This stop band attenuation of a part of the emission spectrum resembles experimental findings using dye molecules, but was not observed with quantum dots before; see Section 4.3.5 for discussion.

The data in Figure 4.10 are only part of a measurements' result. To study the 


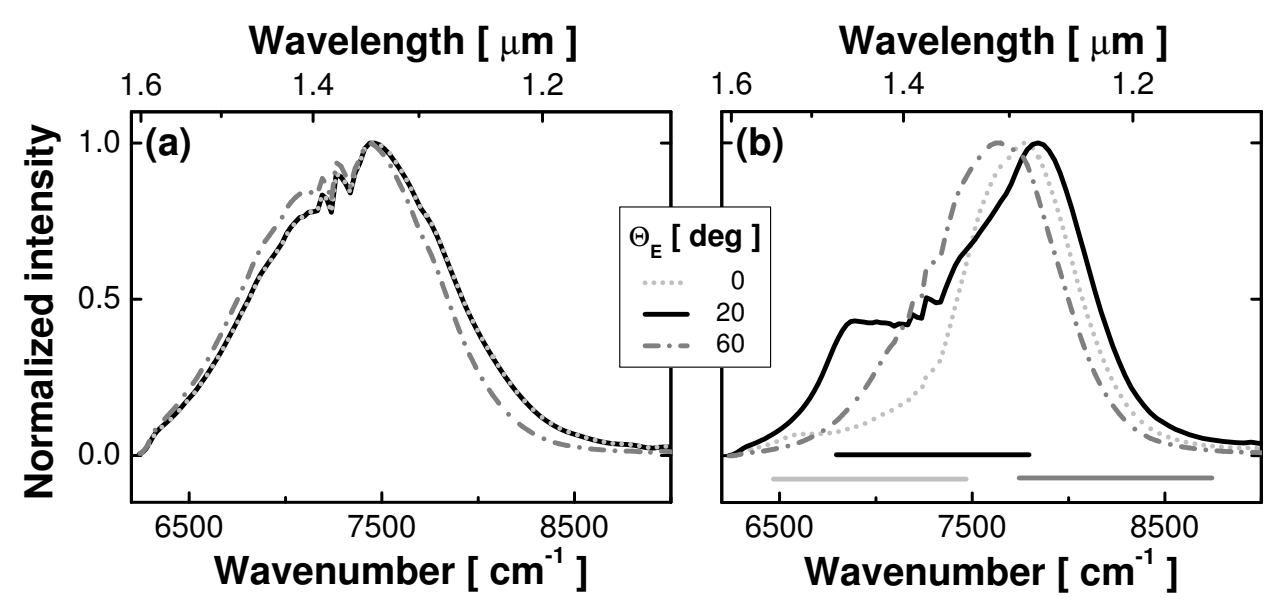

Figure 4.10: Background subtracted and normalized emission spectra, measured at different angles $\Theta_{\mathrm{E}}$. (a) Shows the results for a non-photonic sample with lattice parameter $a=220 \mathrm{~nm}$. There is no sign of a stop band and the spectral shape is practically the same for different angles. (b) Contains the spectra measured from the photonic sample with lattice parameter $a=1114 \mathrm{~nm}$. At $\Theta_{\mathrm{E}}=0^{\circ}$ a stop band appears at $7000 \mathrm{~cm}^{-1}$, that shifts to the blue for larger angles. The frequency range of the stop band is indicated by the horizontal lines below the spectra. The spectra clearly depend on $\Theta_{\mathrm{E}}$, and spectral changes with time were negligible.

angle dependent emission intensity at a certain wavelength it is convenient to plot the data differently. Figure 4.11 shows extensive data sets measured on a nonphotonic and a photonic sample. The non-photonic sample in Figures 4.11.(a-b) does not show any sign of a stop band. The photonic sample in Figures 4.11.(c-d) shows a stop band in the emission intensity. Increase of detection angle results in a shift of the stop band towards shorter wavelengths. To a first approximation this behavior can be explained by Braggs' law.

In Figures 4.11.(b,d) cross sections at constant wavelengths show the angular dependence of the emission intensity. The non-photonic sample shows a Lambertian like emission profile, discussed below. The short wavelength side of this spectrum shows a saw-tooth-like intensity, due to the mentioned spectral changes over time. The rest of the spectrum is hardly affected by this spectral change. The photonic sample behaves very distinctly from a Lambertian emitter. The stop band causes a decrease of the measured emission intensity over an angular range of approximately $40^{\circ}$. A model is used to quantitatively interpret the measurement. This is the topic of the next section. 

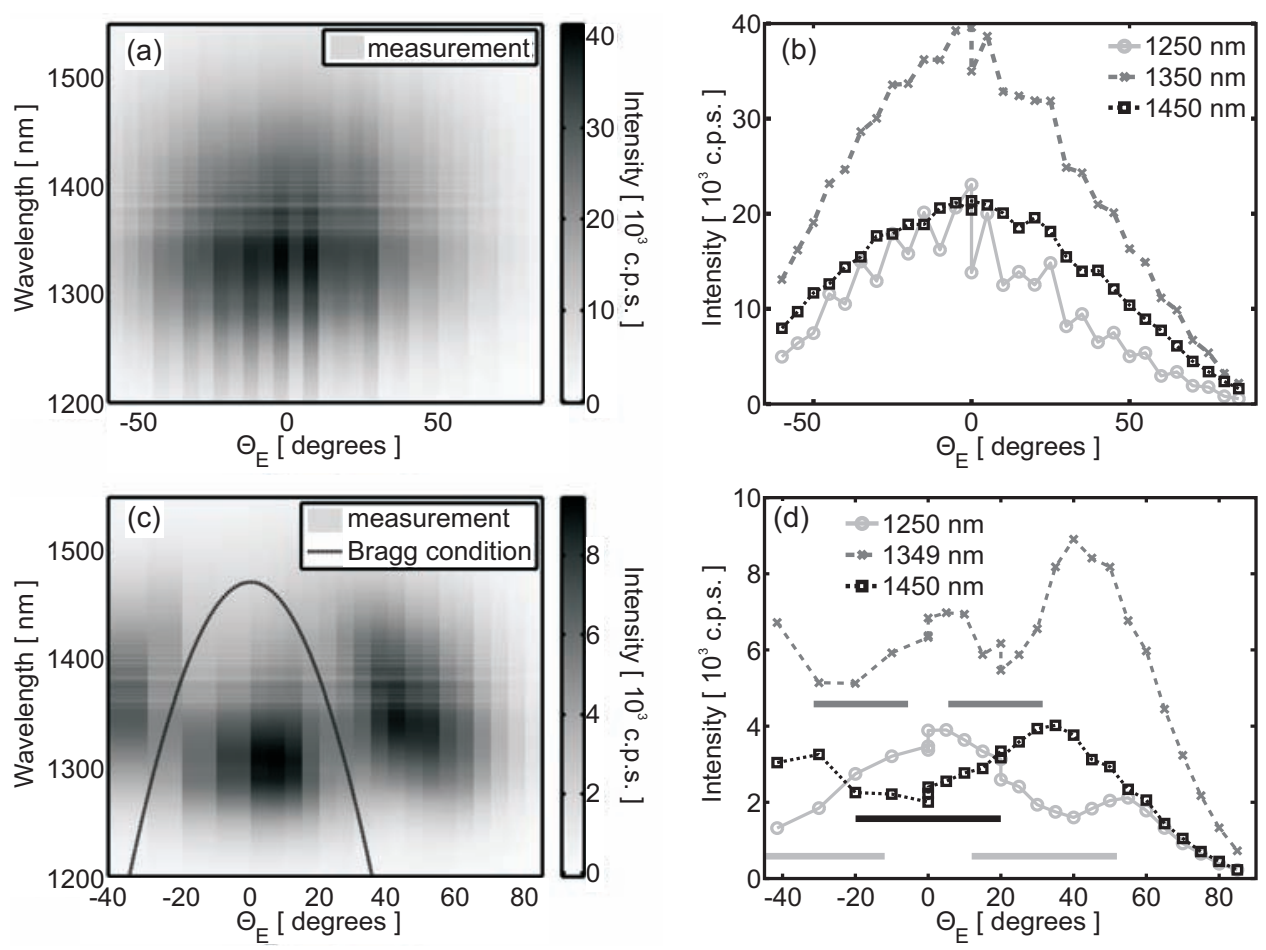

Figure 4.11: Measured, background subtracted and surface corrected PbSe quantum dot emission spectra from titania inverse opals. (a) Emission intensity as a function of wavelength and measured angle $\Theta_{\mathrm{E}}$ for a non-photonic sample. (b) Cross sections of (a) at constant wavelengths: there is no sign of a stop band in either (a) or (b). For $\lambda=1250 \mathrm{~nm}$ the intensity shows a saw-tooth like dependence on the angle. This is due to a time dependent intensity enhancement at the blue side of the emission spectrum. (c) Emission intensity as a function of wavelength and measured angle $\Theta_{\mathrm{E}}$ for a photonic sample. A clear suppression of emission intensity is observed. As an estimate for the wavelength of this attenuation the Bragg condition is plotted (black curve). (d) Cross sections of (c) at specific wavelengths: clear sign of stop bands. Stop band frequencies are indicated with horizontal lines. In (a) and (c) the white areas at wavelengths $\lambda=1200$ and $1550 \mathrm{~nm}$ mark the boundaries of the quantum dot emission spectrum. 


\subsubsection{Expanded escape-function model}

In this section the model to describe the experimental results is defined. We estimate the ratio between the lights' transport mean free path $l$ and a typical sample thickness $L$ to be $l / L \approx 0.3$ for near-infrared frequencies [198]. Therefore, the light becomes diffuse inside the sample. The model is based on diffuse light transmission through multiple scattering, opaque media [176-180, 219]. A complete description is given in References [187, 194, 198]. Here, the most important relations are summarized to explain the essence of this model.

Consider light emission from a sample. The intensity $I\left(\omega ; \mu_{\mathrm{e}}\right)$ that escapes the sample at external angles between $\Theta_{\mathrm{E}}=\cos ^{-1}\left(\mu_{\mathrm{e}}\right)$ and $\cos ^{-1}\left(\mu_{\mathrm{e}}+\mathrm{d} \mu_{\mathrm{e}}\right)$ with respect to the surface normal of the sample is written as

$$
I\left(\omega ; \mu_{\mathrm{e}}\right) \mathrm{d} \mu_{\mathrm{e}}=I_{\text {tot }}(\omega) P\left(\omega ; \mu_{\mathrm{e}}\right) \mathrm{d} \mu_{\mathrm{e}}
$$

Here, $I_{\text {tot }}(\omega)$ is the total spontaneous emission power at frequency $\omega . P\left(\omega ; \mu_{\mathrm{e}}\right)$ is a normalized function that describes the distribution of photons over the available escape angles. We call $P\left(\omega ; \mu_{\mathrm{e}}\right)$ the escape function and use it to compare the model to experimental data. To determine $P\left(\omega ; \mu_{\mathrm{e}}\right)$ experimentally Equation 4.2 is rewritten such that

$$
P\left(\omega ; \mu_{\mathrm{e}}\right)=\frac{I\left(\omega ; \mu_{\mathrm{e}}\right)}{I_{\text {tot }}(\omega)} .
$$

The numerator is given by the measured intensity and the denominator $I_{\text {tot }}(\omega)$ is determined by summing the measured $I\left(\omega ; \mu_{\mathrm{e}}\right)$ weighted by $2 \pi \sin \left(\Theta_{\mathrm{E}}\right) \mathrm{d} \Theta_{\mathrm{E}}$ to approximate the integration over $2 \pi$ solid angle.

For diffuse wave transmission the escape function is analytically derived, and given by $[179,220]$

$$
P\left(\omega ; \mu_{\mathrm{e}}\right)=\frac{3}{2} \mu_{\mathrm{e}}\left[\tau_{\mathrm{e}}(\omega)+\mu_{\mathrm{i}}\right] \cdot\left[1-R_{\mathrm{D}}\left(\omega ; \mu_{\mathrm{i}}\right)\right]
$$

Here, $\cos ^{-1}\left(\mu_{\mathrm{i}}\right)$ is the angle inside the sample, which will be related to $\cos ^{-1}\left(\mu_{\mathrm{e}}\right)$ using Snell's law. $R_{\mathrm{D}}\left(\omega ; \mu_{\mathrm{i}}\right)$ is the angle and frequency dependent internal-reflection coefficient. Finally, $\tau_{\mathrm{e}}(\omega)=z_{\mathrm{e}}(\omega) / l(\omega)$ is the extrapolation length ratio, defined by the extrapolation length $z_{\mathrm{e}}(\omega)$ and the transport mean free path $l(\omega)$ of the sample. $\tau_{\mathrm{e}}(\omega)$ can be expressed as a function of the angle-averaged reflectivity of the sample boundaries $\bar{R}_{\mathrm{D}}(\omega)[178-180]$, i.e.,

$$
\tau_{\mathrm{e}}(\omega)=\frac{2}{3}\left[\frac{1+\bar{R}_{\mathrm{D}}(\omega)}{1-\bar{R}_{\mathrm{D}}(\omega)}\right] .
$$

Furthermore, it was shown how to evaluate this average reflectivity $\bar{R}_{\mathrm{D}}(\omega)$ from the angle and frequency dependent internal reflection coefficient $R_{\mathrm{D}}\left(\omega ; \mu_{\mathrm{i}}\right)[179,180$, 
220]. This results in

$$
\begin{aligned}
\bar{R}_{\mathrm{D}}(\omega) & =\frac{3 C_{2}(\omega)+2 C_{1}(\omega)}{3 C_{2}(\omega)-2 C_{1}(\omega)+2}, \quad \text { with } \\
C_{n}(\omega) & =\int_{0}^{1} R_{\mathrm{D}}\left(\omega ; \mu_{\mathrm{i}}\right) \mu_{\mathrm{i}}^{n} d \mu_{\mathrm{i}} .
\end{aligned}
$$

The escape function from Equation 4.4 can be evaluated if $R_{\mathrm{D}}\left(\omega ; \mu_{\mathrm{i}}\right)$ is known. So far the derivation of the model was general and derived for transport through random, non-photonic, media. This isotropic model for $P\left(\omega ; \mu_{\mathrm{e}}\right)$ successfully described experimental findings when $R_{\mathrm{D}}\left(\omega ; \mu_{\mathrm{i}}\right)$ was modeled using Fresnel's law in combination with an effective refractive index. However, this does not contain the stop bands observed in photonic crystals. Hence, a different approach is needed to find an expression for $R_{\mathrm{D}}\left(\omega ; \mu_{\mathrm{i}}\right)$ and expand the escape-function model to describe photonic crystals.

The reflectivity from titania inverse opals is mainly determined by simultaneous Bragg diffraction from (111) and (200) planes [60]. The internal reflection coefficient is therefore modeled as the sum of two Gaussian reflection peaks [187, 194], i.e.,

$$
R_{\mathrm{D}}\left(\omega ; \mu_{\mathrm{i}}\right)=R_{1}\left(\mu_{\mathrm{i}}\right) \exp \left\{-\frac{\left[\omega-\omega_{1}\left(\mu_{\mathrm{i}}\right)\right]^{2}}{2 \Delta \omega_{1}\left(\mu_{\mathrm{i}}\right)^{2}}\right\}+R_{2}\left(\mu_{\mathrm{i}}\right) \exp \left\{-\frac{\left[\omega-\omega_{2}\left(\mu_{\mathrm{i}}\right)\right]^{2}}{2 \Delta \omega_{2}\left(\mu_{\mathrm{i}}\right)^{2}}\right\},
$$

with angle-dependent peak reflectivities $R_{1,2}\left(\mu_{\mathrm{i}}\right)$, widths $\Delta \omega_{1,2}\left(\mu_{\mathrm{i}}\right)$, and center frequencies $\omega_{1,2}\left(\mu_{\mathrm{i}}\right)$. These center frequencies cannot be described by simple Bragg diffraction, due to many-wave coupling [60]. Therefore, band structure calculations are used to model $\omega_{1,2}\left(\mu_{\mathrm{i}}\right)$. For $R_{1,2}\left(\mu_{\mathrm{i}}\right)$ and $\Delta \omega_{1,2}\left(\mu_{\mathrm{i}}\right)$ normal incidence reflectivity data are used. Furthermore, it is assumed that these four parameters are smooth functions of $\mu_{\mathrm{i}}$. This expanded escape-function model can now be used to describe the results on photonic crystals.

\subsubsection{Comparison experiment with model}

Figure 4.12 shows the escape function $P\left(\omega ; \mu_{\mathrm{e}}\right)$ cross sections measured from two non-photonic samples. Symbols denote measured data and the lines are calculated from the model. There is no stop band. The data at $a / \lambda=0.15$ and 0.58 match the regular escape-function model. The data at $a / \lambda=0.17 \mathrm{~nm}$ deviate from this model, i.e., especially at small angles $\left(\Theta_{\mathrm{E}} \approx 0\right)$ the measured data are above the isotropic model. These data points are indicated by the open squares. At large angles the data are below the model. This deviation is explained by the observed intensity increase at the blue side of the emission spectrum. At $\Theta_{\mathrm{E}}=0^{\circ}$ the result of the very first and last measurement was averaged (see Figure 4.11.(b), $\lambda=1250 \mathrm{~nm}$ for clarity). Due to this intensity increase the normalization used to calculate $I_{\text {tot }}(\omega)$ in Equation 4.3 becomes less accurate. This causes the measurement to deviate from the model. The modulation of measured escape function with external angle 


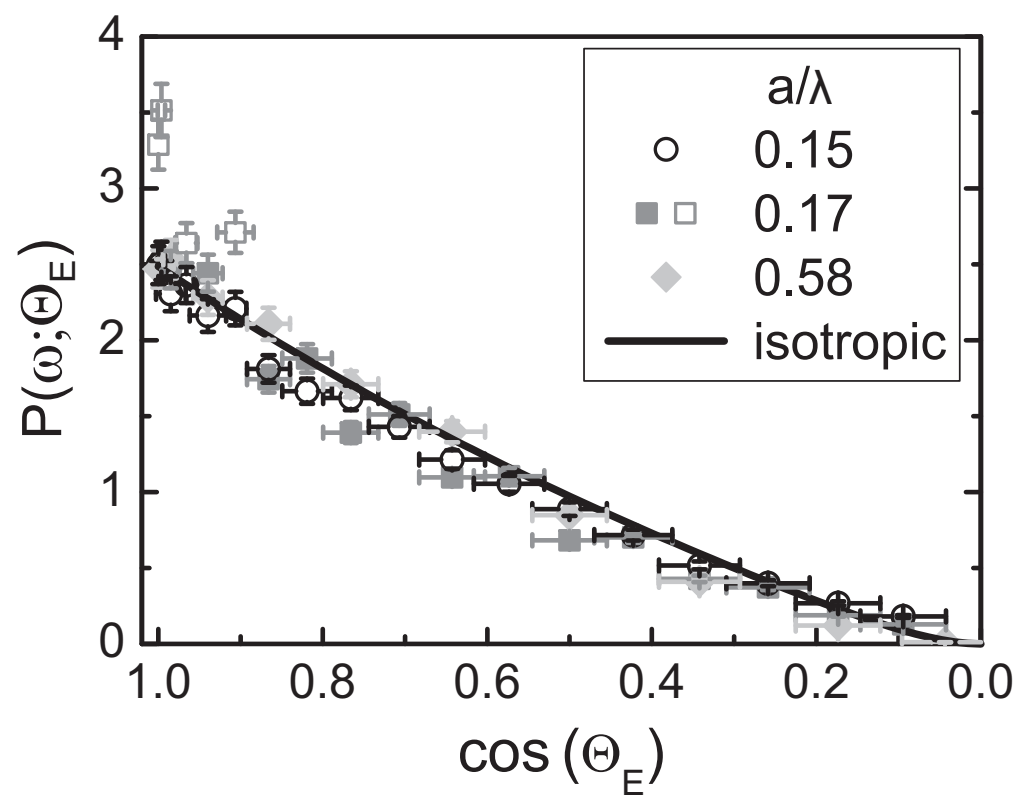

Figure 4.12: Escape functions derived from experiments (symbols) plotted together with the regular escape-function model for isotropic media (solid line). Circles and squares are measured on the same sample with lattice parameter $a=220 \mathrm{~nm}$. The diamonds are data collected from a sample with $a=830 \mathrm{~nm}$, also used in Figure 4.13.(a). Only for $a / \lambda=0.17$ the experimental data deviate from the model (open squares), as a result of an emission intensity increase with time. The regular escape-function model fits the escape-function data from non-photonic titania inverse opals very well over a very large range of reduced frequencies.

is much smaller at the red side of the spectrum. This clearly illustrates the effect of inhomogeneous broadening in the quantum dot ensemble, i.e., the measurements at various wavelengths probe different subsets of quantum dots. Although part of the emission spectrum may change with time the rest of the spectrum is constant.

Figure 4.13 shows the escape function $P\left(\omega ; \mu_{\mathrm{e}}\right)$ over large frequency ranges, derived from experiments and calculated. Figure 4.13.(a) is derived from three experiments on samples with different lattice parameters. The results from the different experiments are separated by the horizontal, gray lines. The central part $(0.7<a / \lambda<0.92)$ shows a strong stop band in the escape probability due to the L-gap. As expected, the frequency of the stop band increases with external angle. An enhanced escape probability is observed at $a / \lambda \approx 0.84$ for $\Theta_{\mathrm{E}} \approx 50^{\circ}$. The enhancement of the escape probability stems from a redistribution of the light that is Bragg diffracted inside the photonic crystal, as discussed below. The data in this central frequency part are the same as in Figure 4.11.(c). At lower frequencies 

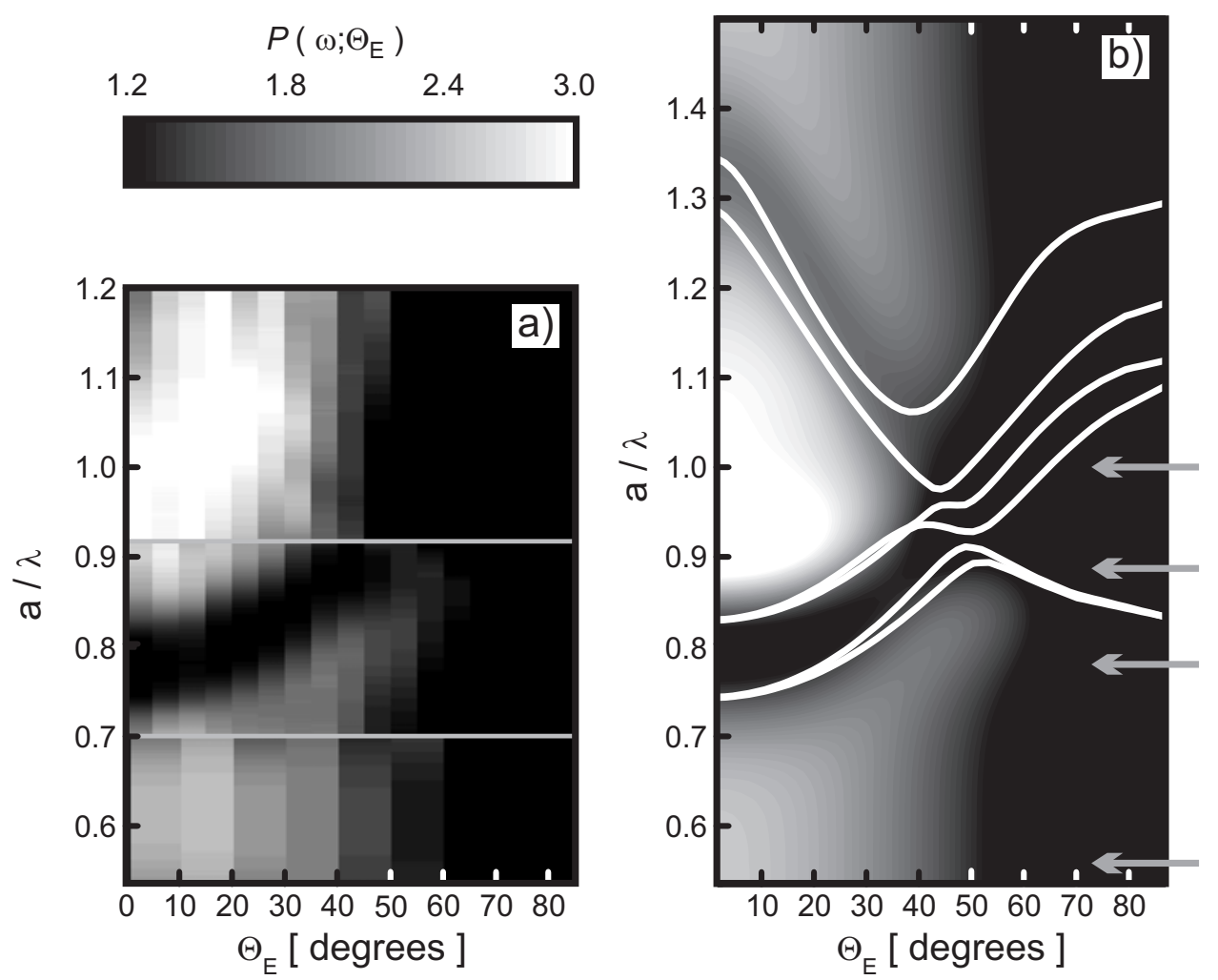

Figure 4.13: Escape probability $P\left(\omega ; \Theta_{\mathrm{E}}\right)$. (a) Experimental results from three data sets, separated by horizontal lines. The low-frequency region shows the expected, Lambertian-like behavior. The central frequency region shows clear inhibition of the escape probability due to Bragg diffraction. Regions of enhanced escape probability are observed around $a / \lambda=0.85$ and 1.0 for $\Theta_{\mathrm{E}} \approx 50^{\circ}$ and $0^{\circ}$ respectively. (b) Result from the expanded escape-function model. For $a / \lambda<1.1$ the model is in accordance with the experimental findings. The stop band caused by Bragg diffraction from (111) lattice planes is observed, together with the regions of enhanced escape-probability. The higher order Bragg wave diffraction is more pronounced in the model. It is recognized by the inhibited escape probability region that starts at $a / \lambda=1.3$ for $\Theta_{\mathrm{E}}=0$ and continues to lower frequencies at larger angles. White curves show the lowest 6 bands along the $L U$ direction, using parallel momentum $\left(k_{\|}\right)$conservation at the surface to transform internal propagation angles into external propagation angles. The horizontal arrows indicate the frequencies of the cross sections used in Figures 4.12 and 4.14. The common gray-scale range is limited for clarity. 
$(a / \lambda<0.7)$ there is no sign of a stop band and the emission escape probability decreases continuously with $\Theta_{\mathrm{E}}$. At high frequencies $(a / \lambda>0.92)$ another region is observed with enhanced escape probability at small $\Theta_{\mathrm{E}}$. The escape probability rapidly decreases at larger angles. Dark areas at the largest frequencies point out the presence of inhibited escape probabilities caused by Bragg-wave-coupling from the (200) lattice planes [187]. These results clearly show the strong interaction between the spontaneously emitted light and the photonic crystal.

Figure 4.13.(b) shows the data from the expanded escape-function model. The calculated escape probability shows the same characteristics as the experimental results. There is a clear inhibition of escape probability starting at $a / \lambda \approx 0.8$, for $\Theta_{\mathrm{E}}=0^{\circ}$. The central frequency of this inhibition shows the same dependence on $\Theta_{\mathrm{E}}$ as the experimental results. Another line of inhibited escape probability runs from $a / \lambda=1.33$ at $\Theta_{\mathrm{E}}=0^{\circ}$ towards $a / \lambda=0.95$ at $\Theta_{\mathrm{E}}=50^{\circ}$. Multiple Bragg-wavecoupling causes the escape probability to be inhibited for angles larger than $40^{\circ}$ and frequencies $0.88<a / \lambda<1.03$. This result agrees well our experimental results. The regions with the measured, enhanced escape probability where also predicted by the model. Clearly, the expanded escape-function adequately describes the experimental data measured from these strongly photonic crystals. The white curves show the lowest $6 \mathrm{LU}$ bands from a band structure calculation [187]. All band energies are multiplied by 1.125 such that the lowest band overlap with the L-gap. The difference between experiments and band structure calculations are not surprising. More information on the titania distribution in these large lattice parameter crystals is needed to verify the model that describes the unit cell. Furthermore, dispersion causes the refractive index to be different in our near-infrared wavelength regime. This first comparison already shows a very good overlap between the calculated band structure and the escape function.

To compare the results from the model and the experiments in more detail, cross sections were made at the frequencies indicated by the arrows in Figure 4.13.(b). Figure 4.14 contains the cross sections in the photonic part of the reduced frequency range. Symbols denote measured data and the lines are calculated from the model. Figure 4.14.(a) shows the escape function versus external angle at three different reduced frequencies $(a / \lambda \in\{0.77,0.87,1.0\})$. The stop band is clearly visible as the region where the escape probability is reduced with respect to the isotropic probability distribution. Outside the stop band the escape probability is higher than the isotropic model. This probability enhancement is caused by a redistribution of the light inside the photonic crystal: for a certain angular range the light Bragg diffracts back into the sample, where it becomes diffuse and escapes the sample at angles away from the Bragg condition. The enhancement is most profound close to the stop band. Further away from the stop band the data converge to the isotropic case.

The model agrees very well with the experimental data. The dash-dotted line shows the regular escape-function model for isotropic emitters. Figure 4.14.(b) represents the same data as Figure 4.14.(a). In contrast, the escape function is now 

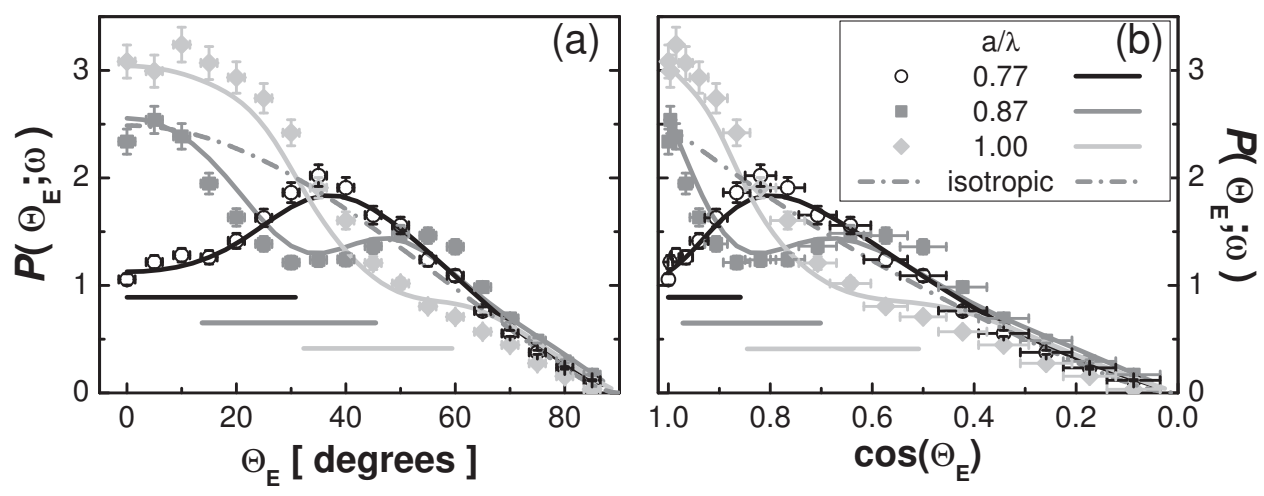

Figure 4.14: Cross sections of escape function at different reduced frequencies $a / \lambda$. Symbols denote the measurement, solid lines result from the expanded escapefunction model, and the dash-dotted line results from the regular escape-function model. (a) Escape function versus external angle $\Theta_{\mathrm{E}}$ shows inhibition and enhancement of the escape probability. Inside (outside) the stop bands the escape probability is inhibited (enhanced) up to $60 \%$ (34\%) with respect to the isotropic distribution. The frequency of the stop band is marked by the horizontal lines below the curves. The stop band moves to higher frequencies at larger angles. (b) Escape function versus $\cos \left(\Theta_{\mathrm{E}}\right)$. This plot contains the same data as in (a), but in this representation the isotropic case tends towards a line. Deviations from this line are easily observed.

plotted versus $\cos \left(\Theta_{\mathrm{E}}\right)$. In this representation the escape function for isotropic emitters tends towards a line, simplifying comparison with experimental results. The experimental data appear even more explicit than the model predicts. Compared to isotropic emission, intensity inhibition as high as $60 \%$ is observed in the photonic crystal. Furthermore, the photonic crystal gave rise to emission intensity enhancements as high as $34 \%$.

The accordance between the experimental results and the model is very good. Furthermore, we would like to stress the importance of these findings for applications at near-infrared frequencies, including the telecom range. Photonic crystals, fabricated simply by self assembly, can be used to concentrate emission from embedded, near-infrared light sources into specific escape angles. This ability to guide light into controlled directions implies a substantial increase in efficiency in applications such as light beaming for the telecom industry, reflector design, and solar cell light control [3]. Furthermore, this guiding can be used to enhance the collection efficiency in single photon on demand applications. 


\subsubsection{Discussion: comparison between results and literature}

Photonic crystals can be divided into two regimes: (1) optically thick samples where the thickness $L$ is at least several times the transport mean free path of light $l$, and (2) optically thin samples where the thickness is about the mean free path of light, or less. In the current chapter we study optically thick samples $(l / L \approx 0.3)$. In these samples light that is Bragg diffracted back into the sample, scatters off defects and becomes diffuse. Subsequently, this light can exit the structure at different angles. This results in the redistribution of the emission intensity described in this chapter. In emission measurements this redistribution causes an emission intensity increase at the edges of the stop band that is clearly not related to LDOS effects; see also [187, 221]. In optically thin samples most emitted light escapes the sample ballistically. There is only little scattering in these structures. Bragg diffraction, however, may still decrease the emission intensity considerably [81].

Strong photoluminescence modifications have been claimed for light sources inside photonic crystals [188, 192, 200-202]. In all cases, the main observation is an attenuation in a small frequency range of the photoluminescence emission spectrum, caused by Bragg diffraction. It was believed that the spontaneous emission properties of the emitter have changed, whereas it was the non-isotropic escape probability that caused the effect. This effect can be understood from Equation 4.2: the emitters properties and the local density of states are contained in $I_{\text {tot }}(\omega)$, whereas the experimentalist measures $I\left(\omega ; \mu_{\mathrm{e}}\right)$. In photonic crystals these spectra cannot be linked without considering the escape function $P\left(\omega ; \mu_{\mathrm{e}}\right)$. Without the escape function it cannot be recognized if light is redistributed over directions that do not show Bragg diffraction. Naturally, lifetime measurements could solve the matter, but were not addressed in these articles.

The escape function from titania inverse opals was also studied using externally injected light [187], and using CdSe quantum dots as embedded emitters in the visible range [194]. A strong redistribution of the light over exit angles was observed, similar to our results. An interesting difference is that in the CdSe quantum dot emission spectra, no stop bands or spectral changes were observed, even though the relative linewidth $\Delta \lambda / \lambda=0.05$. This suggests that the CdSe spectrum is strikingly homogeneously broadened. In contrast, we observe spectral stop bands, similar to earlier work on dyes $[81,82,84,188-194,196]$, in spectra with relative band width $\Delta \lambda / \lambda=0.147$ (see Figure 4.7). The homogeneous line width of PbSe quantum dots is expected to be larger than that of CdSe quantum dots (Chapter 3).

\subsection{Conclusions}

The angular distribution was studied of near-infrared spontaneous emission from PbSe quantum dots inside 3D titania inverse opals. We have observed angular redistribution of the light over the exit angle, caused by a combination of light diffusion and Bragg diffraction in the photonic crystals. The escape function was extracted 
from the experimental data and explained with a diffusion model, expanded to photonic crystals. We found a very good agreement between the measurement and the model. Furthermore, an interpretation of emission enhancement at the sides of the stop bands is given in terms of angular redistribution of emission intensities. In the photonic crystal an escape inhibition of $60 \%$ is observed as well as an enhancement of $34 \%$, both with respect to the escape distribution from non-photonic samples. From these experiments it is clear that the quantum dots are really inside the bulk crystal, which is an important condition for successful lifetime measurements in Chapter 6. Emitters at the surface do not significantly contribute to the signals measured. The data presented form the first experimental evidence of angular redistribution of quantum dot emission from 3D photonic crystals at near-infrared wavelengths, including telecom frequencies. 
Chapter

\section{Time-resolved emission experiments at near-infrared wavelengths}

\subsection{Introduction}

We want to demonstrate spontaneous emission control of light sources in silicon nanophotonic systems, since these are strongly photonic. Therefore, we want to determine the temporal intensity decay at wavelengths $\lambda>1100 \mathrm{~nm}$. At present, almost all time-domain emission measurements are performed by Time Correlated Single Photon Counting (TCSPC). The measurement procedures are described in various books [151, 222, 223]. However, the treatise in these references is always aimed at the visible wavelength range. As our interest is in near-infrared emission, we will examine if the widely used operating procedures are valid for our experiments.

A crucial aspect of TCSPC measurements is the counting statistics. Experimental conditions need to be determined to get sufficient signal to noise ratios together with good counting statistics. These conditions are determined both by the dynamical behavior of the emitters, and by the experimental setup [151, 222, 223]. Unfortunately, the reliability of the counting statistics is rarely discussed in literature.

In this chapter the technical aspects of our TCSPC experiments are introduced. Section 5.2 describes the experimental setup and discusses the counting statistics. Subsequently, Section 5.3 elaborates on the decay curve data analysis. Conclusions form the final part, Section 5.4. 


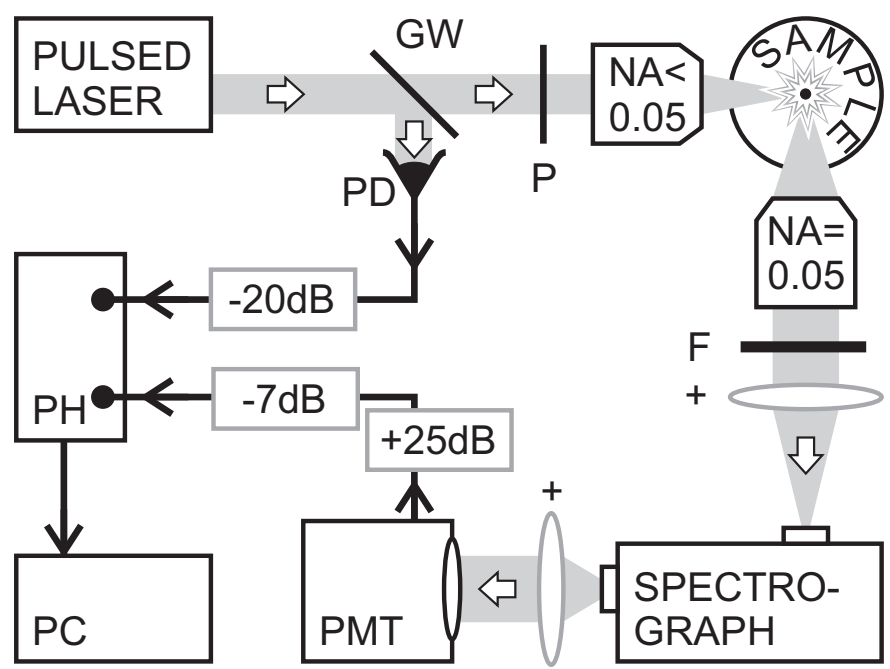

Figure 5.1: Schematic overview of the time correlated single photon counting setup. Gray paths (black lines) denote optical (electrical) signals. Arrows indicate the direction of the signals. The upper right corner shows the light emitting sample inside a nitrogen purged chamber. The abbreviations are: long pass filter $(F)$, glass window $(G W)$, polarizer $(P)$, personal computer $(P C)$, photodiode $(P D)$, PicoHarp300 counting module $(\mathrm{PH})$, and photomultiplier tube (PMT).

\subsection{Time-correlated single-photon counting in the near infrared}

\subsubsection{Experimental setup}

In TCSPC experiments the distribution of emission intensity versus time is determined of excited light sources. This distribution is given by the counts versus time histogram that is constructed by sampling many single photon emission events that each follow an excitation pulse. TCSPC is concerned only with the times of occurrence of events and not with intensities. Therefore, the results are not influenced by intrinsic amplification noise in the detector [222].

Figure 5.1 shows a schematic representation of our TCSPC setup ${ }^{\mathrm{i}}$. A high power laser provides excitation pulses with a time duration of approximately $11 \mathrm{ps}$, and a wavelength $\lambda=532 \mathrm{~nm}$. A Bragg cell reduces the fundamental repetition rate from $82 \mathrm{MHz}$ to 82 or $204 \mathrm{kHz}$, depending on the experiment. A glass window reflects a fraction of the laser light towards a fast photodiode. The diodes' output signal is used as a timing reference, after being attenuated by $20 \mathrm{~dB}$. At the other side of the

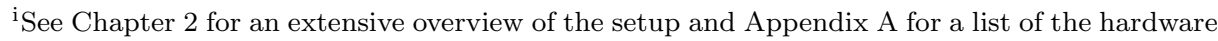
components.
} 
glass window the laser light is attenuated by a polarizer and focused onto a sample using a low NA microscope objective. The sample is positioned inside a chamber and kept under a 0.6 bar nitrogen atmosphere, see Figure 4.4.(a). Light emitted from the sample is collected by a second $0.05 \mathrm{NA}$ microscope objective and guided through a $\lambda=850 \mathrm{~nm}$ long-pass filter. Only light emitted by quantum dots passes the filter and is subsequently focused onto the entrance of a spectrograph. The spectrograph selects light in a small wavelength range using a grating with 85 groves $/ \mathrm{mm}$, blazed for $\lambda=1.35 \mu \mathrm{m}^{\mathrm{ii}}$. This light impinges on a photomultiplier module operated at a supply voltage of $-800 \mathrm{~V}$. The electric output signals of this module is amplified by $18 \mathrm{~dB}$, first by $25 \mathrm{~dB}$ and subsequently attenuated by $7 \mathrm{~dB}$ (Agilent Technologies, 8494A). The electrical signals from the photodiode and photomultiplier module are fed to the synchronized input channels of a real-time processor module. This module accumulates the decay curve histograms and is controlled by a separate computer.

In contrast to earlier TCSPC experiments the processor module is able to measure and store more than one emission event per laser cycle, as long as the events do not occur within the (experimentally determined) $88 \mathrm{~ns}$ dead time of the module. Hence, the peak count rate is in principle limited by the write rate of the module (i.e., $11 \mathrm{MHz}$ ) [223]. In the current setup the practical limitation is set by the maximum anode current of the photomultiplier tube, which results in a maximum count rate of $5 \mathrm{MHz}$.

For decay curve measurements it is essential to know the time resolution of the setup. This can be determined from an instrument response function measurement. Therefore, the laser was used at its fundamental wavelength $(\lambda=1064 \mathrm{~nm})$ and fundamental repetition rate $(82 \mathrm{MHz})$. A fast photodetector was built to generate the reference signal, see Appendix $\mathrm{C}$ for details. Figure 5.2 shows the measured instrument response function. The full width at half maximum is used as the time resolution and determined to be $\Delta t=288 \pm 2 \mathrm{ps}$. This resolution is determined by the transient time spread of the photomultiplier module which is specified to be $0.3 \mathrm{~ns}$. A minimum time resolution of several nanoseconds is needed, as explained in Section 5.3.3. Hence, the setup is suitable for decay curve measurements of $\mathrm{PbSe}$ quantum dot emission.

\subsubsection{Single-photon counting statistics}

The measured decay curve must represent the fluorescence intensity of the light source at time $t_{i}$. Therefore, the number of detected pulses $N_{\mathrm{D}}$ needs to be much smaller than the number of excitations $N_{\mathrm{E}}$, i.e. [224],

$$
F_{\mathrm{D}}=\frac{N_{\mathrm{D}}}{N_{\mathrm{E}}} \ll 1 .
$$

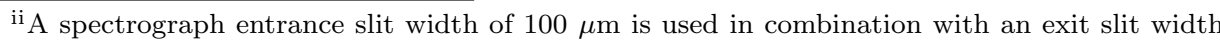
of $20 \mu \mathrm{m}$. This yields a measured spectral resolution of $1.4 \mathrm{~nm}$ (full width half maximum) at $1374 \mathrm{~nm}$.
} 


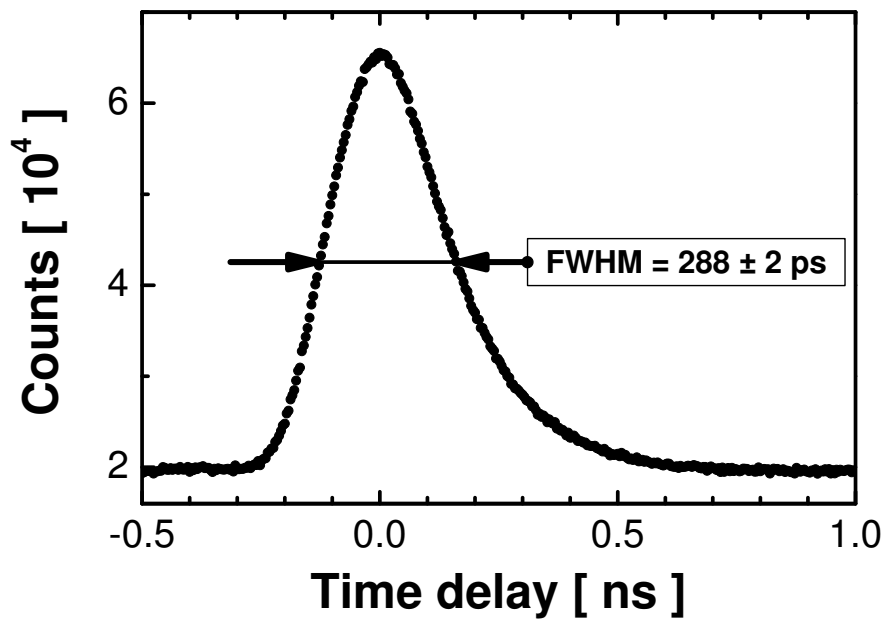

Figure 5.2: Measured instrument response to $\lambda=1064 \mathrm{~nm}$ optical pulses of $11 \mathrm{ps}$ pulse duration (raw data). The system response full width half maximum yields a timing resolution of $288 \pm 2 \mathrm{ps}$. The dark signal of the photomultiplier module causes the background of approximately $2 \cdot 10^{4}$ counts.

In the visible wavelength range, most workers adopt ratios of about $F_{\mathrm{D}}<0.02$, whereas ratios in the region of 0.001 or lower are preferred when pulse pile-up distortions are to be avoided [222]. However, these small ratios are impossible in near-infrared experiments, as the dark signal counting rate is typically larger or approximately equal to the excitation rate. In this case $N_{\mathrm{D}}$ is practically determined by dark signal, and not a useful number to compare to the number of excitations. At near-infrared wavelengths it is useful to find the ratio $F_{\text {NIR }}$ between the number of detected quantum dot emission events $N_{\mathrm{QDE}}$ and the number of excitation pulses $N_{\mathrm{E}}$ : since the dark signal is completely random in time, it results in a flat background in the time correlated results. The average background $\langle B\rangle$ can be subtracted from the measured data set $M\left(t_{i}\right)$ to obtain $N_{\mathrm{QDE}}$. Hence, $F_{\mathrm{NIR}}$ is defined as

$$
F_{\mathrm{NIR}}=\frac{N_{\mathrm{QDE}}}{N_{\mathrm{E}}}=\frac{\sum_{i}\left(M\left(t_{i}\right)-\langle B\rangle\right)}{N_{\mathrm{E}}},
$$

where index $i$ runs over all time bins. The suggested ratios $F_{\mathrm{D}}$ were derived under the assumption that maximally one photon can be detected per excitation pulse. This assumption does not hold for modern setups. Consequently, a new functional upper limit for $F_{\mathrm{NIR}}$ needs to be determined.

For good signal to noise ratios $F_{\text {NIR }}$ should be as large as possible. However, too large values will cause the mentioned photon pile-up. This pile-up effect is illustrated in Figure 5.3 on time-resolved emission from PbSe quantum dots suspended in hexane. To change $F_{\mathrm{NIR}}$ the excitation power is varied at a constant repetition 

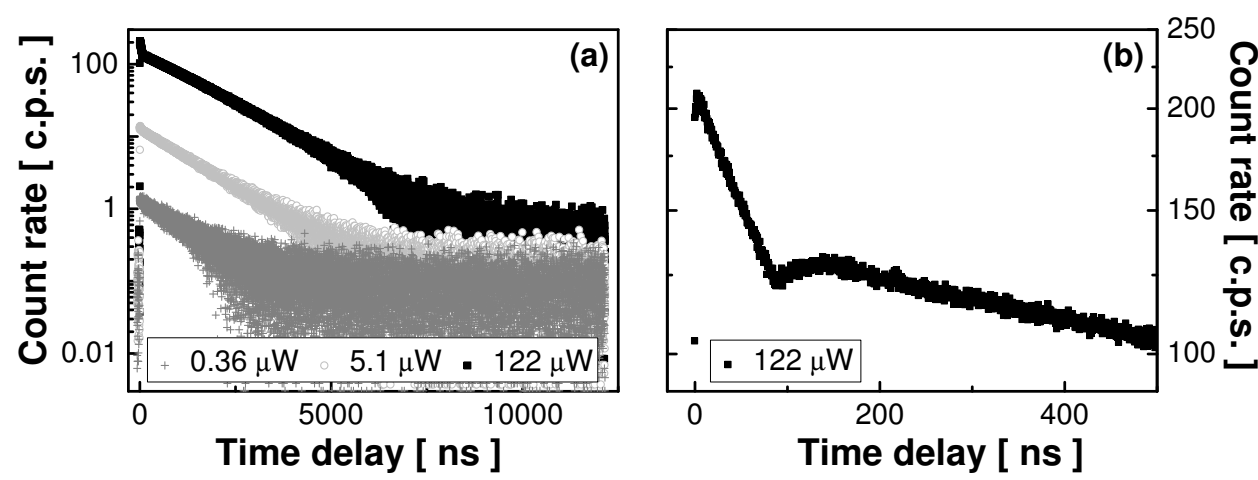

Figure 5.3: Count rate versus time histogram of a $9.2 \cdot 10^{-6} \mathrm{M}$ PbSe quantum dots in hexane, measured at wavelength $\lambda=1300 \mathrm{~nm}$. An average background signal of approximately 8.4 c.p.s. has been subtracted for each time channel. (a) Count rates measured with different excitation powers, i.e., $0.36 \mu W$ (gray plus signs), $5.1 \mu W$ (light gray, open circles), and $122 \mu W$ (black squares). Both signal to noise ratio and count rate go up with excitation power. (b) Zoom-in on the decay curve measured with the highest excitation power. At time delays below 88 ns the slope is very steep; a clear indication of photon pile-up. At longer time delays, the counting statistics improves and the signal starts to recover.

rate of $82 \mathrm{kHz}$. Figure 5.3.(a) shows how the background subtracted count rate and signal to noise ratio increase with excitation power. Figure 5.3.(b) shows a zoomin of the peak count-rate measured with the maximum excitation power. Photon pile-up causes a steep decrease in count rates during the first $88 \mathrm{~ns}$. As mentioned this time range corresponds to the dead time of the counting module. After $88 \mathrm{~ns}$ the count rate increases, which is unphysical in spontaneous emission decay curves. Measurements can be corrected for pile-up when a-priori knowledge of the decay curves' shape is available [223]. These shapes are not known and therefore pile-up effects should be prevented in the intended experiments.

The upper limit for $F_{\mathrm{NIR}}$ is determined by the dynamical behavior of the system of interest and, therefore, determined by measuring PbSe quantum dot emission from inverse opals. The background subtracted data were modeled to a log-normal distribution of decay rates (see Section 5.3.4). The $\chi_{\text {red }}^{2}$ is calculated as a measure for the goodness of fit (see Section 5.3.2). If the model describes the experimental data well, the goodness of fit should be $\chi_{\text {red }}^{2}=1$. In Figure 5.4 the goodness of fit is plotted versus $F_{\mathrm{NIR}}$. If $F_{\mathrm{NIR}}$ exceeds $1 \%$ the goodness of fit deviates significantly from $\chi_{\text {red }}^{2}=1$. For $F_{\mathrm{NIR}}$ less than $1 \%$ we observe $\chi_{\text {red }}^{2} \approx 1$ and therefore the selected model is appropriate to describe the decay curves. To prevent experimental deficiencies, like pile up, $F_{\mathrm{NIR}}<1 \%$ is used in the time-resolved quantum dot emission experiments from titania inverse opals. Hence, we found an experimental boundary condition: no counting deficiencies occur if the detected 


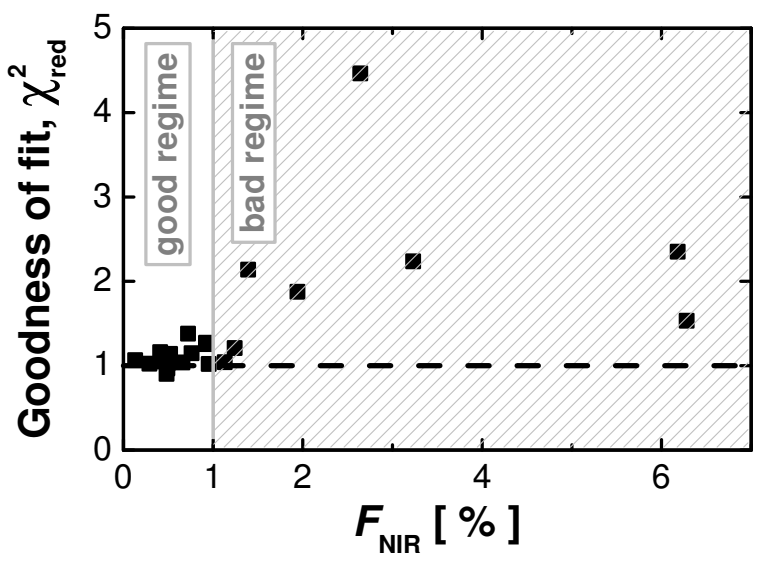

Figure 5.4: Goodness of fit $\chi_{\text {red }}^{2}$ versus the ratio between the number of emission events and the number of excitation pulses, i.e., $F_{\text {NIR }}$. Results from a log-normal decay rate distribution model used to describe PbSe quantum dot emission from titania inverse opals. The horizontal, dashed line is the expected result if the fit is optimal $\left(\chi_{\mathrm{red}}^{2}=1\right)$. The hatched area for $F_{\mathrm{NIR}}>1 \%$ marks the region where the model does not fit the experimental data. This region is to be avoided in subsequent experiments.

quantum dot emission rate is kept below $1 \%$ of the excitation rate. Use of this important condition guarantees good counting statistics. Therefore, the measured decay curves truly represent the fluorescence intensity of the light source in time, and we are ready to analyze the data.

\subsection{Data analysis}

This section discusses the technical details needed to process and model the decaycurve data. First the signal background corrections are discussed. Then the goodness of fit is introduced and the useful data ranges are determined. Subsequently, a decay-rate-distribution model to describe the data is selected, and the corresponding parameter dependence is discussed. Finally, the average and variance of this distribution is defined.

\subsubsection{Raw decay-curve measurement}

The first thing we want to know is what a measured decay curve signal looks like. Figure 5.5.(a) shows the result from a TCSPC experiment with PbSe quantum dots inside a titania inverse opal photonic crystal. At short time delays quantum dot decay is observed. After about $200 \mathrm{~ns}$, the measured signal is nearly constant, at a remarkably high value of $7.3 \cdot 10^{4}$ counts. This large background value is typical 


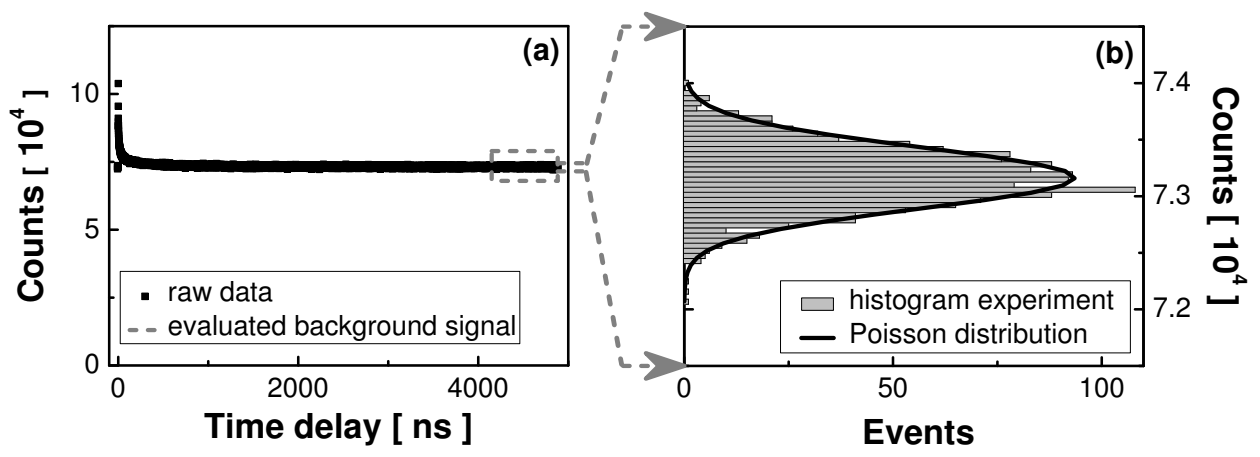

Figure 5.5: Spontaneous emission decay of colloidal PbSe quantum dots inside a non-photonic, titania inverse opal with lattice parameter $a=220 \mathrm{~nm}$. (a) Raw data: 9552 time bins of 0.512 ns. Quantum dot decay is only visible during the first 200 ns. The high, horizontal background level is caused by the dark signal of the detector. (b) Histogram of the number of detected counts (bars) per time bin extracted from the last $15 \%$ of the data, i.e., background signal. The solid line is the Poisson distribution determined by the mean of the histogram. The data was collected in one hour, at a wavelength $\lambda=1300 \mathrm{~nm}$, and using a repetition rate of $204 \mathrm{kHz}$.

for TCSPC experiments in the near infrared and stems from the detectors' dark signal. For comparison: the photomultiplier module used typically has 2000 times more dark signal and a 20 times lower quantum efficiency as compared to modules that work in the visible (e.g., the Hamamatsu H7421-40). Figure 5.5.(b) contains a histogram of the background signal which reveals the expected Poisson distribution in the number of detected counts per bin. Clearly, the dark signal constitutes the major part of the measured counts in near-infrared TCSPC experiments using photomultipliers. However, as the dark signal results in a flat background in the time correlated results, it can easily be accounted for.

The quantum dot decay curve appears after subtraction of the average background from the measured data. Figure 5.6 shows the decay curves for PbSe quantum dots in different environments. Figure 5.6.(a) contains two decay curves measured from quantum dots inside titania inverse opals with different lattice parameters. Both curves show clear non-exponential behavior caused by the photonic crystal. Furthermore, the decay curves display different decay rates due to differences in the density of states between the two crystals (see Chapter 6). Figure 5.6.(b) shows a decay curve of PbSe quantum dots inside a hexane suspension. In contrast to the PbSe quantum dot emission from inverse opals, single exponential decay is observed from this hexane suspension. The corresponding decay rate can be used to estimate the oscillator strength, as discussed in Chapter 3. Furthermore, this figure shows how the averaging over subsequent time bins makes the decay curve 

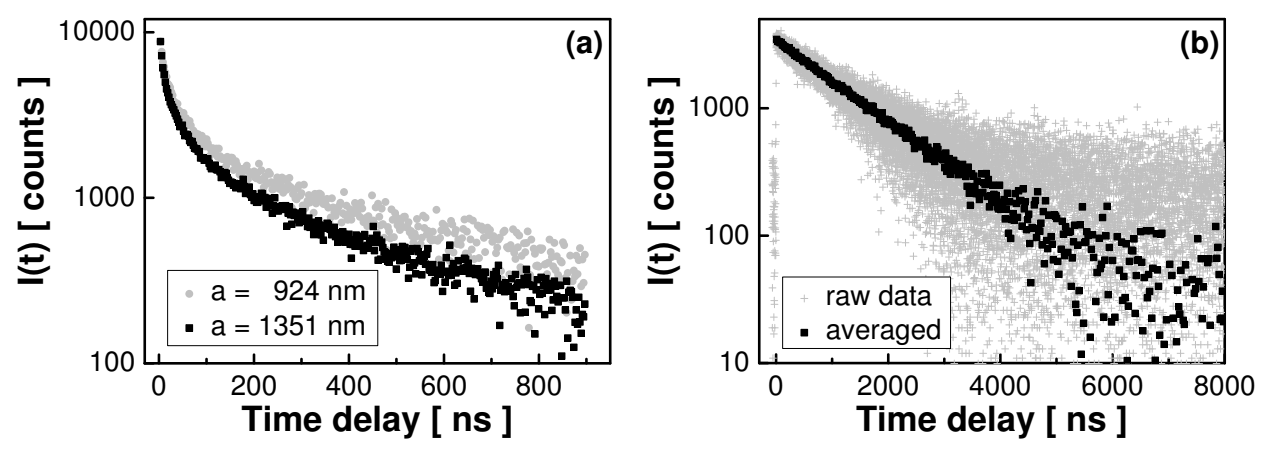

Figure 5.6: Decay curve histograms after background subtraction and time rebinning. (a) PbSe quantum dots inside titania inverse opals with lattice parameters $a=924 \mathrm{~nm}$ (gray circles) and $a=1351 \mathrm{~nm}$ (black squares). The second data set is multiplied by 0.533 to overlap the first data set at zero time delay. In these photonic crystals the decay curves are clearly non-exponential. The fastest decay is measured from the sample with the largest lattice parameter. (b) PbSe quantum dots in a hexane suspension. The decay is single exponential. The black squares are obtained by averaging over 39 points of the raw data set. Due to this averaging, clearly, the decay becomes more distinct. All curves were measured at a wavelength $\lambda=1300 \mathrm{~nm}$.

more pronounced.

The near-infrared decay curve measurement and background correction are resolved. The next step is to find an appropriate model to describe the data, such that a solid comparison can be made between different measured decay curves. In order to choose a model a measure for the goodness of a fit is needed. This is the subject of the following section.

\subsubsection{Evaluation of goodness of fit: use $\chi_{\text {red }}^{2}$}

To quantify the goodness of a model, the reduced $\chi^{2}$ is a suitable parameter. In the course of our work, we have come to identify a widely made error in calculating $\chi_{\text {red }}^{2}$, which is very relevant to experiments with a high dark count (such as here in the near infrared) but is easy to overlook for a low dark count (typical for the visible range).

The $\chi_{\text {red }}^{2}$ is defined as [225]:

$$
\begin{aligned}
\chi_{\text {red }}^{2} & =\frac{1}{N-p} \chi^{2} \\
& =\frac{1}{N-p} \sum_{i=1}^{N} \frac{\left(D_{f}\left(t_{i}\right)-F\left(t_{i}\right)\right)^{2}}{\sigma_{D_{f}}^{2}\left(t_{i}\right)},
\end{aligned}
$$


where $t_{i}$ was chosen as the running parameter, for later convenience. $N$ is the number of data points, $p$ is the number of adjustable parameters, $D_{f}\left(t_{i}\right)$ is the measured data in the $i^{t h}$ time bin, $F\left(t_{i}\right)$ is the corresponding value from the model, and $\sigma_{D_{f}}^{2}\left(t_{i}\right)$ is the variance of the datum at time $t_{i}$. If a model fits the data well, Equation 5.4 should result in $\chi_{\text {red }}^{2}=1$.

For data with Poisson noise the variances are equal to the average (mean) measured values $\left\langle D_{f}\left(t_{i}\right)\right\rangle$. Because a decay curve measurement is very time-consuming the experiment cannot be repeated over and over again to determine $\left\langle D_{f}\left(t_{i}\right)\right\rangle$. Therefore, it is assumed that the mean value of the number of counts in time bin $t_{i}$ is well approximated by the measured number of counts in $t_{i}$ determined by only one experiment, i.e.,

$$
\sigma_{D_{f}}^{2}\left(t_{i}\right)=\left\langle D_{f}\left(t_{i}\right)\right\rangle \approx D_{f}\left(t_{i}\right)
$$

With Equation $5.5 \chi_{\text {red }}^{2}$ can be written in the commonly used form,

$$
\chi_{\text {red }}^{2} \approx \frac{1}{N-p} \sum_{i=1}^{N} \frac{\left(D_{f}\left(t_{i}\right)-F\left(t_{i}\right)\right)^{2}}{D_{f}\left(t_{i}\right)} .
$$

Erroneous results occur if Equation 5.6 is applied to background subtracted or time-averaged data sets. Consider a data set $D_{f}\left(t_{i}\right)$ that is obtained after the subtraction of an average background $\langle B\rangle$ from the raw measurement data $M\left(t_{i}\right)$. The variance of $D_{f}$ is ${ }^{\text {iii }}$

$$
\begin{aligned}
\sigma_{D_{f}}^{2}\left(t_{i}\right) & =\operatorname{var}\left[M\left(t_{i}\right)-\langle B\rangle\right] \\
& =\operatorname{var}\left[M\left(t_{i}\right)\right]+\operatorname{var}[\langle B\rangle] \\
& =\left\langle M\left(t_{i}\right)\right\rangle+0 \approx M\left(t_{i}\right) \\
& \neq D_{f}\left(t_{i}\right) .
\end{aligned}
$$

Hence, Equations 5.5 and 5.6 do not hold.

Additional averaging of $M\left(t_{i}\right)$ over $T$ successive data points results in a reduced set of points $M_{r b}\left(t_{j}\right)$, where the subscript " $r b$ " denotes the re-binning. Although the mean value of both data sets are the same, the variance of the latter is reduced by a factor $T$, i.e.

$$
\operatorname{var}\left[M_{r b}\left(t_{j}\right)\right]=\frac{\left\langle M_{r b}\left(t_{j}\right)\right\rangle}{T},
$$

where $j$ denotes the $j^{\text {th }}$ set of points that was averaged over.

After substitution of Equations 5.9 and 5.11 into Equation 5.4 we find the correct expression to calculate $\chi_{\text {red }}^{2}$ for a background-subtracted and time re-binned data set:

$$
\chi_{\mathrm{red}}^{2} \approx \frac{T}{N_{r b}-p} \sum_{i=1}^{N_{r b}} \frac{\left(M_{r b}(i)-\langle B\rangle-F(i)\right)^{2}}{M_{r b}(i)},
$$

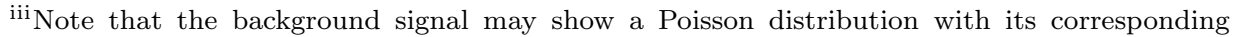
variance, whereas the variance of its mean value is of course zero, as used in Equation 5.9.
} 

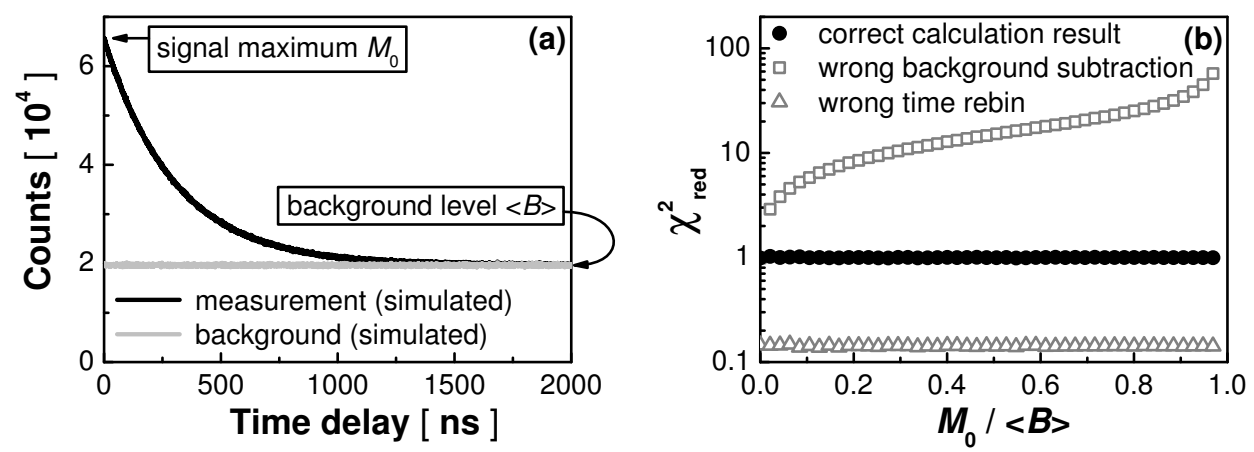

Figure 5.7: Influence of the variance in calculations of the reduced chi-square $\chi_{\text {red }}^{2}$. (a) Simulated data set that consists of a single exponential decay curve with a lifetime of $300 \mathrm{~ns}$ added to a background signal (black line), and a curve that only contains the background signal (gray line). Both lines contain Poisson noise. " $M_{0}$ " is the maximum number of counts obtained in a single bin, and " $\langle B\rangle$ " is the average background number of counts, here $\langle B\rangle=0.3 M_{0}$. The data span a time domain from 2 to $2000 \mathrm{~ns}$, using a bin-width of $256 \mathrm{ps}$. (b) Goodness of fit $\chi_{\text {red }}^{2}$ calculated for three definitions of the variance: 1) the variance is underestimated while the background subtraction is not taken into account correctly (open squares). 2) The variance is overestimated while data rebinning is not taken into account correctly (open triangles). 3) Results for correct background subtraction and data averaging (black dots); clearly this set shows $\chi_{\text {red }}^{2}=1$. To obtain statistically converged results the simulation is repeated 2000 times. To guarantee that the fitted data set shows single exponential decay the time domain that is used in the fit is cropped whenever the first $D_{f}\left(t_{i}\right)$ point is smaller than the standard deviation of the background signal.

where $N_{r b}$ is now the number of data points in the new, re-binned data set. Equation 5.12 is used to calculate $\chi_{\text {red }}^{2}$ in subsequent sections. This provides a powerful tool to evaluate fit results and compare different models.

A simulation was done to illustrate the importance of using the correct definition to calculate $\chi_{\text {red }}^{2}$. A single exponential decay curve $D\left(t_{i}\right)=M_{0} e^{-t_{i} / 300}$ is defined with additional Poisson noise. $M_{0}$ is constant and time $t_{i}$ is considered to be in nanoseconds. Subsequently, a background signal $B\left(t_{i}\right)$ with Poisson noise is defined around a certain mean value $\langle B\rangle$. The measured signal $M\left(t_{i}\right)$ is defined as the sum of the decay curve and background signal, i.e.,

$$
M\left(t_{i}\right)=D\left(t_{i}\right)+B\left(t_{i}\right)
$$

See Figure 5.7.(a) for an example of the simulated, measured signal and the corresponding background signal. The average background $\langle B\rangle$ is subtracted from $M\left(t_{i}\right)$ to obtain the data set $D_{f}\left(t_{i}\right)$. In a subsequent simulation the data set is reduced 
by averaging over seven subsequent data points. All data sets are modeled with a single exponential decay curve.

Figure 5.7.(b) shows the calculated values for $\chi_{\text {red }}^{2}$ as a function of the ratio between the signal maximum $M_{0}$ and the average background $\langle B\rangle$. The open symbols denote the erroneous results using Equation 5.6. For the background subtracted data set $\chi_{\text {red }}^{2} \gg 1$ when the average background is larger than $0.5 \%$ of the maximum signal. The simulation on the averaged data set results in $\chi_{\text {red }}^{2}=1 / 7$, which is seven times too low. The closed dots show the correct result $\left(\chi_{\text {red }}^{2}=1\right)$ calculated from Equation 5.12. Clearly, the correct way to calculate $\chi_{\text {red }}^{2}$ is understood. Hence, we can quantify the goodness of different models used to describe our decay curves.

\subsubsection{Time range and bin width used for modeling}

A typical decay curve measurement yields a minimum of $9.57 \cdot 10^{3}$ data points. Practically, this number is too large to calculate the model-parameters to describe the data. Here, two ways to reduce the data set are examined: 1) use time averaging, i.e., adjacent time bins are grouped as a single time bin with the average number of counts and an increased time bin width. 2) Use a time cut-off $t_{\mathrm{C}}$ and disregard data measured at times $t>t_{\mathrm{C}}$. A log-normal distribution of decay rates is used to model the reduced data sets (see Section 5.3.4 for details). Subsequently, the influence of the data set reduction is evaluated by comparing the outcome of the model parameters $\left(\gamma_{\mathrm{mf}}\right.$, width, and scale $)$ for different data sets.

Figures 5.8.(a to c) contain the model parameter results as a function of time bin width $\Delta t$. The parameter values reproduce as long as $\Delta t \leq 7 \mathrm{~ns}$. For $\Delta t>7 \mathrm{~ns}$ the fit results deviate and the uncertainties in the parameters increases. For further experiments the averaging is kept on the safe side, using $\Delta t=2.56 \mathrm{~ns}$, which reduces the data set by a factor of 5 .

Figures 5.8.(d to f) show the fit parameter results as a function of the time cut-off $t_{\mathrm{C}}$. For $t_{\mathrm{C}}<100 \mathrm{~ns}$ the parameters values show large uncertainties. At $t_{\mathrm{C}}=400 \mathrm{~ns}$ the quantum dot emission signal becomes smaller than the standard deviation of the background signal. For larger times the parameter results start to change and, therefore, $t_{\mathrm{C}}=400 \mathrm{~ns}$ is chosen as time cut-off. As the time bin width is increased in subsequent measurements the standard deviation in the data reduces and the value for $t_{\mathrm{C}}$ may be increased. The used value is kept constant for all measurements at $t_{\mathrm{C}}=900.096 \mathrm{~ns}$. This cut-off results in a data set reduction of $82 \%$. Together with the 5 times reduction from the time-averaging process the raw data set may thus be reduced by $96 \%$ without restriction. This reduction leads to tremendous calculation time savings, and the data can now easily be processed.

\subsubsection{Selection of decay-curve model}

It is a general problem to describe relaxation processes which do not follow a simple single-exponential model [226]. Currently, no non-exponential model can be an- 

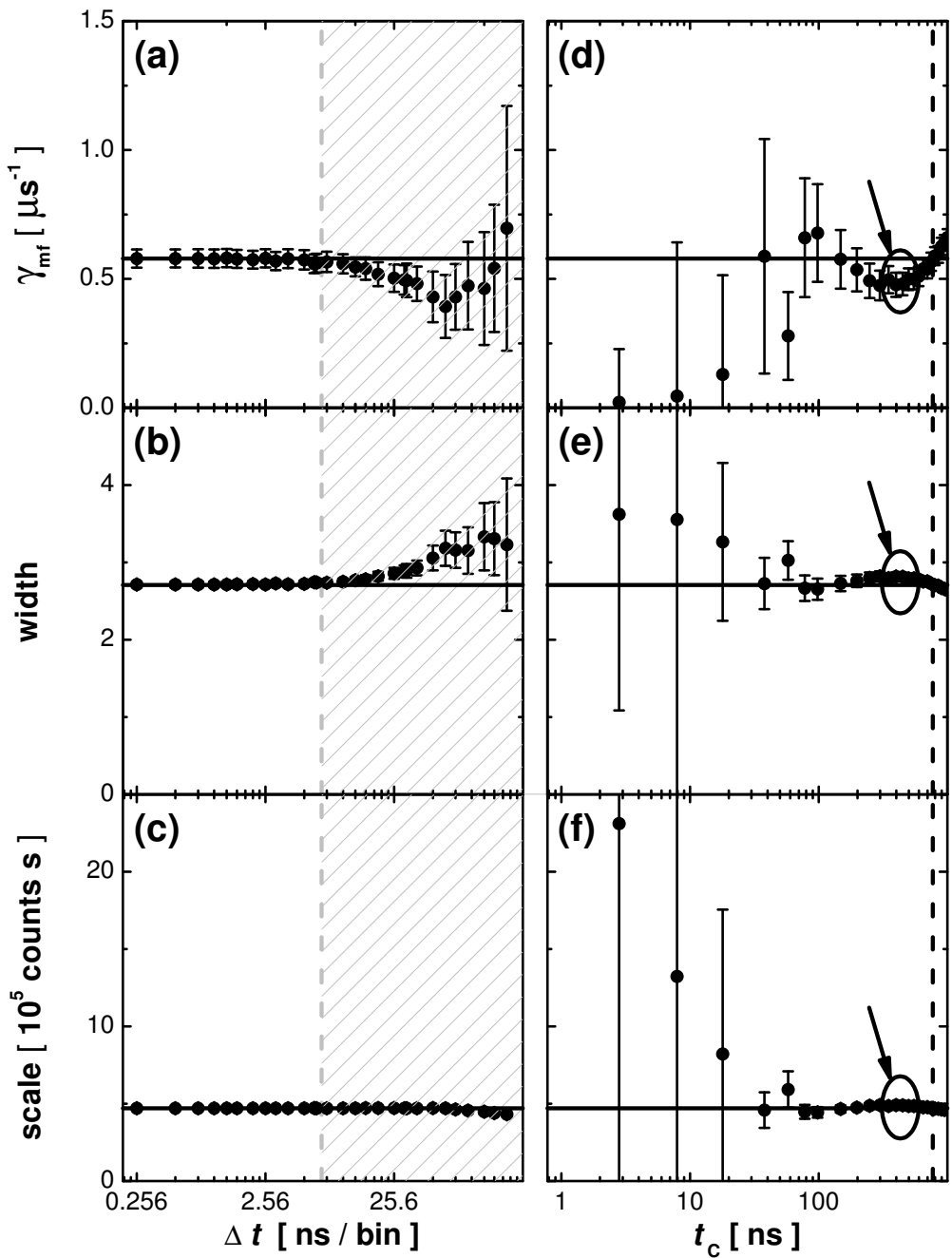

Figure 5.8: Results for the $\gamma_{\mathrm{mf}}$, width and scale parameters used in a log-normal decay rate distribution model. (a to c) Dependence on time bin width $\Delta t$, for a time range $t \in[2.048,767.744] \mathrm{ns}$. The hatched area for $\Delta t>7 \mathrm{~ns}$ indicates the region where the parameter values become unreliable. ( $d$ to $f$ ) Dependence on the time cut-off $t_{\mathrm{C}}$, for a fixed time bin width $\Delta t=0.256 \mathrm{~ns}$. The horizontal line is a guide to the eye from results in figures a to $c$. The vertical dashed line represents $t_{\mathrm{C}}$ used in figures a to $c$. Circles mark the time where the emission signal becomes smaller than the standard deviation in the background. If $t_{\mathrm{C}}$ is larger than this encircled region the parameters start to deviate. For small values of $t_{\mathrm{C}}$ the results show very large uncertainties. The model is used to describe a single decay curve measured from $\mathrm{PbSe}$ quantum dots in a titania inverse opal, at wavelength $\lambda=1300 \mathrm{~nm}$. The first 2.048 ns behind the decay curve maximum are disregarded and error bars represent the standard deviation calculated by TableCurve2D. 
ticipated to describe the PbSe quantum dot decay from $3 \mathrm{D}$ photonic crystals. As the dots are not located at the same position they experience different densities of optical states [33]. Consequently, each quantum dot has a different spontaneous emission decay rate. Hence, for a quantum dot ensemble, the measured decay curve results from a sum of different decay rate contributions, i.e.,

$$
I(t)=\Sigma_{i} \alpha_{i} \exp \left(\gamma_{i} t\right)
$$

where $\alpha_{i}$ is the weighting factor for decay rate $\gamma_{i}$, and $\Sigma \alpha_{i}$ is normalized to unity.

The open question remains which model is the best candidate to describe the data. The following requirements are posed to decide which model is most appropriate:

1. Preferably, the model should follow from first principles.

2. The goodness of fit $\chi_{\text {red }}^{2}$ should be close to one.

3. The number of free parameters should be as small as possible.

4. The model should not allow the use of un-physical parameter ranges (e.g., no negative decay rates).

Below, four different models are considered: 1) single-exponential decay, 2) biexponential decay, 3-4) log-normal distribution of decay rates. The Kohlrausch stretched-exponential model [227] is not included as its normalization is ill defined for emitters with a realistic quantum efficiency of less than $100 \%$ [226].

Single-exponential intensity decay $I(t)$ is defined as

$$
I(t)=I_{0} e^{-\gamma t}
$$

where $t$ is the running time-parameter. The model contains only two fit parameters: the scaling factor $I_{0}$ and the decay rate $\gamma$. The bi-exponential model is given by

$$
I(t)=I_{1} e^{-\gamma_{1} t}+I_{2} e^{-\gamma_{2} t}
$$

and already contains four fit parameters, i.e., contributions from two decay rates $\left(\gamma_{1}\right.$ and $\left.\gamma_{2}\right)$, each with their own scaling factor ( $I_{1}$ and $I_{2}$ respectively).

So far, isolated decay rates were used, each with their own weighting factor. It is also possible to change the summation in Equation 5.14 into an integral and use a distribution function $\phi(\gamma)$ to describe the weighting. This yields

$$
I(t)=I_{0} \int_{0}^{\infty} \mathrm{d} \gamma \phi(\gamma) e^{-\gamma t} .
$$

$\gamma$ being the decay rate, $t$ the time, and $I_{0}$ a constant to scale the calculated distribution to the experimentally obtained decay curve. A log-normal distribution of decay rates $\phi_{\mathrm{LN}}(\gamma)$ was successfully employed to fit decay curves measured from CdSe quantum dots inside titania inverse opals [33]. Here, the distribution $\phi_{\mathrm{LN}}(\gamma)$ 
is defined as

$$
\phi_{\mathrm{LN}}(\gamma)=A \exp \left(-\frac{\ln ^{2}\left(\gamma / \gamma_{\mathrm{mf}}\right)}{w^{2}}\right),
$$

where $\gamma_{\mathrm{mf}}$ is the most frequent decay rate, and $w$ is the width parameter which is related to the width of the distribution at its $1 / e$ height $\Delta \gamma$ via:

$$
\Delta \gamma=2 \gamma_{\mathrm{mf}} \sinh (w) .
$$

To normalize the distribution, factor $A$ is equal to

$$
A=\left[\gamma_{\mathrm{mf}} w \sqrt{\pi} \exp \left(w^{2} / 4\right)\right]^{-1} .
$$

To speed up the fit routine the three fit parameters $\left(I_{0}, \gamma_{\mathrm{mf}}\right.$, and $\left.w\right)$ were rewritten to scale $=I_{0} A, \gamma_{\mathrm{mf}}$, and width $=w$.

The true log-normal distribution ${ }^{\text {iv }} \phi_{\mathrm{TLN}}(\gamma)$ is only slightly different from Equation 5.18 and therefore added to the list of possible models to fit the data. $\phi_{\mathrm{TLN}}(\gamma)$ is defined as

$$
\phi_{\mathrm{TLN}}(\gamma)=\frac{1}{\gamma \sigma \sqrt{2 \pi}} \exp \left(-\frac{(\ln (\gamma)-\mu)^{2}}{2 \sigma^{2}}\right),
$$

with $\mu$ and $\sigma$ the mean and standard deviation of the distribution. This model has three fit parameters $\left(I_{0}, \mu\right.$, and $\left.\sigma\right)$.

TableCurve2D v5.0 software is used to fit all four models to the same decay curve data. Figure 5.9 shows the results. Both the single- and bi-exponential model are discarded as the residuals are not centered around zero, and because of the high $\chi_{\text {red }}^{2}$. Both log-normal distribution models look similar at first sight. However, a close look at the residuals shows that distribution $\phi_{\mathrm{LN}}(\gamma)$ yields a better fit, especially at short times. This is in accordance with $\chi_{\text {red }}^{2}$ being closer to one for this model. The log-normal model that uses $\phi_{\mathrm{LN}}(\gamma)$ (Equation 5.18) is preferred over the true log-normal model for further analysis of decay curve measurements. The $\phi_{\mathrm{LN}(\gamma)}$ model was also successfully applied in earlier studies on spontaneous emission from light sources inside photonic crystals [33, 226].

Figure 5.10 contains the results of the single-exponential model and the lognormal model fitted to the same decay curve from PbSe quantum dots emission in a hexane suspension. The $\chi_{\text {red }}^{2}=1.00$ clearly shows the single-exponential behavior of these dots in suspension. The log-normal distribution yields $\chi_{\text {red }}^{2}=0.99$. The curvature observed in the photonic crystals (Figure 5.9) is not observed here as the quantum dots all experience the same local density of optical states. The results from Figures 5.9 and 5.10 show the robustness of this log normal distribution model, that uses only three parameters to fit a large range of decay curve shapes.

${ }^{\text {iv }}$ i.e., the distribution of a random variable $(\gamma)$ whose logarithm is normally distributed. 

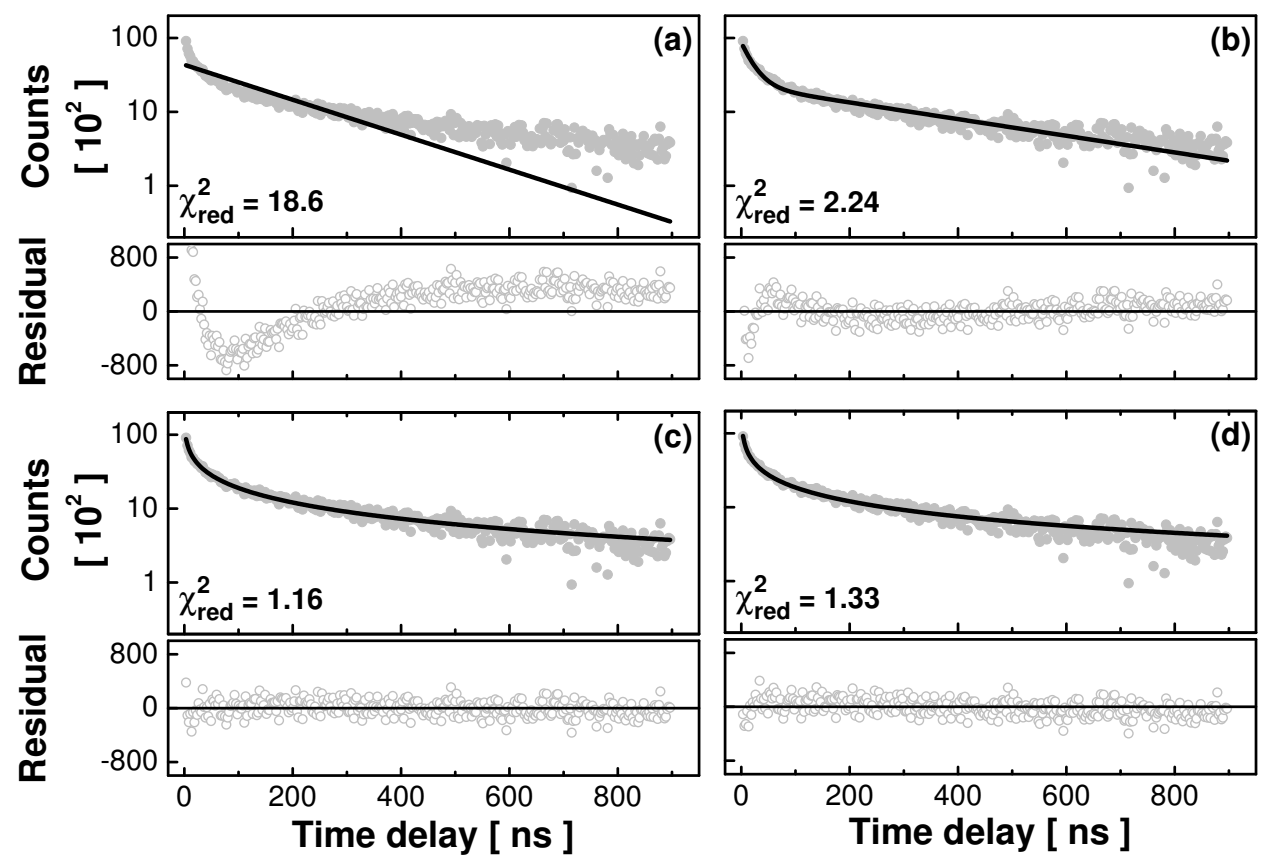

Figure 5.9: Comparison of four different models used to fit an emission decay curve of PbSe quantum dots in a titania inverse opal (lattice parameter $a=349 \mathrm{~nm}$ ), measured at $\lambda=1300 \mathrm{~nm}$. (a) single exponential, (b) bi-exponential, (c) a lognormal distribution of decay rates $\phi_{\mathrm{LN}}(\gamma)$, and (d) the true log-normal distribution of decay rates $\phi_{\mathrm{TLN}}(\gamma)$. Gray dots represent the measured decay curve, and black curves are the result of the fit. Open circles are the corresponding residuals. The goodness of fit $\chi_{\text {red }}^{2}$ is shown for each model.

\subsubsection{Outcome decay curve model: a parameter study}

Before the data from different experiments can be compared the outcome of the model is evaluated for a single data set. Therefore, TableCurve2D is used to combine the log-normal distribution model with the decay curve data from Figure 5.6.(a) $(a=1351 \mathrm{~nm})$. The fit routine is very robust, as the results for $\gamma_{\mathrm{mf}}$, scale, and width do not depend on the starting conditions.

We want to verify if there are more solutions for the model parameters that describe the data. Therefore, the $\chi_{\text {red }}^{2}$ was calculated for parameter ranges that overlap the result from the model, using Matlab v7.4.0.287 (R2007a). Figure 5.11 shows the calculated $\chi_{\text {red }}^{2}$ landscape. It is observed that a large, elongated volume exists for which the parameters yield $\chi_{\text {red }}^{2} \approx 1$. Therefore, many parameter combinations are suitable to describe the decay curve. Outside this volume the $\chi_{\text {red }}^{2}$ rapidly increases to values much larger than one. As there was no other region 

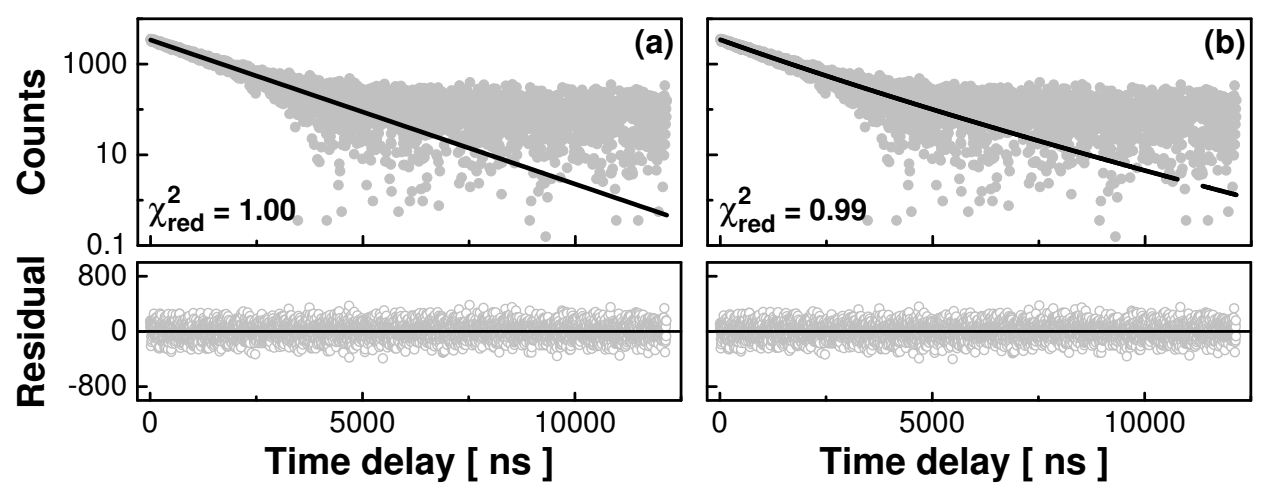

Figure 5.10: Comparison of two models used to fit a decay curve of PbSe quantum dots in a hexane suspension, measured at $\lambda=1300 \mathrm{~nm}$. (a) Single-exponential model. (b) Log-normal distribution of decay rates model. Gray dots represent the measured decay curve, and black curves are the result of the fit. Open circles are the corresponding residuals. The goodness of fit $\chi_{\text {red }}^{2}$ is shown for each model. Clearly, quantum dots suspended in hexane show single-exponential emission decay. Furthermore, the figure demonstrates that the log-normal distribution can also describe single-exponential decay.

observed with $\chi_{\text {red }}^{2} \approx 1$, Figure 5.11 shows the global optimum for the model. Furthermore, the figure shows that even when the value for $\gamma_{\mathrm{mf}}$ is increased by $60 \%$ the model can describe the data well.

To understand why different decay rate distributions yield the same decay curve two distributions are plotted. Both distributions stem from parameter sets that lie within the iso- $\chi_{\text {red }}^{2}$-surface of Figure 5.11. Figure 5.12 shows both distributions. The distributions overlap over a large range of decay rates, in analogy to the range prescribed by the Sampling Theorem ${ }^{\mathrm{v}}$ [228]. As $\gamma_{\mathrm{mf}}$ lies outside this range its value is uncertain. Hence, direct comparison of the parameters found for different decay curves is questionable. As discussed in the following section, the unhatched area from Figure 5.12 can be used to compare the measured decay curves.

\subsubsection{Average and variance of decay-rate distribution}

As shown in the previous section, the model used to describe the measured decay curves yields broad decay rate distributions. The experiment only resolves part of the decay rate range depicted by the distribution. In this region, set by $\gamma \in$ $\left[\gamma_{\min }, \gamma_{\max }\right]$, distributions that properly describe the same decay curve overlap. To

\footnotetext{
${ }^{\mathrm{v}}$ For a total measurement time of $900 \mathrm{~ns}$, and a sampling rate $f_{\mathrm{s}}=1 / \Delta t=0.39 \mathrm{GHz}$ one can reconstruct frequency components in the range from of $2 \cdot 1 / 900$ to $2 \cdot 0.39 \mathrm{GHz}$, according to this sampling theorem.
} 


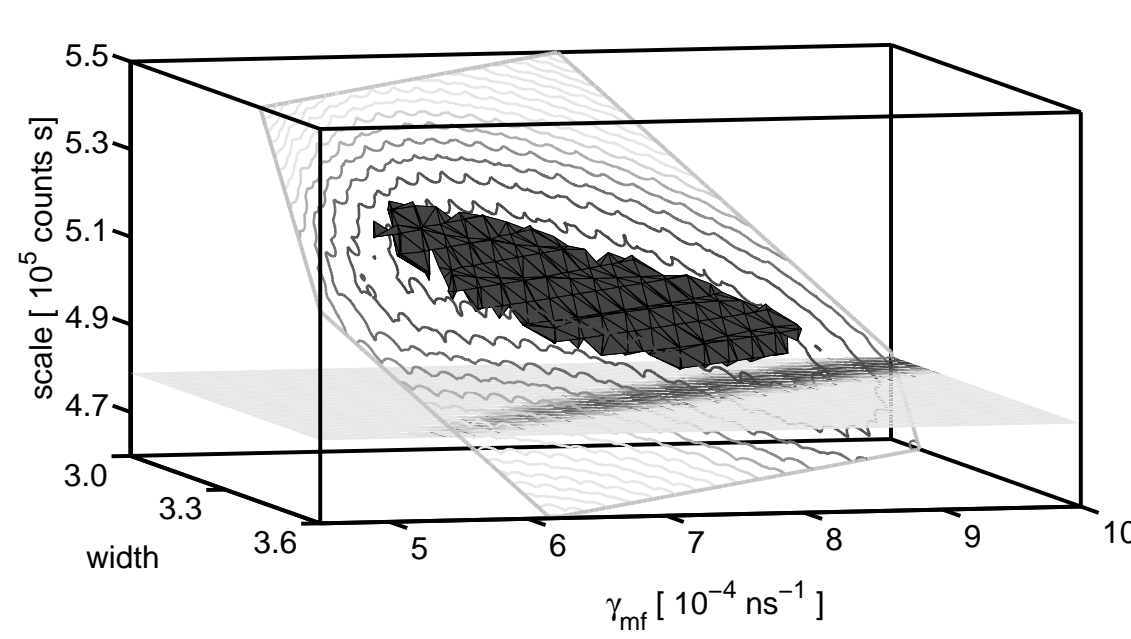

Figure 5.11: Goodness of fit $\chi_{\text {red }}^{2}$ versus model parameters. The central, dark surface shows the calculated $\chi_{\text {red }}^{2}=1.26$ iso-surface landscape. A plane of contour plots clarifies the placement and orientation of the iso- $\chi_{r e d}^{2}$-surface. The solid slice plane at scale $\approx 4.8 \cdot 10^{5}$ counts.s shows the shape of the iso- $\chi_{\text {red }}^{2}$-surface in its flattened direction. There exists a large volume in parameter space for which the model describes the data well. Outside this volume the goodness of fit deteriorates rapidly. Additional volumes with $\chi_{\text {red }}^{2} \approx 1$ were not observed.

compare different decay curves the mean decay rates and variances are calculated, using only information in the region limited by $\gamma_{\min }$ and $\gamma_{\max }$.

For this limited region, the mean decay rate $\langle\gamma\rangle_{\text {lim }}$ is given by [229]:

$$
\langle\gamma\rangle_{\lim }=\frac{1}{N_{\lim }} \int_{\gamma_{\min }}^{\gamma_{\max }} \mathrm{d} \gamma \gamma \phi_{\mathrm{LN}}(\gamma)
$$

Here, $\phi_{\mathrm{LN}}(\gamma)$ is the distribution of decay rates - that is chosen to be log-normal and the normalization factor $N_{\text {lim }}$ is defined as

$$
N_{\lim }=\int_{\gamma_{\min }}^{\gamma_{\max }} \mathrm{d} \gamma \phi_{\mathrm{LN}}(\gamma)
$$

If the $\gamma$-range is not limited (i.e., $\gamma \in[0, \infty\rangle$ ) the normalization is 1 and Equation 5.22 simply yields the distributions' average decay rate $\langle\gamma\rangle$ of

$$
\langle\gamma\rangle=\gamma_{\mathrm{mf}} \exp \left(\frac{3}{4} w^{2}\right)
$$

The variance $\sigma^{2}$, or second moment, of the distribution calculated over a limited 


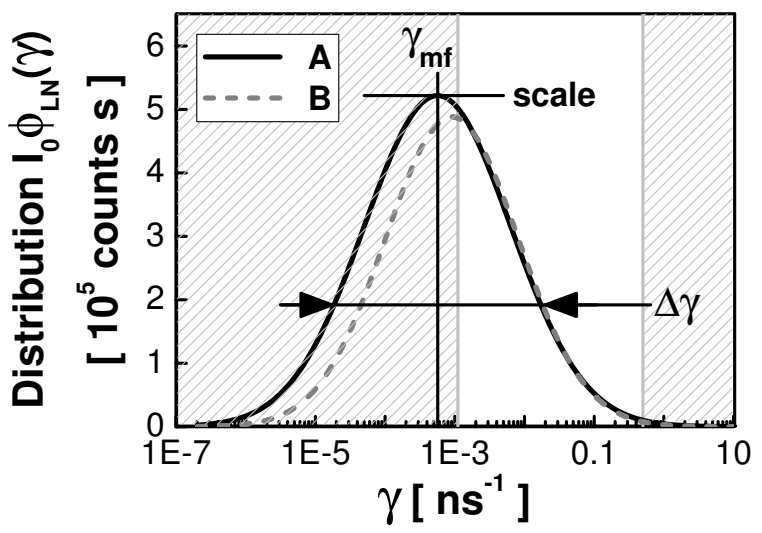

Figure 5.12: Two distributions from parameter sets that accurately describe the same decay curve. The parameters used stem from the results in Figure 5.11: A) $\gamma_{\mathrm{mf}}=5.646 \mathrm{E}-4 \mathrm{~ns}^{-1}$, width $=3.417$, scale $=5.219 \mathrm{E} 5$ counts $\mathrm{s}$, and B) $\gamma_{\mathrm{mf}}=$ $9.083 \mathrm{E}-4 \mathrm{~ns}^{-1}$, width $=3.107$, scale $=4.884 \cdot 10^{5}$ counts $\cdot \mathrm{s} . \gamma_{\mathrm{mf}}$ and $\Delta \gamma$ define the position and the $e^{-1}$ width of distribution respectively. The unhatched area shows the $\gamma$-range that can be recovered from the experiment according to the sampling theorem. In this range both distributions practically overlap.

decay rate range is [229]

$$
\sigma_{\lim }^{2}=\frac{1}{N_{\lim }} \int_{\gamma_{\min }}^{\gamma_{\max }} \mathrm{d} \gamma \gamma^{2} \phi_{\mathrm{LN}}(\gamma)
$$

The average decay rates and variances were calculated for eight distributions that all describe the same decay curve properly. The distributions stem from different parameter sets that are inside the volume bound by the iso- $\chi_{\text {red }}^{2}$-surface from Figure 5.11. Figure 5.13 shows the results for $\langle\gamma\rangle_{\lim }$ and $\sigma_{\lim }^{2}$. The individual parameters that determine the different distributions change up to $60 \%$ (see Table D.1 on page 138 for details). However, all distributions yield practically the same $\langle\gamma\rangle_{\lim }$ and $\sigma_{\lim }^{2}$. The average values of the results are also plotted with an error bar that is the standard deviation. The standard deviation is only $3 \%$ of the mean value, both for $\langle\gamma\rangle_{\lim }$ and $\sigma_{\lim }^{2}$. This small standard deviation make the average decay rate $\langle\gamma\rangle_{\lim }$ and the variance $\sigma_{\lim }^{2}$ very efficient and meaningful measures to compare decay curves.

\subsection{Conclusions}

This chapter addressed the challenges encountered in near-infrared time-resolved emission experiments. Clearly, time correlated single photon counting is much more 


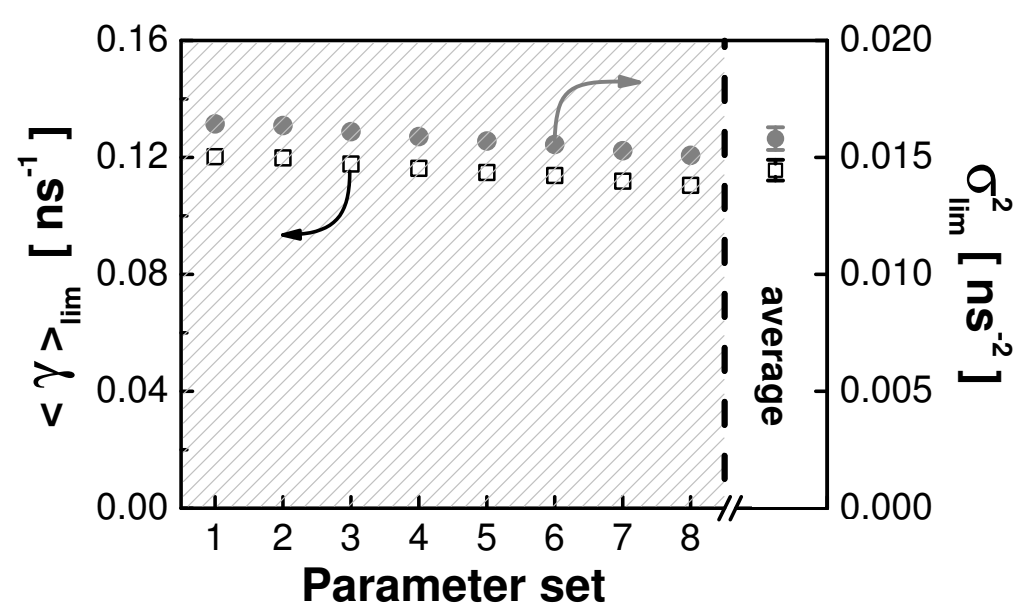

Figure 5.13: Calculated mean decay rates $\langle\gamma\rangle_{\lim }$ (open squares) and variances $\sigma_{\lim }^{2}$ (closed dots) for parameter sets in the $\chi_{\text {red }}^{2} \approx 1$ volume of Figure 5.11. The decay rate range used was limited by $\gamma_{\min }=1 / 900 \mathrm{GHz}$ and $\gamma_{\max }=1 / 2 \mathrm{GHz}$ (open area in Figure 5.12. Hatched area contains the results of eight calculations. The arrows point towards the relevant ordinate. The open area shows the average outcome of the calculations. The error bar is the derived standard deviation and equals only $3 \%$ of the mean value.

difficult in this wavelength range as compared to the visible range. The discrepancy originates from the difference in dark signal of the photon-detectors used. Photomultiplier tubes display typically 2000 times more dark signal and a 20 times lower quantum efficiency in the near-infrared, as compared to the visible. Furthermore, the signal to noise ratio in the visible regime is intrinsically better as the efficacious repetition rates are higher. The high background signal gives rise to an additional pitfall when the background subtracted decay curve data are modeled. For calculations of the goodness of fit $\chi_{\text {red }}^{2}$ the normal assumption that the modeled data shows Poisson noise, does not hold. Instead, the variance in the raw data must be used to calculate $\chi_{\text {red }}^{2}$.

The light sources of interest are PbSe quantum dots. A good model to describe the emission decay from these quantum dots inside titania inverse opals is based on a log-normal distributions of decay rates. However, the parameters that define the distributions are outside the measurement range, and correlated. Hence, using these parameters to compare different decay curves gives rise to large uncertainties.

We demonstrated that log-normal distributions of decay rates, that describe the same decay curve, overlap in the region probed by the measurement. Subsequently, the average decay rates and variances of the distributions were calculated in this region. These rates are practically the same for each distribution. This also holds for the variances. Both rate and variance exhibit a standard deviation of only 
$3 \%$ of their mean values. Hence, we are able to measure time-resolved emission intensity decay at telecom wavelengths. Furthermore, with the average decay rate and variance, we derived the very quantitative measures needed to describe and compare decay curves in near-infrared emission experiments. 


\section{Spontaneous emission rates of near-infrared quantum dots controlled with photonic crystals}

\subsection{Introduction}

Radiative properties of excited emitters are determined both by characteristics of the emitter and by its environment [6, 230-233]. In general, the radiative decay rate is described by Fermi's Golden Rule, see Section 1.1 and References [12, 13]. From this Golden Rule it was recognized that the spontaneous emission rate is proportional to a local property called the Local Density of Optical States (LDOS) [16]. It is possible to control the LDOS using dielectric nanostructures. As a result, the spontaneous emission rate of light sources embedded in such nanostructures can be enhanced or inhibited [16, 234-236].

To change the spontaneous emission rate of a light source by means of the LDOS, the source can be placed in many different systems. A straightforward example is an emitter near an interface, where the LDOS depends on the distance between emitter and interface $[162,174,230,237]$. As a simplification we propose to classify the systems based on the number of resonances to which the emitter effectively couples.

(i) In cavities, Fabry-Pérot resonators, and near field antennas a light source can be coupled effectively to a single resonance when the frequency of the source matches the resonance frequency of the structure. This resonance mode gives rise to a peak in the LDOS ${ }^{\mathrm{i}}$, thereby enhancing the spontaneous emission rate of the

\footnotetext{
${ }^{\mathrm{i}}$ In practice this emitter-resonance system is coupled to a continuum of bath states which broadens the resonance peak in the $\operatorname{LDOS}[7,74]$
} 
coupled emitters [20-27]. Control of spontaneous emission decay by means of such resonator systems implies narrow bandwidth and small volumes, as the emitter frequency must match the resonance mode, and the emitter needs to be located close to the cavity.

(ii) In contrast, a source inside a photonic crystal is coupled to an infinite number of Bloch modes [55]. These modes all contribute to the LDOS, which shows broad crests and troughs as a function of frequency. Therefore, photonic crystals enable broadband control of spontaneous emission rates of large volumes of emitters [30, 33-36]. Furthermore, the extreme case of total inhibition of spontaneous emission is predicted for light sources inside photonic crystals that exhibit a band gap [1]. Only photonic crystals exhibit such a band gap. In the frequency range of this gap the LDOS is zero, which means that all electromagnetic field modes are expelled from the structure. Consequently, an excited emitter whose transition frequency lies inside the band gap remains excited as there are no field modes to which it can couple. Here we study emission in photonic crystals.

To create a crystal with a photonic band gap, a large dielectric contrast is needed. From Moss' law it is known that materials with a high dielectric constant have correspondingly a low electronic band gap [238]. Consequently, the onset of absorption lies at longer wavelengths for materials with a higher dielectric constant. Silicon has a high refractive index $(n=3.5)$ and is therefore a good candidate for the fabrication of photonic crystals with a band gap [184, 239]. In addition, silicon is a very popular material used in many applications. Because of silicon's relatively small electronic band gap $(1.12 \mathrm{eV})$, silicon is only transparent at wavelengths longer than $1100 \mathrm{~nm}$. Therefore, the aim is to control spontaneous emission rates at wavelengths $\lambda>1100 \mathrm{~nm}$. These near-infrared wavelengths also happen to overlap the telecom-range, which make our results potentially interesting for a large industry.

Using 2D slab geometry photonic crystals, the first control of spontaneous emission decay at near-infrared wavelengths $\lambda>1100 \mathrm{~nm}$ was demonstrated with quantum wells at temperatures $T=4 \mathrm{~K}$ [31]. Using emitters inside 3D photonic crystals In the same wavelength range, enhancement and inhibition of spontaneous emission decay was claimed at room temperature [83, 218, 240, 241]. However, these last results leave room for questions as we will discuss in Section 6.3.4.

In this chapter, we demonstrate the first control over spontaneous emission decay in 3D photonic crystals at near-infrared wavelengths $\lambda>1100 \mathrm{~nm}$. To this end PbSe quantum dots are studied inside titania inverse opals. In this way, a relatively new type of light source is combined with a well-known photonic crystal. Section 6.2 contains the experimental details, followed by results and discussions in Section 6.3. Conclusions are in Section 6.4.

\subsection{Experimental details}

A demonstration of spontaneous emission decay control requires a comparison between decay rates [15]. Using Fermi's Golden Rule the spontaneous emission rate 
of a dipole emitter can be calculated inside a photonic crystal or in vacuum. If all parameters in this equation would be known, it is possible to calculate the LDOS and the decay rate and compare these to a single absolute decay curve measurement to show the influence of the photonic crystal on the spontaneous emission rate of the light source. However, in practice the emitters' position, orientation, and transition dipole-moment are not known. Consequently, relative measurements on isolated emitters are needed to demonstrate control of spontaneous emission decay with photonic crystals.

To modify an emitters' decay rate in different measurements, only one experimental parameter should be changed. For photonic crystals, parameters that are related to the relevant LDOS are the photonic crystals' lattice parameter $a$ and the emission frequency $\omega=2 \pi c / \lambda$. Therefore, a typical parameter in this study is the ratio $a / \lambda$, which is known as the reduced frequency. As shown in Chapter 3, changing the emission frequency must be done with great care, as a change in wavelength corresponds to looking at different sizes of quantum dots that may have different transition dipole moments, or experience different non-radiative decay rates. Hence, it is preferred to compare results measured at the same wavelength, and control the LDOS via the lattice parameter.

Thus, to demonstrate control of spontaneous emission with photonic crystals we need to fabricate crystals with different lattice parameters. To this end, titania inverse opals were used. The lattice parameters are chosen such that light sources inside the crystal probe the frequency range of the crystals' pseudogap, where the DOS is depleted [15]. This pseudogap is in the frequency range starting at and just above the L-gap, the stop gap associated with the lowest order Bragg diffraction. This is exactly the frequency range of interest in Chapter 4 . Therefore, the same range of crystal lattice parameters is needed here: $a \in[670 ; 1230] \mathrm{nm}$. The error in the values for $a / \lambda$ are determined by the standard deviation of the stopband frequency, as derived from twenty reflectivity measurements on nine different samples.

After fabrication of the crystals the isolated light sources should be added. Therefore, the structures were infiltrated with suspensions that contain low concentrations of PbSe quantum dots to prevent absorption processes that influence the decay rate [151]. Additional details on the fabrication and infiltration of these samples, and the preparation for experiments can be found in Sections 4.2.1 and 4.2.2.

Time-resolved emission experiments are needed to determine the spontaneous emission rates of the quantum dots in the samples with different lattice parameters. Therefore, a home-built experimental setup was established, as explained in Chapter 2. This setup allows for spatially, and spectrally resolved time-correlated single photon counting. Technical aspects of time-correlated single photon counting at near-infrared wavelengths were discussed in detail in Chapter 5. Below the experimental procedure for our time-resolved emission experiments are summarized. The elements of this procedure were addressed in Chapters 4 and 5 .

First, reflectivity measurements were done on the photonic crystals to find the 
regions that show clear stop bands. These regions were used in the emission experiments. Secondly, the crystal was infiltrated with PbSe quantum dots suspended in hexane, dried, rinsed with hexane, glued to a needle pin, and placed in a sealed chamber under a nitrogen atmosphere. For each sample the decay curves were measured by the same procedure to minimize systematic effects from possible temporal changes of the quantum dots. The dots were excited with a pulsed laser that operates at a wavelength $\lambda=532 \mathrm{~nm}$, and at a repetition rate of $204 \mathrm{kHz}$. The excitation power of typically a few microWatts was set at the beginning of the experiment and chosen such that the detected emission rate is smaller than $1 \%$ of the excitation rate. Decay curves were measured at three different wavelengths, in chronological order: $\lambda \in\{1300,1250,1450\} \mathrm{nm}$. At each wavelength the decay curve was measured in five subsequent time slots of $10 \mathrm{~min}$. The results of these measurements were added. Emission spectra were taken before and after measuring a set of five decay curves at the same wavelength. After all these measurements an additional decay curve was measured at $\lambda=1300 \mathrm{~nm}$ to judge changes of the results in time. The data were averaged in time to obtain time bins of $2.56 \mathrm{~ns}$, and the average background value was subtracted. Finally, the decay curve data measured between times $2<t<900$ ns were described by a model based on a lognormal distribution of decay rates. The obtained decay rate distributions was used to compare different decay curves. As quantitative measures for this comparison the distributions' average decay rate $\langle\gamma\rangle_{\lim }$ and variance $\sigma_{\lim }^{2}$ were derived over a limited decay rate range from $\gamma_{\min }=1 / 900 \mathrm{GHz}$ to $\gamma_{\max }=1 / 2 \mathrm{GHz}$. The error in $\langle\gamma\rangle_{\lim }$ and $\sigma_{\lim }^{2}$ was determined to be $3 \%$ of their values.

Figure 6.1 shows a typical example of a decay curve measured from PbSe quantum dots inside a titania inverse opal with lattice parameter $a=369 \mathrm{~nm}$. The top window contains the histogram of the time delays between the excitation of the sample and the detection of single photon photoluminescence events (gray dots). The data are corrected for background signal and averaged over subsequent timebins. For each time delay bin the averaged number of events (counts) is plotted on the vertical axis. The solid line shows the result of the model that describes the measured decay curve, and $\chi_{\text {red }}^{2}$ represents the goodness of fit between the model and the data (see Section 5.3.2). The difference between the measured data and the model, i.e., the residuals, are plotted in the bottom window. As the residuals are evenly distributed around zero for all time delays, the model describes the data well.

\subsection{Results and discussion}

\subsubsection{Decay curves measurements}

Figure 6.2.(a) shows decay curves measured at a fixed wavelength $\lambda=1300 \mathrm{~nm}$ from two samples with different lattice parameters $a$. Non-exponential decay is observed, which is due to the ensemble averaging of the emission from many isolated quantum 


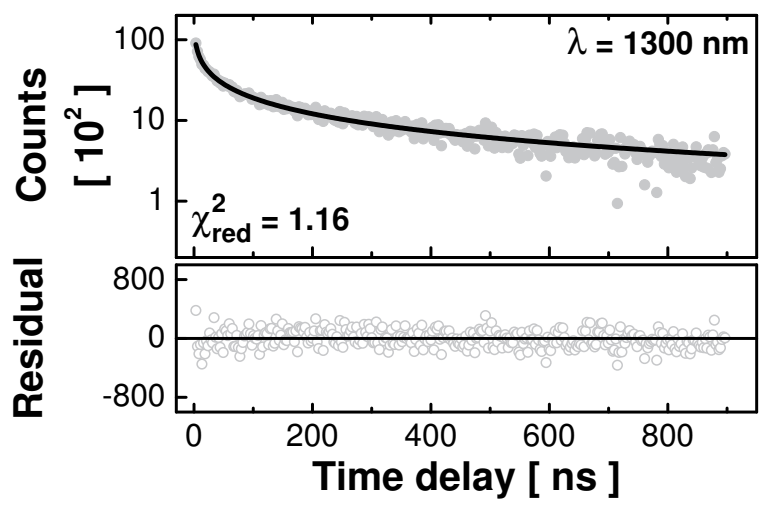

Figure 6.1: Example of PbSe quantum dot decay from a titania inverse opal, measured a wavelength $\lambda=1300 \mathrm{~nm}$. Gray dots are measured data, and open circles show the residual counts when the data are compared to a model (solid curve). The model uses a log-normal distribution of decay rates to describe the intensity decay curve. The average background was subtracted from the data and the data were averaged over five time bins.

dots that all experience a different LDOS, and consequently emit with different rates. The decay curve measured at reduced frequency $a / \lambda=0.57$ is less curved than the decay curve measured at $a / \lambda=0.87$. Therefore, the former spontaneous emission rate appears smaller than the rate of the latter. The difference in radiative decay is explained by a change in the LDOS, as shown in Section 6.3.5. Clearly these curves demonstrate the ability to control the spontaneous emission rate of light sources at near-infrared wavelengths, using photonic crystals.

Figure 6.2.(b) shows three decay curves measured from a single sample (fixed a) at different emission wavelengths $\lambda$. These emission wavelengths are below any gap-regions in the LDOS. Clearly, an increase in emission wavelength gives rise to a faster spontaneous emission rate. As discussed in Section 3.5 this decay rate dependence on wavelength is attributed to the oscillator strength which increases with $\mathrm{PbSe}$ quantum dot size and dominates the changes in the LDOS. Therefore, a claim about spontaneous emission decay rates controlled with photonic crystals (read: LDOS) should be inferred from measurements at the same wavelength, as in Figure 6.2.(a).

\subsubsection{Temporal change of measured decay curves}

As shown in Section 4.3.1, the quantum dot emission spectrum may change in time under illumination. Therefore, a very strict protocol was adopted to allow for the comparison of data sets measured at the same wavelength. This protocol prescribed that decay curve measurements should start and end at emission wavelength $\lambda=$ 

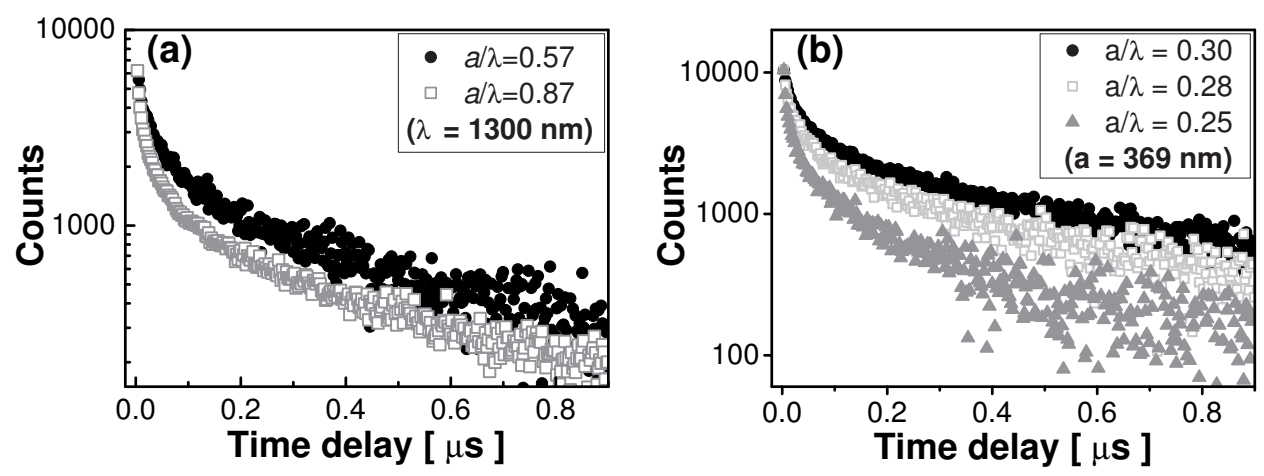

Figure 6.2: Decay curves of PbSe quantum dots inside titania inverse opals. (a) Decay curves measured at $\lambda=1300 \mathrm{~nm}$ in samples with different lattice parameters: $a=740 \mathrm{~nm}$ (black dots), and $a=1130 \mathrm{~nm}$ (open squares). The quantum dots in the sample with the largest $a$ decay fastest. The $a / \lambda=0.87$ data were multiplied by 0.345 to overlap the $a / \lambda=0.57$ data at zero time delay. (b) Decay curves measured from the same position in a titania inverse opal $(a=369 \mathrm{~nm})$ at three different wavelengths $\lambda$ : $1250 \mathrm{~nm}$ (black dots), $1300 \mathrm{~nm}$ (open squares), and $1450 \mathrm{~nm}$ (closed triangles). With increasing wavelength the decay curves show a faster decrease with time. Hence, the decay rate increases with wavelength. The average background signal was subtracted from each curve and the data were averaged to obtain time bins of 2.56 ns. The $a / \lambda=0.28$ and $a / \lambda=0.25$ data were multiplied by 1.14 and 0.947 , respectively, to overlap the $a / \lambda=0.30$ data at zero time delay.

$1300 \mathrm{~nm}$, to enable the evaluation of temporal changes in the decay curve. Figure 6.3 shows a typical result of two decay curves measured at $\lambda=1300 \mathrm{~nm}$. The second measurement shows slightly slower decay. The average decay rates found for these curves are $\langle\gamma\rangle_{\lim }=0.10 \mathrm{~ns}^{-1}$ for the first and $\langle\gamma\rangle_{\lim }=0.092 \mathrm{~ns}^{-1}$ for the second measurement, respectively. Hence, the average decay rate decreased by $12 \%$ during the time of the experiment. In the same way, the temporal change of the decay rate was studied ten times. We measured seven different locations from six samples. Six measurements revealed a reduction and four measurements showed an increase of the average decay rate with time. From these measurements, the mean change of the average emission rate is $-2 \%$, with a standard deviation of $13 \%$. Therefore, the $13 \%$ standard deviation can be used as a very conservative upper bound for the uncertainty in the measured average emission rates. Since the measured variations of the average emission rates measured at different reduced frequencies $a / \lambda$ will appear to be much larger than $13 \%$, we can therefore conclude that such variations are a significant physical effect. 


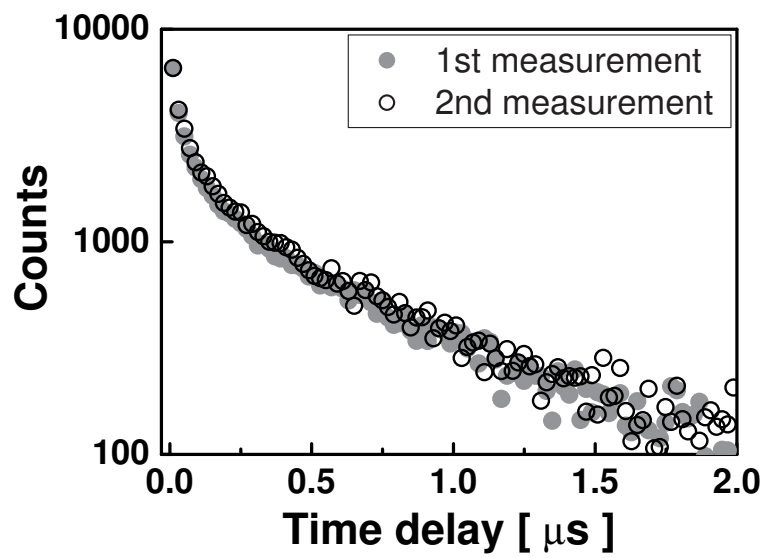

Figure 6.3: Background subtracted and decay curves measured from PbSe quantum dots inside a titania inverse opal $(\mathrm{a}=933 \mathrm{~nm})$. Both curves are measured from the same location on the sample. The second measurement data (open circles) are multiplied by 1.15 to overlap the first measurement at the shortest delay time. Especially at time delays below $1 \mu \mathrm{s}$ the second decay curve is above the first curve. Hence, the second measurement shows slower decay. The second measurement started 2.7 hours after the first. For clarity, the variance in the data is reduced by averaging over 39 time bins of 512 ps.

\subsubsection{Inhibition and enhancement of spontaneous emission rate versus lattice parameter and wavelength}

In this section, the experimental results will be discussed quantitatively by comparing the average decay rates $\langle\gamma\rangle_{\lim }$ and variances $\sigma_{\lim }^{2}$ of the decay rate distributions that describe the intensity decay curve. Figure 6.4 shows the average decay rate $\langle\gamma\rangle_{\text {lim }}$ and variance $\sigma_{\text {lim }}^{2}$ from the measurements on PbSe quantum dots inside titania inverse opals as a function of reduced frequency $(a / \lambda)$. For clarity the data are separated based on their emission wavelength. The symbols denote the measured data, interconnected with a dashed line that serves as a guide to the eye. First the average decay rates are discussed, subsequently, the variances of the decay rates.

Figures 6.4.(a-c) show how the average decay rate changes as a function of reduced frequency. In the long wavelength limit, $a / \lambda<0.45,\langle\gamma\rangle_{\lim }$ is practically constant at a value that is marked by the horizontal dashed line. Compared to this long wavelength limit, all measured wavelengths reveal an inhibition of the average decay rate around $a / \lambda=0.6$, and an enhancement around $a / \lambda=0.85$. The vertical gray bar at $a / \lambda=0.77$ corresponds to the pseudogap region calculated in Reference [242] and discussed in Section 6.3.5. In Figure 6.4.(b) arrows point out the ranges where the decay rates are enhanced or inhibited with respect to the long-wavelength limit. The measured maximum, minimum, and long wavelength 


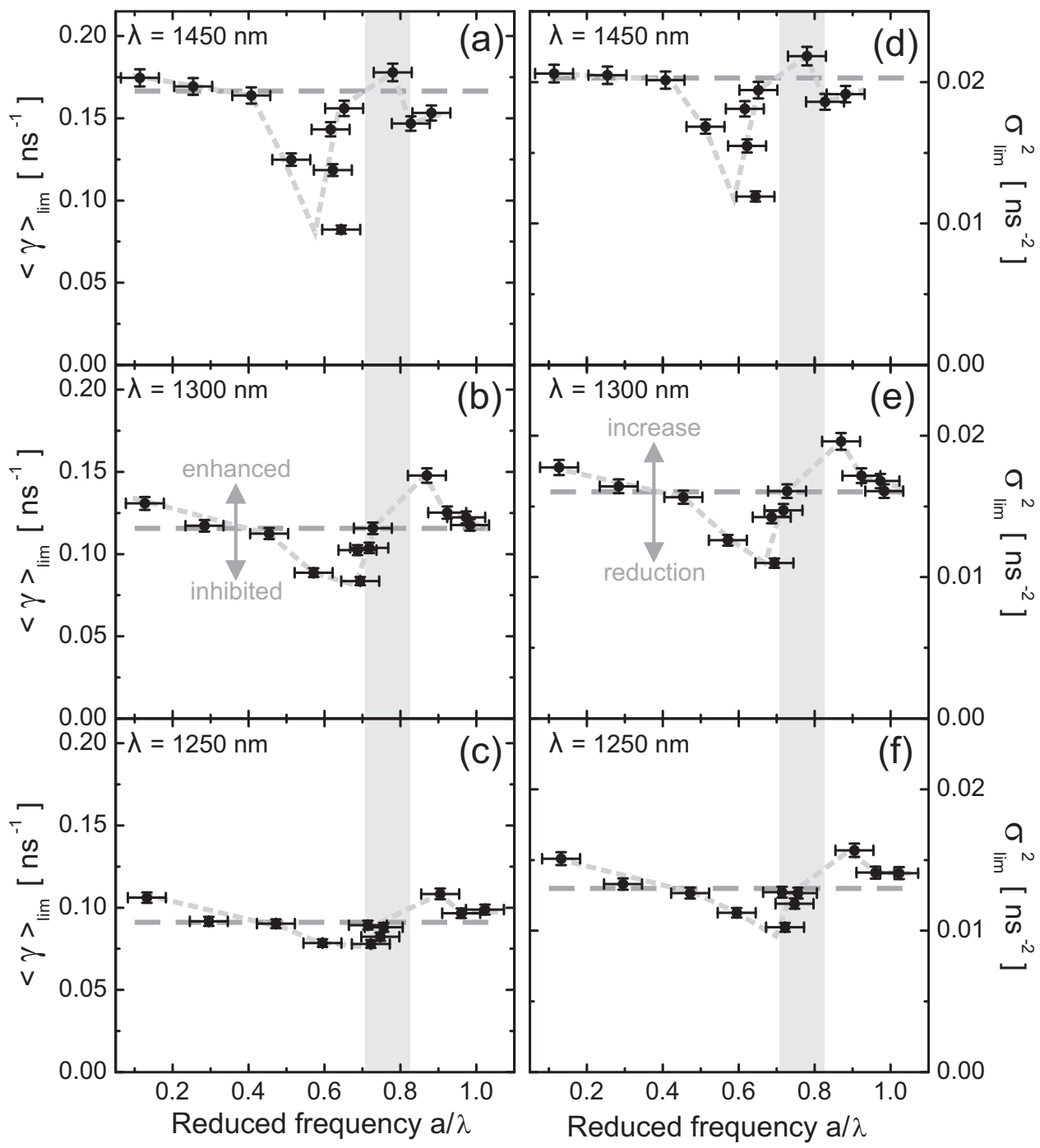

Figure 6.4: Averaged decay rate $\langle\gamma\rangle_{\lim }$ and variance $\sigma_{\lim }^{2}$ of colloidal PbSe quantum dot emission in titania inverse opals as a function of reduced frequency $a / \lambda$. (a,d) Results for $\lambda=1450 \mathrm{~nm}$, (b,e) $\lambda=1300 \mathrm{~nm}$, and $(c, f) \lambda=1250 \mathrm{~nm}$. The reduction of the average decay rate and variance around $a / \lambda \approx 0.77$ corresponds to the frequency of the crystals' pseudogap (gray area), where the local density of optical states is reduced. 


\begin{tabular}{ccccccccc}
\hline & \multicolumn{3}{c}{$\langle\gamma\rangle_{\lim }\left[\mathrm{ns}^{-1}\right]$} & & \multicolumn{3}{c}{$\sigma_{\lim }^{2}\left[\mathrm{~ns}^{-2}\right]$} \\
\cline { 2 - 3 } \cline { 6 - 8 }$\lambda[\mathrm{nm}]$ & $a / \lambda<0.45$ & $\min$. & $\max$. & & $a / \lambda<0.45$ & $\min$. & $\max$. \\
\hline 1250 & 0.091 & 0.078 & 0.11 & & 0.013 & 0.010 & 0.016 \\
1300 & 0.115 & 0.084 & 0.15 & & 0.016 & 0.011 & 0.020 \\
1450 & 0.167 & 0.082 & 0.18 & & 0.020 & 0.012 & 0.022 \\
\hline
\end{tabular}

Table 6.1: Overview of selected datapoints from Figure 6.4. At each measured emission wavelength $\lambda$ three characteristic values are shown for the average decay rate $\langle\gamma\rangle_{\lim }$ and the variance $\sigma_{\lim }^{2}$. These values are: 1) the long wavelength limit, 2) the minimum value, and 3) the maximum value. The long wavelength limit is set to the average value of the second and third datapoint at the low frequency side of the figures. The first point is disregarded because a change in the effective refractive index is expected, as explained in the text.

\begin{tabular}{cccccc}
\hline & \multicolumn{2}{c}{$\langle\gamma\rangle_{\lim }$} & & \multicolumn{2}{c}{$\sigma_{\lim }^{2}$} \\
\cline { 2 - 3 } \cline { 5 - 6 }$\lambda[\mathrm{nm}]$ & enhancement $[\%]$ & inhibition [\%] & & enhancement [\%] & inhibition [\%] \\
\hline 1250 & 19 & 14 & & 21 & 21 \\
1300 & 29 & 27 & & 22 & 31 \\
1450 & 6.7 & 51 & & 7.3 & 41 \\
\hline
\end{tabular}

Table 6.2: Overview of enhancement and inhibition of average decay rate $\langle\gamma\rangle_{\lim }$ and variance $\sigma_{\text {lim }}^{2}$ with respect to long wavelength range values. Used data from Table 6.1 as input. These inhibition and enhancement factors in the total decay rate and variance, are a lower limit for the inhibition and enhancement of the radiative part of the decay.

values for $\langle\gamma\rangle_{\lim }$ are listed in Table 6.1. In Table 6.2 the enhancement and inhibition factors with respect to the long wavelength limit are summarized. The maximum enhancement of $29 \%$ is observed at wavelength $\lambda=1300 \mathrm{~nm}$, and the maximum inhibition of $51 \%$ is observed at wavelength $\lambda=1450 \mathrm{~nm}$. The changes in $\langle\gamma\rangle_{\lim }$ are very significant, being much larger than the $3 \%$ uncertainty in the value of $\langle\gamma\rangle_{\lim }$, and several times larger than any changes that may arise from temporal effects on the decay curve (Section 6.3.2). Hence, the results from Figures 6.4.(a-c) clearly demonstrate the control of the spontaneous emission decay rate of quantum dots at near-infrared wavelengths using photonic crystals.

If the $\langle\gamma\rangle_{\text {lim }}$ data in Figures 6.4.(a-c) are studied as a function of wavelength at a fixed lattice parameter, it seems that the reduced frequencies of maximum enhancement and inhibition become larger for shorter emission wavelengths. The apparent frequency shift is currently not understood. The combination of these figures also show a clear increase of the decay rate with emission wavelength. This 
increase is attributed to the increase of the quantum dots' oscillator strength with the emission wavelength, as discussed in Section 3.4.

From the measured decay rates it is possible to estimate the photoluminescence quantum efficiency QE of the dots. For simplicity, the quantum efficiency pertaining to single-exponential decay is applied. Hence, the quantum efficiency is the ratio of the radiative decay rate $\gamma_{\mathrm{rad}}$ and the total decay rate $\gamma_{\text {tot }}$, as obtained from the experiment. The radiative decay is given by the total decay rate minus the non-radiative decay rate $\gamma_{\text {nrad }}$. Hence,

$$
\mathrm{QE}=\frac{\gamma_{\mathrm{rad}}}{\gamma_{\mathrm{tot}}}=\frac{\gamma_{\mathrm{tot}}-\gamma_{\mathrm{nrad}}}{\gamma_{\mathrm{tot}}} .
$$

As with photonic crystal the radiative decay of a light source can be changed, the crystal effectively also controls the quantum efficiency of the light source. To estimate the maximum quantum efficiency, the maximum value of $\langle\gamma\rangle_{\text {lim }}$ is used for $\gamma_{\text {tot }}$ (see Table 6.1). Hence, the maximum quantum efficiency is found in the dots that emit at $\lambda=1450 \mathrm{~nm}: \mathrm{QE}=(0.18-0.081) / 0.18=55 \%)$. This efficiency is a lower bound as the non-radiative decay rate is overestimated. The value is in good agreement with literature $[68,101,111,129,136,143,144]$. A more elaborate analysis requires a detailed study on quantum efficiencies of ensembles that exhibit non-exponential decay as in Reference [226].

Figures 6.4.(d-f) show the variances $\sigma_{\lim }^{2}$ of the distributions used to model the decay rates. The variance shows similar behavior as the average decay rate: for $a / \lambda<0.45 \sigma_{\lim }^{2}$ is practically constant, around $a / \lambda=0.6$ the variance is reduced, and an increase in the variance is observed around $a / \lambda=0.85$. Also, the variance increases with wavelength. For convenience, the measured maximum, minimum, and long wavelength values for $\sigma_{\lim }^{2}$ are listed in Table 6.1. A reduced variance corresponds to a reduced width of the distribution used to model the decay curve. Consequently, the decay curves are less non-exponential at lower variances [33].

\subsubsection{Comparison with experimental results from the litera- ture}

In this section our experimental results are compared to previous experiments that demonstrate spontaneous emission decay control using photonic crystals. First, experimental results in the visible range are considered of quantum dot emission from similar titania inverse opals. Second, an overview is given of other experiments at wavelengths $\lambda>1100 \mathrm{~nm}$.

The first demonstration of spontaneous emission control of light sources using photonic crystals was given in Reference [30]. There, the decay rate of CdSe quantum dots was enhanced and inhibited using titania inverse opals. These quantum dots emit in the visible range at wavelengths around $\lambda=615 \mathrm{~nm}$. The measured decay was very non-exponential, with the curvature determined by the photonic crystal, see also [14, 33]. The data were interpreted using the same log-normal dis- 


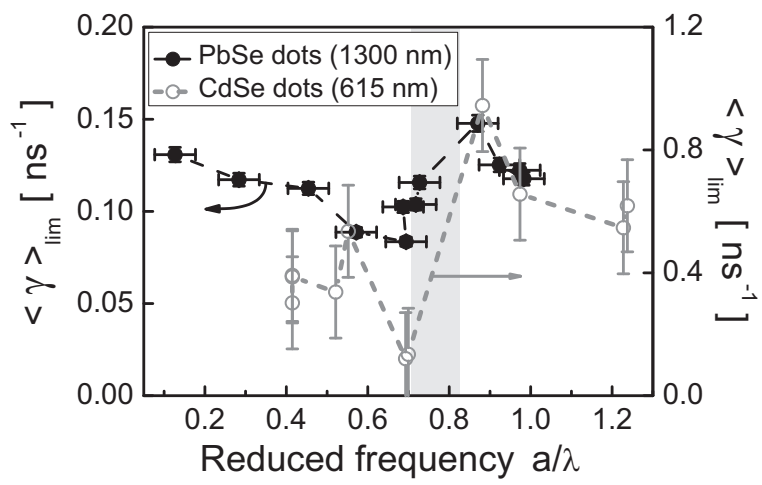

Figure 6.5: Average decay rate $\langle\gamma\rangle_{\text {lim }}$ of $\mathrm{PbSe}$ and CdSe quantum dots inside titania inverse opals versus reduced frequency $a / \lambda$. Closed dots are the PbSe data, measured at near-infrared wavelength $\lambda=1300 \mathrm{~nm}$. Open circles are the CdSe data, measured in the visible range, at wavelength $\lambda=615 \mathrm{~nm}$. The minimum and maximum value for $\langle\gamma\rangle_{\lim }$ occur at the same $a / \lambda$, for both data sets. The relative change of $\langle\gamma\rangle_{\lim }$ for different $a / \lambda$ is larger for CdSe quantum dots. As expected, the CdSe quantum dot decay is faster than the PbSe decay. The dashed lines are guides to the eye, and the gray area around $a / \lambda=0.77$ indicates the pseudogap region. Arrows point towards the ordinate that corresponds to the data. The error bars from the CdSe data are estimated from the largest difference between data on samples with similar lattice parameter $a$. The CdSe data were taken from [14].

tribution of decay rates, as applied in this chapter. Using these data, the average decay rate and standard deviation were determined and compared to the new $\mathrm{PbSe}$ quantum dot results.

Figure 6.5 shows the average decay rates $\langle\gamma\rangle_{\text {lim }}$ of CdSe and PbSe quantum dots in titania inverse opals versus reduced frequency. The average decay rate of CdSe quantum dots is several times larger than the average decay rate of PbSe quantum dots. This is explained by the larger oscillator strength and emission frequency of the CdSe dots $[132,162]$. In the long wavelength limit both data sets show approximately constant values for $\langle\gamma\rangle_{\text {lim. }}$. Spontaneous emission inhibition is observed for both data sets around $a / \lambda=0.65$. Furthermore, an enhancement of the decay rate is seen at $a / \lambda \approx 0.85$. For higher frequencies $\langle\gamma\rangle_{\text {lim }}$ decreases. Hence, the PbSe quantum dot results for $\langle\gamma\rangle_{\lim }$ are very similar to the CdSe quantum dot data. This is a very important result as it confirms the validity of our results and shows that the titania inverse opals can be used both in the visible and in the near-infrared range.

In Figure 6.6 the standard deviations $\sigma_{\lim }$ of the PbSe and CdSe quantum dot decay rate distributions are compared. Both types of quantum dots show the same qualitative behavior. The $\sigma_{\lim }$ values of CdSe quantum dots are larger than the values for PbSe dots. The $\sigma_{\lim }$ data resemble the $\langle\gamma\rangle_{\lim }$ data from Figure 6.5: all 


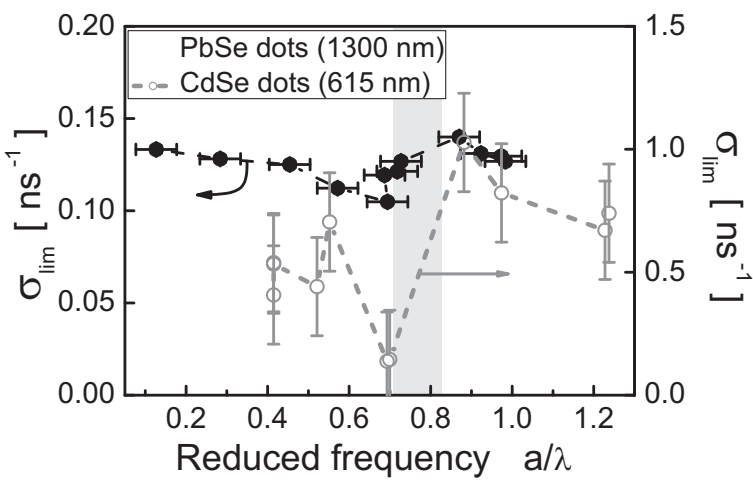

Figure 6.6: Standard deviation $\sigma_{\lim }$ of $\mathrm{PbSe}$ and $\mathrm{CdSe}$ quantum dots inside titania inverse opals, vs reduced frequency $a / \lambda$. Closed dots are the PbSe data, measured at near-infrared wavelength $\lambda=1300 \mathrm{~nm}$. Open circles are the CdSe data, measured in the visible range, at wavelength $\lambda=615 \mathrm{~nm}$. Both data sets show: (i) a reduced $\sigma_{\lim }$ at $a / \lambda=0.69$, (ii) a maximum $\sigma_{\lim } a / \lambda=0.87$, and (iii) practically constant $\sigma_{\lim }$ in the long wavelength limit. The error bars from the CdSe data are estimated from the largest difference between data on samples with similar lattice parameter $a$. The CdSe data were reproduced from [14]. The gray bar represents the pseudogap region, and arrows point towards the ordinate that correspond to the data.

data sets show constant values in the long wavelength limit, a decrease at reduced frequencies close to $a / \lambda=0.69$, and a maximum around $a / \lambda=0.87$. Hence, also the $\sigma_{\text {lim }}$ behavior determined from PbSe quantum dot experiments at near-infrared wavelengths are similar to the CdSe quantum dot data that were measured in the visible range.

In contrast to our results, the CdSe quantum dots do not show a spontaneous emission rate increase with emission wavelength. For CdSe nanocrystals the spontaneous emission rate is inversely proportional to the emission wavelength [162, 167, 173]. The different dependence of the emission rate on emission wavelength for CdSe and PbSe quantum dots does not affect the comparison made in Figures 6.5 and 6.6. This difference does underline the importance of comparing only results measured at the same emission wavelength.

Using photonic crystals, the first spontaneous emission inhibition at near-infrared wavelengths $\lambda>1100 \mathrm{~nm}$ was clearly demonstrated with quantum wells [31, 32]. These experiments were done at cryogenic temperatures of $T=4 \mathrm{~K}$. The authors stressed the ability to redistribute light emission using their photonic crystals. Surprisingly, the importance of the near-infrared wavelengths used was not explicitly mentioned. Note that quantum wells are very different from the quantum dot emitters. An exciton polariton in a quantum well is only confined in one dimension, whereas in quantum dots the exciton is confined in all three dimensions. Therefore, a quantum well can not be considered as a fixed dipole emitter [243]. 
In subsequent experiments the same group used InAs quantum dots to demonstrate inhibition of the spontaneous emission decay using a photonic crystal slab [32, 244]. However, only two decay curves were compared: one measured from a slab without photonic crystal, and one measured from a photonic crystal slab. As discussed in Section 6.2, comparison of decay curves in different environments can be misleading. First of all the effective refractive indices of these systems are not the same. The observed decay rate reduction in the photonic crystal may therefore be ascribed to the reduced refractive index. Secondly, no photonic crystal parameter is varied to demonstrate changes to the decay rate due to photonic crystal properties.

Control of spontaneous emission rates at wavelengths $\lambda>1100 \mathrm{~nm}$ was also claimed in 3D photonic crystals $[83,218,240,241]$. Clearly these papers are related to the work presented in this thesis, and show very promising results. As discussed below, however, these experiments do not satisfy all requirements (Section 6.2) needed to unambiguously claim spontaneous emission control by means of the photonic crystal. In Reference [83] a (single) decay curve measured from a erbium-doped silicon woodpile structure is compared to a decay curve measured from bulk erbium-doped silicon. The spontaneous emission rate in the photonic crystal was reduced by $25 \pm 10 \%$. An emission inhibition of $20 \%$ was reported from decay curves measured from PbSe quantum dots that were located inside a polymeric photonic crystal at distances of approximately 0,5 , and $10 \mu \mathrm{m}$ from the crystals' surface [241]. In Reference [240] the authors reported an inhibition of $35 \%$ and an enhancement $8.5 \%$ of PbSe quantum dot spontaneous emission rates, as a function of the detection angle with respect to the samples' surface. In this last paper [240], PbSe quantum dot decay curves measured from polymeric woodpile photonic crystals are compared to decay curve measurements on a 0.5 to $1 \mu \mathrm{m}$ thick quantum dot film. In these three papers $[83,240,241]$ the emitter in the reference sample is effectively in an environment with a different refractive index and should not be compared to the measurement in the photonic crystal. Furthermore, no photonic crystal parameters, like its lattice constant, were changed to demonstrate the influence of the crystals' periodicity on the decay rate. In Reference [240], the angular dependence of the lifetime is surprising, since there is no angular dependence in Fermi's Golden Rule.

Finally, spontaneous emission control was claimed using photonic crystals with different lattice parameters [218]. The samples consist of PbSe quantum dots in polymeric woodpile photonic crystals, see also [240, 241]. The authors claim a $14 \%$ inhibition of spontaneous emission decay which is inconsistent with their estimation of the dots' quantum efficiency of only $3.7 \%$. Unfortunately, the error bars in the experiment were not explained. Additionally, decay curves measured at different emission frequencies are compared, which can be misleading, as discussed in Section 6.2. A light brown coloring of the sample due to the quantum dots is mentioned, which suggests that re-absorption processes may influence the measurements. The quantum dot density cannot be inferred from the paper nor were reabsorption processes discussed. 
The counting statistics in Reference [218] warrant additional attention. In our experience on near-infrared time correlated single photon counting, the good signal to noise ratio retrieved at the low $(1 \mathrm{kHz})$ repetition rates used is highly surprising. Just like we do, the authors use a near-infrared photomultiplier detector from Hamamatsu. These detectors have a dark-signal countrate of approximately $200 \mathrm{kHz}$. This rate is 200 times larger than the laser repetition rate used in Reference [218]. As discussed in Section 5.2.2, good counting statistics is obtained for a photo-emission detection rate 100 times smaller than the laser repetition rate. Unfortunately, the excitation power and integration times were not mentioned. Furthermore, the first part of the decay curves was not shown, neither is it clear if time $=0 \mu \mathrm{s}$ corresponds to the arrival time of the excitation pulse. As demonstrated in Section 5.2.2 these excitation conditions are very critical for good counting statistics.

Ergo, we believe to be the first to demonstrate the control of spontaneous emission decay of quantum dots at near-infrared wavelengths using photonic crystals. In this wavelength regime, our experiments also happen to be the first to show inhibition and enhancement of spontaneous emission, using photonic crystals at room temperature, in contrast to the quantum well experiments in Reference [31] that were done at a temperature of $4 \mathrm{~K}$.

\subsubsection{Comparison with theory}

The radiative decay rate of a single dipole emitter is given by Fermi's Golden Rule (Equation 1.2), which contains the LDOS experienced by the emitter. The LDOS inside a photonic crystal can be calculated [14, 28, 29, 33, 242, 245]. However, it is not known which positions and orientations should be taken into account to calculate the LDOS of the isolated quantum dots in the ensemble measurement. In addition, these calculations are very time consuming. The position and orientation, unit cell average of the local density of states $\epsilon(r) N_{\mathrm{rad}}\left(a / \lambda, \mathbf{r}_{i}, \mathbf{e}_{\mu, i}\right)$ is the density of states $\operatorname{DOS}(a / \lambda)$. The DOS is relatively easy to calculate and well known for titania inverse opals $[14,242]$. Clearly, variations in the DOS correspond to changes in the LDOS. Therefore, measured average decay rates can be compared qualitatively to the DOS. As discussed previously, the measured average decay rate contains a radiative part and a non-radiative part. As the measurements are done at the same wavelength, the non-radiative part is expected to be constant for the measured range of reduced frequencies. Hence, for each measurement the long wavelength result of the mean decay rate is subtracted to find the radiative change of the decay rate, i.e., $\langle\gamma(a / \lambda)\rangle_{\lim }-\langle\gamma\rangle_{\text {hom }}$ is considered. To use the same approach for the calculations, the DOS in the long wavelength limit needs to be subtracted from the DOS at a given reduced frequency, or $\operatorname{DOS}(a / \lambda)-\mathrm{DOS}_{\mathrm{hom}}$.

Figure 6.7 shows the band structure (a), and the corresponding DOS (b) calculated for a titania inverse opal [242]. The pseudogap region in the DOS corresponds to frequencies in and just above the L gap shown in the band structure diagram. The $\operatorname{DOS}(a / \lambda)$ and $\operatorname{DOS}_{\text {hom }}$ are given by the open circles and gray line, respectively. 

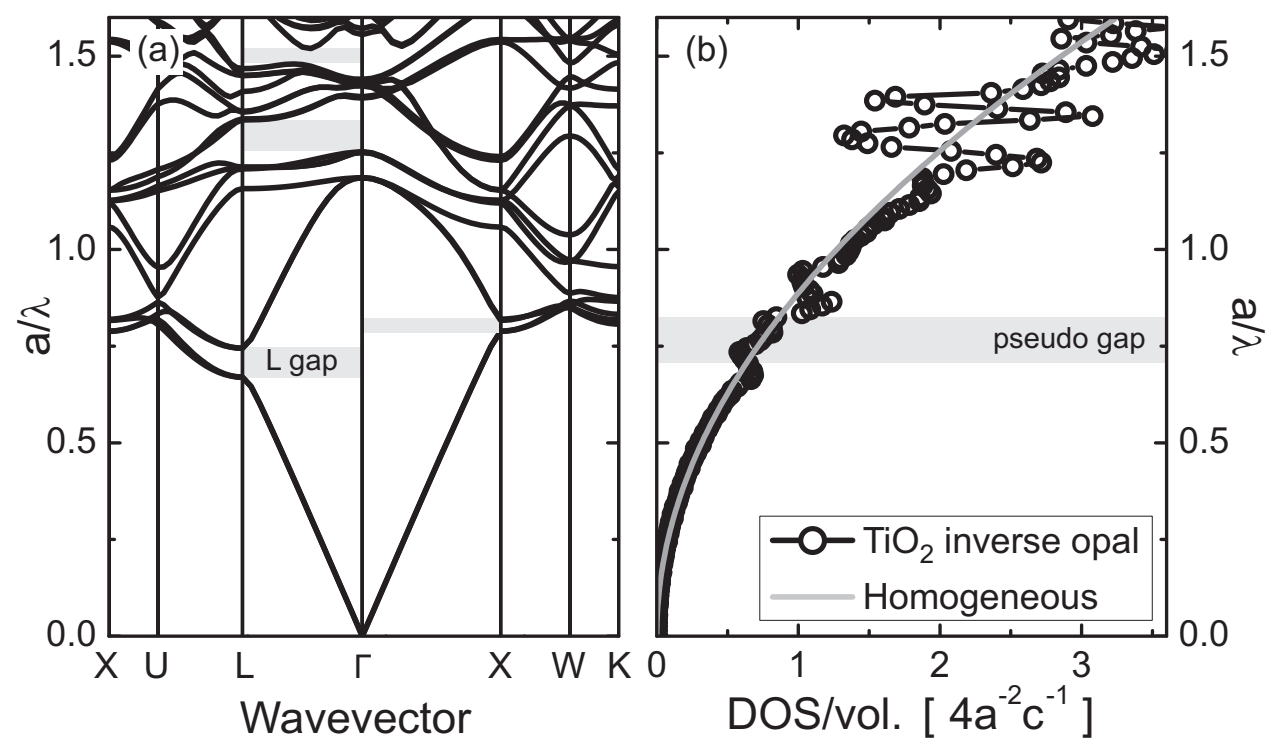

Figure 6.7: (a) Calculated band structure diagram of a titania inverse opal. Gray areas correspond to gap regions. (b) Calculated Density Of States for a titania inverse opal (open circles) and a homogeneous medium with effectively the same refractive index as the inverse opal (gray line). Gray area highlights the pseudogap region, where the opals' DOS deviates from the homogeneous medium. At higher frequencies $(a / \lambda \approx 1.3)$ the relative difference between the DOS of the opal and the homogeneous medium increases. The black line that connects the circles acts as a guide to the eye. The data is taken from [242].

Peaks and troughs are observed in the DOS of the titania inverse opal, whereas the DOS inside a homogeneous medium show the expected squared-frequency dependence.

In Figure 6.8 the experimental changes in $\langle\gamma(a / \lambda)\rangle_{\lim }$ are compared to changes in the $\operatorname{DOS}(a / \lambda)$ of the inverse opal. Decay curve data measured at a wavelength $\lambda=1300 \mathrm{~nm}$ are used. Furthermore, a homogeneous rate of $\langle\gamma(\lambda)\rangle_{\mathrm{hom}}=0.115 \mathrm{~ns}^{-1}$ is assumed, based on the average of the data measured at $a / \lambda=0.28$ and $a / \lambda=0.45$ (see Figure 6.4.(b)). Both theory and experiments show variations in the results, as a function of reduced frequency $a / \lambda$. The measurements reveal a minimum decay rate around $a / \lambda=0.70$. Especially at $a / \lambda=0.59$ the calculation deviates from the measurement, as the latter shows clear inhibition. However, experimentally there is no reason to doubt this measurement. The increase in the measured decay rate at $a / \lambda=0.13$ is attributed to bulk titania that covered the crystal very close to the measured location, as found out by a microscopy study. The additional titania causes an increase in the refractive index $n$ and consequently the emission decay rate increases [14]. The gray area denotes the calculations' pseudogap region, where 


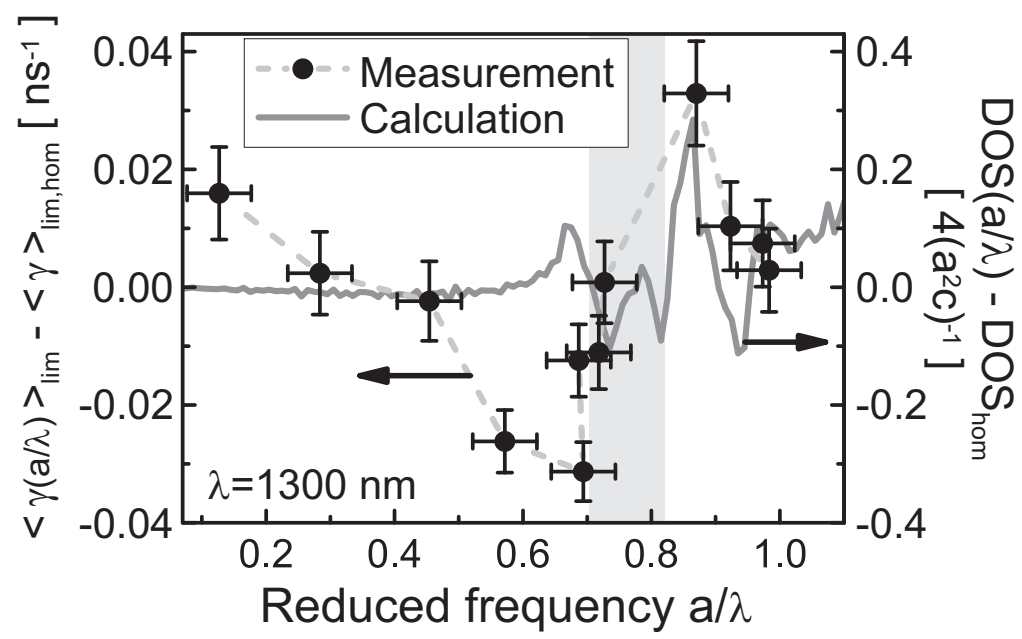

Figure 6.8: Measured average decay rate and calculated DOS changes from the long wavelength limit are plotted versus the reduce frequency. Symbols correspond to the measured $\langle\gamma\rangle_{\text {lim }}$ data. Gray, solid line corresponds to the DOS calculation. Dashed line is a guide to the eye. The measurement reveals a minimum at $a / \lambda \approx$ 0.70 , and the center of the calculated pseudogap region (gray area) lies at $a / \lambda=$ 0.77. Both experiment and calculation show an enhancement at $a / \lambda=0.87$ and tend to zero in the long wavelength limit $a / \lambda<0.45$. Arrows point towards the corresponding ordinate. Calculations stem from [242].

the decay rate is expected to be reduced.

\subsection{Conclusions}

We have presented the first demonstration of spontaneous emission control of dipole quantum emitters at near-infrared wavelengths $\lambda>1100 \mathrm{~nm}$, using photonic crystals. The results were reproduced for three different wavelengths. A maximum enhancement of $29 \%$ was observed for the measured average total decay rate. In addition, the measured average total decay rate was inhibited by up to $51 \%$. These numbers are a lower limit of the changes in the radiative decay rate of the quantum dots. This is the first time spontaneous emission rates are controlled at near-infrared wavelengths using 3D photonic crystals. Furthermore, these results happen to be the first to show spontaneous emission control of near-infrared light sources, using photonic crystals at room temperature.

To our knowledge, this is the first time PbSe quantum dots are controlled by means of the Local Density Of States. The importance of comparing only measurements taken at the same emitter wavelength was demonstrated. Our near-infrared results show similar results as experiments in the visible range where CdSe quantum 
dots were used with the same crystals. Consequently, the titania inverse opal photonic crystals can be applied over a broad frequency range. Furthermore, the results are in qualitative agreement with a model, based on Density Of States calculations.

The near-infrared wavelength range, addressed in this chapter, is of interest for the telecom industry and for photonic band gap experiments. Here, we provided a possible light source together with the measurement procedures needed for future quantum optics research in the near infrared. 



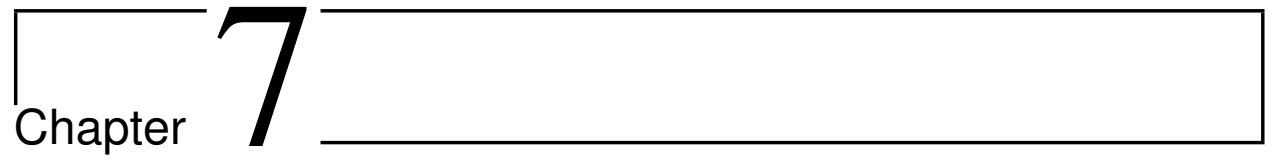

\section{Realignment to nanostructures in different experimental setups}

\subsection{Introduction}

Nanotechnological developments are at the base of new applications in electronics, quantum information processing, and nanophotonics. Many different nanometer sized structures have been fabricated and isolated for elaborate study [91, 246-249]. An important class of nanostructures are so-called metamaterials [250]. These composite materials exhibit properties that depend in particular on their geometrical structure, like for instance the inverse opals used in Chapters 4 to 6 . The physical properties of nanostructures like metamaterials, single molecules, and carbon nanotubes not only depend on their detailed structure but often also on their immediate vicinity. For experimental observations it is therefore very important to be able to prepare a pre-defined structure and subsequently probe exactly that same structure.

In this chapter we are specifically interested in structures for nanophotonics. A photonic sample is considered in which nanometer sized regions of interest are fabricated, as depicted in Chapter 1. These nanostructures need to be examined in separate experimental setups. The various setups yield complementary information on the individually targeted nanostructures, e.g., structural and optical reflectivity data. The ability to perform these experiments requires a deterministic approach to position and orient the structure with respect to the different experimental setups. Coordinates that can be obtained from translation stages in the setup are not sufficient to determine the exact position of the nanostructures under study. Therefore additional control is needed.

In the semiconductor integrated circuit industry, position control of known structures is obtained by the creation of special markers on the wafers. These markers 
provide a firm alignment grid that is needed in the different fabrication steps of, e.g., multilayered structures [251]. The grid is also used to recover a certain position or nanostructure on a sample, see for example Reference [252]. Unfortunately, the process of artificial grid fabrication involves extensive and expensive production facilities that are not generally accessible. Furthermore, these grids may damage the structure. Last but not least, grid fabrication is impossible if a sample needs to be created by self-assembly only. In that case, the region of interest is not a-priori known.

Here, a different approach to recover the same region of interest is demonstrated. The approach can even be applied to samples with a low electrical conductance. Intrinsic geometrical features in the vicinity of a fabricated nanostructure are used to act as position unique markers. These markers can be recovered in the images obtained from different experimental setups by use of, e.g., cross-correlation techniques.

The following Section 7.2 contains a detailed description of the experimental methods, followed by results and discussions. As an illustration, surface cavities with radii of $65 \mathrm{~nm}$ on a silica opal photonic crystal are probed with various setups: a focused ion beam workstation, a scanning electron microscope, a wide field optical microscope, and a confocal microscope. Cross-correlation techniques are used to recover a small area imaged with the scanning electron microscope in a large area photographed with the optical microscope. This provides a possible avenue to automatic searching. It is shown how both structural and optical reflectivity data can be obtained from one and the same nanostructure. Subsequently, the realignment procedure is applied in a study of opal layer growth, Section 7.4. This shows the simplicity of the method in a practical situation. Conclusions form the last part of this chapter, Section 7.5.

\subsection{Realignment method}

The structures under study consist of arrays of point defects. These arrays are first milled on the surfaces of both polystyrene and silica opal photonic crystals [253]. The opals with sphere radii of $111 \mathrm{~nm}$ (polystyrene) and $113 \mathrm{~nm}$ (silica) are grown by self-assembly on a glass microslide substrate by use of the vertical controlled drying method [254]. Prior to milling, carbon was deposited on the surface of the opal with a Bio-Rad E6700 Turbo Coater operating at a current of 50 A. Carbon layers have typical thicknesses of about $10 \mathrm{~nm}$ and assist in removing charges from the milled area.

To fabricate the nanostructures the carbon coated opal is subsequently placed inside a FEI "xT Nova Nanolab 600" focused ion beam workstation at MESA ${ }^{+}$. Use is made of a gallium ion beam accelerated at $30 \mathrm{kV}$ and a beam current of $9.7 \mathrm{pA}$. The focus of the ion beam had a diameter of about $20 \mathrm{~nm}$. The defects were milled with the ion beam by writing circular patterns with a diameter of $120 \mathrm{~nm}$, positioned on the center of the sphere. Arrays were milled intermittently. This means that 


\begin{tabular}{|c|c|c|c|c|}
\hline \multirow[b]{2}{*}{ Apparatus } & \multicolumn{2}{|c|}{$\begin{array}{l}\text { Magnification } \\
\text { range }\end{array}$} & \multirow{2}{*}{$\begin{array}{c}\text { Lateral } \\
\text { resolution } \\
{[\mathrm{nm}]}\end{array}$} & \multirow{2}{*}{$\begin{array}{c}\text { Acceleration } \\
\text { voltage used } \\
{[\mathrm{kV}]}\end{array}$} \\
\hline & $\min$ & $\max$ & & \\
\hline FIB & $37 \mathrm{x}$ & $1,280,000 \mathrm{x}$ & 7 & 30 \\
\hline SEM & $40 \mathrm{x}$ & $1,000,000 \mathrm{x}$ & 3 & 1 to 1.5 \\
\hline Optical microscope & $5 \mathrm{x}$ & $100 \mathrm{x}$ & $10^{2}$ & - \\
\hline Home-built microscope & $40 \mathrm{x}$ & $40 \mathrm{x}$ & $10^{2}$ & - \\
\hline
\end{tabular}

Table 7.1: Overview of the apparatus used with their corresponding magnifications, lateral resolutions, and acceleration voltages.

between each milling step of 5 seconds, milling was stopped for approximately 10 seconds to promote redistribution of charges away from the processed area. Together with the applied carbon layers, this approach prevents charge build-up of the milled area which typically results in beam drift and substantial damage to the opal. All single point defects which are part of the array are milled in parallel.

It is advantageous to align the samples' substrate edges parallel to the $(\mathrm{x}, \mathrm{y})$ translation axes of the sample positioner as many experimental setups use translation stages. Spatial $(\mathrm{x}, \mathrm{y})$ coordinates relative to two corners of the substrate are used to identify the location where the material nanocavities are created with a precision of about $50 \mu \mathrm{m}$. The sample is monitored during the FIB process at a high magnification of typically 65,000 times, using an Everhart Thornley secondary electron Detector (ETD). This in-situ imaging uses the same acceleration voltage and beam current as mentioned above. The environment of the fabricated region of interest is mapped by taking lower magnification images (down to 312 times). See Table 7.1 for an overview of the available magnifications. The region of interest is kept in the center of the images in order to know its position in low magnification graphs that do not resolve the nanostructure. Intrinsic sample features that appear in these images, like grain boundaries in opals, are used to act as markers for subsequent alignment purposes.

After the fabrication process, the geometrical shape and arrangement of the realized material cavities is examined in detail. As the carbon layer may blur the fine details it is first removed using oxygen plasma from a Tepla $300 \mathrm{E}$ plasma processor, with typical parameters: 15 minutes duration, $300 \mathrm{~W}$ plasma power, 1.25 mbar pressure, and $50 \% \mathrm{O}_{2}$ flow. For high contrast imaging of this low-conductance sample it is placed in a LEO 1550 high resolution scanning electron microscope, operating at a low acceleration voltage ( 1 to $1.5 \mathrm{kV}$ ), a working distance of $3 \mathrm{~mm}$ and using the In-lens detector to collect secondary electrons for imaging. See Table 7.1 for the available magnifications.

Recovery of our region of interest requires the following steps. First the edges of the samples' substrate are carefully aligned parallel to the $(\mathrm{x}, \mathrm{y})$ translation axis of 
the sample positioner. Subsequently, the $(\mathrm{x}, \mathrm{y})$ coordinates relative to the substrate corners that were obtained during the FIB session are utilized to approach the region of interest to within about $50 \mu \mathrm{m}$. Using the SEM at a relatively low magnification (down to 84 times), the SEM pictures are visually compared to the images that were taken with the FIB. When intrinsic structural features on the surface of the sample are recognized in both the FIB and SEM images it is possible to align to the exact location of the region of interest. Spatial $(x, y)$ coordinates of the material nanocavities relative to two corners of the substrate are recorded. Subsequently, images at various magnifications are taken with the SEM in addition to the images from the FIB process. After recovery of the nanostructure it was studied in detail using higher magnifications (up to 266,000 times).

After the geometry has been examined we want to study optical properties of the material cavities. Two different optical microscopes are used, see Table 7.1. Bright field optical microscope imaging is performed with a Nikon Eclipse ME600L microscope and CFI LU Plan BD objectives. A DXM-1200F digital camera is equipped to this microscope for imaging purposes. To localize our region of interest we use the relative coordinates and images from the SEM session in combination with a low magnification (10x) objective. After a characteristic structural feature from the SEM images is recognized, we move the sample such that the region of interest is in the center of the image and study the sample optically with higher magnification objectives (up to 100 times).

Reflectivity measurements with a high spatial resolution are performed using a sample scanning confocal microscope built by the Biophysical Engineering Group at $\mathrm{MESA}^{+}$. An infinity corrected microscope objective (Olympus, 40x/0.60NA $\infty / 0-2 / \mathrm{FN} 22$ ) is used to focus white light on the sample and collect the reflected light. The reflected light is focused through a pinhole with a diameter of $25 \mu \mathrm{m}$ and subsequently directed to a spectrometer. In the spectrometer the light is dispersed by a glass prism and projected onto a liquid nitrogen cooled ccd detector (Princeton Instruments, Spec-10:100B). In front of the pinhole a flip mirror can be used to look at the sample with a ccd camera for alignment purposes. This results in an area of $40 \mathrm{x} 40 \mu \mathrm{m}^{2}$ that is imaged on a television screen. The sample is mounted on an (x,y) scanning piezostage (Physik Instrumente, P-740.20). A manual (x,y) stage is mounted on this piezostage to provide additional translation length. Using the relative $(\mathrm{x}, \mathrm{y})$ coordinates and images available from the FIB and SEM session we are able to recognize characteristic features like grain boundaries on the sample. As the location of the region of interest is known with respect to these features, we track the sample on the television screen while translating it such that the domain with the nanocavities comes into view.

After the alignment to the region of interest, the area reflectance of the samples' surface is collected by scanning the sample line by line through the focus of the objective using the piezostage. The measured reflectance spectra are divided by the reflectance measured from a broadband dielectric mirror (Thorlabs, BB1-E02) to obtain a reflectivity spectrum at every measured position. Additional software was 
written to select a wavelength range and represent the average reflectivity in this range as an area plot.

To calculate cross-correlations between two images standard Matlab (The MathWorks, Inc.) routines are used. Images with the same resolution are imported in Matlab and converted to grayscale using the 'rgb2gray' function. Subsequently the images are transformed to black and white images using 'im2bw', with a threshold value obtained by using 'graythresh' on the grayscale images. Finally the black and white images are inverted and used as input for the normalized cross-correlation function 'normxcorr2'.

\subsection{Results and discussion}

The principal results of our deterministic method to study the same region of interest in various experimental setups are combined in Figure 7.1. Figure 7.1.(a) shows an image taken with the FIB workstation during fabrication. The individual silica spheres in the opal are recognized and near the center one sees an array of seven milled spheres that are intended as a composite point defect. Before the sample leaves the FIB workstation, low magnification pictures are taken in order to map the surroundings of the nanostructure under study, see Figure 7.1.(b). Stressinduced cracks that arise during the drying process of the opal are recognized as the thick, black meandering grain boundaries [206, 254]. These boundaries delineate single-crystal domains with unique, characteristic shapes. The morphology of these grains cannot be predicted a-priori, which differs from structures in semiconductor industry that are known by design [92]. The shape of a grain boundary itself may be very distinct with respect to its vicinity because of its width or because it is relatively straight and running alongside many domains. For our opals these grain boundaries provide the guidance needed to recover the position and orientation of the milled spheres in other experimental setups.

After the fabrication process the sample is mounted in the SEM to study the detailed structure. Figure 7.1.(c) shows the low magnification SEM image that was taken to map the vicinity of the location to which we want to align. The domain from Figure 7.1.(b) is recognized and subsequently translated to the center of the SEM image. For clarity this domain is outlined by a black box. The material cavities manifest themselves as a single bright region, marked by the arrows. The location of the material nanocavities is now recovered and the SEM is subsequently used at higher magnifications. The resulting SEM image in Figure 7.1.(d) shows exactly the same nanocavities as Figure 7.1.(a). Clearly this SEM provides much sharper images and superior contrast compared to the images from the FIB station, making the SEM data superior with these low-conductance samples. For instance, the SEM image shows that several voids completely perforate the sphere in which they were milled, since one can see the next layer of spheres below. Similar results are obtained for the polystyrene opals (data not shown).

Once the samples' structure is elucidated by SEM, we wish to study the nanos- 


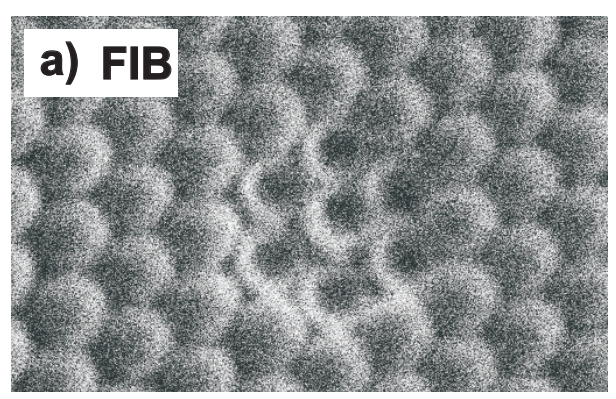

$500 \mathrm{~nm}$

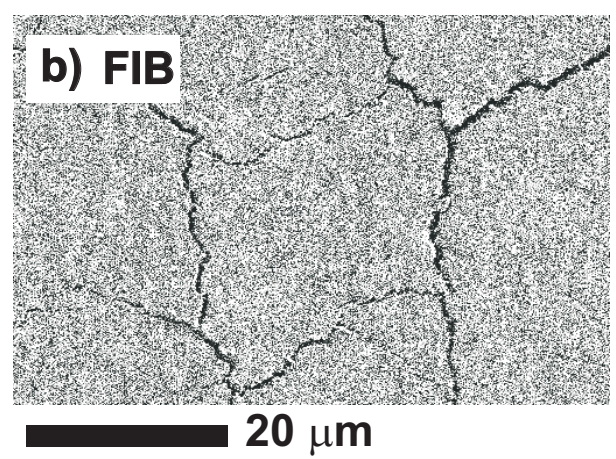

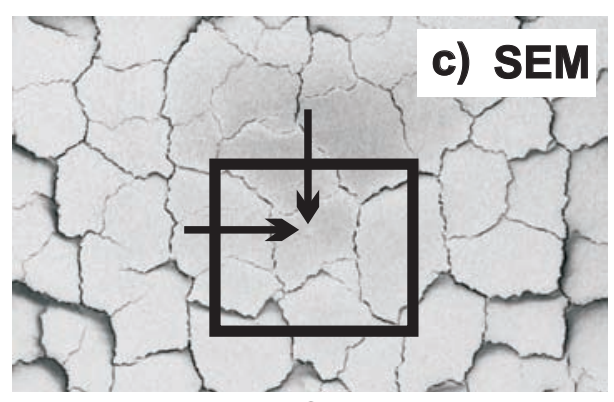

$50 \mu \mathrm{m}$

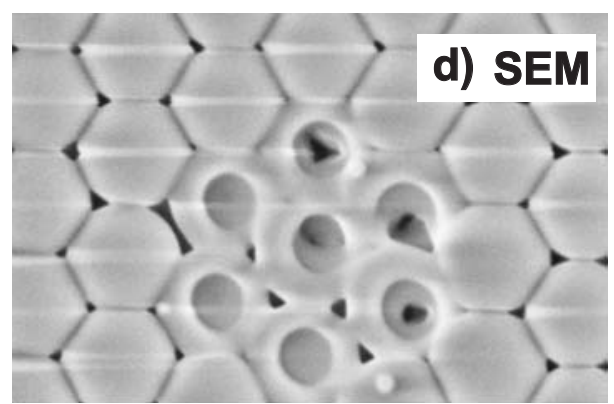

$500 \mathrm{~nm}$

Figure 7.1: (a) Micrograph obtained in-situ during focused ion beam milling using a magnification of 65,000 times. Individual silica spheres $(R=113 \mathrm{~nm})$ are clearly visible. Near the center of the image, holes are milled in seven spheres. To map the environment from these milled spheres the magnification of the focused ion beam workstation is reduced to 2,000 times. (b) Micrograph with the obtained map. After fabrication the sample is placed in a scanning electron microscope. (c) High resolution scanning electron micrograph at a magnification of 2,000 times. The area with the domain from (b) is recovered and highlighted by the black rectangle. The arrows point towards the milled defects. Stress induced grain boundaries in the sample show up as meandering lines which are useful for sample orientation and positioning in both (b-c). (d) High resolution scanning electron micrograph of the same hole array as shown in (a), depicted with a high magnification (266,000 times). The horizontal streaks and the non-roundness of the spheres are charging effects of the SEM caused by the low conductance of the samples' surface. The images are resized and reoriented for clarity.

tructure with a second, different setup such as an optical microscope. Figure 7.2 contains the optical microscope results. The bright-field image in Figure 7.2.(a) shows that the grain boundaries in the opal structure are visible in these optical images. The black box outlines the same crystal area as in Figure 7.1.(c). For this particular experiment, we found the material cavities by searching for the character- 

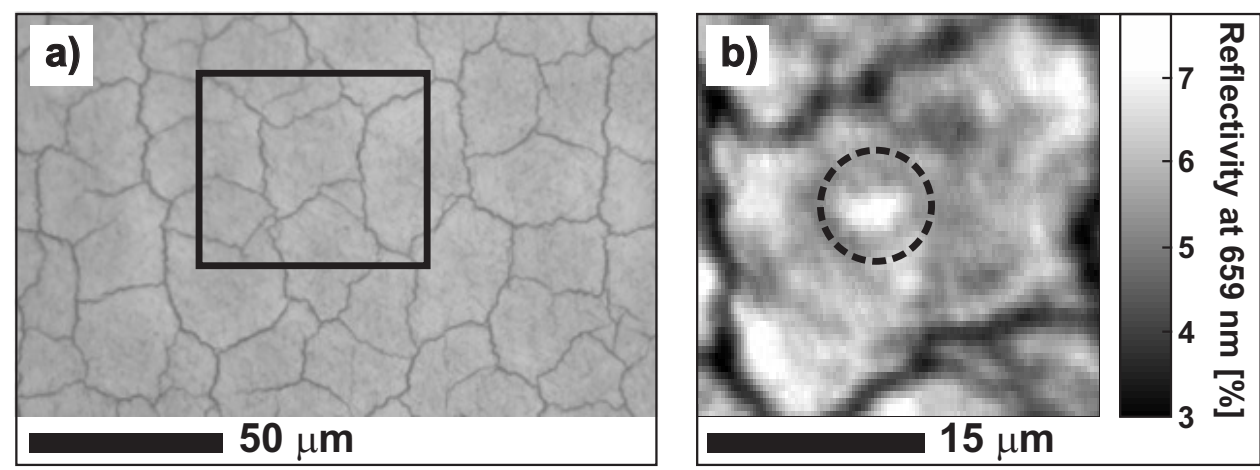

Figure 7.2: (a) Bright field optical microscope image of an opal of silica spheres (radius $r=113 \mathrm{~nm}$ ). The crystal domain that contains our region of interest is recognized by cross-correlating this image with the area inside the rectangle from Figure 7.1.(c). We outline the same area as a guide to the eye. The sample appears green due to the first order photonic stop band from the opal. (b) Reflectivity map for wavelengths in the range of 658.2 to $659.6 \mathrm{~nm}$, measured with the scanning confocal microscope in the crystal domain that contains the surface cavities. The location of the material nanocavities is encircled by the dashed line and the size of the pixels is $0.30 \times 0.60 \mu \mathrm{m}^{2}$. The reflectivity from the material cavities (about $8 \%$, white area) is higher than that of its vicinity.

istic small triangular domain at the bottom right, inside this black box. In practice, the sample looks green due to optical Bragg diffraction [255], in agreement with a measured photonic stop band centered at $514 \mathrm{~nm}$.

The map in Figure 7.2.(b) shows a high resolution spatial distribution of the optical reflectivity of the domain that contains the milled holes. This map was measured by confocal microscopy where the sample was scanned with a stepsize of $0.30 \mu \mathrm{m}$. To construct the graph the results for a range of wavelengths are collected from the measured spectra. We chose 658.2 to $659.6 \mathrm{~nm}$ which is a representative range corresponding to optical photon energies below the photonic stop band, hence the reflectivity is low, about 4 to $8 \%$. The crystal domain is recognized by its boundaries, where the reflectivity is lowest. Although the milled point defects are smaller than the spatial resolution of the experimental setup, the reflectivity from these nanostructures is distinct from its environment. For this wavelength the reflectivity from the nanostructures appears to be higher than that of its vicinity. For photon energies above the photonic stop band it appears that the reflectivity from the milled spheres is lower than that of the vicinity. Our results are in qualitative agreement with near-field scanning optical microscope experiments on opal surfaces with accidental point defects [77]. A detailed analysis of the results is outside the scope of this thesis.

For general usage, it would be convenient if our method could be extended 


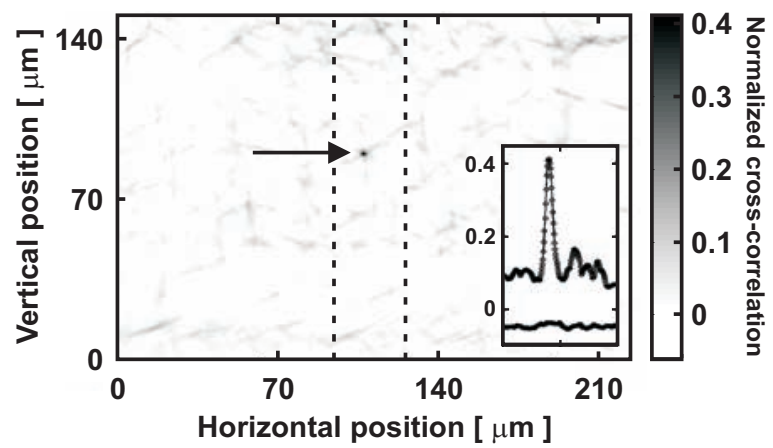

Figure 7.3: Result of the normalized cross-correlation between the area inside the rectangle of the SEM image of Figure 7.1.(c) and the optical microscope image of Figure 7.2.(a). A clear dark spot appears at the position where the two images show the best overlap. This spot is indicated by the arrow. The inset is a zoom-in for horizontal positions between 95 and $126 \mu \mathrm{m}$ (dashed lines). The upper data are the maxima and the lower data the minima on each horizontal position. The maximum of the correlation function is clearly peaked and its value stands out of the maximum background by a factor of three.

by automating it. Therefore, images obtained with the different setups are crosscorrelated. Figure 7.3 shows the result of a normalized two dimensional crosscorrelation between the area outlined in Figure 7.1.(c) and the full Figure 7.2.(a). A clear dark spot appears where the images overlap best. The inset shows the side view from the area between the dashed lines, and reveals that the location of best overlap clearly stands out of the maximum background by a factor of three. The full width at half maximum of this cross-correlation peak is $2.2 \mu \mathrm{m}$. After recovery of the region of interest with this precision one can increase the magnification to improve the alignment.

Let us briefly discuss limitations of the precision with which structures can be aligned in our setups. Similar limitations hold in general for all experimental setups. In the fabrication process with the FIB workstation, position uncertainties up to $50 \mu \mathrm{m}$ are introduced while choosing the position of the substrate corners and because the substrate edges may not be completely parallel to the (x,y) translation axes of the stage. Similar uncertainties hold for the SEM setup. Therefore, these coordinates are not sufficient to recover the same nanostructure. In the wide field microscope it appeared to be difficult to manually align the substrate parallel to the axes of the translation stage. Furthermore, these stages exhibit hysteresis and the accuracy of the rulers are two orders of magnitude less precise than the rulers used in the FIB and SEM. In case of the confocal microscope it is inconvenient that the area that can be monitored during alignment is small. All together, these alignment issues pose practical demands on the low magnification FIB and SEM images that 
are used to recover the nanostructures. For these opals we found maps that cover about $0.5 \times 0.5 \mathrm{~mm}^{2}$ to be very desirable.

Our method is straightforward and generally applicable in many research areas, especially in case of self-assembly and low-conductance samples. The method is very useful to align to different locations on a sample to reproduce experimental results. In biophysics one may use the present approach to study individual bacteria, viruses, molecules, or specific areas of cell-walls and cells in general [256, 257]. The attachment of wire bonds to nanostructures may also be established with the help of this method. As an illustration of its simplicity, the alignment method is used to study photonic crystal growth in the following section.

\subsection{Application of realignment procedure to study opal layer growth}

The alignment procedure, explained and demonstrated in the previous section will now be applied to study layer growth in opal photonic crystals. The ability to grow additional layers of spheres on the surface of an existing opal opens up new ways to fabricate samples for cavity quantum electrodynamics [19]. First, the vertical controlled drying method is used to grow an opal. Then, material cavities are created on the surface of this opal, as discussed in Section 7.2. Subsequently, additional layers of spheres are grown over the fabricated cavities. This way the material cavities become embedded inside a 3D photonic crystal, where they act as scattering centers, i.e., optical cavities.

To test steps in this fabrication procedure silica opals with sphere radii of $r=$ $113 \mathrm{~nm}$ are used to demonstrate the growth of an opal on top of an existing opal. Figure 7.4 shows the opal as grown on a glass microslide substrate. On the left is a schematic representation of the complete sample. Microscope images are stitched together to map out the complete opal. The boundary of the opal is marked. Below (above) this boundary there is only opal on glass (glass). The dotted line at the bottom of the figure denotes the area that is somewhat disrupted due to a glass rim that is needed to keep the substrate in place. The right side of Figure 7.4 shows a zoom in of a small region of the opal. The black, meandering lines are domain boundaries, similar to Figure 7.2.(a). As the sample surface is fully charted an additional opal can be grown on top, using the vertical controlled drying method once more.

Figure 7.5 shows a zoom in on a small region of the opal. Figure 7.5.(a) shows the photonic crystal surface of the original opal. Figure 7.5.(b) contains the same region as (a), after another vertical controlled drying step was applied to grow additional layers of spheres. The dashed ovals highlight regions where domain boundaries disappeared after the second growth step. The dashed box contains a domain boundary that became much more pronounced. Additional reflectivity measurements revealed that the sample became thicker in this region, as discussed below. 


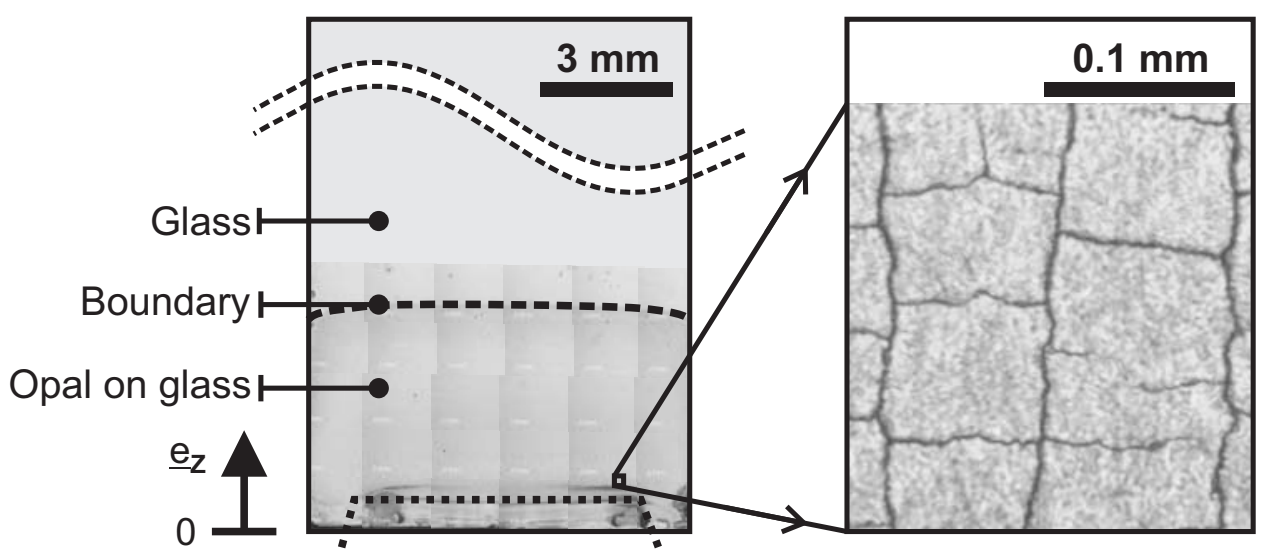

Figure 7.4: Left: microscope images stitched together to map out the silica opal. Dotted line in the bottom separates the region where opal growth is hindered by a rim that keeps the sample in place during the vertical controlled drying procedure. Right: zoom in on an area of the opal. Domain boundaries are clearly visible as the black meandering lines. Both images come with their own scalebar, depicted by the black bar.
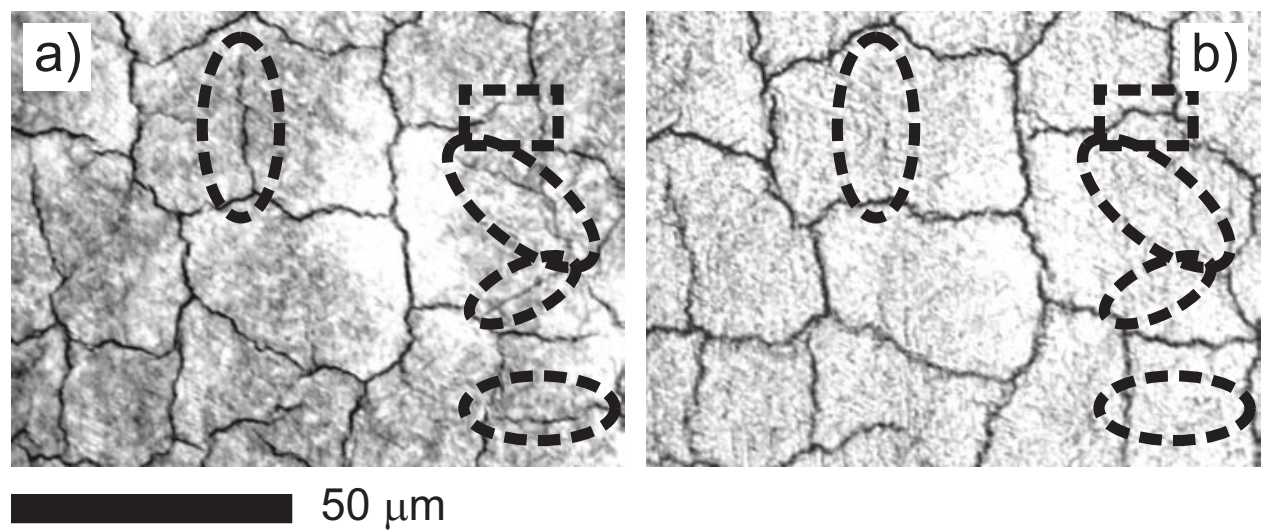

Figure 7.5: (a) Microscope image of an opal surface. Domain boundaries appear as black meandering lines. Subsequently, additional layers of spheres were grown over this opal. (b) Microscope image of the opal surface after the additional growth step. (b) Shows the same area as (a). Dashed lines indicate regions where domain boundaries disappeared (oval shaped) or became more pronounced (rectangular box) after the second growth step. The images are converted to grayscale and the contrast is manually enhanced for clarity. A joined scalebar is depicted at the bottom left of the figure. 

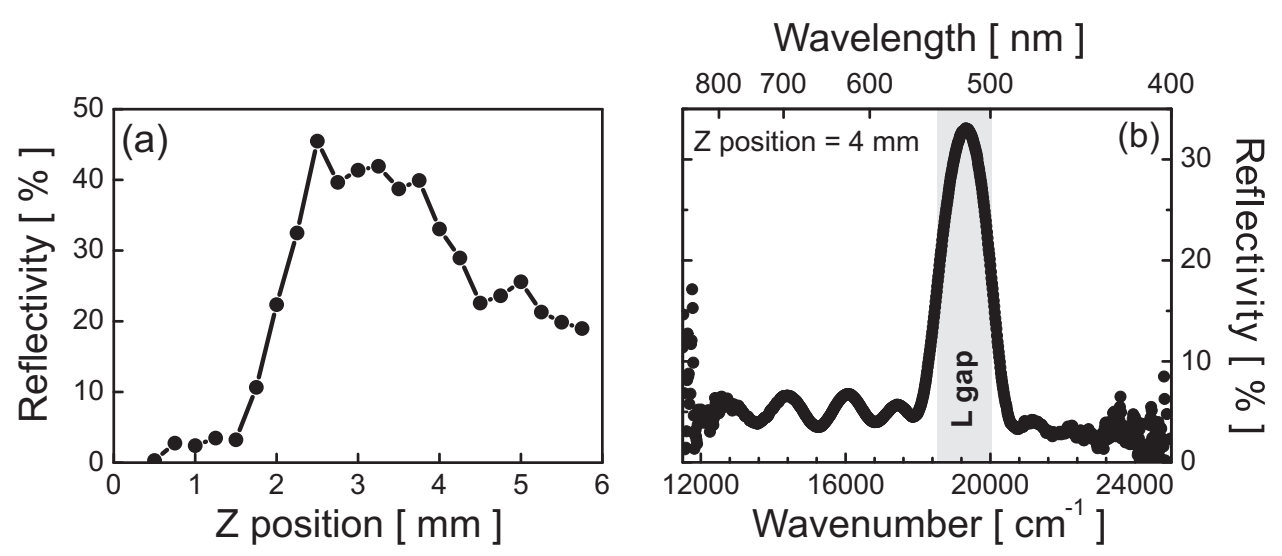

Figure 7.6: Results reflectivity measurements on silica opal, after a second opal growth step. (a) Reflectivity versus $Z$ position shows that the reflectivity is not constant over the samples' surface. Small $Z$ values yield hardly any reflectivity, while for too large $Z$ values the reflectivity decreases. The optimal $Z$ range is between 2.5 and $3.5 \mathrm{~mm}$, where the reflectivity is highest. The line connecting the dots is a guide to the eye. (b) Typical reflectivity results shows a clear Bragg peak, with Fabry-Pérot fringes on its sides. This measurement was done at $Z=$ $4 \mathrm{~mm}$. The Bragg peak wavenumber corresponds to the $L$ gap in band structure calculations (gray area).

Due to the disappearance of several domain boundaries, the domains appear larger for the thicker samples. This is in agreement with an elaborate study on these crystals [206].

Both the thickness and the quality of the photonic crystal can be retrieved from reflectivity measurements. Figure 7.6 summarizes the results of 22 reflectivity measurements, taken after the second opal growth step. The reflectivity was measured from points on a vertical line in the center of the opal. Each point has a different $Z$ value, see Figure 7.4. Figure 7.6.(a) shows clearly that the reflectivity value changes with $Z$ position. For $Z<2 \mathrm{~mm}$ no clear Bragg diffraction peak was observed. There, the growth process is hindered by a glass ridge that keeps the substrate in place. For $\mathrm{Z}$ values between 2.5 and $3.5 \mathrm{~mm}$ the reflectivity is maximum. For $Z>3.5 \mathrm{~mm}$ the reflectivity decreases. A typical reflectivity measurement is shown in Figure 7.6.(b). A clear reflectivity peak is observed, with smaller fringes at its sides. The largest peak is caused by Bragg diffraction and its wavenumber range corresponds to the $\mathrm{L}$ gap in band structure calculations. The smaller peaks are Fabry-Pérot fringes, caused by interference of the reflection from the samples' front surface and the substrate. From these fringes, the thickness $d$ of the sample can be calculated, using

$$
d=\frac{\Delta m}{2 n_{\mathrm{eff}} \Delta(1 / \lambda)} .
$$




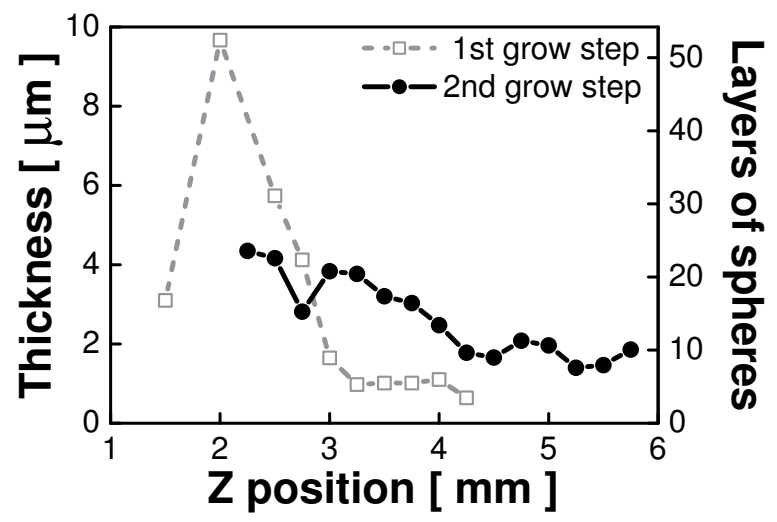

Figure 7.7: Measured opal thickness as function of $Z$ position. Open squares are the results after the first vertical controlled drying growth of the opal. Black dots show the results after a second vertical controlled drying growth step. For $Z<3 \mathrm{~mm}(Z>3 \mathrm{~mm})$ the photonic crystal becomes thinner (thicker). The lines are guides to the eye. From the thicknesses the number of layers of spheres was calculated (right vertical axis).

Here, $\Delta m$ is the number of fringes and $\Delta(1 / \lambda)$ is the change in wavenumber. Furthermore, $n_{\mathrm{eff}}$ is the volume averaged refractive index, which is assumed to be $n_{\text {eff }}=1.33$ for these silica opals.

The calculated thicknesses are combined in Figure 7.7. Both the thicknesses determined after the first and second growth step are depicted. As not all reflectivity measurements revealed clear Fabry-Pérot fringes the thickness cannot be evaluated at each measured $Z$ position. Clearly, the photonic crystal became thicker for positions $Z>3 \mathrm{~mm}$. For $Z<3 \mathrm{~mm}$ the thickness decrease, which means that part of the original opal is rinsed away during the second grow step. In addition, the number of layers of spheres that make up the crystal is calculated. Therefore, the sample thickness is divided by the distance $d_{111}$ between two layers of spheres. $d_{111}$ Follows from the face centered cubic closed packing of the spheres, and can be expressed as as a function of the sphere radius $r$ as:

$$
d_{111}=\frac{2}{3} \sqrt{6} r .
$$

From Figures 7.6 and 7.7 it is clear the additional vertical controlled drying step does not yield additional layers of spheres over the complete sample. In particular, for low $Z$ values the second vertical controlled drying step caused a decrease in the thickness. Also, reflectivity measurements revealed that the quality of the sample is not the same everywhere.

The alignment method described in this chapter was used to find the same 
domains in subsequent experiments. This study shows that the best area to mill defect on the opal is in the range $3 \leq Z \leq 3.5 \mathrm{~mm}$. At other locations the defect might be rinsed off or the sample may not be thick enough to show a high reflectivity. Hence, fabrication of material cavities is only useful, when the cavities are fabricated in the right region. The alignment method enabled us to find this particular region in the photonic crystal. Clearly, this knowledge makes the fabrication of nanometersized structures much more efficient.

\subsection{Conclusions}

In this chapter we have shown how to recover deterministically fabricated single nanostructures in various setups for non-contact experiments. We are able to probe these structures in a variety of ways without the need of alignment markers that may damage these structures. This enables the use of samples that are created solely by self-assembly. Structures smaller than the spatial resolution of the experimental setup can be probed as was shown with the use of the optical microscopes. The orientation of the fabricated nanostructure in the setup is revealed as well. Spatial maps are essential to find the nanostructures. In addition to these maps $(\mathrm{x}, \mathrm{y})$ coordinates relative to the corners of the sample are used to facilitate the alignment process. Furthermore, cross-correlation techniques were introduced as a possible approach to automate the alignment procedure. The experimental method was successfully used to align nanometer sized cavities on poorly conducting silica and polystyrene opal photonic crystals. Furthermore, the method was successfully applied to study opal layer growth processes. In general, the performance of noncontact measurements on one or several experimental setups can benefit from this approach. 



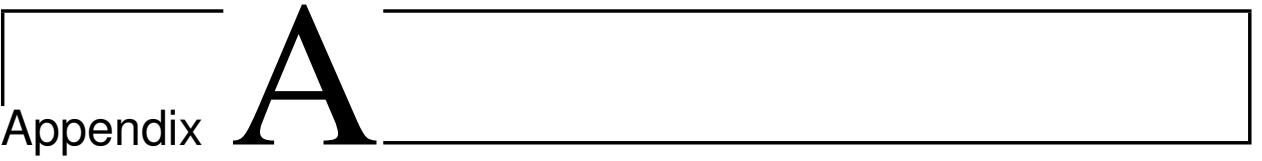

\section{List of hardware used in the near-infrared microscope}

Objectives, transparent at visible and near-infrared wavelengths

- Low magnification: 0.05, 0.07, and 0.12 NA

(MellesGriot: 04 OAS 002, 04 OAS 004, and 04 OAS 006 respectively)

- High magnification: CFI(LU)plan Epi SLWD 100X/0.70 (Nikon, experimentally determined to be transparent up to wavelengths $\lambda=2500 \mathrm{~nm}$ )

\section{Stages}

- Objective XYZ stages (PI: PIHera XYZ combination (stage), E-710.4CD (controller))

- Cryostat XY stages (Newport: XMS50 and XMS160 (stage), XPS-C2 (controller))

- Cryostat Z stage: lab-jack, origin unknown

- For lenses: manual stages with micrometer control (Thorlabs: e.g., PT1/M)

\section{Light sources}

- Fiber lamp: halogen cold light sources (Schott: KL2500 LCD)

- HeNe laser: continuous wave, $\lambda=633$ nm (Thorlabs: HRP050)

- Nd:YAG laser: continuous wave, $\lambda=1064 \mathrm{~nm}$ (Casix: LDC-1500)

- Diode laser: pulsed, repetition rate in steps till $40 \mathrm{MHz}, \lambda \approx 690 \mathrm{~nm}, 2 \mathrm{~mW}$ output (Picoquant: LDH-C-690 (laser head), and PDL 800-B (controller))

- Cougar laser: pulsed, including pulse-picker, operated at $\lambda=532 \mathrm{~nm}$ (TimeBandwidth: Cougar)

- Supercontinuum white light source $450<\lambda<2000$ nm (Fianium: SC450-2) 


\section{Spectrometer}

- Spectrograph (PI/Acton: sp2558)

turret with one gold mirror and two gratings: 1) $900 \mathrm{~g} / \mathrm{mm}$ Holographic, 2) $85 \mathrm{~g} / \mathrm{mm}$, blazed for $1.35 \mu \mathrm{m}$

- Imaging fiber adapter (PI/Acton: FC-446-030-SMA)

- Fiber optic bundle (PI/Acton: LG-456-020-3)

- InGaAs diode array (PI/Acton: OMA-V: 1024/LN)

\section{Detectors}

- Thermoelectric cooled near infrared photomultiplier module (Hamamatsu: H10330-75), used in combination with a wideband preamplifier module (Picoquant: PAM 102) and an adjustable attenuator (Agilent: 8494A)

- Spectrometer detector (PI/Acton: OMA-V: 1024/LN)

- Gray scale firewire camera (Allied Vision Technologies: DolphinF-145B), used in combination with a PCI-Express card (Belkin: FireWire 3-port PCI-Express card)

- Power meter, two amplified silicon detectors (Thorlabs: PDA36A)

- Fast near-infrared diode detector (Perkin Elmer: C30617 InGaAs pin photodiode based, see Appendix C for details)

- Hand held power meter (Ophir: PD300-3W (head), and Orion PD (display))

- Hand held infrared viewer (Laser Support Services: ELOP-SM-3R1700)

- Avalanche photodiodes: not yet implemented connected to setup using a 50/50 optical fiber multiplexer, or a regular 50/50 non-polarizing beam splitter.

\section{Cryostat}

Not yet implemented but commercially available, e.g., Microstat HiRes microscope cryostat (Oxford Instruments), or ST-500-LN microscope cryostat (Janis), or the RC102-CFM-HiRes-NOMOVE Microscopy Cryostat (Cryo Industries)

\section{Other}

- Timing module (Picoquant: PicoHarp 300 counting module)

- Enhanced aluminum and dielectric mirrors (1") in mounts with 100 tpi screws (Thorlabs)

- Aluminum coated parabolic mirrors: $90^{\circ}$ off-axis (Edmund optics)

- Filters: long-pass filters (Thorlabs: KG850 and FEL1100)

- Pinholes and dielectric (cold) mirror (Edmund optics)

- Pellicle beam splitters and achromat lenses (Thorlabs)

- Optical single mode $1064 \mathrm{~nm}$ and $532 \mathrm{~nm}$ fibers with pigtail connector/lens mounted (Oz optics) 
- Optical table 1.2 x 2.4 m, (TMC: 783-655-12R (CleanTop II series table top), and $14-42 \mathrm{H}-35$ (gimbal piston isolators, with casters))

- Breadboard (TMC: cleantop II, 78-113-12R)

- Rack mountable 13 slot PCI expansion rack (Magma: P13R1.5)

- Rack mountable connector accessory for DAQ devices (National Instruments: BNC-2090A) 

Appendix $\bigcirc$

\section{Derivation of exciton creation probability}

In Chapter 3 the role of multiple excitons in the study of quantum dots in photonic crystals is discussed. This appendix contains the derivation that yields an expression for the number of excitons created during a single excitation pulse.

Suppose the chance to create an exciton is independent of the number of excitons, existing in the quantum dot. This means that the quantum dots' absorption cross section $C_{\text {abs }}$ for exciton creation is constant. Furthermore, it is assumed that the excitation pulse duration is much shorter than the exciton decay time. Hence, only the number of photons in the excitation pulse is important and the pulse shape is unimportant. As a result, the chance $P$ for a single pulse to create a certain number of excitons $n_{x}$, is given by the binomial distribution [258]

$$
P\left(n_{x}\right)=\frac{N !}{n_{x} !\left(N-n_{x}\right) !} p^{n_{x}}(1-p)^{N-n_{x}}
$$

where $N$ is the number of photons in the excitation pulse, and $p$ is the chance for a single photon to create an exciton. In practice $N \rightarrow \infty$ and $p \rightarrow 0$, such that $N p \ll N$. Therefore, the binomial distribution can conveniently be approximated by the Poisson distribution, which gives

$$
P\left(n_{x}\right)=\frac{(N p)^{n_{x}} e^{-N p}}{n_{x} !}
$$

The mean number of excitons $\left\langle\mathrm{n}_{\mathrm{x}}\right\rangle$, created by a single pulse, is equal to the first moment of this distribution:

$$
\left\langle\mathrm{n}_{\mathrm{x}}\right\rangle=N p .
$$

The standard deviation $\sigma$ corresponding to this distribution is equal to the square 
root of the distributions' second moment:

$$
\sigma_{\left\langle\mathrm{n}_{\mathrm{x}}\right\rangle}=\sqrt{N p}
$$

The number of photons in the excitation pulse is simply given by the pulse energy $E$, divided by the energy per photon $\epsilon$, i.e.,

$$
N=\frac{E}{\epsilon}, \quad \text { with } \epsilon=\frac{h c}{\lambda_{0}} .
$$

Here $h$ is Planck's constant, $c$ is the speed of light, and $\lambda_{0}$ is the wavelength of the excitation light, in vacuum.

The remaining calculation to find an expression for $p$ consists of two steps. First, the probability distribution to find the photon at a certain position needs to be resolved. Secondly, the convolution between the probability distribution and the quantum dot's absorption cross-section is needed.

Consider the photon to be part of a collimated Gaussian beam $\left(\mathrm{TEM}_{00}\right)$. Hence, the average distribution of the photon in a plane perpendicular to the optical axis, is given by [75]

$$
\Phi(r)=\frac{2}{\pi w^{2}} e^{-2 r^{2} / w^{2}},
$$

where $r$ is the distance to the optical axis, and $w$ is the beam waist, i.e., the radius of the $\frac{1}{e^{2}}$ irradiance contour. If a collimated Gaussian beam is focused by a lens, the focal point waist radius $w_{0}$, the focal length $f$ of the lens, the Gaussian waist at the lens $w(f)$, and the wavelength $\lambda=\frac{\lambda_{0}}{n}$ are approximately related by

$$
w_{0} w(f) \approx \frac{f \lambda}{\pi}
$$

With use of the $99 \%$ criterium it is possible to relate $w_{0}$ to the wavelength of the excitation (in vacuum) $\lambda_{0}$, and the numerical aperture NA of the lens, which results in $[75]$

$$
w_{0} \approx \frac{\lambda_{0}}{2 \mathrm{NA}} .
$$

Combining Equations B.8 and B.6 yields the average photon distribution in the focal plane,

$$
\begin{aligned}
\Phi(r) & =\frac{2}{\pi w_{0}^{2}} e^{-2 r^{2} / w_{0}^{2}} \\
& \approx \frac{8 \mathrm{NA}^{2}}{\pi \lambda_{0}^{2}} e^{-8\left(r \mathrm{NA} / \lambda_{0}\right)^{2}} .
\end{aligned}
$$

Suppose a quantum dot is placed centrally in the focus of the laser beam. The chance $p$ for a single photon to hit the quantum dot and create an exciton can now be calculated by integrating Equation B.10 over the absorption cross-section $C_{\text {abs }}(\epsilon)$ 
of the quantum dot. This results in

$$
p=\int_{0}^{\sqrt{\frac{C_{\mathrm{abs}}(\epsilon)}{\pi}}} 2 \pi r \Phi(r) \mathrm{d} r .
$$

In practice the effective optical radius of a quantum $\operatorname{dot}\left(\sqrt{\frac{C_{\mathrm{abs}}(\epsilon)}{\pi}}\right)$ is very small compared to $w_{0}$ [259]. As a consequence, $\Phi(r)$ is effectively constant over the $r$-range used in the integral of Equation B.11. Therefore, Equation B.11 can be rewritten by replacing the integral over $r$ term by the absorption cross section multiplied by $\Phi(0)$. With use of Equation B.10, Equation B.11 simplifies to:

$$
p \approx C_{\mathrm{abs}}(\epsilon) \frac{8 \mathrm{NA}^{2}}{\pi \lambda_{0}^{2}}
$$

Finally, Equations B.12 and B.5 are substituted into Equation B.3. This results in the following expression for the mean number of excitons created by a single excitation pulse,

$$
\left\langle\mathrm{n}_{\mathrm{x}}\right\rangle=\frac{E}{\epsilon} \cdot \frac{8 C_{\mathrm{abs}}(\epsilon) \mathrm{NA}^{2}}{\pi \lambda_{0}^{2}} .
$$

This simple expression serves as an important tool during our experiments. We are now able to find sound experimental conditions, such that multiple-exciton generation can be ruled out. These experimental conditions are the topic of Section 3.3.2. 



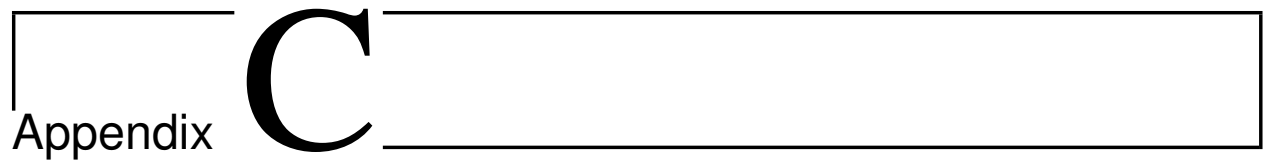

\section{Fast infrared diode}

This appendix contains background information on the detector used to determine the instrument response function in Section 5.2. We used a Perkin Elmer C30617 InGaAs pin photodiode to detect the $1064 \mathrm{~nm}$ pulses from our excitation laser (Time-Bandwidth, Cougar). The detector is designed such that the output is at $0 \mathrm{~V}$ when no light impinges on the detector. Infrared light pulses that impinge on the detector cause the output to go to negative voltages. Figure C.1 shows the electrical circuit to connect the photodiode. The elements are of the surface mount device type and the wiring is as short as feasible to assure the fastest system

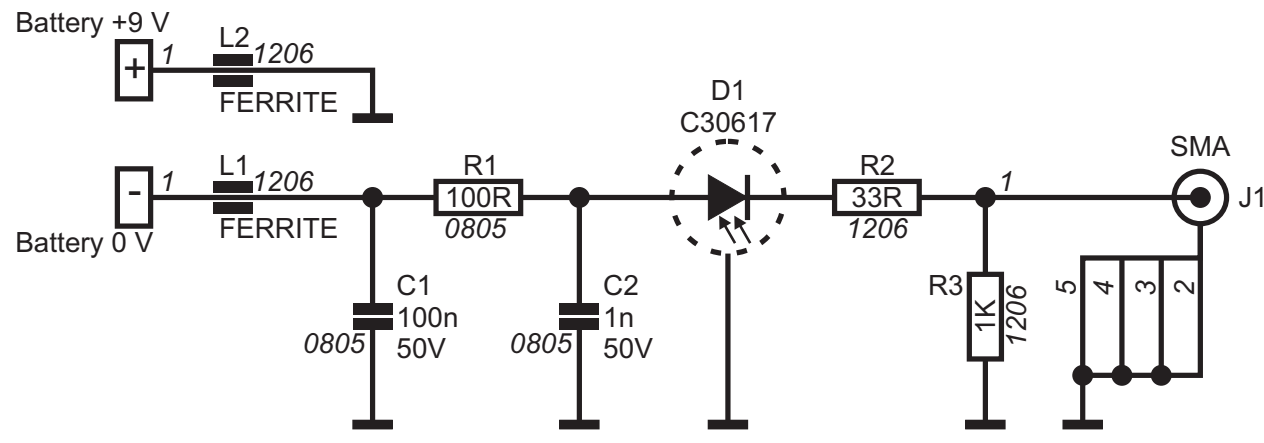

L1, L2: BCCOMPONENTS MLS1206-4S4-900

D1: PERKIN ELMER C30617 InGaAs PIN PHOTODIODE

Figure C.1: Electrical wiring scheme of our infrared detector, used to measure the instrument response function of our time correlated single photon counting setup. Slanted numbers beside the elements correspond either to measures of these surface mount devices or to an elements' pin number. (Design in collaboration with D. Verheijde, D. van Oosten, and A. Hartsuiker.) 

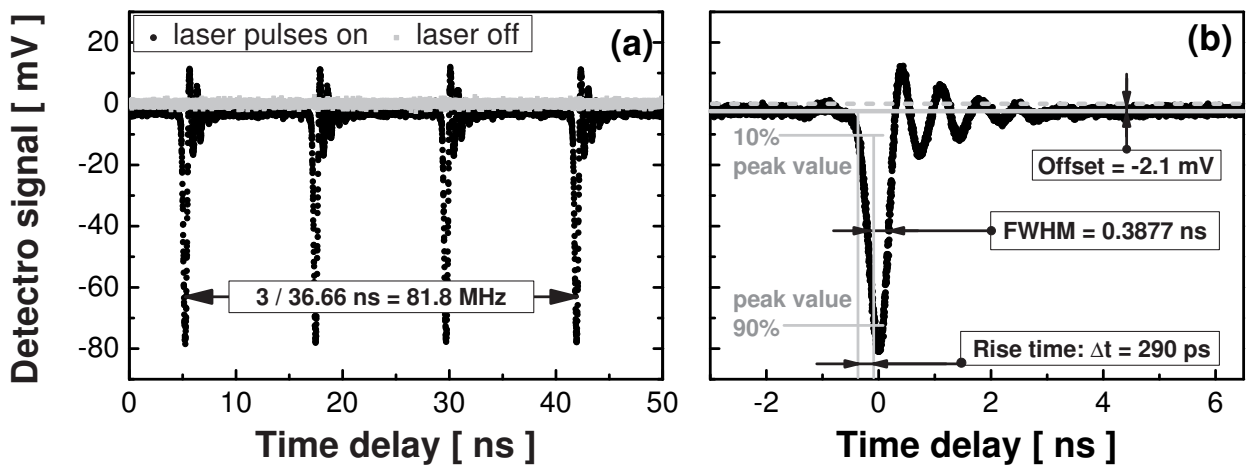

Figure C.2: Measured detector response to $1064 \mathrm{~nm}$ laser pulses of $11 \mathrm{ps}$ each. (a) Four subsequent pulses. The time difference between the pulses shows the expected repetition rate of $81.8 \mathrm{MHz}$. The small baseline offset form $0 \mathrm{mV}$ is caused by this high repetition rate. (b) Zoom in on single pulse. The detector response shows a fast voltage drop with a rise time of 290 ps. Subsequently, the output voltage decreases rapidly to its baseline value. Although ringing effects are observed, they are no limitation.

response possible. Furthermore, the power supply is stabilized with ferrite coils and a capacitor.

The detector response to the $1064 \mathrm{~nm}$ laser pulses of $11 \mathrm{ps}$ is measured with a fast digital oscilloscope (5Gs/s, 1GHz, Yokogawa, DLG/40L). Figure C.2.(a) shows a train of four subsequent pulses. Clearly the pulses show approximately the same amplitudes and shapes. Figure C.2.(b) contains a zoom in on a single pulse response. We observe a small baseline offset of $-2.1 \mathrm{mV}$, caused by the high repetition rate. The 10 to $90 \%$ rise time of the response is measured to be $290 \mathrm{ps}$ and the full width at half maximum is $388 \mathrm{ps}$. Some ringing is also observed, but because of its low amplitude it is not of any concern for our application, because it is well below the voltage discriminator level used in the counting processor module. 
Appendix

\section{Supplementary information}

\section{D.1 Titania inverse opal infiltration with PbSe quan- tum dots}

As mentioned in Section 4.2.2 two different infiltration techniques were used. The first approach was successfully used in Reference [30]. Therefore, butanol was added to the quantum dot in hexane suspension to make the suspension hydrophilic. The ratio between hexane and butanol was $1 \mathrm{v} / \mathrm{v}$. This approach leads to a clustering of the quantum dots in the suspension. These clusters were not instantaneously visible when butanol was added to the quantum dots in hexane suspension. However, photonic crystals that are infiltrated with this suspension turn from white into black.

Figure D.1.(a) shows a cross section of a non-photonic titania inverse opal, after infiltration with the PbSe quantum dots in hexane-butanol suspension. The center of the crystal still looks white, but at the sides the structure turned black. This black coloring is due to quantum dot clusters that infiltrate the crystal. Figure D.1.(b) shows the surface of a photonic crystal after infiltration of PbSe quantum dots in hexane. This sample does not show any black areas caused by quantum dots clusters. Therefore the second infiltration approach is used for further measurements.

Angle-resolved emission experiments also confirmed that the unsuccessfully infiltrated samples behave more like surface emitters (not shown). The different infiltration results are already clearly visible in single emission spectra. Figure D.2 shows the emission spectra measured from a non-photonic sample. Both spectra are measured from the same sample and scaled to the used excitation power. The gray dash-dotted curve shows the expected emission spectrum, and was taken from an area that looks white. The black line shows the spectrum, measured at a dark area. Clearly the latter is red shifted with respect to the former. Reabsorption of light causes a suppression of the emission intensity at the blue side in combination 

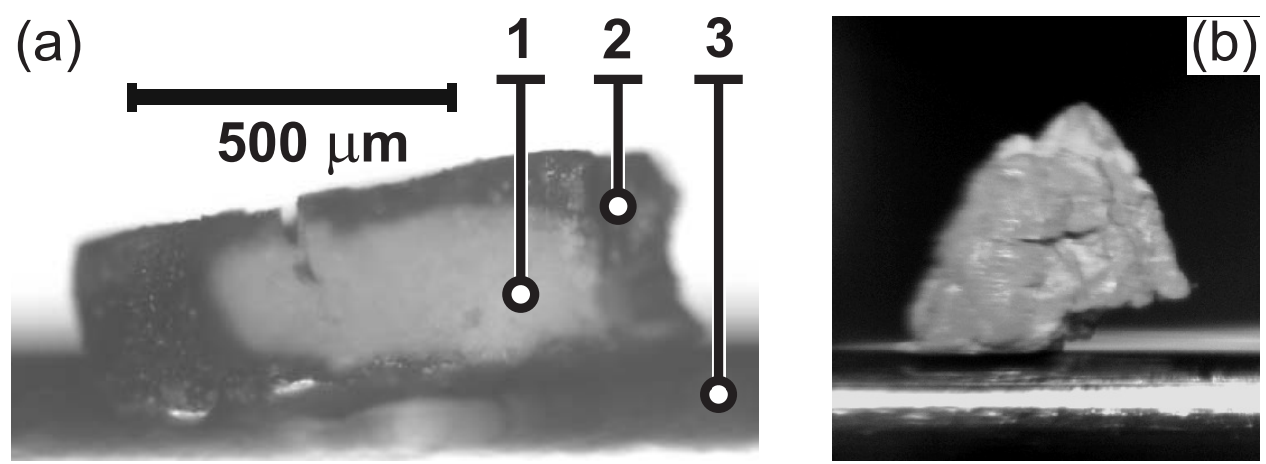

Figure D.1: (a) Bright field microscope image of unsuccessful infiltration of a titania inverse opal photonic crystal with PbSe quantum dots. The quantum dot suspension used contained both hexane and butanol $(50 / 50 \mathrm{v} / \mathrm{v})$. The image shows the cross section of the sample. (1) Indicates the white volume of the photonic crystals' inside. (2) Shows the crystals' surface layer that became dark due to the infiltrated PbSe quantum dot clusters, and (3) is the needle pin to which the sample was mounted. (b) Shows a grayscale microscope image of a successfully infiltrated sample, illuminated from the side. The only black area on the structure is a shadow effect. There is no sign of absorption due to quantum dot clusters.

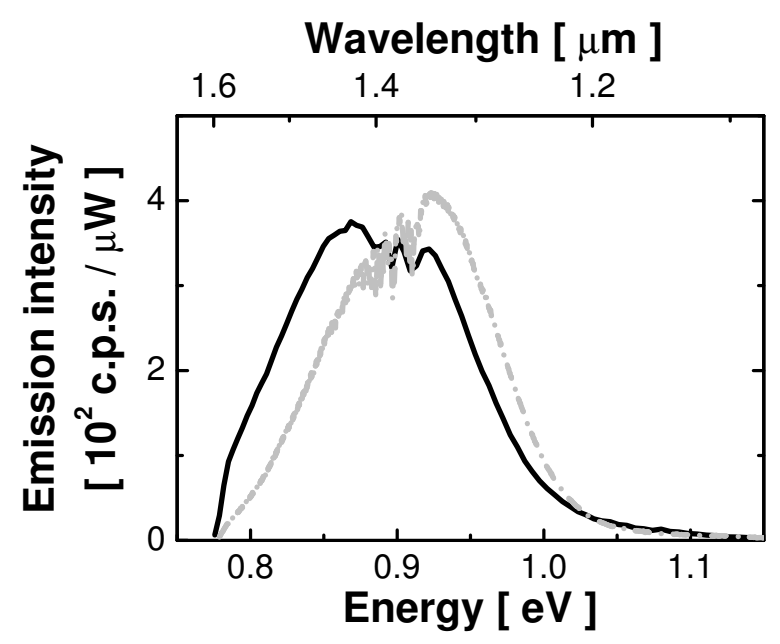

Figure D.2: Measured emission spectra from a non-photonic titania inverse opal. Dash-dotted line shows expected spectral shape, whereas the solid line shows reabsorption. Due to this reabsorption, the emission intensity of the latter is suppressed at the blue side and enhanced at the red side of the spectrum. 


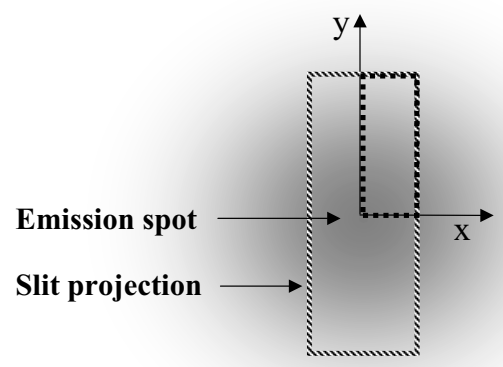

Figure D.3: Real-space cartoon of the projection of the spectrometer slit (striped rectangle) on the sample surface overlapped with the emission spot (in grey). One quarter of the slit projection (small dashed rectangle) has one of the corners in the emission-spot center. The width of the projection (along $x$ ) increases at larger detection angles $\Theta_{\mathrm{E}}$.

with an emission intensity enhancement at the red side.

\section{D.2 Correction for angle dependent emission col- lection efficiency}

This section is copied from Reference [14] for convenience of the reader.

The aim of this appendix is to explain the difference between the calculated escape distribution and the measured raw intensity illustrated in Fig. 4.9. This difference appears because the width of the projection of the collection optics (the spectrometer slit) on the sample increases with angle $\Theta_{\mathrm{E}}$. The spectrometer slit is imaged via objective lens $L$ onto the surface of the sample (Fig. 4.5). The only emission collected emanates from the region confined by the slit projection on the sample surface, see Fig. D.3. This means that the spectrometer collects light from a larger region on the surface at larger detection angles, and that the measured angle-dependent intensity should be corrected for the detection efficiency of the set-up. The detection efficiency is modeled as a ratio $D\left(\mu_{e}\right)$ of the intensity $B\left(\mu_{e}\right)$ collected from the surface region within the slit projection (Fig. D.3) at detection angle $\Theta_{\mathrm{E}}=\cos ^{-1}\left(\mu_{e}\right)$ to the intensity $B\left(\mu_{e}=1\right)$ collected at normal angle:

$$
D\left(\mu_{e}\right)=\frac{B\left(\mu_{e}\right)}{B\left(\mu_{e}=1\right)}, \quad B\left(\mu_{e}\right)=\int_{0}^{x\left(\mu_{e}\right)} \int_{0}^{y_{0}} I(x, y) d x d y
$$

We take into account that the integration runs over a quarter of the slit, as $x\left(\mu_{e}\right)=$ 
$x_{0} / \mu_{e}$ is the half-width of the slit projection, $x_{0}$ and $y_{0}$ are the half-width and the half-height of the slit projection at $\mu_{e}=\cos \left(\Theta_{\mathrm{E}}\right)=1$, respectively. Typical values of $x_{0}$ and $y_{0}$ in the experiments are $50 \mu \mathrm{m}$ and $250 \mu \mathrm{m}$. It is assumed that the intensity of diffuse light on the sample surface around the pump beam varies phenomenologically as $I(r) \propto \ell^{2} /\left(\ell^{2}+r^{2}\right)$, where $r^{2}=x^{2}+y^{2}$ is the distance from the axis of the pump beam along the sample surface, and $\ell$ is the transport mean free path of light in the sample.

\section{D.3 Parameters and results for first and second moment calculation}

This section contains supplementary information for Figure 5.13. Table D.1 contains the parameter sets used to calculate the average decay rates $\langle\gamma\rangle_{\lim }$ and variances $\sigma_{\lim }^{2}$ over a limited decay rate range $\gamma \in\left[\gamma_{\min }=1 / 900 \mathrm{~ns}, \gamma_{\max }=1 / 2 \mathrm{~ns}\right]$. Also, the mean and standard deviation of the results are calculated. A standard deviation of only $3 \%$ of the mean values was derived, both for the average decay rate and the variance of the distributions.

\begin{tabular}{|c|c|c|c|c|c|}
\hline \multicolumn{4}{|c|}{ Parameters } & \multicolumn{2}{|c|}{ Results } \\
\hline Set & width & {$\left[\begin{array}{c}\gamma_{\mathbf{m f}} \\
{\left[\mathbf{n s}^{-1}\right]}\end{array}\right.$} & {$\left[\begin{array}{c}\text { scale } \\
{[\text { counts s ] }}\end{array}\right.$} & {$\left[\begin{array}{c}\langle\gamma\rangle_{\lim } \\
{\left[\mathbf{n s}^{-1}\right]}\end{array}\right.$} & {$\left[\mathbf{n s}^{-2}\right]$} \\
\hline 1 & 3.4040 & $5.6460 \mathrm{E}-4$ & 521250 & 0.12025 & 0.01643 \\
\hline 2 & 3.3780 & $5.9715 \mathrm{E}-4$ & 514450 & 0.11984 & 0.01637 \\
\hline 3 & 3.3200 & $6.4900 \mathrm{E}-4$ & 509750 & 0.11776 & 0.01610 \\
\hline 4 & 3.2715 & $7.0210 \mathrm{E}-4$ & 504350 & 0.11625 & 0.01589 \\
\hline 5 & 3.2300 & $7.4790 \mathrm{E}-4$ & 500250 & 0.11483 & 0.01570 \\
\hline 6 & 3.1955 & $7.9370 \mathrm{E}-4$ & 495250 & 0.11383 & 0.01556 \\
\hline 7 & 3.1435 & $8.5580 \mathrm{E}-4$ & 490800 & 0.11190 & 0.01529 \\
\hline \multirow[t]{2}{*}{8} & 3.1070 & $9.0000 \mathrm{E}-4$ & 488400 & 0.11044 & 0.01509 \\
\hline & & & mean / sd & $0.116 / 0.004$ & $0.0158 / 5 \mathrm{E}-4$ \\
\hline
\end{tabular}

Table D.1: Overview of parameter values used to calculate the average decay rate $\langle\gamma\rangle_{\lim }$ and variance $\sigma_{\lim }^{2}$ shown in Figure 5.13. These combinations of parameters are approximately equally spaced on a line inside the volume enclosed by the iso-

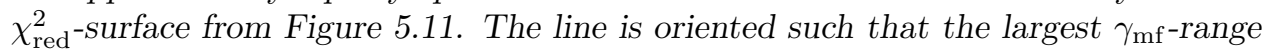
is probed. 


\section{Samenvatting}

\section{Beheersen van licht}

Lichtbronnen worden overal om ons heen gebruikt. De bekendste lichtbron is natuurlijk de zon, die het leven op aarde mogelijk maakt. Daarnaast kent iedereen de gloeilamp, de tl-buis en LED-lampjes. Het licht dat afstraalt van deze bronnen is afkomstig van atomen, of een verzameling atomen. Een atoom is als het ware een elementaire lichtbron. Uit de opbouw van een atoom kunnen we verklaren wat de oorsprong is van het licht dat door atomen wordt uitgezonden. Het linkerdeel van Figuur S.1 toont een model van een atoom, welke bestaat uit een kern, waar

\section{atoom model}

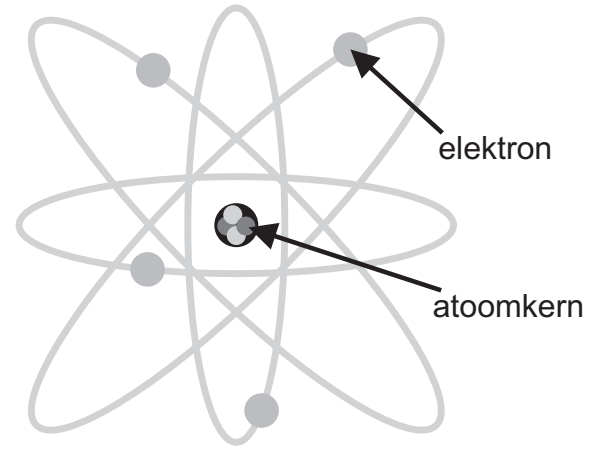

energie schema

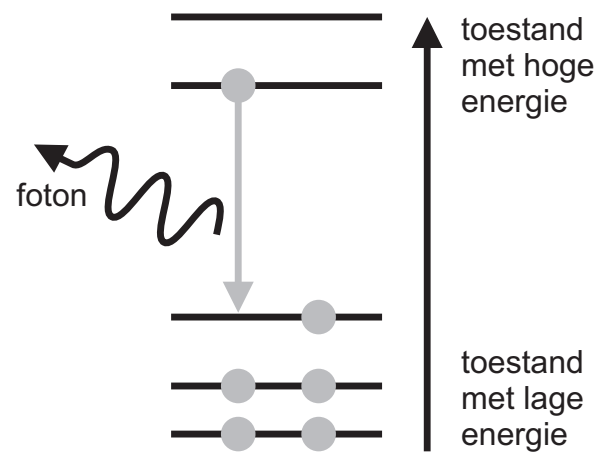

Figuur S.1: Links: Zéér schematische weergave van een atoommodel, bestaande uit een atoomkern, waar elektronen omheen bewegen. Rechts: schematische weergave van energie toestanden voor elektronenen in een atoom. Een elektron valt terug naar een lagere energie toestand onder gelijktijdige uitzending van een lichtpakketje: een foton. 
elektronen omheen bewegen. De energie van een elektron in een atoom kan slechts een beperkt aantal scherp bepaalde waarden hebben en is zogezegd gekwantiseerd. Er wordt daarom ook wel gesproken van de energie toestanden waarin een elektron zich kan bevinden binnen het atoom. Er geldt dat zich slechts twee elektronen in dezelfde energie toestand kunnen bevinden en dat in de natuur gestreefd wordt naar de laagst mogelijke energie toestand: de grondtoestand. Een atoom bevindt zich in een aangeslagen toestand wanneer lagere energie toestanden beschikbaar zijn voor elektronen. Een atoom kan weer terugkeren naar zijn grondtoestand wanneer de elektronen overgaan tot het bezetten van enkel en alleen de toestanden met de laagst mogelijke energie. Dit proces staat schematisch weergegeven aan de rechterkant van Figuur S.1. Een elektron in een toestand met een hoge energie valt terug naar een lagere energie toestand (aangegeven door de verticale grijze pijl). Het energieverschil tussen de aangeslagen toestand waarin een elektron zich oorspronkelijk bevindt en de lagere energie toestand waar het elektron toe overgaat wordt door het atoom uitgezonden als een energiepakketje, dat foton wordt genoemd.

Licht, zoals wij dat met onze ogen waarnemen, bestaat uit fotonen. Het woord foton is afgeleid van het Griekse woord voor licht $=\varphi$ o $\tau o \varsigma($ photos $)$. De basis van dit begrip van licht, als opgebouwd uit losse pakketjes met een goedgedefinieerde energie, is te danken aan de wetenschappers Max Planck en Albert Einstein. Naast dit deeltjeskarakter heeft licht ook een golfkarakter. Van golven weten we dat ze elkaar kunnen versterken of verzwakken; dit wordt interferentie genoemd. Interferentie verschijnselen zijn eenvoudig te zien met watergolven in een (zwem)bad. Wanneer beide handen op het wateroppervlak worden gehouden (de handpalm in het water, de rug van de hand in de lucht) en op enige afstand van elkaar gelijktijdig en snel op en neer worden bewogen ontstaan golven op het wateroppervlak. Midden tussen de handen zal het water sterk op en neer deinen, terwijl die deining op een andere plek veel zwakker is. Wanneer daarentegen de handen in tegengestelde richting worden bewogen is de deining midden tussen de handen relatief klein. De golflengte (de afstand tussen twee toppen) wordt bepaald door de frequentie waarmee de handen op en neer worden bewogen. Dit is analoog aan het golfkarakter van fotonen, waar de golflengte wordt bepaald door de energie van het foton. De kleur van het licht is gegeven door de golflengte. Het verschil in energie tussen de verschillende elektron toestanden in een atoom bepaalt dus welke kleuren licht het atoom uit kan zenden.

In tegenstelling tot wat misschien wordt verwacht, wordt de kleur van het uitgezonden licht en de snelheid waarmee een aangeslagen atoom terugkeert naar zijn grondtoestand niet alleen bepaald door het atoom zelf. Ook de omgeving waarin het atoom is geplaatst speelt een bepalende rol. Niet alleen moet er in het atoom een lagere energietoestand beschikbaar zijn voor het elektron, ook moet er buiten het atoom een toestand voorhanden zijn waarin het foton kan bestaan. De hoeveelheid aan mogelijke foton toestanden wordt gevat in een grootheid die de LDOS wordt genoemd. Vertaald uit het Engels staat de afkorting LDOS voor: locale dichtheid van foton toestanden. Deze LDOS geeft aan hoe makkelijk een lichtbron energie kan uitzenden in de vorm van fotonen. Kwantum mechanisch wordt met de LDOS 
berekend hoe efficient een lichtbron op een bepaalde plaats en met een bepaalde orientatie fotonen met een bepaalde kleur kan uitzenden in de beschikbare foton toestanden. Hoe lager de LDOS, hoe moeilijker het voor de lichtbron is een foton uit te zenden en hoe langer het gemiddeld duurt voordat licht wordt uitgezonden. Aan de andere kant geldt: hoe hoger de LDOS, hoe sneller de lichtbron gemiddeld een foton uitzendt. De tijd dat een atoom in een aangeslagen toestand blijft ligt dus niet vast. Bij het bestuderen van emissieprocessen is het daarom van belang om naast de energie van de fotonen ook het tijdsgedrag van de foton-emissie te kunnen meten. Hiertoe worden zogenaamde tijdsopgeloste metingen verricht.

Omdat de omgeving de lichtbron beïnvloed is het mogelijk om eigenschappen van lichtbronnen te beheersen, door middel van de omgeving. Om meer beheersing over lichtbronnen te krijgen bestuderen wij in dit proefschrift hoe lichtbronnen met hun omgeving wisselwerken. De fundamentele processen die een rol spelen bij deze wisselwerking zijn wetenschappelijk erg interessant. Meer begrip van deze processen maakt het mogelijk om kunstmatige omgevingen te ontwerpen waarin lichtbronnen een vooraf bepaald, gewenst gedrag vertonen. De mogelijkheid om lichtbronnen te beheersen is bijzonder interessant voor toepassingen. Deze beheersing maakt het bijvoorbeeld mogelijk lichtbronnen efficiënter en daarmee (energie)zuiniger te maken en de energie omzetting van zonlicht naar elektriciteit te verbeteren in zonnecellen. Ook zijn lichtbronnen essentieel voor telecommunicatie waar licht wordt gebruikt als signaal om informatie over te dragen, zoals bijvoorbeeld tussen een afstandsbediening en een televisie toestel, of in communicatie netwerken gemaakt van glasvezel. In de kwantum computers van de toekomst kunnen lichtbronnen dienst doen als de bits in een geheugenmodule of als signaalbron als alternatief voor de momenteel gebruikelijke elektrische signalen.

\section{Fotonische kristallen en lichtbronnen}

In 1987 was er een grote doorbraak in de natuurkunde. In dat jaar werd voorspeld dat dielectrische fotonische kristallen een fotonische bandkloof kunnnen hebben, waarin geldt: $\operatorname{LDOS}=0$. Een fotonisch kristal is dus een hele extreme omgeving voor een lichtbron. Doordat de LDOS gelijk wordt aan 0 kunnen fotonen niet propageren door de structuur. Dientengevolge kan een lichtbron geen fotonen uitzenden en daarom blijft deze als het ware gevangen in een aangeslagen toestand.

Een fotonisch kristal is een periodieke ruimtelijke structuur welke typisch is opgebouwd uit verschillende doorzichtige (transparante) materialen, bijvoorbeeld glas en lucht. Figuur S.2 toont schematisch hoe zo'n structuur er uit ziet. Het linker plaatje toont een gelaagde structuur, die bijvoorbeeld zorgt voor het typische kleurenspel van paarlemoer. In het middelste plaatje staat een structuur met een periodieke structuur in twee richtingen en in het rechter plaatje staat een structuur die in drie richtingen periodiek is. Deze laatste vinden we in de natuur bijvoorbeeld in opalen, die vaak in sieraden worden gebruikt en bestaan uit een mooie periodieke stapeling van kleine glasachtige deeltjes. 


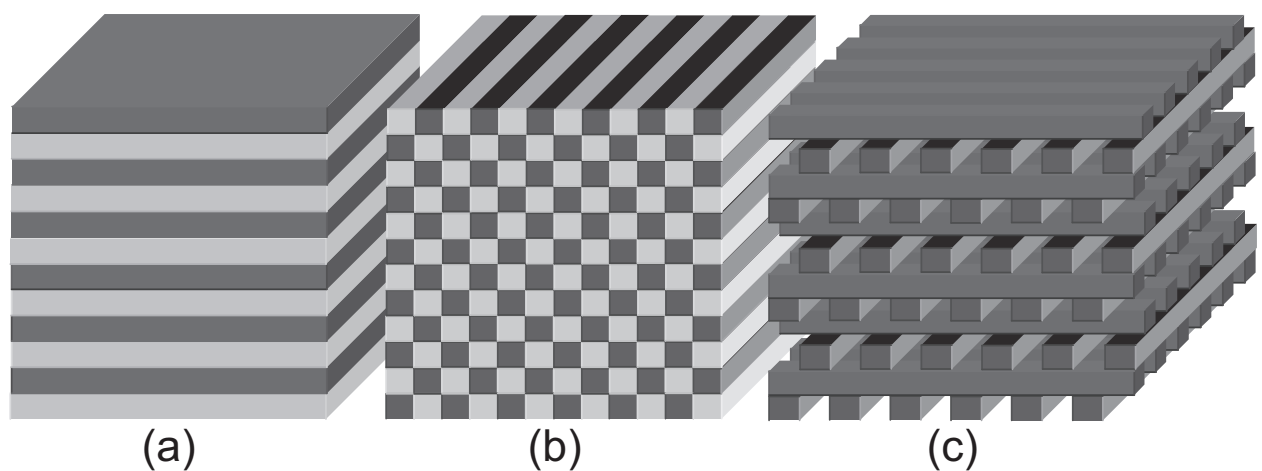

Figuur S.2: Schematische weergave van fotonische structuren. (a) In één richting periodieke structuur, (b) fotonisch kristal met periodiciteit in twee richtingen, (c) fotonisch kristal welke periodiek is in drie richtingen. De donkere en lichte kleuren stellen de verschillende materialen voor waaruit de structuren zijn opgebouwd. Dit plaatje is afkomstig uit het proefschrift van Ivan Nikolaev [14].

Wanneer we naar zo'n fotonisch kristal als een opaal kijken verschijnen mooie kleuren (opalescentie), die veranderen wanneer het opaal gedraaid wordt (zie ook de foto op de voorkant van dit proefschrift). Deze kleuren ontstaan door interferentie effecten. Wanneer de halve golflengte overeen komt met de afstand die in het kristal moet worden afgelegd voordat de structuur zich herhaalt (de periode), zal het licht dat gereflecteerd wordt door de materiaalovergangen in de structuur constructief interfereren. Dit betekent dat het licht teruggekaatst wordt door het kristal. Andere golflengten die niet passen op de structuur van het kristal kunnen wel doorgelaten worden door het kristal.

Door een slimme keuze van de kristal structuur en de gebruikte materialen is het mogelijk een kristal te maken met de genoemde bandkloof. Hiervoor zijn doorzichtige materialen nodig waarin het licht zich met een zo groot mogelijk verschillende snelheid voortbeweegt. In lucht heeft het licht praktisch de hoogst en in silicium praktisch de laagst mogelijke snelheid. Daarom willen we hier graag de kristallen van maken. Silicium wordt eveneens heel veel gebruikt in electronica, wat ons werk mogelijk voor een groot vakgebied relevant maakt. Bovendien ontstaan er mogelijkheden voor intrigerende combinaties van electronica en nanofotonica.

In plaats van licht van buiten op zulke kristallen te sturen plaatsen wij lichtbronnen in het kristal. Omdat silicium transparant is wanneer de golflengte van het licht groter is dan 0.0011 millimeter zoeken we lichtbronnen die in dat regime hun licht uitzenden. Wij gebruiken daarom clusters van atomen, die ook wel kwantum punten (Engels: quantum dots) worden genoemd. Kwantumpunten kunnen worden beschouwd als artificiële atomen in de vaste stof fysica. De genoemde golflengtes maken deel uit van het gebied dat bekend staat onder de verzamelnaam nabijinfrarode golflengten. Tevens komt dit golflengte gebied overeen met de kleuren 
licht die gebruikt worden voor telecommunicatie doeleinden, wat ons werk mogelijk relevant maakt voor een breed bereik aan toepassingen.

Met behulp van fotonische kristallen willen wij dus de LDOS beheersen, om zo de eigenschappen van onze lichtbronnen te veranderen. Trilholtes en puntdefecten in fotonische kristallen kunnen de LDOS sterk beïnvloeden en zijn daarom ook zeer relevant voor ons onderzoek. Het is mogelijk kunstmatig trilholtes te maken en de emissie van licht door bronnen die geplaatst zijn in de trilholte te bestuderen. Hiervoor is wel een geavanceerde microscoop opstelling noodzakelijk. Momenteel wordt in onze onderzoeksgroep gewerkt aan de fabricage van defecten in fotonische kristallen met een bandkloof. Deze defecten zorgen ervoor dat locaal de LDOS niet meer nul is en dat een lichtbron dus toch zijn licht uit kan zenden. Echter, het licht kan dan nog steeds niet het kristal verlaten en blijft bij de lichtbron in de buurt, waar het uiteindelijk weer kan worden opgenomen door de lichtbron. In dit geval beïnvloedt de lichtbron dus direct zijn omgeving, waar de lichtbron wel of geen foton voelt, en daarmee zijn eigen eigenschappen. Als gevolg kunnen de energie toestanden in de lichtbron niet meer worden beschreven zonder te letten op de omgeving van de lichtbron. Hier begint het interessante regime van sterke koppeling, waar de lichtbron zogezegd sterk gekoppeld is aan zijn omgeving. In dit regime willen we in de toekomst metingen doen.

\section{Inhoud van dit proefschrift}

- Hoofdstuk 1 bevat een algemene introductie in dit vakgebied. Hierin staan onze motivaties opgesomd en worden onze doelen duidelijk gemaakt. Daarnaast wordt het verband tussen de verschillende hoofdstukken uitgelegd.

- Hoofdstuk 2 beschrijft de experimentele opstelling die wij gebouwd hebben voor onze nieuwe experimenten bij nabij-infrarode en zichtbare golflengten. $\mathrm{Na}$ een discussie over de vereisten en de verschillende mogelijkheden is gekozen voor het zelf bouwen van een geavanceerde microscoop opstelling. Met deze opstelling zijn we in staat emissie van lichtbronnen in fotonische kristallen te bestuderen, waarbij bijzondere aandacht is besteed aan nauwkeurige, ruimtelijke positioneringsmogelijkheden. Daarnaast kunnen de structuren worden afgekoeld naar hele lage temperaturen (tot wel $-265^{\circ} \mathrm{C}$ ). De opstelling is computer gestuurd, zodat we in staat zijn automatisch emissie eigenschappen over een monster in kaart te brengen. Het ontwerp van de opstelling is zodanig dat alle onderdelen los van elkaar gebruikt kan worden, wat deze opstelling bijzonder flexibel maakt.

- Hoofdstuk 3 bevat karakterisatie metingen aan onze lichtbronnen, kwantum punten gemaakt van lood $(\mathrm{Pb})$ en seleen $(\mathrm{Se})$. Hier vinden we de werkzame licht absorptiedoorsnede van de punten, welke rond de $10^{-16}$ tot $10^{-15} \mathrm{~cm}^{2}$ ligt ( $10^{-16}$ betekent 1 gedeeld door een 1 met 16 nullen). Daarnaast zijn de absorptie en emissie sterktes bepaald en de resultaten zijn gebruikt om te verklaren waarom, tegen de verwachting in, bij lood-selenide de grotere 
kwantum punt lichtbronnen sneller licht uitzenden dan de kleinere punten. In dit hoofdstuk concluderen we ook dat de individuele kwantum punten een veel groter bereik aan kleuren kan uitzenden dan verwacht.

- Hoofdstuk 4 beschouwt de eerste metingen aan de fotonische kristallen met de lichbronnen: onze monsters. Omdat de gewenste silicium kristallen nog niet waren voltooid hebben we onze kwantum punt lichtbronnen in zogenaamde luchtbol kristallen gestopt (zie Figuur 1.3 ter illustratie). Deze kristallen zijn gemaakt van titaandioxide (titania): een mineraal dat bijvoorbeeld zorgt voor de witte kleur in papier, verf en tandpasta . Allereerst worden de kristallen gekarakteriseerd met behulp van optische microscopie en reflectiemetingen. Vervolgens zijn metingen gedaan waarbij gemeten wordt hoeveel licht van de kwantum punten onder een bepaalde hoek het monster verlaat. We laten zien dat de resultaten sterk bepaald zijn door verstrooiing en interferentie van het licht in het fotonische kristal. Een ontsnappingsmodel is toegepast om de verdeling van het uitgezonden licht over de mogelijke uittree hoeken kwantitatief te beschrijven.

- Hoofdstuk 5 bevat de experimentele details die in ogenschouw moeten worden genomen wanneer we willen meten hoe lang een aangeslagen lichtbron er over doet om naar zijn grondtoestand terug te keren onder uitzending van licht. Met name voor metingen bij nabij-infrarode licht golflengten komt het te meten signaal nauwelijks boven de ruis van de detector uit. We laten zien hoe we in deze situatie, door de juiste keuze van experimentele instellingen, toch de interessante signalen kunnen detecteren.

- Hoofdstuk 6 gebruikt de kennis uit het voorgaande hoofdstuk om de emissie snelheid van kwantum punt lichtbronnen in fotonische kristallen te meten. Om de LDOS aan te passen veranderen we de periode van het kristal en we laten zien dat dit gepaard gaat met een verandering in de emissie snelheid. In dit hoofdstuk demonstreren we als eersten ter wereld dat we met fotonische kristallen deze zogenaamde kwantum lichtbronnen kunnen beheersen bij nabijinfrarode golflengtes. We hebben een emissie-versnelling van $29 \%$ gemeten en een vertraging van $51 \%$. Dit is een belangrijk succes dat ons een grote stap dichter brengt bij de experimenten met fotonische bandgap materialen. Deze demonstratie betekent eveneens dat de door ons gebouwde opstelling voldoet aan de experimentele eisen.

- Hoofdstuk 7 bevat de uitleg van de techniek die we hebben ontwikkeld om in staat te zijn in verschillende opstellingen dezelfde kleine structuren terug te vinden. In plaats van kunstmatige markeerpunten aan te brengen laten we zien dat intrinsieke structuren op monsters voldoende zijn om als referentiekader te dienen. Met voorbeelden uit de praktijk wordt de methode geïllustreerd. Deze techniek zullen we later nodig hebben om onze trilholtes in fotonische kristallen terug te vinden. 


\section{Dankwoord}

Een proefschrift is niet compleet zonder dankwoord, omdat de beschreven resultaten mede mogelijk zijn gemaakt door de ondersteuning van vele collegae, familie en vrienden. Allereerst bedank ik Willem Vos, mijn promotor. Willem, onder jouw vleugels ben ik enorm gegroeid als onderzoeker. Daarvoor ben ik je heel dankbaar. Ik weet zeker dat je jouw invloed herkent in mijn manier van werken en dank je nogmaals voor je voortdurende steun en begeleiding, welke onontbeerlijk bleken voor het behalen van onze resultaten. Ik heb veel lering getrokken uit jouw drive naar fysisch begrip. Ook Ad Lagendijk bedank ik voor zijn begeleiding en zijn wijze lessen op het gebied van eerlijke en heldere wetenschapscommunicatie. Ad, ik bewonder jouw vaardigheid om door te dringen tot de kern van een (fysisch) vraagstuk. Ook is mijn aanstelling bij COPS aan jou te danken, waarvoor ik zeer erkentelijk ben.

Voor experimentele begeleiding wil ik Ivan Nikolaev bedanken. Dankzij hem kreeg ik snel de fijne kneepjes van ons vak onder de knie. Ivan, jouw hulp bij de fabricage van onze monsters en het uitlijnen van optische meetopstellingen bleek cruciaal en hebben mij veel tijdwinst opgeleverd. Ook Rob van Loon heeft hierbij een rol gespeeld. Dankzij hen kon ik snel onze verschillende meetopstellingen gebruiken. Femius Koenderink bedank ik voor zijn begeleiding bij de interpretatie van mijn meetresultaten alsook voor de vele discussies over de escape functie. Femius, ik waardeer je constructieve bijdrage aan het onderzoek enorm en prijs me gelukkig dat je zo goed bereikbaar was tijdens mijn promotieonderzoek.

Veel dank aan Léon Woldering, Willem Tjerkstra, Charles Uju en Steven Kettelarij die hard hebben gewerkt aan de fabricage van de fotonische kristallen die nodig waren voor het onderzoek. Steven, ik ben heel blij dat jij de fabricage af hebt weten te ronden voor je aan je nieuwe baan begon, zodat de data voor Hoofdstukken 4 en 6 konden worden gemeten. Bedankt voor je hulp bij het vinden van een geschikte methode om onze quantum dots in de kristallen te stoppen. Léon ben ik erg dankbaar voor zijn begeleiding in het chemische lab en zijn persoonlijke betrokkenheid. Hannie van den Broek bedank ik voor de snelle hulp bij het vinden 
en opsturen van het laatste fotonische kristal dat ik nodig had om de metingen compleet te maken. Hugo Schlatter (UvA) bedank ik voor het beschikbaar stellen van zijn handschoenenkast, waarmee ik mijn experimenten kon beginnen.

I am very grateful to Florian Kulzer for sharing his knowledge on single-molecule experiments. The design of our new setup (Chapter 2) has benefitted strongly from our discussions. In addition I thank Takashi Kuroda, Bennet Goldberg, and Richard Younger for sharing their expertise on microscope cryostat scanning experiments. Your input convinced me to apply this technique to our measurements, which was a crucial step for our setup design. Voor algemene technische ondersteuning bedank ik op de eerste plaats Cock Harteveld, die mij vanaf de begintijd in Enschede begeleidde. Cock, ik ben erg blij dat jij me met je goede raad terzijde bleef staan, ook toen ik met een deel van de groep naar Amsterdam verhuisde. Daarnaast waardeer ik het zeer dat jij je al die jaren persoonlijk betrokken hebt getoond.

Veel dank aan alle mensen van de ondersteunende diensten binnen AMOLF en COPS. In het bijzonder Sjoerd Wouda, Marco Konijnenburg, Duncan Verheijde, Iliya Cerjak en Wim Brouwer voor hun ondersteuning bij de opbouw van onze nieuwe meetopstelling. Verder Iwert Mijnders, Rob Kemper, Hinco Schoenmaker, Ernst Prins en Ilja Stavenuiter voor de algemene ondersteuning in onze experimenteerruimte. Tatjana van der Ben en Johan Stiel voor hun hulp bij de inkopen en het administreren van de onderzoeksbudgetten. Voor computer en netwerk ondersteuning dank ik de ICT groep en Bas Benschop in het bijzonder voor al je hulp bij de laptop problemen. Voor administratieve begeleiding bedank ik Karen Munnink, Justine Knowles, Piet Kistemaker en Raymond Oudeboon. Marjo Wijnands en Hillie de Jong-Kuijt bedank ik voor hun betrokkenheid en persoonlijke interesse.

Dries van Oosten, Alex Hartsuiker en Duncan Verheijde dank ik voor de hulp bij het ontwerpen van een snel elektronisch circuit voor het karakteriseren van onze meetopstelling. Robert van der Meer, Tom Savels, Bernard Kaas, Alex Hartsuiker, Allard Mosk, Ad Lagendijk en Willem Vos bedank ik voor hun inzet bij ons sideproject: het opzetten van een gezamenlijke literatuurdatabase. Het was voor mij een hele leerzame ervaring en het resultaat mag er zijn: dit is het eerste proefschrift dat met behulp van die database is gemaakt.

Zinnige discussies over optica, fotonische kristallen, lichtverstrooiing en experimentele opstellingen had ik met velen: Allard Mosk, Daniël Vanmaekelbergh, Tijmen Euser, Tom Savels, Karen van der Molen, Philip Harding, Bernard Kaas, Paolo Scalia, Ivo Vellekoop, Boris Bret, Peter Lodahl, Christian Blum, Georgios Ctistis, Edwin van der Pol, Niels Zijlstra, mijn afstudeerstudent Oscar Bok, Pedro de Vries, Vitali Prosentsov, Sanli Faez, Otto Muskens, Patrick Johnson, Alex Hartsuiker, Steven Kettelarij, Merel Leistikow en Ramy El-Dardiry. Allen hartelijke dank. Ik dank de groepsleiders Albert Polman, Kobus Kuipers, Femius Koenderink, Jaime Gómez Rivas, Ad Lagendijk en Willem Vos voor het stimuleren van contacten, discussies en samenwerkingsverbanden tussen de verschillende onderzoeksgroepen van het Center for Nanophotonics. Ik vond het erg bijzonder om deel uit te maken van dit door jullie opgezette centrum. 
Mijn (oud) kamergenoten Tania Oudemans, Silke Diedenhofen en Bernard Kaas, Ivan Nikolaev, Merel Leistikow en Ramy El-Dardiry verdienen bijzondere aandacht. Met hen en Willem Vos deelde ik de ups en downs van het onderzoek en als er wat te lachen viel, dan was het met jullie. Bedankt voor jullie hulp en steun. Hoewel we nooit kamergenoten waren valt dezelfde dank ten deel aan Alex Hartsuiker. Alex, heel erg bedankt voor de vele discussies over ons onderzoek en de openhartige gesprekken die we in de loop der jaren hebben gevoerd.

Buiten de naaste collegae bedank ik graag mijn paranimfen Dirk Bekke en Sijmen Gerritsen voor hun morele en praktische ondersteuning voorafgaand aan, tijdens en na afloop van de promotieplechtigheid. Ook mijn vrienden van de Bracque en hun aanhang, Coffee Corner alsook mijn vrienden en vriendinnen uit Oldenzaal en omstreken wil ik graag bedanken voor hun interesse in de voortgang van mijn onderzoek en mijn welbevinden; bedankt voor jullie steun. Mijn familie, ouders, zussen en zwagers ben ik heel dankbaar voor hun morele steun, luisterend oor en voor hun betrokkenheid bij het welzijn van ons gezin. Mijn schoonouders bedank ik voor hun vele bezoeken aan Zwolle. Jullie praktische inzet en hulp waren een grote steun voor Wendy en mij.

Een promotieonderzoek trekt een zware wissel op iemands privé leven. Gelukkig is er een geweldige vrouw die mij tijdens het onderzoek bijstond met haar voortdurende zorg en liefde. Lieve Wendy, alle ogen waren vaak op mij gericht, omdat ik het onderzoek deed. Daarom zijn er maar weinig mensen die beseffen hoezeer deze promotietijd een last op jouw schouders betekende. Mijn lange reistijd van en naar Amsterdam, de vele overuren en treinvertragingen betekenden enerzijds dat je er overdag en 's avonds vaak alleen voor stond bij de zorg voor onze kinderen en anderzijds dat er weinig ruimte was voor leuke dingen. Ik hoop dat we Gwendolyn en Waldemar binnenkort uit kunnen leggen dat papa met de trein / papa werken niet betekent dat papa bij de spoorwegen werkt. Wendy, mijn dank aan jou is onbeschrijfelijk. 


\section{Bibliography}

[1] E. Yablonovitch, Inhibited Spontaneous Emission in Solid-State Physics and Electronics, Phys. Rev. Lett. 58, 2059-2062 (1987).

[2] H.-G. Park, S.-H. Kim, S.-H. Kwon, Y.-G. Ju, J.-K. Yang, J.-H. Baek, S.-B. Kim, and Y.-H. Lee, Electrically Driven Single-Cell Photonic Crystal Laser, Science 305, 1444-1447 (2004).

[3] M. Grätzel, Photoelectrochemical cells, Nature 414, 338-344 (2001).

[4] I. Rodriguez, P. Atienzar, F. Ramiro-Manzano, F. Meseguer, A. Corma, and A. Garcia, Photonic crystals for applications in photoelectrochemical processes Photoelectrochemical solar cells with inverse opal topology, Photonics Nanostruct. 3, 148-154 (2005).

[5] M. A. Nielsen and I. L. Chuang, Quantum computation and quantum information, 2000 .

[6] E. M. Purcell, Spontaneous emission probabilities at radio frequencies, Phys. Rev. 69, 681 (1946).

[7] A. Lagendijk, Vibrational relaxation studied with light, in: Ultrashort Processes in Condensed Matter, Edited by W. E. Bron, Plenum, New York, 1993.

[8] E. Jaynes and F. Cummings, Comparison of Quantum and Semiclassical Radiation Theories with Application to the Beam Maser, Proc. IEEE 51, 89-109 (1963).

[9] L. Allen and J. H. Eberly, Optical resonances and two-level atoms, Dover Publications, Inc., New York, 1987.

[10] C. Cohen-Tannoudji, J. Dupont-Roc, and G. Grynberg, Atom-Photon Interactions, John Wiley \& Sons, Inc., New York, 1992.

[11] B. Shore and P. Knight, Topical review, The Jaynes-Cummings model, J. Mod. Optics 40, 1195-1238 (1993).

[12] E. Fermi, Quantum Theory of Radiation, Rev. Mod. Phys. 4, 87-132 (1932).

[13] R. Loudon, The quantum theory of light, Oxford University Press, 2nd edition, 1983.

[14] I. S. Nikolaev, Spontaneous-emission rates of quantum dots and dyes controlled with photonic crystals, $\mathrm{PhD}$ thesis, University of Twente, available online: http://www.photonicbandgaps.com, 2006.

[15] A. F. Koenderink, Emission and Transport of Light in Photonic Crystals, PhD thesis, University of Amsterdam, available online: http://www.photonicbandgaps.com, 2003.

[16] R. Sprik, B. A. van Tiggelen, and A. Lagendijk, Optical emission in periodic dielectrics, Europhys. Lett. 35, 265-270 (1996).

[17] J. M. Gérard, D. Barrier, J. Y. Marzin, and T. Rivera, Quantum boxes as active probes for photonic microstructures: The pillar microcavity case, Appl. Phys. Lett. 69, 449-451 (1996).

[18] M. Fujita, A. Sugitatsu, T. Uesugi, and S. Noda, Fabrication of Indium Phosphide Compound Photonic Crystal by Hydrogen Iodide/Xenon Inductively Coupled Plasma Etching, Jpn. J. Appl. Phys. 43, L1400-L1402 (2004). 
[19] L. A. Woldering, Fabrication of photonic crystals and nanocavities, $\mathrm{PhD}$ thesis, University of Twente, available online: http://www.photonicbandgaps.com, 2008.

[20] J. M. Gérard, B. Sermage, B. Gayral, B. Legrand, E. Costard, and V. ThierryMieg, Enhanced Spontaneous Emission by Quantum Boxes in a Monolithic Optical Microcavity, Phys. Rev. Lett. 81, 1110-1113 (1998).

[21] M. Bayer, T. L. Reinecke, F. Weidner, A. Larionov, A. McDonald, and A. Forchel, Inhibition and Enhancement of the Spontaneous Emission of Quantum Dots in Structured Microresonators, Phys. Rev. Lett. 86, 3168-3171 (2001).

[22] G. S. Solomon, M. Pelton, and Y. Yamamoto, Single-mode Spontaneous Emission from a Single Quantum Dot in a Three-Dimensional Microcavity, Phys. Rev. Lett. 86, 3903-3906 (2001).

[23] B. Gayral, J.-M. Gérard, B. Sermage, A. Lemaître, and C. Dupuis, Time-resolved probing of the Purcell effect for InAs quantum boxes in GaAs microdisks, Appl. Phys. Lett. 78, 2828-2830 (2001).

[24] D. Englund, D. Fattal, E. Waks, G. Solomon, B. Zhang, T. Nakaoka, Y. Arakawa, Y. Yamamoto, and J. Vučković, Controlling the Spontaneous Emission Rate of Single Quantum Dots in a Two-Dimensional Photonic Crystal, Phys. Rev. Lett. 95, 013904 (2005).

[25] A. Kress, F. Hofbauer, N. Reinelt, M. Kaniber, H. J. Krenner, R. Meyer, G. Böhm, and J. J. Finley, Manipulation of the spontaneous emission dynamics of quantum dots in two-dimensional photonic crystals, Phys. Rev. B 71, 241304 (2005).

[26] A. J. Bennett, D. J. P. Ellis, A. J. Shields, P. Atkinson, I. Farrer, and D. A. Ritchie, Observation of the Purcell effect in high-index-contrast micropillars, Appl. Phys. Lett. 90, 191911 (2007).

[27] L. Balet, M. Francardi, A. Gerardino, N. Chauvin, N. Le Thomas, R. Houdré, and A. Fiore, Enhanced spontaneous emission rate from single InAs quantum dots in a photonic crystal nanocavity at telecom wavelengths, Appl. Phys. Lett. 91, 123115 (2007).

[28] T. Suzuki and P. K. L. Yu, Emission power of an electric dipole in the photonic band structure of the fcc lattice, J. Opt. Soc. Am. B 12, 570-582 (1995).

[29] K. Busch and S. John, Photonic band gap formation in certain self-organizing systems, Phys. Rev. E 58, 3896-3908 (1998).

[30] P. Lodahl, A. F. van Driel, I. S. Nikolaev, A. Irman, K. Overgaag, D. Vanmaekelbergh, and W. L. Vos, Controlling the dynamics of spontaneous emission from quantum dots by photonic crystals, Nature 430, 654-657 (2004).

[31] M. Fujita, S. Takahashi, Y. Tanaka, T. Asano, and S. Noda, Simultaneous inhibition and redistribution of spontaneous light emission in photonic crystals, Science 308, 1296-1298 (2005).

[32] M. Fujita, S. Takahashi, T. Asano, Y. Tanaka, and S. Noda, Controlled spontaneousemission phenomena in semiconductor slabs with a two-dimensional photonic bandgap, J. Opt. A: Pure Appl. Opt. 8, s131-s138 (2006).

[33] I. S. Nikolaev, P. Lodahl, A. F. van Driel, A. F. Koenderink, and W. L. Vos, 
Strongly nonexponential time-resolved fluorescence of quantum-dot ensembles in three-dimensional photonic crystals, Phys. Rev. B 75, 115302 (2007).

[34] R. A. L. Vallée, K. Baert, B. Kolaric, M. van der Auweraer, and K. Clays, Nonexponential decay of spontaneous emission from an ensemble of molecules in photonic crystals, Phys. Rev. B 76, 045113 (2007).

[35] I. S. Nikolaev, P. Lodahl, and W. L. Vos, Fluorescence lifetime of emitters with broad homogeneous linewidths modified in opal photonic crystals, J. Phys. Chem. C 112, 7250-7254 (2008).

[36] B. Julsgaard, J. Johansen, S. Stobbe, T. Stolberg-Rohr, T. S ünner, M. Kamp, A. Forchel, and P. Lodahl, Decay dynamics of quantum dots influenced by the local density of optical states of two-dimensional photonic crystal membranes, Appl. Phys. Lett. 93, 094102 (2008).

[37] J. P. Reithmaier, S. Sek, A. Loffler, C. Hofmann, S. Kuhn, S. Reitzenstein, L. V. Keldysh, V. D. Kulakovskii, T. L. Reinecke, and A. Forchel, Strong coupling in a single quantum dot-semiconductor microcavity system, Nature 432, 197-200 (2004).

[38] T. Yoshie, A. Scherer, J. Hendrickson, G. Khitrova, H. M. Gibbs, G. Rupper, C. Ell, O. B. Shchekin, and D. G. Deppe, Vacuum Rabi splitting with a single quantum dot in a photonic crystal nanocavity, Nature 432, 200-203 (2004).

[39] E. Peter, P. Senellart, D. Martrou, A. Lemaître, J. Hours, J. M. Gérard, and J. Bloch, Exciton-photon strong-coupling regime for a single quantum dot embedded in a microcavity, Phys. Rev. Lett. 95, 067401 (2005).

[40] G. Khitrova, H. M. Gibbs, M. Kira, S. W. Koch, and A. Scherer, Vacuum Rabi splitting in semiconductors, Nature Phys. 2, 81-90 (2006).

[41] E. Peter, J. Bloch, D. Martrou, A. Lemaître, J. Hours, G. Patriarche, A. Cavanna, J. M. Gérard, S. Laurent, I. Robert-Philip, and P. Senellart, Cavity QED with a single QD inside an optical microcavity, Phys. Stat. Sol. B 243, 3879-3884 (2006).

[42] K. Hennessy, A. Badolato, M. Winger, D. Gerace, M. Atatüure, S. Gulde, S. Fält, E. L. Hu, and A. Imamoğlu, Quantum nature of a strongly coupled single quantum dotcavity system, Nature 445, 896-899 (2007).

[43] D. Englund, A. Faraon, I. Fushman, N. Stoltz, P. Petroff, and J. Vučković, Controlling cavity reflectivity with a single quantum dot, Nature 450, 857-861 (2007).

[44] S. Reitzenstein, C. Hofmann, A. Gorbunov, M. Strauss, S. H. Kwon, C. Schneider, A. Löffler, S. Höfling, M. Kamp, and A. Forchel, AlAs/GaAs micropillar cavities with quality factors exceeding 150.000, Appl. Phys. Lett. 90, 251109 (2007).

[45] K. Srinivasan and O. Painter, Linear and nonlinear optical spectroscopy of a strongly coupled microdiskquantum dot system, Nature 450, 862-865 (2007).

[46] G. Manzacca, G. Cincotti, and K. Hingerl, Ultrafast switching by controlling Rabi splitting, Appl. Phys. Lett. 91, 231920 (2007).

[47] M. Nomura, Y. Ota, N. Kumagai, S. Iwamoto, and Y. Arakawa, Large Vacuum Rabi Splitting in Single Self-Assembled Quantum Dot-Nanocavity System, Appl. Phys. Express 1, 072102 (2008).

[48] C. Kistner, T. Heindel, C. Schneider, A. Rahimi-Iman, S. Reitzenstein, S. Höfling, 
and A. Forchel, Demonstration of strong coupling via electro-optical tuning in highquality QD-micropillar systems, Opt. Express 16, 15006 (2008).

[49] E. d. Valle, F. P. Laussy, F. M. Souza, and I. A. Shelykh, Optical spectra of a quantum dot in a microcavity in the nonlinear regime, Phys. Rev. B 78, 085304 (2008).

[50] Y. Ota, M. Shirane, M. Nomura, N. Kumagai, S. Ishida, S. Iwamoto, S. Yorozu, and Y. Arakawa, Vacuum Rabi splitting with a single quantum dot embedded in a H1 photonic crystal nanocavity, Appl. Phys. Lett. 94, 033102 (2009).

[51] P. Kristensen, A. F. Koenderink, P. Lodahl, B. Tromborg, and J. Mørk, Fractional decay of quantum dots in real photonic crystals, Opt. Lett. 33, 1557-1559 (2008).

[52] A. Hartsuiker, B. H. Husken, M. D. Leistikow, J. M. Gérard, and W. L. Vos, Report on switching the decay rate of an emitter inside a cavity, 2008.

[53] P. R. Villeneuve, S. Fan, and J. D. Joannopoulos, Microcavities in photonic crystals: Mode symmetry, tunability, and coupling efficiency, Phys. Rev. B 54, 7837-7842 (1996).

[54] M. Okano, A. Chutinan, and S. Noda, Analysis and design of single-defect cavities in a three-dimensional photonic crystal, Phys. Rev. B 66, 165211 (2002).

[55] N. W. Ashcroft and N. D. Mermin, Solid State Physics, Brooks/Cole, 1976.

[56] W. L. Vos, R. Sprik, A. van Blaaderen, A. Imhof, A. Lagendijk, and G. H. Wegdam, Strong effects of photonic band structures on the diffraction of colloidal crystals, Phys. Rev. B 53, 16231-16235 (1996).

[57] M. Born and E. Wolf, Principles of Optics, Cambridge University Press, 4th edition, 1997.

[58] J. E. G. J. Wijnhoven, L. Bechger, and W. L. Vos, Fabrication and characterization of large macroporous photonic crystals in titania, Chem. Mater. 13, 4486-4494 (2001).

[59] R. Spry and D. Kosan, Theoretical Analysis of the Crystalline Colloidal Array Filter, Appl.Spectroscopy 40, 782 (1986).

[60] H. M. van Driel and W. L. Vos, Multiple Bragg wave coupling in photonic band-gap crystals, Phys. Rev. B 62, 9872-9875 (2000).

[61] K. M. Ho, C. T. Chan, C. M. Soukoulis, R. Biswas, and M. Sigalas, Photonic band gaps in three dimensions: New layer-by-layer periodic structures, Solid State Commun. 89, 413-416 (1994).

[62] R. Hillebrand, S. Senz, W. Hergert, and U. Gösele, Macroporous silicon-based threedimensional photonic crystal with a large complete band gap, J. Appl. Phys. 94, 2758-2760 (2003).

[63] M. Hermatschweiler, A. Ledermann, G. A. Ozin, M. Wegener, and G. von Freymann, Fabrication of Silicon Inverse Woodpile Photonic Crystals, Adv. Funct. Mat. 17, 2273-2277 (2007).

[64] A. Polman, Erbium implanted thin film photonic materials, J. Appl. Phys. 82, 39 (1997). 
[65] M. A. Kastner, Artificial atoms, Phys. Today 46, 24-31 (1993).

[66] M. Bayer, O. Stern, P. Hawrylak, S. Fafard, and A. Forchel, Hidden symmetries in the energy levels of excitonic 'artificial atoms', Nature 405, 923-926 (2000).

[67] F. W. Wise, Lead Salt Quantum Dots: the Limit of Strong Quantum Confinement, Acc. Chem. Res. 33, 773-780 (2000).

[68] B. L. Wehrenberg, C. Wang, and P. Guyot-Sionnest, Interband and Intraband Optical Studies of PbSe Colloidal Quantum Dots, J. Phys. Chem. B 106, 1063410640 (2002).

[69] M. Megens, Structure and fluorescence of photonic colloidal crystals, PhD thesis, University of Amsterdam, available online: http://www.photonicbandgaps.com, 1999.

[70] A. F. Koenderink, R. de Waele, J. C. Prangsma, and A. Polman, Experimental evidence for large dynamic effects on the plasmon dispersion of subwavelength metal nanoparticle waveguides, Phys. Rev. B 76, 201403 (2007).

[71] K. Yoshino, Y. Shimoda, Y. Kawagishi, K. Nakayama, and M. Ozaki, Temperature tuning of the stop band in transmission spectra of liquid-crystal infiltrated synthetic opal as tunable photonic crystal, Appl. Phys. Lett. 75, 932-934 (1999).

[72] B. Wild, R. Ferrini, R. Houdré, M. Mulot, S. Anand, and C. J. M. Smith, Temperature tuning of the optical properties of planar photonic crystal microcavities, Appl. Phys. Lett. 84, 846-848 (2004).

[73] B. Gralak, M. J. A. de Dood, G. Tayeb, S. Enoch, and D. Maystre, Theoretical study of photonic band gaps in woodpile crystals, Phys. Rev. E 67, 066601 (2003).

[74] T. Savels, Scattering lasers, PhD thesis, University of Twente, available online: http://www.randomlasers.com, 2007.

[75] A. E. Siegman, Lasers, University Science Books, Sausalito, California, 1986.

[76] A. Hartsuiker, P. J. Harding, Y.-R. Nowicki-Bringuier, J.-M. Gérard, and W. L. Vos, Kerr and free carrier ultrafast all-optical switching of GaAs/AlAs nanostructures near the three photon edge of GaAs, J. Appl. Phys. 104, 083105 (2008).

[77] E. Flück, Local interaction of light with periodic photonic structures, $\mathrm{PhD}$ thesis, University of Twente, available online: http://os.tnw.utwente.nl, 2003.

[78] M. Grundmann, J. Christen, N. N. Ledentsov, J. Böhrer, D. Bimberg, S. S. Ruvimov, P. Werner, U. Richter, U. Gösele, J. Heydenreich, V. M. Ustinov, A. Y. Egorov, A. E. Zhukov, and Kopev, Ultranarrow Luminescence Lines from Single Quantum Dots, Phys. Rev. Lett. 74, 4043-4046 (1995).

[79] S. A. Empedocles, D. J. Norris, and M. G. Bawendi, Photoluminescence Spectroscopy of Single CdSe Nanocrystallite Quantum Dots, Phys. Rev. Lett. 77, 38733876 (1996).

[80] T. Miyazawa, K. Takemoto, Y. Sakuma, S. Hirose, T. Usuki, N. Yokoyama, M. Takatsu, and Y. Arakawa, Single-Photon Generation in the 1.55- $\mu$ m OpticalFiber Band from an InAs/InP Quantum Dot, Jpn. J. Appl. Phys. 44, L620-L622 (2005).

[81] M. Megens, J. E. G. J. Wijnhoven, A. Lagendijk, and W. L. Vos, Fluorescence 
lifetimes and linewidths of dye in photonic crystals, Phys. Rev. A 59, 4727-4731 (1999).

[82] A. F. Koenderink, L. Bechger, H. P. Schriemer, A. Lagendijk, and W. L. Vos, Broadband Fivefold Reduction of Vacuum Fluctuations Probed by Dyes in Photonic Crystals, Phys. Rev. Lett. 88, 143903 (2002).

[83] M. J. de Dood, A. Polman, and J. G. Fleming, Modified spontaneous emission from erbium-doped photonic layer-by-layer crystals, Phys. Rev. B 67, 115106 (2003).

[84] M. Barth, A. Gruber, and F. Cichos, Spectral and angular redistribution of photoluminescence near a photonic stop band, Phys. Rev. B 72, 085129 (2005).

[85] C. Brunel, B. Lounis, P. Tamarat, and M. Orrit, Triggered Source of Single Photons based on Controlled Single Molecule Fluorescence, Phys. Rev. Lett. 83, 2722-2725 (1999).

[86] B. Lounis and M. Orrit, Single-photon sources, Rep. Prog. Phys. 68, 1129-1179 (2005).

[87] T. Kuroda, M. Abbarchi, T. Mano, K. Watanabe, M. Yamagiwa, K. Kuroda, K. Sakoda, G. Kido, N. Koguchi, C. Mastrandrea, L. Cavigli, M. Gurioli, Y. Ogawa, and F. Minami, Photon Correlation in GaAs Self-Assembled Quantum Dots, Appl. Phys. Express 1, 042001 (2008).

[88] J.-M. Segura, A. Renn, and B. Hecht, A sample-scanning confocal optical microscope for cryogenic operation, Rev. Sci. Instrum. 71, 1706-1711 (2000).

[89] M. Orrit and J. Bernard, Single pentacene molecules detected by fluorescence excitation in a p-terphenyl crystal, Phys. Rev. Lett. 65, 2716-2719 (1990).

[90] M. Vácha, H. Yokoyama, T. Tokizaki, M. Furuki, and T. Tani, Laser scanning microscope for low temperature single molecule and microscale spectroscopy based on gradient index optics, Rev. Sci. Instrum. 70, 2041-2045 (1999).

[91] L. Novotny and B. Hecht, Principles of Nano-Optics, Cambridge University Press, United Kingdom, 2006.

[92] L. A. Woldering, R. W. Tjerkstra, H. V. Jansen, I. D. Setija, and W. L. Vos, Periodic arrays of deep nanopores made in silicon with reactive ion etching and deep UV lithography, Nanotechnology 19, 145304: 1-11 (2008).

[93] A. I. Ekimov and A. A. Onushchenko, Quantum size effect in three-dimensional microscopic semiconductor crystals, JETP Lett. 34, 345-349 (1981).

[94] Al. L. Efros and A. L. Efros, Interband absorption of light in a semiconductor sphere, Sov. Phys. Semicond. 16, 772-775 (1982).

[95] L. E. Brus, A simple model for the ionization potential, electron affinity, and aqueous redox potentials of small semiconductor crystallites, J. Chem. Phys. 79, 5566-5571 (1983).

[96] A. I. Ekimov, A. L. Efros, and A. A. Onushchenko, Quantum size effect in semiconductor microcrystals, Solid State Commun. 56, 921-924 (1985).

[97] A. P. Alivisatos, Semiconductor Clusters, Nanocrystals, and Quantum Dots, Science 271, 933-937 (1996). 
[98] G. Allan and C. Delerue, Confinement effects in PbSe quantum wells and nanocrystals, Phys. Rev. B 70, 245321 (2004).

[99] C. Burda, X. Chen, R. Narayanan, and M. A. El-Sayed, Chemistry and Properties of Nanocrystals of Different Shapes, Chem. Rev. 105, 1025-1102 (2005).

[100] P. Guyot-Sionnest, Colloidal quantum dots, Recent advances in quantum dot physics / Nouveaux développements dans la physique des boîtes quantiques, C. R. Physique 9, 777-787 (2008).

[101] R. D. Schaller, M. A. Petruska, and V. I. Klimov, Tunable Near-Infrared Optical Gain and Amplified Spontaneous Emission Using PbSe Nanocrystals, J. Phys. Chem. B 107, 13265-13768 (2003).

[102] A. Franceschetti, Structural and electronic properties of PbSe nanocrystals from first principles, Phys. Rev. B 78, 075418 (2008).

[103] M. V. Artemyev, A. I. Bibik, L. I. Gurinovich, S. V. Gaponenko, and U. Woggon, Evolution from individual to collective electron states in a dense quantum dot ensemble, Phys. Rev. B 60, 1504-1506 (1999).

[104] C. B. Murray, C. R. Kagan, and M. G. Bawendi, Synthesis and characterization of monodisperse nanocrystals and close-packed nanocrystal assemblies, Annu. Rev. Mater. Sci. 30, 545-610 (2000).

[105] F. X. Redl, K.-S. Cho, C. B. Murray, and S. O'Brien, Three-dimensional binary superlattices of magnetic nanocrystals and semiconductor quantum dots, Nature 423, 968-971 (2003).

[106] D. Yu, C. Wang, B. L. Wehrenberg, and P. Guyot-Sionnest, Variable Range Hopping Conduction in Semiconductor Nanocrystal Solids, Phys. Rev. Lett. 92, 216802 (2004).

[107] H. E. Romero and M. Drndic, Coulomb Blockade and Hopping Conduction in PbSe Quantum Dots, Phys. Rev. Lett. 95, 156801 (2005).

[108] E. V. Shevchenko, D. V. Talapin, N. A. Kotov, S. OBrien, and C. B. Murray, Structural diversity in binary nanoparticle superlattices, Nature 439, 55-59 (2006).

[109] P. Liljeroth, K. Overgaag, A. Urbieta, B. Grandidier, S. G. Hickey, and D. Vanmaekelbergh, Variable Orbital Coupling in a Two-Dimensional Quantum-Dot Solid Probed on a Local Scale, Phys. Rev. Lett. 97, 096803 (2006).

[110] P. M. Petroff, A. Lorke, and A. Imamoglu, Epitaxially self-assembled quantum dots, Phys. Today 54, 46-52 (2001).

[111] E. H. Sargent, Infrared Quantum Dots, Adv. Mater. 17, 515-522 (2005).

[112] U. Woggon, Single semiconductor nanocrystals: Physics and applications, J. Appl. Phys. 101, 081727 (2007).

[113] K. K. Zhuravlev, J. M. Pietryga, R. K. Sander, and R. D. Schaller, Optical properties of PbSe nanocrystal quantum dots under pressure, Appl. Phys. Lett. 90, 043110 (2007).

[114] A. J. Shields, Semiconductor quantum light sources, Nat. Phot. 1, 215-223 (2007).

[115] E. Holder, N. Tesslerb, and A. L. Rogach, Hybrid nanocomposite materials with 
organic and inorganic components for opto-electronic devices, J. Mater. Chem. 18, 1064-1078 (2008).

[116] A. P. Alivisatos, Less is More in Medicine, Sci. Am. 285, 66-73 (2001).

[117] C. Seydel, Quantum Dots Get Wet, Science 300, 80-81 (2003).

[118] J. K. Jaiswal and S. M. Simon, Potentials and pitfalls of fluorescent, Trends Cell Biol. 14, 497-504 (2004).

[119] X. Michalet, F. F. Pinaud, L. A. Bentolila, A. M. Wu, S. S. Gambhir, and S. Weiss, Quantum Dots for Live Cells, in Vivo Imaging, and Diagnostics, Science 307, 538 544 (2005).

[120] R. Weissleder and P. M. J., Imaging in the era of molecular oncology, Nature 452, 580-589 (2008).

[121] V. L. Colvin, M. C. Schlamp, and A. P. Alivisatos, Light-emitting diodes made from cadmium selenide nanocrystals and a semiconducting polymer, Nature 370, 354-357 (1994).

[122] B. O. Dabbousi, M. G. Bawendi, O. Onitsuka, and M. F. Rubner, Electroluminescence from CdSe quantum-dot/polymer composites, Appl. Phys. Lett. 66, 1316-1318 (1995).

[123] F. Hatami, V. Lordi, J. S. Harris, H. Kostial, and W. T. Masselink, Red lightemitting diodes based on InP/GaP quantum dots, J. Appl. Phys. 97, 096106 (2005).

[124] V. I. Klimov, A. A. Mikhailovsky, S. Xu, A. Malko, and M. G. Bawendi, Optical Gain and Stimulated Emission in Nanocrystal Quantum Dots, Science 290, 314-317 (2000).

[125] K. Inoue, H. Sasaki, K. Ishida, Y. Sugimoto, and K. Asakawa, InAs quantum-dot laser utilizing GaAs photonic-crystal line-defect waveguide, Opt. Express 12, 5502 5509 (2004).

[126] V. Sukhovatkin, S. Musikhin, and I. a. Gorelikov, Room-temperature amplified spontaneous emission at $1300 \mathrm{~nm}$ in solution-processed $\mathrm{PbS}$ quantum-dot films, Opt. Lett. 30, 171-173 (2005).

[127] N. Yamamoto, K. Akahane, A. U. S. Gozu, and N. Ohtani, 1.55- $\mu \mathrm{m}$-Waveband Emissions from Sb-Based Quantum-Dot Vertical-Cavity Surface-Emitting Laser Structures Fabricated on GaAs Substrate, Jpn. J. Appl. Phys. 45, 3423-3426 (2006).

[128] S. Hoogland, V. Sukhovatkin, I. Howard, S. Cauchi, L. Levina, and E. H. Sargent, A solution-processed $1.53 \mu \mathrm{m}$ quantum dot laser with temperature-invariant emission wavelength, Opt. Express 14, 3273-3281 (2006).

[129] E. Lifshitz, M. Brumer, A. Kigel, A. Sashchiuk, M. Bashouti, M. Sirota, E. Galun, Z. Burshtein, A. Q. Le Quang, I. Ledoux-Rak, and J. Zyss, Air-Stable PbSe/PbS and $\mathrm{PbSe} / \mathrm{PbSe}_{x} \mathrm{~S}_{1-x}$ Core-Shell Nanocrystal Quantum Dots and Their Applications, J. Phys. Chem. B 110, 25356-25365 (2006).

[130] J. L. Machol, F. W. Wise, R. Patel, and D. B. Tanner, Optical studies of IV-VI quantum dots, Physica A 207, 427-434 (1994).

[131] M. Kuno, J. K. Lee, B. O. Dabbousi, F. V. Mikulec, and M. G. Bawendi, The band edge luminescence of surface modified CdSe nanocrystallites: Probing the luminesc- 
ing state, J. Chem. Phys. 106, 9869-9882 (1997).

[132] J. M. An, A. Franceschetti, and A. Zunger, The Excitonic Exchange Splitting and Radiative Lifetime in PbSe Quantum Dots, Nano Lett. 7, 2129-2135 (2007).

[133] A. Olkhovets, R.-C. Hsu, A. Lipovskii, and F. W. Wise, Size-Dependent Temperature Variation of the Energy Gap in Lead-Salt Quantum Dots, Phys. Rev. Lett. 81, 35393542 (1998).

[134] A. Lipovskii, E. Kolobkova, V. Petrikov, I. Kang, A. Olkhovets, T. Krauss, M. Thomas, J. Silcox, F. Wise, Q. Shen, and S. Kycia, Synthesis and characterization of PbSe quantum dots in phosphate glass, Appl. Phys. Lett. 71, 3406-3408 (1997).

[135] C. B. Murray, S. Sun, W. Gaschler, H. Doyle, T. A. Betley, and C. R. Kagan, Colloidal synthesis of nanocrystals and nanocrystal superlattices, IBM J. Res. Dev. 45, 47-56 (2001).

[136] H. Du, C. Chen, R. Krishnan, T. D. Krauss, J. M. Harbold, F. W. Wise, M. G. Thomas, and J. Silcox, Optical Properties of Colloidal PbSe Nanocrystals, Nano Lett. 2, 1321-1324 (2002).

[137] W. W. Yu, J. C. Falkner, B. S. Shih, and V. L. Colvin, Preparation and Characterization of Monodisperse PbSe Semiconductor Nanocrystals in a Noncoordinating Solvent, Chem. Mater. 16, 3318-3322 (2004).

[138] R. J. Ellingson, M. C. Beard, J. C. Johnson, P. Yu, O. I. Micic, A. J. Nozik, A. Shabaev, and A. L. Efros, Highly Efficient Multiple Exciton Generation in Colloidal PbSe and PbS Quantum Dots, Nano Lett. 5, 865-871 (2005).

[139] R. D. Schaller, M. Sykora, S. Jeong, and V. I. Klimov, High-Efficiency Carrier Multiplication and Ultrafast Charge Separation in Semiconductor Nanocrystals Studied via Time-Resolved Photoluminescence, J. Phys. Chem. B 110, 25332-25338 (2006).

[140] R. D. Schaller, M. Sykora, J. M. Pietryga, and V. I. Klimov, Seven Excitons at a Cost of One: Redefining the Limits for Conversion Efficiency of Photons into Charge Carriers, Nano Lett. 6, 424-429 (2006).

[141] A. Franceschetti, J. M. An, and A. A. Zunger, Impact Ionization Can Explain Carrier Multiplication in PbSe Quantum Dots, Nano Lett. 6, 2191-2195 (2006).

[142] J. M. Luther, M. C. Beard, Q. Song, M. Law, R. J. Ellingson, and A. J. Nozik, Multiple Exciton Generation in Films of Electronically Coupled PbSe Quantum Dots, Nano Lett. 7, 1779-1784 (2007).

[143] C. E. Finlayson, A. Amezcua, P. J. A. Sazio, P. S. Walker, M. C. Grossel, R. J. Curry, D. C. Smith, and J. J. Baumberg, Infrared Emitting PbSe Quantum-Dots for Telecommunications-Window Applications, J. Mod. Optics 52, 955-964 (2005).

[144] M. J. Fernée, E. Thomsen, P. Jensen, and H. Rubinsztein-Dunlop, Highly efficient luminescence from a hybrid state found in strongly quantum confined $\mathrm{PbS}$ nanocrystals, Nanotechnology 17, 956-962 (2006).

[145] V. I. Klimov, J. A. McGuire, R. D. Schaller, and V. I. Rupasov, Scaling of multiexciton lifetimes in semiconductor nanocrystals, Phys. Rev. B 77, 195324 (2008).

[146] O. V. Prezhdo, Multiple excitons and the electron phonon bottleneck in semicon- 
ductor quantum dots: An ab initio perspective, Chem. Phys. Lett. 460, 9 (2008).

[147] R. D. Schaller and V. I. Klimov, High Efficiency Carrier Multiplication in PbSe Nanocrystals: Implications for Solar Energy Conversion, Phys. Rev. Lett. 92, 186601 (2004).

[148] M. T. Trinh, A. J. Houtepen, J. M. Schins, T. Hanrath, J. Piris, W. Knulst, A. P. L. M. Goossens, and L. D. A. Siebbeles, In Spite of Recent Doubts Carrier Multiplication Does Occur in PbSe Nanocrystals, Nano Lett. 8, 1713-1718 (2008).

[149] J. A. McGuire, J. Joo, J. M. Pietryga, R. D. Schaller, and V. I. Klimov, New Aspects of Carrier Multiplication in Semiconductor Nanocrystals, Acc. Chem. Res. 41, 1810-1819 (2008).

[150] S. J. Kim, W. J. Kim, Y. Sahoo, A. N. Cartwright, and P. N. Prasad, Multiple exciton generation and electrical extraction from a PbSe quantum dot photoconductor, Appl. Phys. Lett. 92, 031107 (2008).

[151] J. R. Lakowicz, Principles of fluorescence spectroscopy, Kluwer Academic/Plenum Publishers, New York, 2nd edition, 1999.

[152] J. J. Peterson, L. Huang, C. Delerue, G. Allan, and T. D. Krauss, Uncovering Forbidden Optical Transitions in PbSe Nanocrystals, Nano Lett. 7, 3827-3831 (2007).

[153] I. Kang and F. W. Wise, Electronic structure and optical properties of $\mathrm{PbS}$ and PbSe quantum dots, J. Opt. Soc. Am. B 14, 1632-1646 (1997).

[154] P. Liljeroth, P. A. Zeijlemans van Emmichoven, S. G. Hickey, H. Weller, and B. Grandidier, Density of States Measured by Scanning-Tunneling Spectroscopy Sheds New Light on the Optical Transitions in PbSe Nanocrystals, Phys. Rev. Lett. 95, 086801 (2005).

[155] J. M. An, A. Franceschetti, S. V. Dudiy, and A. Zunger, The Peculiar electronic structure of PbSe quantum dots, Nano Lett. 6, 2728-2735 (2006).

[156] M. T. Trinh, A. J. Houtepen, J. M. Schins, J. Piris, and L. D. A. Siebbeles, Nature of the Second Optical Transition in PbSe Nanocrystals, Nano Lett. 8, 2112-2117 (2008).

[157] I. Moreels, K. Lambert, D. De Muynck, F. Vanhaecke, D. Poelman, J. C. Martins, G. Allan, and Z. Hens, Composition and Size-Dependent Extinction Coefficient of Colloidal PbSe Quantum Dots, Chem. Mater. 19, 6101-6106 (2007).

[158] D. R. Lide, CRC Handbook of Chemistry and Physics, 88th Edition (Internet Version 2008), CRC Press/Taylor and Francis, Boca Raton, FL, 2008.

[159] P. G. Kik and A. Polman, Gain limiting processes in Er-doped Si nanocrystal waveguides in $\mathrm{SiO}_{2}$, J. Appl. Phys. 91, 534-536 (2002).

[160] C. Delerue and M. Lannoo, Nanostructures - Theory and Modeling, Springer-Verlag Berlin Heidelberg, 2004.

[161] C. D. Keefe and J. L. MacDonald, Optical constant, dielectric constant and molar polarizability spectra of liquid hexane between 4000 and $400 \mathrm{~cm}^{-1}$ at $25^{\circ} \mathrm{C}$, J. Mol. Liq. 121, 121-126 (2005).

[162] M. D. Leistikow, J. Johansen, A. J. Kettelarij, P. Lodahl, and W. L. Vos, Sizedependent oscillator strength and quantum efficiency of CdSe quantum dots con- 
trolled via the local density of states, Phys. Rev. B 79, 045301 (2009).

[163] S. Schmitt-Rink, D. A. B. Miller, and D. S. Chemla, Theory of the linear and nonlinear optical properties of semiconductor microcrystallites, Phys. Rev. B 35, 8113-8125 (1987).

[164] L. E. Brus, Electron-electron and electron-hole interactions in small semiconductor crystallites: The size dependence of the lowest excited electronic state, J. Chem. Phys. 80, 4403-4409 (1984).

[165] Y. Kayanuma, Quantum-size effects of interacting electrons and holes in semiconductor microcrystals with spherical shape, Phys. Rev. B 38, 9797-9805 (1988).

[166] Y. Wang and N. Herron, Nanometer-sized semiconductor clusters: materials synthesis, quantum size effects, and photophysical properties, J. Phys. Chem. 95, 525-532 (1991).

[167] A. F. van Driel, Light Sources in Semiconductor Photonic Materials, PhD thesis, Utrecht University, available online: http://www.photonicbandgaps.com, 2006.

[168] A. L. Efros, M. Rosen, M. Kuno, M. Nirmal, D. J. Norris, and M. Bawendi, Bandedge exciton in quantum dots of semiconductors with a degenerate valence band: Dark and bright exciton states, Phys. Rev. B 54, 4843-4856 (1996).

[169] T. G. Euser, P. J. Harding, and W. L. Vos, Broadband sensitive pump-probe setup for ultrafast optical switching of photonic nanostructures and semiconductors, Rev. Sci. Instrum., submitted (2009).

[170] T. G. Euser, A. J. Molenaar, J. G. Fleming, B. Gralak, A. Polman, and W. L. Vos, All-optical octave-broad ultrafast switching of Si woodpile photonic band gap crystals, Phys. Rev. B 77, 115214 (2008).

[171] J. J. Peterson and T. D. Krauss, Fluorescence Spectroscopy of Single Lead Sulfide Quantum Dots, Nano Lett. 6, 510-514 (2006).

[172] J. M. Harbold and F. W. Wise, Photoluminescence spectroscopy of PbSe nanocrystals, Phys. Rev. B 76, 125304 (2007).

[173] A. F. van Driel, G. Allan, C. Delerue, P. Lodahl, W. L. Vos, and D. Vanmaekelbergh, Frequency-Dependent Spontaneous Emission Rate from CdSe and CdTe Nanocrystals: Influence of Dark States, Phys. Rev. Lett. 95, 236804 (2005).

[174] J. Johansen, S. Stobbe, I. S. Nikolaev, T. Lund-Hansen, P. T. Kristensen, J. M. Hvam, W. L. Vos, and P. Lodahl, Size dependence of the wavefunction of selfassembled InAs quantum dots from time-resolved optical measurements, Phys. Rev. B 77, 073303 (2008).

[175] A. Ishimaru, Wave Propagation and Scattering in Random Media, Academic, New York, 1978.

[176] M. P. van Albada and A. Lagendijk, Observation of Weak Localization of Light in a Random Medium, Phys. Rev. Lett. 55, 2692-2695 (1985).

[177] P. Sheng, Scattering and Localization of Classical Waves in Random Media, World Scientific, Singapore, 1990.

[178] A. Lagendijk, R. Vreeker, and P. de Vries, Influence of internal reflection on diffusive transport in strongly scattering media, Phys. Lett. A 136, 81-88 (1989). 
[179] J. X. Zhu, D. J. Pine, and D. A. Weitz, Internal reflection of diffusive light in random media, Phys. Rev. A 44, 3948-3959 (1991).

[180] M. U. Vera and D. J. Durian, Angular distribution of diffusely transmitted light, Phys. Rev. E 53, 3215-3224 (1996).

[181] V. N. Bogomolov, S. V. Gaponenko, I. N. Germanenko, A. M. Kapitonov, E. P. Petrov, N. V. Gaponenko, A. V. Prokofiev, A. N. Ponyavina, N. I. Silvanovich, and S. M. Samoilovich, Photonic band gap phenomenon and optical properties of artificial opals, Phys. Rev. E 55, 7619-7625 (1997).

[182] R. Rengarajan, P. Jiang, V. Colvin, and D. Mittleman, Optical properties of a photonic crystal of hollow spherical shells, Appl. Phys. Lett. 77, 3517-3519 (2000).

[183] M. Notomi, Theory of light propagation in strongly modulated photonic crystals: Refractionlike behavior in the vicinity of the photonic band gap, Phys. Rev. B 62, 10696-10705 (2000).

[184] A. Blanco, E. Chomski, S. Grabtchak, M. Ibisate, S. John, S. W. Leonard, C. Lopez, F. Meseguer, H. Miguez, J. P. Mondia, G. A. Ozin, O. Toader, and H. M. van Driel, Large-scale synthesis of a silicon photonic crystal with a complete three-dimensional bandgap near 1.5 micrometres, Nature 405, 437-440 (2000).

[185] V. N. Astratov, A. M. Adawi, S. Fricker, M. S. Skolnick, D. M. Whittaker, and P. N. Pusey, Interplay of order and disorder in the optical properties of opal photonic crystals, Phys. Rev. B 66, 165215 (2002).

[186] J. F. Galisteo-López, E. Palacios-Lidón, E. Castillo-Martínez, and C. López, Optical study of the pseudogap in thickness and orientation controlled artificial opals, Phys. Rev. B 68, 115109 (2003).

[187] A. F. Koenderink and W. L. Vos, Light exiting from real photonic band gap crystals is diffuse and strongly directional, Phys. Rev. Lett. 91, 213902-1-213902-4 (2003).

[188] T. Yamasaki and T. Tsutsui, Spontaneous emission from fluorescent molecules embedded in photonic crystals consisting of polystyrene microspheres, Appl. Phys. Lett. 72, 1957-1959 (1998).

[189] E. P. Petrov, V. N. Bogomolov, I. I. Kalosha, and S. V. Gaponenko, Spontaneous Emission of Organic Molecules Embedded in a Photonic Crystal, Phys. Rev. Lett. 81, 77-80 (1998).

[190] M. Megens, J. E. G. J. Wijnhoven, A. Lagendijk, and W. L. Vos, Light sources inside photonic crystals, J. Opt. Soc. Am. B 16, 1403-1408 (1999).

[191] H. P. Schriemer, H. M. van Driel, A. F. Koenderink, and W. L. Vos, Modified spontaneous emission spectra of laser dye in inverse opal photonic crystals, Phys. Rev. A 63, 011801 (2001).

[192] Y. Lin, J. Zhang, E. H. Sargent, and E. Kumacheva, Photonic pseudo-gap-based modification of photoluminescence from CdS nanocrystal satellites around polymer microspheres in a photonic crystal, Appl. Phys. Lett. 81, 3134-3136 (2002).

[193] L. Bechger, P. Lodahl, and W. L. Vos, Directional Fluorescence Spectra of Laser Dye in Opal and Inverse Opal Photonic Crystals, J. Phys. Chem. B 109, 9980-9988 (2005). 
[194] I. S. Nikolaev, P. Lodahl, and W. L. Vos, Quantitative analysis of directional spontaneous emission spectra from light sources in photonic crystals, Phys. Rev. A 71, 053813 (2005).

[195] M. Li, P. Zhang, J. Li, J. Zhou, A. Sinitskii, V. Abramova, S. O. Klimonsky, and Y. D. Tretyakov, Directional emission from rare earth ions in inverse photonic crystals, Appl. Phys. B 89, 251-255 (2007).

[196] H. Noh, M. Scharrer, M. A. Anderson, R. P. H. Chang, and H. Cao, Photoluminescence modification by a high-order photonic band with abnormal dispersion in $\mathrm{ZnO}$ inverse opal, Phys. Rev. B 77, 115136 (2008).

[197] A. Brzezinski, J.-T. Lee, J. D. Slinker, G. G. Malliaras, P. V. Braun, and P. Wiltzius, Enhanced emission from fcc fluorescent photonic crystals, Phys. Rev. B 77, 233106 (2008).

[198] A. F. Koenderink and W. L. Vos, Optical properties of real photonic crystals: anomalous diffuse transmission, J. Opt. Soc. Am. B 22, 1075-1084 (2005).

[199] B. C. Kaas, B. A. van Tiggelen, and A. Lagendijk, Anisotropy and Interference in Wave Transport: An Analytic Theory, Phys. Rev. Lett. 100, 123902 (2008).

[200] A. Blanco, C. López, R. Mayoral, H. Míguez, F. Meseguer, A. Mifsud, and J. Herrero, CdS photoluminescence inhibition by a photonic structure, Appl. Phys. Lett. 73, 1781 (1998).

[201] K. Yoshino, S. B. Lee, S. Tatsuhara, Y. Kawagishi, M. Ozaki, and A. A. Zakhidov, Observation of inhibited spontaneous emission and stimulated emission of rhodamine 6G in polymer replica of synthetic opal, Appl. Phys. Lett. 73, 3506-3508 (1998).

[202] S. G. Romanov, A. V. Fokin, and R. M. de La Rue, Eu ${ }^{3}$ emission in an anisotropic photonic band gap environment, Appl. Phys. Lett. 76, 1656 (2000).

[203] J. E. G. J. Wijnhoven and W. L. Vos, Preparation of photonic crystals made of air spheres in titania, Science 281, 802-804 (1998).

[204] W. L. Vos, M. Megens, C. M. van Kats, and P. Bösecke, X-ray Diffraction of Photonic Colloidal Single Crystals, Langmuir 13, 6004-6008 (1997).

[205] M. S. Thijssen, R. Sprik, J. E. G. J. Wijnhoven, M. Megens, T. Narayanan, A. Lagendijk, and W. L. Vos, Inhibited Light Propagation and Broadband Reflection in Photonic Air-Sphere Crystals, Phys. Rev. Lett. 83, 2730-2733 (1999).

[206] A. Hartsuiker and W. L. Vos, Structural Properties of Opals Grown with Vertical Controlled Drying, Langmuir 24, 4670-4675 (2008).

[207] W. L. Vos and H. M. van Driel, Higher order Bragg diffraction by strongly photonic fcc crystals: onset of a photonic bandgap, Phys. Lett. A 272, 101-106 (2000).

[208] L. Bechger, Synthesis and Flyorescence of Opal \& Air-Sphere Photonic Crystals, PhD thesis, University of Twente, available online: http://www.photonicbandgaps.com, 2003.

[209] K. H. Hellwege and A. M. Hellwege, Eigenschaften der Materie in ihren Aggregatzuständen, Part 6, Elektrische Eigenschaften I, in Landolt-Börnstein: Zahlenwerte und Funktionen aus Physik, Chemie, Astronomie, Geophysik und Technik, J. Bartels et al., Springer-Verlag, Berlin, 1959. 
[210] W. L. Vos, M. Megens, C. M. van Kats, and P. Bösecke, Transmission and diffraction by photonic colloidal crystals, J. Phys.: Condens. Matter 8, 9503-9507 (1996).

[211] M. Jones, J. Nedeljkovic, R. J. Ellingson, A. J. Nozik, and G. Rumbles, Photoenhancement of Luminescence in Colloidal CdSe Quantum Dot Solutions, J. Phys. Chem. B 41, 11346-11352 (2003).

[212] Y. Zhang, J. He, P.-N. Wang, J.-Y. Chen, Z.-J. Lu, D.-R. Lu, J. Guo, C.-C. Wang, and W.-Y. Yang, Time-dependent photoluminescence blue shift of the quantum dots in living cells: Effect of oxidation by singlet oxygen, J. Am. Chem. Soc. 128, 13396-13401 (2006).

[213] S. R. Cordero, P. J. Carson, R. A. Estabrook, G. F. Strouse, and S. K. Buratto, Photo-Activated Luminescence of CdSe Quantum Dot Monolayers, J. Phys. Chem. B 104, 12137-12142 (2000).

[214] T. Uematsu, S. Maenosono, and Y. Yamaguchi, Photoinduced fluorescence enhancement in CdSe/ZnS quantum dot monolayers: Influence of substrate, Appl. Phys. Lett. 89, 031910 (2006).

[215] M. Yu and A. van Orden, Enhanced Fluorescence Intermittency of CdSe-ZnS Quantum-Dot Clusters, Phys. Rev. Lett. 97, 237402 (2006).

[216] C. G. Christova, J. W. Stouwdam, T. J. Eijkemans, R. A. J. Janssen, and H. W. M. Salemink, Photoluminescence enhancement in thin films of PbSe nanocrystals, Appl. Phys. Lett. 93, 121906 (2008).

[217] R. Koole, M. M. van Schooneveld, J. Hilhorst, C. de Mello Donegá, D. C. 't Hart, A. van Blaaderen, D. Vanmaekelbergh, and A. Meijerink, On the incorporation mechanism of hydrophobic quantum dots in silica spheres by a reverse microemulsion method, Chem. Mater. 7, 2503-2512 (2008).

[218] M. J. Ventura and M. Gu, Engineering Spontaneous Emission in a Quantum-DotDoped Polymer Nanocomposite with Three-Dimensional Photonic Crystals, Adv. Mater. 20, 1329-1332 (2008).

[219] A. Ishimaru, Limitation on image resolution imposed by a random medium, Appl. Opt. 17, 348-352 (1978).

[220] D. J. Durian, Influence of boundary reflection and refraction on diffusive photon transport, Phys. Rev. E 50, 857-866 (1994).

[221] C. Blum, A. P. Mosk, I. S. Nikolaev, V. Subramaniam, and W. L. Vos, Color control of natural fluorescent proteins by photonic crystals, Small 4, 492-496 (2008).

[222] D. V. O'Connor and D. Phillips, Time-correlated single photon counting, Academic Press Inc., London, 1984.

[223] W. Becker, Advanced Time-Correlated Single Photon Counting Techniques, Springer-Verlag, Berlin, 2005.

[224] P. B. Coates, NOTES: The correction for photon 'pile-up' in the measurement of radiative lifetimes, J. Phys. E: Sci. Instrum. 1, 878-879 (1968).

[225] P. R. Bevington, Data reduction and error analysis for the physical sciences, McGraw-Hill, Inc., London, 1969.

[226] A. F. van Driel, I. S. Nikolaev, P. Vergeer, P. Lodahl, D. Vanmaekelbergh, and W. L. 
Vos, Statistical analysis of time-resolved emission from ensembles of semiconductor quantum dots: Interpretation of exponential decay models, Phys. Rev. B 75, 035329 (2007).

[227] R. Kohlrausch, Theorie des elektrischen Rückstandes in der Leidener Flasche, Ann. Phys. (Leipzig) 167, 179-214 (1854).

[228] W. H. Press, S. A. Teukolsky, W. T. Vetterling, and B. P. Flannery, Numerical Recipes in Fortran, Cambridge University Press, 2nd edition, 1992.

[229] M. G. Kendall and A. Stuart, The advanced theory of statistics, Charles Griffin \& Company Ltd., London, 2nd edition, 1963.

[230] K. H. Drexhage, Influence of a dielectric interface on fluorescence decay time, J. Lumin. 1-2, 693-701 (1970).

[231] R. R. Chance, A. Prock, and R. Silbey, Lifetime of an emitting molecule near a partially reflecting surface, J. Chem. Phys. 60, 2744-2748 (1974).

[232] D. Kleppner, Inhibited spontaneous emission, Phys. Rev. Lett. 47, 233-236 (1981).

[233] S. Haroche and D. Kleppner, Cavity Quantum Electrodynamics, Phys. Today 42, 24-30 (1989).

[234] V. Lousse, J.-P. Vigneron, X. Bouju, and J.-M. Vigoureux, Atomic radiation rates in photonic crystals, Phys. Rev. B 64, 201104 (2001).

[235] N. Vats, S. John, and K. Busch, Theory of fluorescence in photonic crystals, Phys. Rev. A 65, 043808 (2002).

[236] R. Wang, X.-H. Wang, B.-Y. Gu, and G.-Z. Yang, Local density of states in threedimensional photonic crystals: Calculation and enhancement effects, Phys. Rev. B 67, 155114 (2003).

[237] R. R. Chance, A. Prock, and R. Silbey, Molecular Fluorescence and Energy Transfer Near Interfaces, Adv. Chem. Phys. 37, 65 (1978).

[238] J. I. Pankove, Optical processes in semiconductors, Dover Publications, Inc., New York, 1975.

[239] R. W. Tjerkstra, F. B. Segerink, J. J. Kelly, and W. L. Vos, Fabrication of threedimensional nanostructures by focused ion beam milling, J. Vac. Sci. Technol. B 26, 973-977 (2008).

[240] J. Li, B. Jia, G. Zhou, and M. Gu, Direction-dependent spontaneous emission from near-infrared quantum dots at the angular band edges of a three-dimensional photonic crystal, Appl. Phys. Lett. 91, 254101 (2007).

[241] J. Li, B. Jia, G. Zhou, C. Bullen, J. Serbin, and M. Gu, Spectral Redistribution in Spontaneous Emission from Quantum-Dot-Infiltrated 3D Woodpile Photonic Crystals for Telecommunications, Adv. Mater. 19, 3276-3280 (2007).

[242] I. S. Nikolaev, W. L. Vos, and A. F. Koenderink, Accurate calculation of the local density of optical states in inverse-opal photonic crystals, J. Opt. Soc. Am. B 26, 987-997 (2009).

[243] P. N. Prasad, Nanophotonics, John Wiley \& Sons, Inc., Hoboken, New Jersey, 2004.

[244] K. Kounoike, M. Yamaguchi, M. Fujita, T. Asano, J. Nakanishi, and S. Noda, Inves- 
tigation of spontaneous emission from quantum dots embedded in two-dimensional photonic-crystal slab, Electron. Lett. 41, 1402-1403 (2005).

[245] Z.-Y. Li, L.-L. Lin, and Z.-Q. Zhang, Spontaneous Emission from Photonic Crystals: Full Vectorial Calculations, Phys. Rev. Lett. 84, 4341-4344 (2000).

[246] S. J. Tans, M. H. Devoret, H. Dai, A. Thess, R. E. Smalley, L. J. Geerligs, and C. Dekker, Individual single-wall carbon nanotubes as quantum wires, Nature $\mathbf{3 8 6}$, 474-477 (1997).

[247] S.-W. Hla, Scanning tunneling microscopy single atom/molecule manipulation and its application to nanoscience and technology, J. Vac. Sci. Technol. B 23, 1351-1360 (2005).

[248] F. H. L. Koppens, C. Buizert, K. J. Tielrooij, I. T. Vink, K. C. Nowack, T. Meunier, L. P. Kouwenhoven, and L. M. K. Vandersypen, Driven coherent oscillations of a single electron spin in a quantum dot, Nature 442, 766-771 (2006).

[249] C. López, Three-dimensional photonic bandgap materials: semiconductors for light, J. Opt. A: Pure Appl. Opt. 8, R1-R14 (2006).

[250] D. R. Smith, J. B. Pendry, and M. C. K. Wiltshire, Metamaterials and Negative Refractive Index, Science 305, 788-792 (2004).

[251] P. Benkart, A. Kaiser, A. Munding, M. Bschorr, H.-J. Pfleiderer, E. Kohn, A. Heittmann, H. Huebner, and U. Ramacher, 3D Chip Stack Technology Using Through-Chip Interconnects, IEEE Des. Test Comput. 22, 512-518 (2005).

[252] A. Badolato, K. Hennessy, M. Atatüre, J. Dreiser, E. Hu, P. Petroff, and A. Imamoğlu, Deterministic Coupling of Single Quantum Dots to Single Nanocavity Modes, Science 308, 1158-1161 (2005).

[253] L. A. Woldering, A. M. Otter, B. H. Husken, and W. L. Vos, Focused ion beam milling of nanocavities in single colloidal particles and self-assembled opals, Nanotechnology 17, 5717-5721 (2006).

[254] X. Jiang, Q. Li, and C. M. Soukoulis, Symmetry between absorption and amplification in disordered media, Phys. Rev. B 59, R9007-R9010 (1999).

[255] H. Míguez, C. López, F. Meseguer, V. Fornés, and A. Mifsud, Photonic crystal properties of packed submicrometric $\mathrm{SiO}_{2}$ spheres, Appl. Phys. Lett. 71, 1148-1150 (1997)

[256] N. F. van Hulst, J.-A. Veerman, M. F. García-Parajó, and L. Kuipers, Analysis of individual (macro)molecules and proteins using near-field optics, J. Chem. Phys. 112, 7799-7810 (2000).

[257] M. Orrit, Single-molecule spectroscopy: The road ahead, J. Chem. Phys. 117, 10938-10946 (2002).

[258] L. E. Reichl, A Modern Course in Statistical Physics, Edward Arnold, London, 1980.

[259] C. A. Leatherdale, W.-K. Woo, F. V. Mikulec, and M. G. Bawendi, On the Absorption Cross section of CdSe Nanocrystal Quantum Dots, J. Phys. Chem. B 106, 7619-7622 (2002). 
ISBN 978-90-365-2826-9 